\title{
27. GEOCHEMICAL, MINERALOGICAL, AND PALEONTOLOGICAL STUDIES
}

\author{
A. P. Lisitzin and Associates ${ }^{1}$
}

\section{CONTENTS}

\begin{tabular}{|c|c|}
\hline & Page \\
\hline Specific Features of Mineralogy & 831 \\
\hline Methods Used in Mineralogical Analysis & 834 \\
\hline $\begin{array}{l}\text { Methods Used in the Quantitative X-Ray Determina- } \\
\text { tion of Quartz and Carbonate Minerals }\end{array}$ & 834 \\
\hline Specific Features of Geochemistry & 835 \\
\hline Methods Used in Geochemical Determinations & 840 \\
\hline Spectral Method for Determination of Oxides & 841 \\
\hline $\begin{array}{l}\text { Determination of Chromium, Nickel, Cobalt, Lead, } \\
\text { Manganese, Titanium, Zirconium, Copper and Molyb- } \\
\text { denum By the Spectral Analyses Method }\end{array}$ & 843 \\
\hline $\begin{array}{l}\text { X-Ray Diffraction Method for the Study of Clay } \\
\text { Minerals }\end{array}$ & 843 \\
\hline Methods for the Determination of Zinc & 844 \\
\hline Methods for Determination of Copper and Nickel & 845 \\
\hline $\begin{array}{l}\text { Methods for Determination of Cadmium, Copper } \\
\text { Nickel and Zinc by Atomic Absorption }\end{array}$ & 845 \\
\hline Gamma-Ray Spectrometry & 846 \\
\hline Determination of Fluorine & 847 \\
\hline $\begin{array}{l}\text { Determination of Alkaline Elements by } \\
\text { Flame Photometry }\end{array}$ & 849 \\
\hline Grain-Size Analysis of Bottom Sediment & 851 \\
\hline Infrared Spectroscopy & 851 \\
\hline Determination of Diatoms & 861 \\
\hline
\end{tabular}

${ }^{1}$ A. P. Lisitzin, V. V. Serova, I. B. Zverinskaya, V. Lukashin, Z. N. Gorbunova, V. V. Gordeev, V. V. Zhurensko, A. M. Pchelintsev, Ju. I Belyaev, N. I. Popov, O. V. Shishkina, N. M. Morozov, A. P. Jouse, O. G. Kozlova, and V. V. Mukhina of Institute of Oceanology, Academy of Sciences, Moscow, USSR; Yu. N. Khodkevich, Institute of Medical-Biological Problems, Academy of Sciences, Moscow, USSR; and I. I. Plyusnina, Moscow State University, Moscow, USSR. 
1 Mineralogical Analysis of Fraction Greater Than $0.1 \mathrm{~mm}$

2A Mineralogical Analysis of Heavy Fraction in Size Range 0.1-0.01 mm

2B Mineralogical Analysis of Light Fraction in Size Range 0.1-0.01 mm

880

3A Mineralogical Analysis of Heavy Fraction in Size Range 0.1-0.05 mm

3B Mineralogical Analysis of Light Fraction in Size Range 0.1-0.05 mm

4A Mineralogical Analysis of Heavy Fraction in Size Range 0.05-0.01 mm

899

4B Mineralogical Analysis of Light Fraction in Size Range 0.05-0.01 mm

901

5 Quartz and Carbonate Minerals as Determined by X-Ray Analysis

903

6 X-Ray Analysis of Clay Minerals in Fraction $<0.001 \mathrm{~mm}$

909

7 Results of Partial Silicate Analysis 911

8 Results of Determination of Amorphous Silica 922

9 Results of Analysis of Carbon Dioxide and Organic Carbon

10 Results of Determination of Phosphorus 927

11 Spectrochemical Analysis of Trace Elements 928

12 Results of the Analysis of $\mathrm{Fe}_{2} \mathrm{O}_{3}, \mathrm{TiO}_{2}$, and $\mathrm{MnO}(\%)$

13 Results of the Analysis of Calcium Oxide and Magnesium Oxide

14 Alkaline Elements as Determined by Flame Photometry

15 Determination of $\mathrm{Zn}, \mathrm{Cn}, \mathrm{Ni}, \mathrm{Cd}, \mathrm{Sr}$ by Atomic Absorption

16 Neutron Activation Analysis of Cherts 956

17 Chemical Analysis of Recent Sediments 957

Chemical Composition of Basalts and
Volcanic Ash from Leg 6

19 Results of the Analysis of Fluorine 960 


\section{SPECIFIC FEATURES OF MINERALOGY}

\author{
(A. P. Lisitzin)
}

\section{Introduction}

On the basis of the mineralogy of the coarse fraction of recent sediment samples by Soviet expeditions Petelin (1965) has proposed a series of mineralogical provinces. According to this system Sites 44 to 46 belong to the central mineralogical province, and Sites 47 to 60 to the western part of the andesite zone. Small sub-provinces differing in their mineralogical associations are distinguished within these two larger provinces.

Minerals of the andesite provinces range from 1500 to 2700 kilometers out into the ocean. The complex and multiform association of minerals is composed of clastic-continental minerals (quartz, orthoclase, some plagioclase, garnet, ilmenite, epidote, etc.), as well as pyroclastic and volcanoclastic minerals (these are derivatives of andesite lava, such as, colorless and brown volcanic glass, plagioclase, orthorhombic and monoclinic pyroxene, hornblende, magnetite, etc.). A few islands in the northwestern Pacific also supply disintegration products of tholeitic basalt, olivine basalt, and picrite basalt (MacDonald, 1949).

Thus, it can be seen that the association of minerals in the andesite zone is characterized by its great complexity and variety. The peculiar feature of this zone is the abundance of pyroclastic minerals in association with typically continental minerals.

Further out into the ocean from the andesite zone the mineral associations rapidly change. Continental minerals disappear from the association with the result that pyroclastic minerals become predominant. In the pelagic part of the andesite zone the volcanogenic minerals predominate. Volcanogenic minerals are also predominant over large parts of the central province, and eolian material (particularly quartz) predominate in its arid portions. Besides, the mineral complex of the central portion of the province is characterized by a wide distribution of authigenic (diagenetic) minerals, such as, phillipsite, celestobarite, barite, ferrous and manganese hydroxide and palagonite.

Organogenic minerals include calcareous foraminiferal tests and remains of coccolithophoridae, opal radiolarian and diatom shells, teeth and bones of fishes (collophane).

Local sub-provinces are clearly distinguished in the vicinity of islands in the central Pacific. The sub-province of the Hawaiian Islands is especially pronounced and can be traced to a distance of 1000 kilometers off shore.

In a number of cases organogenic minerals suppress completely clastic minerals, and in order to study the clastic part such fractions were often treated with 5 per cent hydrochloric acid.

The change of a mineralogical suite with depth in the holes can serve as an evidence of changing conditions of sedimentation. Of especially great interest is the distribution of quartz with depth in the holes, which is an indicator of tradewind currents in the northern Pacific, volcanic glass which is indicative of volcanic activity, and authigenic minerals which reflect the conditions of sedimentation

\section{Results}

Tables 1 to 4 (at end of this chapter) list the occurrence of light and heavy minerals in the following size ranges, respectively: $>0.1,0.1$ to $0.01,0.1$ to 0.05 , and 0.05 to 0.01 millimeters.

\section{Sites 44 to 46}

Heavy minerals in recent sediments from the area of these sites include abundant ore minerals ( 5 to 20 per cent) and monoclinic pyroxene ( 1 to 10 per cent); the light minerals include phillipsite (20 to 40 per cent), small amounts of quartz (less than 1 per cent), plagioclase (less than 10 per cent) and colorless volcanic glass (1 to 10 per cent)

The mineral complex of the sandy-aleuritic fraction (see grain size section in this chapter) of Site 44 (Horizon Ridge) is characterized by a high content of carbonate (calcite) remains of foraminifera and coccolithophoridae. After the fractions were treated with hydrochloric acid, only a small number of grains of the heavy fraction remained unchanged. Many samples lack heavy mineral grains altogether. The minerals of the heavy fraction included here are orthorhombic and monoclinic pyroxene, epidote, and separate garnet grains. Quartz content after the dissolution of carbonates ranges from less than 1 per cent to 55 per cent (44.0-2-CC). Quartz is usually associated with orthoclase which indicates an eolian supply for these minerals. Plagioclase $(n=1.540-1.565)$ is also abundant, but volcanic glass is found in quantities of less than 5 per cent.

In Hole 44-3-CC large sand and gravel fraction was found. It consists mainly of chert fragments (30 to 100 per cent), and numerous diamond grains. Diamonds usually form concentrations in the finer fractions. For example, the 0.5 to 1.0 -millimeter fraction had a trace, 0.5 to 0.25 -millimeter fraction contained 0.28 per cent, and 0.1 to 0.25 -millimeter fraction had 2.44 per cent. These diamonds are undoubtedly derived from the destruction of the diamond bit when drilling chert. The same evidence is given by a rather high content of the alloy which is used for fixing diamonds in the bit face, which under the microscope looks like bronze or native copper. 
The fine sandy fraction contains appreciable amounts of pyroxene and epidote as typical clastic minerals. The composition of the sandy-aleuritic fractions from Hole 44-4-CC is also similar. The mineral complex in Hole 44 therefore did not undergo essential changes from the Cenomanian to the Recent-testimony to the stability of trade winds and currents determining the supply of minerals to this part of the ocean.

The preliminary data from Sites 45 and 46 (abyssal floor) show a typically very low content of minerals of the heavy fraction, however, the analysis showed predominance of pyroxene and, in some layers, of amphibole. The light fraction contains quartz, plagioclase (up to 12 per cent) and green-brown glass (1.564 to 1.570 ).

Thus, this group of sites is characterized by a small content of colorless volcanic glass from the Eocene to Recent, by a high content of quartz and a relatively low content of pyroxene. Abyssal red clay is characterized by the abundance of phillipsite.

\section{Mineralogy of Sediments from the Shatsky Rise Area (Sites 47 to $\mathbf{5 0}$ )}

Despite the fact that the sites of this area are close to each other, a certain difference in mineralogical composition separates Sites 47 and 48 from Sites 49 and 50. The first two sites located on the upper part of the rise (depth 2619 to 2689 meters) are characterized by a sharp predominance of carbonate (calcite) material and by a low content of the heavy fraction. Among a small number of heavy mineral grains, orthorhombic and monoclinic pyroxene predominate, amphibole, ore minerals and garnet are found in small amounts. The predominant minerals of the light fraction are volcanic glass of varying composition, phillipsite ( 20 to 50 per cent), teeth and bones of fishes (15 to 30 per cent), quartz ( 1 to 15 per cent); all the determinations were made for the material after digestion in hydrochloric acid. As a whole, the cores are characterized by the predominance of the acid variety of volcanic glass (R.I. $=1.505$ to 1.510 ), and some interlayers contain small amounts of brown glass with a refraction index of 1.522 to 1.531. Plagioclase is found in small amounts, its refraction index being 1.56 to 1.58 .

Site 47 is characterized by a high content of glass with a refraction index of 1.525 to 1.528 in the upper cores.

The sandy fraction has been studied from Hole 47.2-4CC. It is composed mainly of the remains of carbonate and siliceous organisms, with a few grains of diamond, limonite, and rust (from drill pipe). A similar composition of this fraction was found at Hole 48.2-2-1, 145150 centimeters.

The cores from Sites 49 and 50 were taken from 4282 to 4487 meters depths, that is, in the vicinity of the critical depth for calcium carbonate $\left(\mathrm{CaCO}_{3}\right)$. Therefore, the mineral complex of the sandy-aleuritic fractions in them is much richer.

The complex of heavy minerals is characterized here by a high content of monoclinic and orthorhombic pyroxene ( 40 to 50 per cent together), the content of monoclinic pyroxene being twice that of orthorhombic pyroxene.

The amount of pyroxene exceeds greatly that of amphibole (their content is usually below 10 per cent). Appreciable vertical changes of the content of ore minerals are observed (from 8 to 20 per cent).

Among the minerals of the light fraction, colorless volcanic glass (R.I. $=1.504$ to 1.513 ) predominates, having in some interlayers the admixtures of brown (R.I. = 1.540 ) and green (R.I. $=1.555$ ) glass. The glass content ranges from a maximum in the Pleistocene to a minimum in the Tithonian. Most of the plagioclase is characterized by a refractive index of 1.555 to 1.564 , and a small number of grains have a higher index 1.570 to 1.573. The quartz content is much lower than in Sites 44 and 45 , rarely exceeding 1 per cent. Phillipsite (to 22 per cent) is common together with the teeth and bones of fishes. The content of phillipsite and of teeth and bones of fishes is particularly high in Tithonian sediments.

The sand and gravel fraction in Hole 49.1-1-5, 60 centimeters, contains rock fragments, appreciable amounts of zeolite, and many iron-manganese micronodules. The Lower Cretaceous-Tithonian sediments from Site 50 are characterized by a high content of chert fragments, siliceous and carbonate organisms as well as limonite and rust particles. Pyroxene, hornblende, barite, mica and glauconite are found as separate grains.

\section{Mineralogy of the Abyssal Floor East of Japan (Sites 51, 52)}

Both these sites are in very deep water (5744 to 5980 meters) and penetrated abyssal red clay. The sites are characterized by a high content of pyroclastic material. The most ancient sediments obtained from the sites are Cretaceous. The period of sedimentation covered by these cores is characterized by an increase of volcanic activity from the Mesozoic era to a maximum activity, as far as can be judged from these and other sites described earlier, in late Tertiary time. Evidence of this is given, in particular, by the content of colorless acid volcanic glass; its concentration in the Tertiary sediments from Site 51 is as high as 66 per cent, and in the Mesozoic sediments it is often less than 1 per cent. It is very interesting to note that the refractive index of glass at these sites has remained constant during the last 70 million years. On the one hand, this indicates the constant character of volcanic activity in the feeding province, and on the other hand, it shows good preservation of acid glass in sediments (no hydration is observed in diagenetic transformations). 
Among minerals of the heavy fraction in Sites 51 and 52 , as also for the other sites discussed above, pyroxene usually predominates (to 70 per cent) with monoclinic pyroxene twice as abundant as orthopyroxene. Abundant ore minerals and sometimes hornblende are contained in several interlayers.

The quartz content of the light fraction is not largeless than 1 per cent; in addition to volcanic glass, with a refractive index of about 1.505 to 1.510 , there are many bones and teeth of fishes (to 51 per cent) and plagioclase. The refractive index of plagioclase ranges from 1.550 to 1.570 ; it comprises 30 to 34 per cent of the light fraction in the upper layers; a downward decrease accords with the decrease in the amount of acid glass, suggesting that the plagioclase is of volcanic origin. In Hole 52.0-10-CC the refractive index of the glass sharply increases to $1.550-1.560$.

The coarse sand and gravel fractions from these sites have been found to contain mainly rock fragments, small amounts of mica, pyroxene, garnet, glauconite, iron manganese nodules and rust.

\section{Mineralogy of the Cores from the Philippine Sea and the Western Slope of the Mariana Trench} (Sites 53, 54, 60)

The cores in this area ranged down to Lower Miocene and Oligocene age. Volcanogenic minerals are distributed here most widely. The character of glass changes sharply as compared to the earlier sites. In the Philippine Sea, light green acid glass with a refractive index higher than 1.510 (with maxima to 1.555 ) predominates over colorless glass. Colorless acid volcanic glass with a refractive index of 1.504 to 1.510 predominates in the upper horizons at Site 53. The island of Guam is probably one of the sources of the colorless and light green glass (refractive index 1.510 to 1.520 ). Site 60 in the vicinity of this island penetrated Recent to Lower Miocene sediments. Light green glass with a refractive index of 1.510 to 1.520 , and of 1.525 to 1.530 in the lowermost layers predominates among the glass in this hole. Among plagioclase (10 to 20 per cent of the light fraction), grains with a refractive index of 1.561 to 1.570 and particularly from 1.561 to 1.567 are predominant.

The mineral suite in the heavy fraction at these sites is typical for the western part of the andesite zone. Monoclinic and orthorhombic pyroxene is predominant here, and, unlike the earlier sites, in approximately equal amounts; the ratio $2: 1$ of monoclinic to orthorhombic pyroxene common throughout the andesite zone is found here only in the lower layers. Epidote, garnet and amphibole have been found in small amounts.

The content of quartz is very small, usually less than 1 per cent. The sand-gravel fractions of these cores are characterized by the predominance of rock fragments, appreciable amounts of pyroxene, hornblende, epidote, garnet and volcanic glass. The content of ore mineralsilmenite and magnetite-is very small.

\section{Mineralogy of the Caroline Ridge Sediments}

(Sites 55-59)

Three of the five sites drilled here were in shallower water, and the cores mainly comprised foraminiferalnannoplankton ooze chalk (Sites 55, 56 and 57). Site 58 located close to the critical depth for dissolution of calcite contains diatom-nannoplankton ooze. The other site (59) drilled in 5554 meters of water comprised diatom ooze and zeolitic clay.

The analyses show that the group of sites of the Caroline Ridge has its own mineral suite which is characterized by a sharp predominance of monoclinic pyroxene over orthorhombic pyroxene and amphibole in the heavy fraction. In places, orthorhombic pyroxene is absent altogether. High concentrations of ore minerals with admixtures of epidote and garnet are typical of these cores.

The distinguishing feature of the light fraction is a sharp change in the composition of the volcanic glass. Brown olive glass with a high refractive index (1.594 to 1.600$)$ is predominant in all the cores studied. Colorless and pale green volcanic glass with a low refraction index is encountered rarely. Only Core 2 of Site 59 has glass with a predominant refractive index of 1.510 to 1.520 . The light fraction contains many ash particles; the plagioclase content ranges up to 10 per cent, with a refractive index in the range 1.564 to 1.570 .

Ash interlayers consisting entirely of pyroclastic material (mainly glass) are sometimes encountered. In some cases the interlayers are correlated by tephrochronological methods. In particular, samples from Hole 58.2-1-5 and Hole 57.1-3-1 are probably of the same age because olive-green glass with a refractive index of 1.520 to 1.600 and plagioclase of similar composition are noticeably predominant in them. Dating of Hole 57.1-3-1 by microfauna has shown it to be Upper Oligocene.

\section{Conclusions}

The preliminary mineralogical study of the cores shows that the sandy-aleuritic fractions vary in their mineralogical composition. Groups of drilling sites can be combined into provinces and subprovinces based on their common mineralogical composition. The observed changes of mineralogical composition in time are especially sharp when interlayers of volcanogenic material are encountered. Many sites (particularly those in the northern portion of the ship's track) are characterized, on the contrary, by uniform mineralogical composition over long periods of time, at least 70 to 100 million 
years. Minerals may be used as indicators of the stability of the trade-wind and current system in this part of the ocean, and of the stability of the composition of volcanogenic material. The change of the composition of volcanogenic material in the western part of the andesite zone recorded in the lower layers of Tertiary time and Mesozoic period indicates, apparently, largescale changes in the history of volcanic activity of Japan and the adjacent island arcs.

\section{References}

MacDonald, G. A., 1949. Hawaiian petrographic province. Bull. Geo. Soc. Am. 60, (10).

Petelin, V.P., 1965. Specific features in the formation of the mineralogical composition of sandyaleuritic fractions in the bottom sediments of the Pacific Ocean. Lithology and Mineral Resources. N.4.

\section{METHODS USED IN MINERALOGICAL ANALYSIS}

(A. P. Lisitzin)

The mineralogical analysis was made by using optical and X-ray diffraction methods. The optical methods were applied to the studies of mineral fractions coarser than 0.1 millimeter (fine and medium sand according to the scale accepted in the USSR), 0.1 to 0.05 millimeter (course aleurite ${ }^{2}$ ). These fractions were obtained by a grain-size analysis of wet sediment. A number of investigations have shown previously that it is these two fractions which contain the most representative set of minerals. The results are given in Tables 1 and 3 at the end of this chapter.

The sand fraction ( 0.25 to 0.1 millimeters) was subjected to magnetic and then electromagnetic separation yielding three electromagnetic fractions. The separation of heavy minerals was achieved by using a liquid of 2.90 specific weight. The examination of minerals was made under a binocular microscope by the universally accepted methods (Chueva, 1950; Lozhkin, 1962). To study the insoluble remains in carbonate sediments, calcium carbonate was removed with the aid of a 5 per cent solution of hydrochloric acid.

The mineralogical analysis of the fraction from 0.1 to 0.05 millimeter was also made using gravity separation in liquid with 2.90 specific weight. Counts were made usually for 400 to 600 grains of both light and heavy fractions. Particularly great attention was paid to a division of volcanogenic minerals (volcanic glass, plagioclase, pyroxene), as well as, authigenic (diagenetic) minerals (phillipsite, iron-manganese minerals, celestobarite, barite). Quantitative determinations were also

\footnotetext{
${ }^{2}$ Aleurite - silt and coarse clay fraction (see Figure 4).
}

made on the products of glass devitrification (palagonite and palagonitized glass). Great attention was paid to the suite of terrigenous minerals (quartz, garnet, orthoclase, etc.).

In the mineralogical studies of the residues of carbonate sediments after acid treatment, it was found that the nondurable minerals, such as phillipsite and apatite, survive. In many cases the heavy mineral content in carbonate sediments amounts to only a few grains (as in Site 44) and for some samples it is absent altogether.

Most analytical determinations have been made at the Institute of Oceanology, the USSR Academy of Sciences, by engineers (Mrs. Kazakova and A. N. Rudakova) with control determinations carried out by the author. Data are presented in Tables 1 to 4 .

A partial mineralogical immersion analysis of the fraction from 0.05 to 0.01 millimeter, which includes large quantities of volcanogenic material of a long-range dispersal and the bulk of the eolian material from tropospheric and stratospheric fallout was also made and the results given in Table 4.

The mineralogic study of carbonate minerals and quartz was conducted by the X-ray methods for sediment as a whole and for its major fractions (Table 5). A description of methods used follows in the next section.

The pelitic fraction ( 0.01 to 0.001 millimeter was studied mineralogically to determine the clay mineralogy (Table 6). Methods used are described elsewhere in this chapter.

\section{References}

Lozhkin, V. V., 1962. Diagnostics of placer minerals. (Russian) Gosgeoltechizdat, Moscow.

Chueva, M. N., 1960. Mineralogical analysis of heavy and ore concentrates. (Russian) Gosgeoltechizdat, Moscow.

\section{METHODS USED IN THE QUANTITATIVE X-RAY DETERMINATION OF QUARTZ AND CARBONATE MINERALS}

$$
\text { (V. V. Serova) }
$$

Quantitative mineral determinations were made with a diffractometer using the internal standard method.

All the samples were ground by hand to a fine powder $(<1 \mu)$. Then each sample was mixed with the internal standard to obtain a homogenous mixture. The homogenization of the mixture was achieved by grinding the mixed powder with alcohol $(0.15$ gram of the sample plus 1 cubic-centimeter of alcohol). Good reproducibility was attained when settling the sample on glass 
(peak heights varied by only 2 to 3 millimeters and less). A precise change of the homogenous mixture ( 0.05 grams) as water suspensate was placed on an 18 by 18 millimeter cover glass, thereby covering the glass with a sediment layer of equal thickness.

A URS-501M diffractometer with Geiger counter was used. Optimum conditions were found when the reflection is rather intensive at the minimum content of minerals ( $<2$ per cent) and does not go off scale at the maximum content (about 100 per cent). This could be achieved by the selection of different slits between the X-ray tube and the meter and operation procedure. The slits from the tube were $1,0.5$, and 0.005 millimeters. Use was made of CuK $\alpha$-radiation with the nickel-filter. The Geiger counter used was of the MSTR-Y type, and every sixty-fourth impulse was fixed by an electronic counter. Time constant was four, voltage on the tube 25 kilowatts, strength of the current 10 milliamperes, scanning rate $1 \% \mathrm{~min}$, and the chart speed $6 \mathrm{~mm} / \mathrm{min}$.

Chemically pure calcium fluoride was used as an internal standard. This substance was selected for a standard since it gives an intense reflection at $3.15 \AA$ (in the same region as quartz and carbonates) and does not undergo any changes in the air. Calcium fluoride was added to each sample in amounts of 20 per cent of the sample weight.

The calibration curves were constructed from the known mixtures of pure minerals (quartz, calcite, aragonite and dolomite). The latter were used as a matrix. The mixtures contained from 2 to 20 per cent of each mineral.

The analysis of the samples was made in the middle of every week. The calibration curves were constructed on the basis of three repeated analyses of each sample. Each sample under study was analyzed three times. Calcite determinations were based on the 3.03 A peak, dolomite determinations on the $2.88 \AA$ peak, aragonite determinations on the $3.40 \AA$ peak, and quartz determinations on the $4.26 \AA$ and $3.33 \AA$ peaks. These were compared with the $3.15 \AA$ peak of calcium fluoride.

The sensitivity of the carbonate determination with the diffractometric method is 1 per cent for calcite, 1.5 to 2 per cent for dolomite and aragonite, and 1 per cent for quartz.

The average quadratic error of the determination is 2 to 3 per cent.

The results are given in Table 5 at the end of this chapter.

\section{SPECIFIC FEATURES OF GEOCHEMISTRY}

\author{
(A. P. Lisitzin)
}

\section{Introduction}

A variety of geochemical methods were used to determine the chemical composition of a large number of sediment samples.

The spectrochemical and flame-photometric varieties of the complete silicate analysis were widely used in these studies. These methods make possible the determination of the 10 sediment-forming elements $\left(\mathrm{SiO}_{2}, \mathrm{TiO}_{2}\right.$, $\mathrm{Al}_{2} \mathrm{O}_{3}, \mathrm{Fe}_{2} \mathrm{O}_{3}, \mathrm{MgO}, \mathrm{CaO}, \mathrm{Na}_{2} \mathrm{O}, \mathrm{K}_{2} \mathrm{O}, \mathrm{H}_{2} \mathrm{O}$ ) in bottom sediments and rock (Table 7). The content of the main biogenous components of the bottom sediment amorphous silica (Table 8), carbon dioxide, calcium carbonate and organic carbon (Table 9) and phosphorus (Table 10) has been determined by the classical methods of chemistry.

The content of terrigenous and volcanogenic material is established both from the indirect determination of the contribution of clastic material by the difference $100 \%-\left(\mathrm{CaCO}_{3}+\mathrm{SiO}_{2}\right.$ amorph $\left.+\mathrm{C}_{\text {org }}\right)$ and from the direct determination of a complete silicate analysis, as well as from the determination of the content of the element-indicators of clastic material $\left(\mathrm{Al}_{2} \mathrm{O}_{3}, \mathrm{TiO}_{2}\right.$, $\mathrm{Fn}, \mathrm{Cr}, \mathrm{Fe}, \mathrm{V}, \mathrm{Th})$. Since the percentage of these elements greatly depends on the presence of more widely distributed elements and compounds, the analysis of the ratio of hydrolyzate elements in rocks and sediment is of importance $\left(\mathrm{SiO}_{2} / \mathrm{Al}_{2} \mathrm{O}_{3} ; \mathrm{Al}_{2} \mathrm{O}_{3} / \mathrm{TiO}_{2} /\right.$ $\mathrm{Fn} ; \mathrm{TiO}_{2} / \mathrm{Cr}-$ see Table 7).

Numerous trace elements (Mo, Pb, Cu, Fn, Co, Ni, V, $\mathrm{Cr}, \mathrm{Zr}, \mathrm{Cd}, \mathrm{Sr}, \mathrm{Na}, \mathrm{K}, \mathrm{Li}, \mathrm{Rb}, \mathrm{Cs}$ ) can be used, for the most part, as geochemical indicators of the conditions of sedimentation.

The geochemical interpretation of the sediment cores was based on the chemical, spectrochemical, atomic absorption, flame-photometric and neutron activation methods, in conjunction with the X-ray diffraction method for the quantitative determination of quartz and carbonate minerals, and the microscopic study of sediment (smear slides, immersion slides, heavy concentrates). The combination of these analytical methods enable genetic interpretation to be made of the most widely distributed and important elements of marine sediments.

For instance, the total amount of silicic acid in a sample can be determined with the aid of the complete silicate analysis (the spectral method); this is the sum of amorphous silica (determined chemically by the X-ray and infra-red method with the control under a microscope), quartz silica (determined by the X-ray method), and silica as silicate and silica in volcanic glass (determined spectrally). 


$$
\begin{aligned}
\mathrm{SiO}_{2} \text { total } & =\mathrm{SiO}_{2} \text { amorph } \\
& +\mathrm{SiO}_{2} \text { silicate }
\end{aligned}
$$

The total amount of calcium is the sum of its portion associated with carbonates (determined chemically) and the portion associated with silicate material (determined spectrally).

$$
\mathrm{Ca}_{\text {total }}=\mathrm{Ca}_{\mathrm{CaU}_{3}}+\mathrm{Ca}_{\text {silicate }}
$$

A similar method was used for elucidating the genesis of magnesium. The total amount of magnesium in the sediment (determined in the complete silicate analysis) comprises both the portion associated with carbonates (determined chemically in a hydrocloric acid extraction) and that with the silicates.

$$
\mathrm{Mg}_{\text {total }}=\mathrm{Mg}_{\mathrm{CaMg}\left(\mathrm{CO}_{3}\right)_{2}}+\mathrm{Mg}_{\text {silicate }}
$$

So far, the following elements have been determined for the Leg 6 samples: $\mathrm{SiO}_{2}$ amorph, (Table 8) $\mathrm{CaCO}_{3}$ and $\mathrm{C}_{\text {org }}$, (Table 9), $\mathrm{Fe}_{2} \mathrm{O}_{3}, \mathrm{TiO}_{2}, \mathrm{MnO}$ (Table 12), $\mathrm{CaO}, \mathrm{MgO}$ (Table 13), $\mathrm{P}_{2} \mathrm{O}_{5}$ (Table 10) have been determined by the wet chemical methods; and, $\mathrm{SiO}_{2}$, $\mathrm{Al}_{2} \mathrm{O}_{3}, \mathrm{Fe}_{2} \mathrm{O}_{3}, \mathrm{MgO}, \mathrm{CaO}, \mathrm{MnO}, \mathrm{TiO}_{2}$ (Table 7), as well as $\mathrm{Mn}, \mathrm{Ti}, \mathrm{Cr}, \mathrm{V}, \mathrm{Ni}, \mathrm{Co}, \mathrm{Zr}, \mathrm{Pb}, \mathrm{Mo}, \mathrm{Ca}$ (Table 11) have been determined spectro-chemically. The content of $\mathrm{Na}, \mathrm{K}, \mathrm{Li}, \mathrm{Rb}, \mathrm{Cs}$ (Table 14) has been determined by the flame-photometric methods, and $\mathrm{Zn}$, $\mathrm{Cd}, \mathrm{Sr}, \mathrm{Cu}, \mathrm{Ni}$ (Table 15) have been analyzed with the aid of atomic absorption in the arc and flame. The content of $\mathrm{Mn}^{56}, \mathrm{Sc}^{46}, \mathrm{~W}^{187}, \mathrm{Fe}^{59}, \mathrm{Ca}^{48}, \mathrm{Sc}, \mathrm{C}_{2}, \mathrm{Sb}, \mathrm{Cu}$ and others has been determined by the neutron activation methods (Table 16).

A review of all the data pertinent to the methods used, their accuracy, sensitivity and the reproducibility of determinations follow in this chapter. A comparison of the analyses results obtained by wet chemistry and spectro-chemistry for some elements is given in Figure 6 at the end of this chapter.

A comparison of the tables shows that the analyses for most of the methods important elements (iron, manganese, titanium, calcium and nickle) were made twice by two different methods thereby minimizing the analytical errors. All the determinations were compared to the G-1 standard and others of the American Bureau of Standards and to a red clay standard presented by Oiva Joensuu (Institute of Marine Science, University of Miami, Florida).

The data given in this report should be considered as preliminary; it requries further checking, statistical, and graphical processing. However, the obtained results even in their present form can be used for a preliminary geochemical interpretation. The objectives of the geochemical studies are best served if the chemical determinations are used in comparison with the data yielded by the X-ray and optical mineralogical studies, the microscopic analysis of organic remains, and the study of the content of separate elements in mineral grains or monomineral fractions. In this way, the geochemical history of separate elements from the deepsea sediment cores can be traced with assurance.

\section{Complete Silicate Analysis}

Sedimentary rocks and their disintegration products contain mostly the ten oxides listed here: $\mathrm{SiO}_{2}, \mathrm{Al}_{2} \mathrm{O}_{3}$, $\mathrm{FeO}, \mathrm{Fe}_{2} \mathrm{O}_{3}, \mathrm{TiO}_{2}, \mathrm{MgO}, \mathrm{CaO}, \mathrm{Na}_{2} \mathrm{O}$, and $\mathrm{K}_{2} \mathrm{O}$, and $\mathrm{H}_{2} \mathrm{O}$. In addition, $\mathrm{BaO}, \mathrm{DrO}, \mathrm{MnO}, \mathrm{FnO}, \mathrm{Li}_{2} \mathrm{O}, \mathrm{CO}_{2}$ and $\mathrm{Cl}$ are present in small amounts. The content of water is usually less than 2 to 3 per cent, that is why it can be disregarded for general determinations; chlorine (Cl) and sulfate $\left(\mathrm{SO}_{3}\right)$ are usually contained in negligible amounts (less than 1 per cent). They increase in importance only in evaporites. The percentage of different oxides in rocks and sediments can be used as a basis for computations by the method of Niggly, Zavaritsky et al.

According to the method of Zavaritsky, the mathematical concept of the chemical composition of rock is given by the ratio between the following seven elements determined by this analyses: $\mathrm{Si}: \mathrm{Al}: \mathrm{Fe}: \mathrm{Mg}: \mathrm{Ca}: \mathrm{Na}: \mathrm{K}$. In this ratio, manganese which is usually present in small amounts, is combined with iron and titanium, conventionally, with silicon. The relations between cations of different elements are used to determine the relationship of femic and salic components of rocks, to elucidate the excess or deficiency of silicic acid and to find out "symptomatic" minerals, the peculiarities of feldspar, aluminosilicate, etc.

Unlike the interpretation of chemical analyses of magmatic rocks, the analysis of sedimentary rocks requires the clastic and biogenous parts of the sediment to be separated. As seen below, a considerable part, and sometimes the basic part, of calcium, magnesium and silicon in sedimentary rocks is usually associated with the biogenous process and may result in a confused interpretation. Because of this some additional analyses for the content of biogenous components in sediments have been made. They are excluded from the interpretation of the chemical composition of the initial rocks, and, conversely, can be taken into consideration when the sediment is regarded as an association of chemical elements.

Volcanic glass representing noncrystallized magmatic melt is of great importance in the composition of the sediment cores. The composition of volcanic glass and 
its physical properties change depending on the acidity of the melt. The typical chemical analysis of the recent sediment from the Pacific (Tables 17 A-D: abyssal red clay, radiolarian, diatom and foraminiferal oozes, respectively), sedimentary rocks and basalts (Table 18) are given; and, data on the chemical composition of the cores appears in Tables 7 to 16 .

\section{Geochemistry of Biogenous Material from the Cores}

The most important biogenous components of bottom sediments and sedimentary rocks include siliceous and carbonate material, organic carbon, part of phosphorus, iron, manganese and some other elements which are essential either in the life cycle of organisms for the construction of their soft parts, or in the construction of their tests. Siliceous and carbonate remains are of paramount importance. According to the classification accepted in the USSR, biogenous sediments are those with more than 50 per cent biogenous remains, this includes siliceous and carbonate material and organic carbon. According to the classification of Olausson (1960), the lower limit for biogenous ooze is 30 per cent.

The content of biogenous material can be determined by different methods. Amorphous silica is determined by chemical methods; in a number of cases these methods are controlled by the X-ray analyses and infrared spectroscopy. A wide application of the chemical methods for the quantitative determinations of opal in bottom sediments together with the microscopic and electron microscopic analyses of the samples makes possible the determination of both the genesis of siliceous material and its amount.

Carbonates were determined by several methods. First of all, the quantity of carbon dioxide was determined, as well as the $\mathrm{Ca}: \mathrm{Mg}$ ratio in hydrochloric acid extractions. These determinations together with a quantitative estimate of the content of carbonate minerals inferred from the X-ray analysis of sediment and with the microscopic study of the samples allow one to determine the genesis and to quantitatively evaluate the contribution of different organisms to the formation of the carbonate part of the sediment. Along with the quantitative determinations of calcium and magnesium, the ratio between them as well as the $\mathrm{Ca}: \mathrm{Sr}$ ratio is very important. The obtained results are presented in Tables 13 and 14.

The content of calcium carbonate in the sediment cores ranges in a very wide limit from less than 0.1 per cent to maximum values of 97 to 97.65 per cent (for carbon dioxide). According to the X-ray determinations, in a number of cases carbonate minerals compose practically 100 per cent of the sample.
As far as mineralogy is concerned, calcite is sharply dominant among the carbonates in all sediments and comprises more than 20 per cent of all the carbonates. The quantitative determinations of aragonite, high magnesium calcite, and dolomite have shown that aragonite is the more widely distributed of these minerals. At some sites $(47,50)$ its content is as high as 3 to 4 per cent of sediment, however, in such cases it is still far less important than calcite.

Dolomite is usually formed in thick sequences of carbonate sediment as a result of diagenesis or metasomatism. There is evidence of the formation of primary dolomite in carbonate sediments only a few thousand years of age. Therefore, it is quite natural to expect dolomite in the deep-sea drilling cores whose age is many tens of millions of years. On earlier cruises in the Atlantic Ocean concentrations of dolomite were found in sediments above the basalt. A thorough analysis of all the samples of Leg 6 has shown, however, that dolomite is either absent, or is found as separate grains, such as in trace amounts. This is confirmed by the chemical data on the content of magnesium in hydrochloric acid extractions from carbonate sediments. Typical dolomite $\left[\mathrm{CaMg}\left(\mathrm{CO}_{3}\right)_{2}\right]$ contains 30.4 per cent calcium oxide and 21.8 per cent magnesium. The weight ratio $\mathrm{CaO}: \mathrm{MgO}=1.39$. As a rule, magnesiumoxide content in the cores of Leg 6 ranges from a trace to 1 per cent, but one core from Site 53 gave 2.63 per cent, and from Site 60 another sample gave 1 to 2 per cent.

All this shows that calcite predominates among carbonates in the cores of Leg 6 . It is responsible for the composition of the tests of foraminifera and coccolithophoridae which are the major organisms forming carbonate sediments. No geochemical indicators have so far been able to determine the contribution of each group of organisms to sedimentation. This can, however, be easily deduced from a microscopic study of the sediment. The microscopic study of the cores shows that radiolarians are of the greatest importance among siliceous organisms. Radiolarians are often found in microsections of chert. They were also encountered in considerable amounts in sediments from Sites 46 and 58.

Diatoms, particularly the equatorial diatom Ethmodiscus rex, is of significance in the formation of some sediment horizons at Sites 50, 51, 58 and 59.

The chemical analysis in Table 8 shows that the content of amorphous silica in the core from Site 46 is 20.64 per cent, in separate layers from Site 52.0, up to 20.5 or 21.12 per cent, and from Site 59.2, up to 11.26 per cent. An examination of siliceous remains under the microscope indicates a higher content of organic opal. This can be attributed to the fact that skeletons of radiolarians and diatoms have delicate perforated walls and 
the real silica contribution by weight to sediments is far less than it seems. In carbonate sediments amorphous silica is usually absent or is encountered in quantities of 1 to 2 per cent. The same values have been obtained for zeolitic red clay. Interlayers enriched in volcanic glass do not reveal, as a rule, a rise in the content of authigenic opal; in other words, the glass proves to be rather stable to the natron leaching. In particular, this is clearly demonstrated by Site 60 where in places 50 to 100 per cent of the sediment is composed of volcanic glass and ash, but the amorphous silica only ranges from 1 to 2 per cent and is due to the presence of scattered radiolarians.

Typical siliceous sediments containing more than 50 per cent amorphous silica have not been discovered by drilling on Leg 6. It should be noted that some samples of diatom oozes from Antarctica contain up to 72 per cent amorphous silica. In the equatorial Pacific the amorphous silica content of the surface sediments is usually 10 to 30 per cent, rarely 30 to 50 per cent and more than 50 per cent only in radiolarian oozes and 40 to 60 per cent for Ethmodiscus diatom oozes (Lisitzin, 1966).

Organic carbon $\left(\mathrm{C}_{\text {org }}\right)$ is one of the most reliable indicators of organic matter. The study of organic carbon in the surface layer of the bottom sediment of the Pacific (Bogdanov, Lisitzin, Romankevich-in press) shows that organic carbon concentrations in the pelagic sediments determined by the Knopp-Frezenius method of wet combustion are less than 0.25 per cent. The analyses of organic-carbon distribution in the cores show that it is in the range from less than 0.05 per cent to the maximum values of 0.35 per cent recorded in the sediments from Horizon Ridge (Site 44). Most organic carbon concentrations are less than 0.25 per cent, that is, they are in the same range as those in the surface layer of the pelagic sediments. No regular decrease of organic-carbon content of the cores with age has been found in sediments up to 100 million years old. This is evidently related to the fact that the most stable part of organic carbon is preserved in the deep-sea sediments, there being no essential decay of this organic matter with considerable periods of time. This fact is also confirmed by the observations of the oxidation-reduction potential (Eh) of sediments as well as by the absence of a reduced zone in them. This is one of the fundamental differences of oceanic sediments from those of peripheral parts of the oceans and seas where a complex diagenetic reconstruction of sediment, the migration of numerous elements, and the formation of typical authigenic minerals proceed under the action of organic matter decay.

Phosphorus is also associated, to a great extent, with biogenous sedimentation. Part of it is related to the fragments of rocks and ash particles (basic rocks are especially enriched in phosphorus), separate minerals (particularly apatite) and adsorption on the surface of clay particles. In the pelagic sediments, the geochemistry of phosphorus is much dependent on the concentrations of teeth and bones of fishes which are a ubiquitous component of abyssal red clay. The phosphate matter of bones and teeth is called collophane. Phosphorus is a good indicator for the quantitative estimate of the content of teeth and bones in the cores. The phosphorus content (in terms of $\mathrm{P}_{2} \mathrm{O}_{5}$ ) ranges from 0.02 or 0.03 per cent to maximum values of 1.05 and even 1.69 per cent in the lower layers of Hole 59.2. A great amount of bones and teeth of fishes has been found here in Eocene and Paleocene zeolitic red clay and radiolarian ooze. High concentrations of $\mathrm{P}_{2} \mathrm{O}_{5}$ have been found also in some horizons at Sites 51 and 52 , however, they do not exceed 0.40 to 0.55 per cent here. Sediments of Site 51 are deposited in deep water below the critical depth of carbonate accumulation (5980 meters), they are zeolitic clay and radiolarian ooze with an appreciable amount of teeth and bones. Similar sediments have been obtained from the cores of Site 52 (5744 meters depth). No regular decrease in the phosphorus content from Recent to Upper Cretaceous has been found. This can evidently be related to a high stability of fish remains in pelagic sediments.

\section{Geochemistry of Clastic Material from the Cores}

Clastic and clay material form a considerable, and often basic part of many cores. Most clay minerals in recent sediments can be classified as terrigenous minerals (Rateev, Gorbunova, Lisitzin, Nosov, 1969). The laws governing the distribution of these minerals correspond to the laws of the distribution of other fine elastic minerals. Some of the clay minerals in the cores, such as montmorillonite, can undoubtedly be related to a volcanic origin. Thus, at the present time it is often very difficult to distinguish terrigenous fine dispersed minerals from volcanogenic; similar problems are encountered in coarse sediment fractions. Old volcanogenic strata are of great importance in the erosion of a water discharge area and islands in the western peripheral region of the Pacific. At the same time, the supply of undoubtedly pyroclastic material to sediments can often be noted. A thorough mineralogical and X-ray study of the cores often makes it possible to determine the genesis of clastic material, that is, to separate volcanogenic from terrigenous material. In many cases this study becomes easier due to the use of the indicatorelements of clastic material.

According to Goldschmidt's ideas, based on the ion potential (the ratio of ion charges $\mathrm{Z}$ to their radia $\mathrm{z}$ ), all elements can be divided into three groups by their properties: cations, hydrolytic elements, and elements of the dissolved anion complex. The ion potential for the most widely distributed elements of hydrolysis is 
in the range 3.0 to 9.5 . These are amphoteric elements forming acid and basic oxides, their ions are associated with water ions, that is, they are hydrolyzed. The elements of hydrolysis do not usually migrate in the process of diagenetic transformations of sediment. Among them there are sediment-forming elements contained in appreciable amounts $\left(\mathrm{Al}, \mathrm{Ti}, \mathrm{Fe}^{\prime \prime \prime}\right)$ and peculiar accessory elements $(\mathrm{Zr}, \mathrm{Ga}, \mathrm{Th}, \mathrm{Ta}, \mathrm{Nb}, \mathrm{Hf}, \mathrm{Cr}, \mathrm{V}, \mathrm{Mo})$.

Many elements at one valence behave as hydrolyzates and at another valence, as cations (iron, manganese, etc.). Therefore, proceeding from their relation to the reliable hydrolyzates which are used as evidenceelements ( $\mathrm{Al}, \mathrm{Ti}, \mathrm{Zr}, \mathrm{Th})$, the clastic part of iron and manganese and other elements can be determined.

Since the content of hydrolyzates greatly depends on their dilution with biogenous material, in particular with calcium carbonate and amorphous silica, it is more convenient to use their ratios rather than percentage. Silica-aluminum and aluminum-titanium modules $\left(\mathrm{SiO}_{2} /\right.$ $\mathrm{Al}_{2} \mathrm{O}_{3}$ and $\mathrm{Al}_{2} \mathrm{O}_{3} / \mathrm{TiO}_{2}$ ) and other ratios are widely used in geochemistry. The ratio $\mathrm{SiO}_{2} / \mathrm{Al}_{2} \mathrm{O}_{3}$ allows one to judge the genesis of the leucocratic part of clastic material, whereas the ratios $\mathrm{Fe}_{2} \mathrm{O}_{3} / \mathrm{TiO}_{2} ; \mathrm{TiO}_{2} / \mathrm{Th}$; $\mathrm{TiO}_{2} / \mathrm{Zr} ; \mathrm{TiO}_{2} / \mathrm{Cr} ; \mathrm{TiO}_{2} / \mathrm{Ca}$ and others, of the melanocratic part.

The change of the silica-aluminum ratio in space and time is very interesting. The major carriers of aluminum in bottom sediments are mica, orthoclase and clay minerals. Abyssal red clay has a ratio ranging from 2.4 to 3.3 , more often 3.2 to 3.3 , radiolarian ooze from the southwestern North Pacific has a ratio of 2.5; foraminiferal ooze of the Pacific, 2.7 to 3.5; tholeiitic basalt of the Pacific, 3.1, with the average 3.0 for the world ocean and 3.1 for basalt from Site 57 .

When determining the silica-aluminum ratio it should be kept in mind that part of the silica may be associated not with the clastic and clay material of sediments, but with their biogenous component (radiolarian skeletons, diatom frustules and sponge spicules). This correction may be significant and, therefore, should be taken into consideration; another correction may be introduced for free quartz. In this case, the silica-aluminum ratio will be determined chiefly by the relationship of these sediment-forming elements in aluminum silicates and in volcanic glass.

A detailed study of this relationship in comparison with the data on the mineralogical composition (the X-ray and immersion methods) makes possible a correlation by clastic material and the establishment of horizons different in the composition and genesis of the clastic part of their sediment. The analysis of these relationships in depths of the cores, such as, in time, enables one to judge the changes in the supplying provinces of the past and the peculiarities of ancient volcanic activity.
Quartz determined both by chemical methods and by $\mathrm{X}$-ray and optical analyses of sediments is an important indicator of terrigenous material. Quartz is seldom encountered in the mineral complexes of islands and volcanic arcs fringing the Pacific Ocean or in pyroclastic material. The bulk of this mineral is of continental origin. The supply of large amounts of quartz to the sediments of the arid zones of the Atlantic and Pacific Oceans in the eolian way was established long ago.

The study of soils of the Hawaiian Islands has shown that quartz contained in these soils is contributed together with products of tropospheric transport from North America (Rex, 1969). Quartz in the sediments of this part of the ocean is of a similar genesis, as well as quartz from the radioactive fallout in Japan (Miyake et al., 1956).

The quantitative determination of quartz in sediments without their division into fractions was made by the X-ray diffraction method for all the samples of Leg 6 analyzed. Parallel quartz content determinations were made by chemical methods. As the studies of eolian quartz have shown, the major part of the latter forms a fraction 2 to 10 microns, that is, beyond convenient optical resolution. Part of the quartz, however, forms a coarse-aleuritic fraction ( 0.1 to 0.05 millimeter) where it can be determined by the immersion method. The quartz content of cores from the Horizon Ridge was very small because of its dilution with carbonate material. In sediments from Site 45 , quartz makes up 5 per cent of the sediment, whereas, from Site 46 it makes up 20 per cent-the twofold variations of its content in time being observed there. Carbonate sediments from Site 47 contain much quartz (to 11 per cent) in the upper layers; down the sediment core the content of quartz drops to less than 1 per cent, yet some interlayers are rich in this mineral.

The rise in the content of quartz in the cores from the western part of the ocean (Sites 49, 50, 51, 52) seems quite natural. Most cores were taken from depths exceeding the critical depth for calcite dissolution. The proximity to Asia is determined here by a high (as much as 20 to 25 per cent) content of quartz in the cores. The cores obtained in the Philippine Sea, Mariana Trench (Sites 53, 54, 60) and from the Caroline Ridge (Sites 55 through 59) usually contain less than 1 per cent quartz and only in some cases as much as 10 per cent. Low quartz concentrations in this area can be attributed to both the peculiarities of the supplying province and the location of all these sites in the equatorial humid zone where no tropospheric transport of quartz is recorded.

The analysis of the distribution of quartz in time makes possible the preliminary conclusion that the system of trade winds in the northern arid zone of the Pacific during the last 70 or 100 million years was very stable. 
There were periods when the eolian transport was more or less intense, and these periods were apparently associated with changes of humidity in the supplying provinces of the North America and Asia.

\section{Geochemistry of Trace Elements}

The geochemical history of trace elements in the deepsea drilling cores as well as in bottom sediments is extremely complicated. Part of them is related to clastic material (especially to titanium, chromium, zirconiun and vanadium), other trace elements undoubtedly take part in a biogenous cycle (iron, manganese, zinc and molybdenum).

Among rare alkaline metals, lithium is bound together with clay minerals filling their crystalline lattice. Rubidium and cesium are also related to fine-dispersed minerals but they are adsorbed mainly on their surface. The highest concentrations of natrium $(\mathrm{Na})$ are recorded in sediments rich in minerals of the feldspar group. For the same reason, the greatest amount of natrium is found usually not in the fine-dispersed but in sandyaleuritic fractions of sediment. Part of natrium is adsorbed on the surface of fine particles. The highest concentrations of lithium in the lattice of clay minerals were recorded for montmorillonite and kaolinite, minerals which are widely distributed in the cores of Leg 6. The bulk of potassium, rubidium and cesium are associated with hydromica.

During diagenesis the mineral carriers of the alkaline elements are redistributed with the supply of part of the elements to interstitial waters. The preliminary analysis of the cores shows that such changes are not usually essential. The geochemical history of iron, manganese, partially molybdenum, zinc and some other elements is no less complicated. Part of them is bound with the lattice of minerals, that is, with clastic and clay material of sediments, another part is associated with a biogenous cycle, in particular with the formation of iron-organic and other organic compounds, finally, their adsorption on the surface of clay particles is very significant. Many elements migrate in sediments during diagenesis with the resulting formation of authigenic minerals. The formation of phillipsite, iron-manganese grains and nodules, and locally of celestobarite is of particular importance. These new minerals were seen in cores from Sites 45, 46, 47, 48, $49,50,52,53,55,58$ and 59. All this shows that the correct judgment of the geochemistry of most elements requires a thorough study of their chemical forms and an analysis of the minerals.

\section{References}

Lisitzin, A. P., 1966. Main regularities in the distribution of recent siliceous sediments and their relations with climatic zonality. Geochemistry of Silica. N. M. Strachov (Ed.) Moscow (Publ. Office, Science).
Miyake, Y., Sugiura, Y, and Vatsuragi, Y., 1956. Radioactive fallout in Aschikewa Houkaido in April 1955. J. Meteor, Soc. Japan, Ser. 2, 34 (4), 226.

Olausson, E., 1960. Description of sedimentary cores from central and western Pacific with the adjacent Indonesian Region. Rep. Swedish Deep-Sea Exped. 6.

Rateev, M. A., Garbunova, Z. N., Lisitzin, A. P. and Nosov, G. L., 1969. The distribution of clay minerals in the oceans. Sedimentology. 13, 21.

Rex, R. W. et al., 1969. Eolian origin of quartz in soils of Hawaii Islands and in Pacific pelagic sediments. Science. 163, 3864 .

\section{METHODS USED IN GEOCHEMICAL DETERMINATIONS}

(A. P. Lisitzin)

Chemical determinations were made by the same methods as those widely accepted in the USSR for the analysis of marine sediments and sedimentary rocks (Strakhov, 1957).

Calcium carbonate and organic carbon determinations were made by the wet combustion method using a Knopp-Frezenius device. Simultaneously with these determinations, calcium oxide and magnesium-oxide content was controlled spectrochemically and in hydrochloric extraction. This enables one to determine calcium forms in sedimentary rock existing as carbonates and silicates.

Amorphous silica (opal) was determined twice by a chemical method in 5 per cent sodium-carbonate extraction above a boiling water bath. In addition total silica was determined spectrochemically, and quartz silica by the X-ray method in the same samples. Thus, the quantitative evaluation of the major silica varieties in sediment: quartz, silicate, opal (amorphous) became possible. Along with the chemical determinations, amorphous silica was determined by the X-ray method (Goldberg, 1958; Calvert, 1966) and microscopically in smear slides (estimation of organic opal remains). A comparison of the methods was given earlier (Lisitzin, 1966). Comparative determinations with the aid of infrared spectroscopy have commenced.

Ferrum was determined calorimetrically with sulphasalicylic acid in ammonical solution, and at high iron content the gravimetric method was used. At the same time, in all the samples ferrum was determined spectrochemically, and to some samples the neutron-activation method of analysis was applied.

Manganese was determined calorimetrically after the sample was decomposed into $\mathrm{HF}+\mathrm{H}_{2} \mathrm{SO}_{4}$ and then melted together with potassium pyrosulphate. When the manganese content was high the gravimetric method was used. At the same time, in all the samples manganese was determined spectrochemically, and in some samples, also by the neutron-activation method. 
Titanium was determined calorimetrically with hydrogen peroxide, as well as spectrochemically.

Phosphorus was also determined calorimetrically as a phosphorusmolybdenum-vanadium complex.

The samples were analyzed in the Analytical Laboratory of the Institute of Oceanology, USSR Academy of Sciences, directed by O. I. Felenskaya. The analyses were made by M. I. Gochvat, A. G. Samosudova, N. K. Voshesenskoja, M. B. Cheremchanzeva and E. M. Mochalova.

\section{References}

Calvert, S. E., 1966. The accumulation of diatomaceous silica in the sediments of the Gulf of California. Bull. Geol. Soc. of Am. 77.

Goldberg, E. D., 1958. Determination of opal in marine sediments. J. Marine Res. 17.

Lisitzin, A. P., 1966. Main regularities in the distribution of recent siliceous sediments and their relations with climatic zonality. Geochemistry of Silica. N. M. Strakhov (Ed.) Moscow (Publ. Office, Science).

Strakhov, N. M., 1957. Methods of the study of sedimentary rocks. Collection of papers, edited by Strakhov. In Moscow Gosgeoltechzolat. I, II.

\section{SPECTRAL METHOD FOR DETERMINATION OF OXIDES}

(I. B. Zverinskaya)

This method is used to determine concentrations of the following major oxides: silica, aluminum, calcium, manganese, magnesium, titanium, and iron.

\section{Preparation of Samples for Analysis}

Fifty milligrams of a ground sample are mixed with buffer in the ratio 1:20. The sample then is reground and thoroughly mixed by hand with buffer and with alcohol in a jasper mortar for 30 minutes.

The composition of the buffer solution is as follows: coal powder, strontium carbonate and cupric oxide in the ratio $39: 19: 1.5$. Previous checking of spectral purity of all the components of the buffer solution is required.

\section{Spectral Analysis}

Spectral analysis is carried out with the aid of a diffraction spectrograph DFS-8 with a three-lense system of lighting the slit through a three-stepped reducer. The DFS-8 spectrograph has a flat diffraction grating 600 hatch $/ \mathrm{mm}$. The operating range of the instrument is 2000 to $10,000 \AA$, and linear dispersion, $6 \AA / \mathrm{mm}$. The slit width of the spectrograph is 0.027 . The spectrum of each sample is photographed three times (at random) onto a spectrographic plate of Type II, with sensitivity of 15 units ASA. Spectra of standards are photographed onto each of the plates.

Rocks with a known content of rock-forming oxides (a reliable chemical analysis) are used as standards. An alternating-current arc from the DG-2 generator is used for the excitation of the spectrum.

The upper electrodes are cone-shaped. The sample prepared for the analysis is tightly packed into the electrode crater. The arc is switched on by bringing the electrodes together at a weak current, then separating them to 4 millimeters and increasing the strength of the current 17 amperes. The analytical intervening space of 4 millimeters is kept constant during combustion. The latter proceeds until the sample is completely burned (one minute).

\section{Photometering}

The obtained spectra are photometered with the aid of the MF-2 microphotometer used for measuring the densities of darkened spectrograms. The ranges of densities of darkening are from 0 to 2 . Magnification of the instrument is $30 \mathrm{X}$, and sensitivity of the photocell is no less than 30 milliamps/lumen.

Analytical lines and comparison lines are given below:

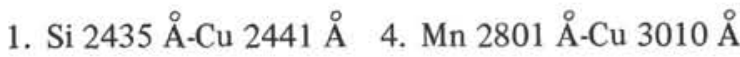

$$
\begin{aligned}
& \text { Si } 2587 \text { Å-Cu } 3010 \AA \quad \text { 5. Mg } 2775 \text { Å-Cu } 3010 \AA \\
& \text { 2. Al } 2652 \AA \text {-Cu } 3010 \AA \text { 6. Fe } 3024 \AA \text {-Cu } 3010 \AA \\
& \text { 3. Ca } 3006 \AA \text {-Si } 2531 \AA \text { 7. Ti } 2556 \AA \text { ̊-Cu } 3010 \AA
\end{aligned}
$$

Silicon-dioxide and magnesium-oxide concentrations are determined from two pairs of analytical lines.

\section{Determination of Concentrations}

Average differences of the darkening of analytical lines are calculated from three parallel spectra. Then diagrams are constructed with the following coordinates: difference of darkening $(\Delta S)$, and concentration logarithms $(\lg C)$. The diagram is used to find a logarithmic value of the concentration of the corresponding oxide after which the concentration is calculated in percentage. 
Estimate of Accuracy and Reproducibility of the Method, Limits of the Concentration to be Determined The results of analyses of G-1 standard:

\begin{tabular}{lcccc}
\hline Oxides & $\begin{array}{c}\text { Number of } \\
\text { Determinations }\end{array}$ & $\begin{array}{c}\text { Concentration } \\
(\%)\end{array}$ & $\begin{array}{c}\text { Known } \\
\text { Concentration } \\
\text { (Fleischer, 1963) } \\
(\%)\end{array}$ & $\begin{array}{c}\text { Deviation } \\
(\%)\end{array}$ \\
\hline $\mathrm{SiO}_{2}$ & 7 & 73.41 & 72.610 & 1.10 \\
$\mathrm{Al}_{2} \mathrm{O}_{3}$ & 7 & 15.00 & 14.040 & 6.80 \\
$\mathrm{CaO}$ & Not determined & & 1.390 & \\
$\mathrm{MgO}$ & Not determined & & 0.041 & \\
$\mathrm{MnO}$ & Not determined & & 0.030 & 0.51 \\
$\Sigma \mathrm{Fe}_{2} \mathrm{O}_{3}$ & 7 & 1.97 & 1.960 & \\
$\mathrm{TiO}_{2}$ & 7 & & & \\
\hline
\end{tabular}

The results of the analysis of red clay standard:

\begin{tabular}{lcccc}
\hline Oxides & $\begin{array}{c}\text { Number of } \\
\text { Determinations }\end{array}$ & $\begin{array}{c}\text { Concentration } \\
(\%)\end{array}$ & $\begin{array}{c}\text { Known } \\
\text { Concentration } \\
\text { (Jensun, personal } \\
\text { communication) } \\
(\%)\end{array}$ & $\begin{array}{c}\text { Deviation } \\
(\%)\end{array}$ \\
\hline $\mathrm{SiO}_{2}$ & 5 & 50.24 & 48.75 & 2.57 \\
$\mathrm{Al}_{2} \mathrm{O}_{3}$ & 5 & 17.18 & 17.90 & 4.03 \\
$\mathrm{CaO}$ & 5 & 2.34 & 2.06 & 13.60 \\
$\mathrm{MgO}$ & 5 & 3.58 & 3.22 & 11.20 \\
$\mathrm{MnO}$ & Not determined & & 0.58 & - \\
$\mathrm{TiO}_{2} \mathrm{O}_{3}$ & 5 & 7.40 & - & - \\
\hline
\end{tabular}

The estimate of reproducibility was made from the results of the analysis of Sample 6-45.1-1-5, $60 \mathrm{~cm}$ :

\begin{tabular}{lccc}
\hline Oxides & $\begin{array}{c}\text { Concentration } \\
(\%)\end{array}$ & $\begin{array}{c}\text { Coefficient of } \\
\text { Deviation }\end{array}$ & $\begin{array}{c}\text { Limits of the Concentrations to } \\
\text { be Determined (\%) }\end{array}$ \\
\hline $\mathrm{SiO}_{2}$ & 47.38 & 6.60 & $4.600-95.50$ \\
$\mathrm{Al}_{2} \mathrm{O}_{3}$ & 16.03 & 6.06 & $0.700-45.00$ \\
$\mathrm{CaO}$ & 3.42 & 10.10 & $0.880-60.00$ \\
$\mathrm{MgO}$ & 3.35 & 7.60 & $0.660-24.50$ \\
$\mathrm{MnO}$ & Not determined & & $0.030-0.21$ \\
$\mathrm{TiO}_{2}$ & 0.72 & 12.60 & $0.150-2.25$ \\
$\mathrm{SFe}_{2} \mathrm{O}_{3}$ & 6.10 & 6.50 & $1.900-10.00$ \\
\hline
\end{tabular}




\section{Reference}

Fleischer, W., 1963. Summary of new data on rock samples G-1 and W-1. Geochim. Cosmochim. 29, 12.

\section{DETERMINATION OF CHROMIUM, NICKEL, COBALT, LEAD, MANGANESE, TITANIUM, ZIRCONIUM, COPPER AND MOLYBDENUM BY THE SPECTRAL ANALYSIS METHOD}

$$
\text { (V. Lukashin) }
$$

\section{Preparation of the Samples}

The sample is thoroughly ground in a mortar to 200mesh after which it is mixed with a buffer in the ratio $1: 2.5$. The buffer consists of spectrally pure calcium carbonate and coal powder in the proportions 1:1.5.

\section{Standards}

Synthetic standards are used for the analysis. The percentage base components of the standards are as follows: silica - 50; calcium carbonate - 30; alumina - 8; total iron - 5 ; sodium chloride - 4 ; potassium chloride 2 ; and magnesium oxide -1 . There are seven standards with the percentage concentrations of chromium, cobalt, nickel, lead, vanadium, and molybdenum from 0.05 to 0.0005 , zirconium from 0.024 to 0.00024 , copper from 0.1 to 0.0001 , manganese from 5 to 0.15 , and titanium from 3 to 0.03 . Before combustion the standards are also mixed with a buffer.

\section{Electrodes}

Electrodes of the spectrally pure coal are used for combustion. The depth of the electrode's crater is 4 millimeters, and height, 4 millimeters. There is a channel under the crater. The upper electrode is of the same form, yet it has no crater. Thirty milligrams of the sample prepared for the analysis are placed into the electrode's crater.

\section{Excitation}

An alternating-current is produced by an arc generator DG-2 which serves as the excitation source. The strength of the current is 17 amperes and the time of the combustion of the sample is 3.5 minutes.

\section{Recording}

The DFS-8 spectrograph of $6 \AA / \mathrm{mm}$ average dispersion with the diffraction grating of $600 \mathrm{hatch} / \mathrm{mm}$ is used for the analysis. A three-sense lighting system is placed in front of the spectrograph slit. The slit of the intermediate lense is 3.2 millimeters. The spectrograph is $26 \mu$ wide. Spectra are recorded onto spectral photographic plates of Type II with sensitivity equal to 15 units according to ASA.

\section{Developing}

The plates are developed in metal hydroquinone developer at $20^{\circ} \mathrm{C}$ for 3 minutes.

\section{Photometering}

Photometering is made with the aid of the MF-2 microphotometer. The ranges of measuring darkening densities are 0 to 2 . The sensitivity of the photocell is no less than 350 milliamps/lumen.

The analytical lines are as follows:

$$
\begin{array}{ll}
\mathrm{Cr}-3014.9 \AA & \mathrm{Cu}-3274.0 \AA \\
\mathrm{Ni}-3050.8 \AA & \mathrm{Zr}-3273.05 \AA \\
\mathrm{V}-3102.3 \AA & \mathrm{Pb}-2833.1 \AA \\
\mathrm{Mo}-3194.0 \AA & \mathrm{Mn}-2534.1 \AA ; 3070.3 \AA \\
\mathrm{Co}-3453.5 \AA & \mathrm{Ti}-2841.9 \AA ; 2956.1 \AA
\end{array}
$$

The background in the vicinity of each line serves as an internal standard. The method of "three standards" is used for the determination of concentrations.

\section{Calibration Diagrams}

Calibration diagrams are constructed for each element, and for each photographic plate with the coordinates $\Delta \mathrm{s}-\lg \mathrm{C}$.

\section{Accuracy}

The accuracy of the analysis is 0.005 per cent for cobalt, nickel, vanadium, molybdenum, lead and zirconium; 0.001 per cent for chromium and copper; 0.05 per cent for manganese; and 0.03 per cent for titanium.

\section{X-RAY DIFFRACTION METHOD FOR THE STUDY OF CLAY MINERALS}

$$
\text { (Z. N. Gorbunova) }
$$

Clay minerals were studied with the $<0.001$-millimeter fraction obtained from the grain-size analysis of the samples made with sodium tripoliphosphate additions. The $<0.001$-millimeter fraction was washed twice with distilled water in a centrifuge to separate it from dissolved salts. If the samples contained carbonates, the latter were dissolved with $1 \mathrm{~N}$ hydrochloric acid and then washed out.

Amorphous iron was removed using the method of Mehra and Jackson (1960), since it creates a strong background on diffractograms and makes the identification of minerals difficult. After such treatment, the fractions were saturated with magnesium cations for which purpose the fractions were placed in a $1 \mathrm{~N}$ magnesium chloride solution for one day, after which the salt excess was washed out with distilled water in a centrifuge. The fractions were settled on 18 by 18 millimeter glass and then analyzed with the aid of a diffractometer. Each sample was analyzed three times; first, when it was saturated with magnesium cations, second, when it was saturated with glycerine, and third, when it was heated for an hour to $550^{\circ} \mathrm{C}$. 
In addition, an analysis was made on the part of the samples saturated with a lithium cation, heated to $300^{\circ} \mathrm{C}$ and saturated with glycerine, according to the method of Green-Kelly (1953). This enables montmorillonite proper to be distinguished from other minerals of this group.

In addition to this, another part of the samples was treated with $1 \mathrm{~N}$ potassium hydroxide and saturated with glycerine to determine the genesis of montmorillonite by Weaver's (1958) method. A number of samples which were supposed to contain palygorskite were heated to $340^{\circ} \mathrm{C}$ during an hour after which a displacement of the reflection was observed in the vicinity of $10 \AA$, as recommended in the paper by Heller (1960).

\section{X-Ray Performance of the Instrument}

The $<0.001$ millimeter fractions were analyzed with the aid of a diffractometer Dron-1 supplied with a scintillation counter on a copper-radiation anode having a nickel filter. The operating conditions of the diffractometer were as follows: voltage on the tube -38 kilovolts; strength of the current - 19 milliampere, slits before the sample - 1 millimeter; slits after the sample - 0.5 millimeter; window -10 volts; initial threshold - 10; and, terminal threshold - 99. The time constant is 40 ; the range of impulses per second varied depending on the intensity of reflection (200 to 2000). Scanning speed was $1^{\circ}$ per minute, chart speed 10 millimeters per minute. At such operating speed the $3.54 / 3.57$ peaks were resolved clearly enough.

The samples saturated with magnesium and glycerine were analyzed in the range from 2 to 3320 , and those heated and saturated with lithium and potassium in the range from 2 to 1320 .

\section{Identification of Minerals}

The following minerals have been identified in the samples analyzed: montmorillonite group (smectite), illite, chlorite, kaolinite groups, sometimes probably palygorskite and serpentine. In addition, mixed-layered formations have also been found. The above-mentioned groups of minerals have been identified proceeding from the universally accepted methods (Brown, 1961). The results are given in Table 6 at the end of this chapter.

\section{References}

Green-Kelly, R., 1953. Identification of montmorillonoids. Soil Sci. 4, 233.

Heller, Z., 1960. An X-ray method for the determination of small quantities of palygorskite in clay mineral mixtures. Acta Universitatis Carolinae-Geologica supplements. 1, 173.
Mehra, O. D. and Jackson, M. Z., 1960. Iron oxide removal from soils and clays by a dithionate-citrate system buffered with sodium bicarbonate. Clays and Clay Minerals. London (Pergamon Press).

The X-Ray Identification and Crystal Structures of Clay Minerals. Brown (Ed.). London, 1961.

Weaver, C. E., 1958. The effects and geological significance of potassium in fixation by expandable clay minerals derived from muscovite, biotite, chlorite and volcanic material. Am. Mineralogist. 43, (9 and $10)$.

\section{METHODS FOR THE DETERMINATION OF ZINC}

(V. V. Gordeev and Yu. N. Khodkevich)

Zinc was determined by the atomic absorption methods in solution with the aid of a two-beam one-channel device with a quartz monochromator (SFDA type). An electrodeless high-frequency lamp whose light is generated with a high-frequency $45-\mathrm{mHz}$ generator served as a translucent source. A cooled slit burner, acetylene as combustible gas, and air as oxidizer were used. A galvanometer graduated in optical density units was used as a recording device. The preparation of the samples was made in the same manner as that of alkaline elements; usually the same samples were used.

Standards with zinc concentrations of $0.1 \gamma / \mathrm{ml} ; 0.5,1$; 2; 3 and 4 were prepared from synthetic standards on a carbonate basis - calcium carbonate $\left(\mathrm{CaCO}_{3}\right)-70$ per cent, magnesium carbonate $\left(\mathrm{MgCO}_{3}\right)-12$ per cent, silica $\left(\mathrm{SiO}_{2}\right)$ - 15 per cent, iron $\left(\mathrm{Fe}_{2} \mathrm{O}_{3}\right)-1$ per cent, alumina $\left(\mathrm{Al}_{2} \mathrm{O}_{3}\right)-2$ per cent - by their acidic decomposition similar to the decomposition of the samples.

Samples with zinc concentrations higher than $4 \gamma / \mathrm{ml}$ were diluted with distilled water.

Sensitivity of the technique is $0.05 \gamma / \mathrm{ml}$ for 1 per cent absorbance. Variation coefficient is 5 per cent. The analysis of the international standard of G-1 gives a zinc concentration of $40 \mathrm{ppm}$ (average from the results of many authors is $45 \mathrm{ppm}$; See Fleischer, 1963).

Results are given in Table 15.

\section{Reference}

Fleischer, W., 1963. Summary of new data on rock samples G-1 and W-1. Geochim. Cosmochim. Acta. $29,12$. 


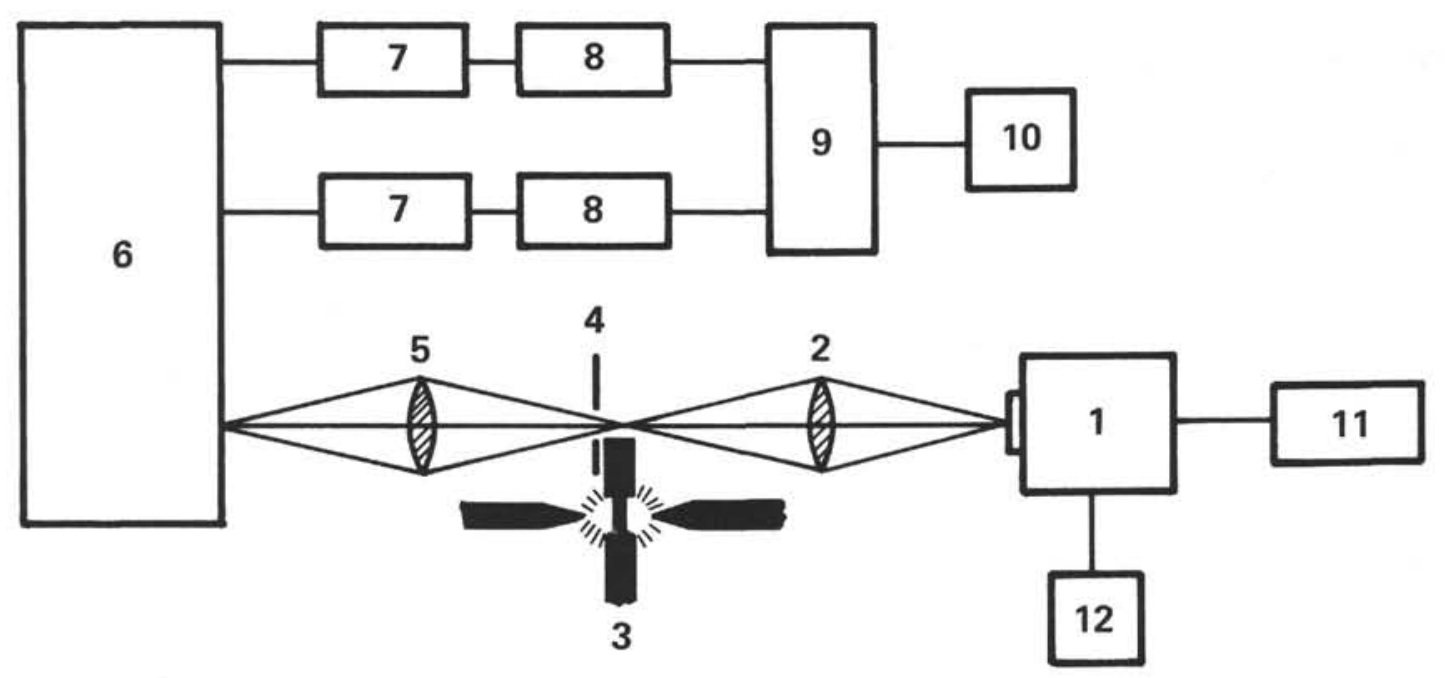

Figure 1. Schematic of the atomic absorption system with arc.

\section{METHODS FOR DETERMINATION OF COPPER AND NICKEL}

(V. V. Gordeev and V. N. Zhurenko)

Copper and nickel were determined by the atomic absorption technique in solutions with the aid of the Japanese Janagimoto spectrophotometer, model AA-1E, assembled as the American atomic absorption spectrophotometer manufactured by "Jurrel Ash" Company. The instrument is based on a single-beam circuit with a three-way passage of the beam through flame. The monochromator has a diffraction grid hollow-cathode lamp. An air/acetylene flame was used. The signal is recorded with a tenfold scale expansion recorder.

Standards and samples were prepared in the same manner as those for the zinc analysis. Copper and nickel concentrations of the standards were $0.1 ; 0.5 ; 1$; $2 ; 2$ and $4 \gamma / \mathrm{ml}$. If necessary, the samples were diluted with distilled water. Sensitivity of the technique applied was $0.05 \gamma / \mathrm{ml}$ for copper and $0.1 \gamma / \mathrm{ml}$ for nickel at 1 per cent absorption. Variation coefficient is 8 per cent for copper and 9 per cent for nickel.

The analysis of the international standard of G-1 has given $74 \mathrm{ppm}$ copper (Fleischer recommends $13 \mathrm{ppm}$ ) and less than $10 \mathrm{ppm}$ nickel (1 to $2 \mathrm{ppm}$, according to Fleischer).

\section{METHODS FOR DETERMINATION OF CADMIUM, COPPER, NICKEL AND ZINC BY ATOMIC ABSORPTION}

(V. V. Gordeev, A. M. Pchelintsev and Yu. I. Belyaev)

The identification of cadmium was made by the atomic absorption method with impulsive fractional atomization of the solid samples.
The block diagram of the device is shown in Figure 1. The radiation of the high-frequency cadmium-lamp (1) on having been modulated electrically with the aid of an acoustic generator $(410 \mathrm{c} / \mathrm{s}$ frequency) passes through the vapor of the arc impulsive atomizer (3) and is focused by lenses ( 2 and 5) onto the entrance slit of a polychromator (6) - (filter quartz polychromator of a vacuum quantometer), the outlet slits of which separate the resonance $(2288 \AA)$ and nonresonance $(2265 \AA)$ lines of cadmium. Signals from two photomultipliers (7) after cathode followers (8) arrive at the entrance of a logarithmic amplifier (9) and the logarithmic difference between the intensities of the resonance and nonresonance lines is passed to the exit of the recording potentiometer (10).

Cadmium vapor obtained with the impulsive fractional evaporation of the sample from the electrode (3) heated by a direct current arc absorbs radiation with wave length $\mathrm{Cd}-2288 \AA$, whereas radiation with wavelength Cd-2265 $\AA$ is not absorbed. Therefore, signals are proportional to optical density on the exit of the logarithmic amplifier.

The calibration of the unit was made using synthetic standards prepared on the basis of graphite powder from graphite electrodes and was checked using the international standards G-1 (cadmium content $6.10^{-6}$ per cent). Figure 2 shows a graduated diagram $(D=f$ (c)) for identifications which indicates a point corresponding to the international standard and G-1. A powdered sample weighing 10 grams was placed in a graphite electrode shaped as a wine glass and tightly pressed, evaporation being produced by a 10 -amphere direct current arc.

Sensitivity of a method with respect to thirty-six criteria is $1.10^{-6}$ per cent, and coefficient of variation 


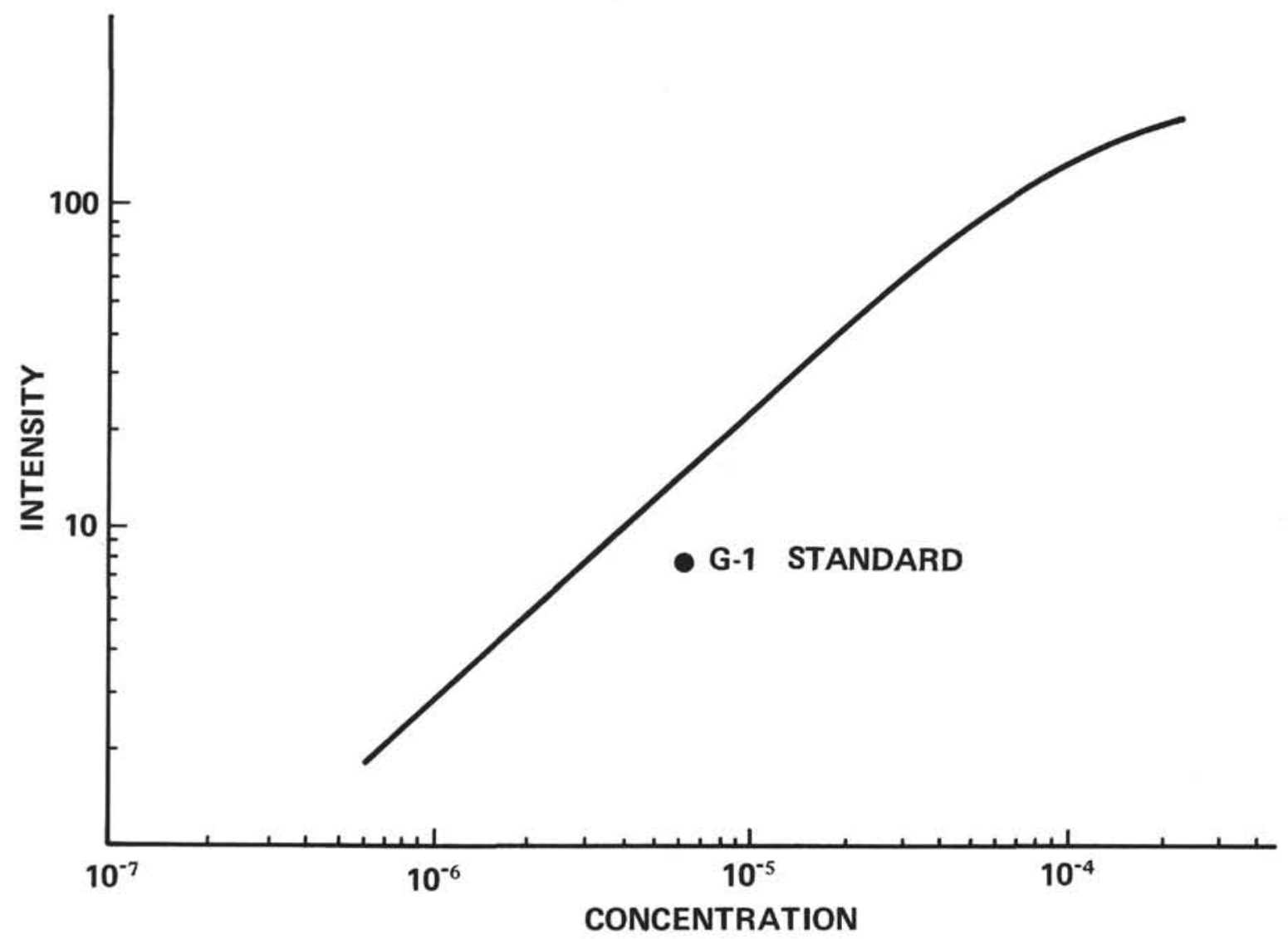

Figure 2. Calibration graph for Cadmium.

is $\sim 30$ per cent. Confidential range for concentrations from $10^{-6}$ to $10^{-5}$ with a confidential level of 0.95 is $1.5 \cdot 10^{-6}$ per cent. This level is $1.2 \cdot 10^{-5}$ for concentrations from $10^{-5}$ to $10^{-6}$

\section{GAMMA RAY SPECTROMETRY}

$$
\text { (N. I. Popov) }
$$

\section{Introduction}

The reliability of identifications of volcanic ash interlayers from the total gamma activity of the sediment core depends on the contribution of other gamma radiation sources, except for $\mathrm{K}^{40}$, to this activity. This contribution for sedimentary rocks is 50 per cent. As in oceanic sediments, the composition of the recorded gamma radiation in ash beds is little known.

Only a few measurements of this type are known, and all of them are for comparatively young formations (not older than 500,000 years), since they were made during attempts to date marine sediments by the nonsupported decay products of uranium. No data have been published so far on the content of gamma radiating radionucleides belonging to the titanium and uranium families in marine sediments of greater age.
When determining natural gamma activity of the samples aboard the ship, their total activity is determined without analyzing their energy spectrum. A detailed study of the energy spectrum is possible only in a shore laboratory.

\section{Methods}

A verification was made of the spectral composition of gamma radiation in those samples which possessed a high gamma activity as measured on the cores aboard the Challenger. The work was carried out with the aid of a gamma spectrometer consisting of a sensor (scintillation crystal $\mathrm{NaJ}(\mathrm{J} 1), 80 \times 80 \mathrm{~mm}$ ) with lead protection and a 100-channel analyzer of impulses.

Taking into account a considerable "potassium" background of the used crystal itself, due to which the spectrum region lower than approximately $1.2 \mathrm{Mev}$ proved to be an occupied band of the Compton radiation of gamma-ray quanta of $\mathrm{K}^{40}$, the measurements were made in the energy region from 1.2 to $2.8 \mathrm{Mev}$. A satisfactory resolution was observed here of photopeaks of $\mathrm{K}^{40}(1.46 \mathrm{Mev})$ and decay products of $\mathrm{U}^{238}$. $\mathrm{RaC}\left(\mathrm{Bi}^{214}\right)$ with energies 1.76 and $2.20 \mathrm{Mev}$, and $\mathrm{Th}^{232}: \mathrm{Te}^{208}$ with energy $2.62 \mathrm{Mev}$. The calibration of the instrument was made according to the marine 
sediment standard containing additional admixtures of uranium and thorium together with their decay products.

Samples for measurements represented wet natural sediment packed up into polyethylene cylinders. The weight of the standard ranged from 35 to 40 grams. Due to a relatively low rate of counting, particularly in the rigid spectrum region, each measurement lasted from 24 to 72 hours.

\section{Results}

A list of the samples studied is as follows:

1. $45.1-1-1,70$ 5. $51.1-1-4,30$ 9. $53.1-3-2,40$

2. $47.0-1-4,95$ 6. $52.0-1-2,30$ 10. 54.0-7-2, 80

3. $47.2-2-1,47$ 7. $52.0-8-3,40$ 11. $54.0-1-2,30$

4. $49.1-1-5,60$ 8. $53.0-0-2$, CC 12. $55.0-13-3,40$

Gamma spectra of each sample (the background of the instrument excluded) are presented graphically in Figure 3 . The obtained spectra clearly show that in all the samples studied, gamma radiation of $\mathrm{K}^{40}$ predominates in the total gamma activity of oceanic sediments of any age. In only one sample (47.2-2-47, 52), in addition to photopeak of $\mathrm{K}^{40}$, did the peak of the relevant decay product of uranium, $\mathrm{Bi}^{214}(1.76 \mathrm{MeV})$, became apparent. However, its contribution to the total gamma activity does not exceed, evidently, 3 per cent of the contribution of $\mathrm{K}^{40}$.

The data cited make possible a conclusion that the appreciable growth of the total gamma activity observed at some levels of the sediment column in the ocean is a unique evidence of the growth of $\mathrm{K}^{40}$ concentration at these levels.

\section{DETERMINATION OF FLUORINE}

$$
\text { (O. V. Shishkina) }
$$

\section{Introduction}

Studies of the fluorine concentration in deep sea sediments have indicated an average content of 0.054 per cent, with a range of 0.031 to 0.071 per cent. The fluorine concentrations the authors have obtained for different types of marine sediments are similar to those of Shepherd (1940) and particularly to the data of Koritnig (1951), but differ from the data of Seraphim (1951) in both their lower average values and less scatter.

A comparison of fluorine concentrations in deep sea sediments with their content of organic carbon, carbonates, phosphorus, and pelitic fraction shows no relationship between these values (Shishkina, 1966).

In the marginal parts of the ocean, on the contrary, high fluorine concentrations have been found to be confined to the sediments rich in phosphorus and organic matter.

In Shishkina (1966), the fluorine content of sediments is computed to a depth of 7 meters below the ocean floor. No variation in fluorine content with depth is observed.

This contribution contains the results of the fluorine analysis of sediment cores from Holes 51.1 and 55.0 in the Pacific Ocean.

\section{Methods}

The procedure for fluorine determinations comprises three operations: mud decomposition by fusion, fluorine distillation, and its determination proper. From 0.5 to 1 gram of fluorine are fused with six times the amount of potassium-sodium carbonate. Fluorine is distilled off in the form of fluorsilicic acid with vapour at 130 to $140^{\circ} \mathrm{C}$. In the distillate collected, fluorine is determined with an arsenazo-aluminum complex (Kuteinikov, Brodskaya, Lanskay, 1962). The optical density (measured with the aid of a light filter) has a maximum at 580 millimicrons. A detailed description of the method was given earlier (Shishkina, Pavlava, Eykova, 1967).

\section{Results}

Fluorine content of the sediment cores was determined as deep as 130 meters from the sea floor. The obtained data are presented in Table 19, where fluorine content is given as an average of two-three parallel determinations differing by no more than \pm 0.002 per cent.

Samples from Hole 51.1 are oxidized, practically carbonate-free muds with a low content of organic matter (Table 9). In four samples from Hole 51.1 the fluorine content was somewhat higher than its average concentration ( 0.054 per cent) and shows an increase with depth. The fluorine content in the sediment at the maximum depth (126.8 meters) of this site was 0.16 per cent, that is, approximately three times as great as its average. Samples from Hole 55.0 are chalk oozes with a low content of organic matter (Table 9), phosphorus (Table 10) and silicic acid (Table 8). In four samples from this hole down to 60 meters depth, the fluorine content ranged from 0.030 to 0.074 per cent, that is, within the same limits as those established earlier for the upper layers of Quaternary marine sediments (down to 7 meters). From 60 meters to 130 meters depth the fluorine concentration in these carbonate sediments was only 0.020 per cent and remained at this low level. Thus, in this case no increase of fluorine concentration (as reported by Carpenter, 1969) was observed in carbonate sediments with more than 90 per cent calcium (Table 9); on the contrary, the fluorine content was extremely low. 
$\stackrel{\infty}{\infty}$
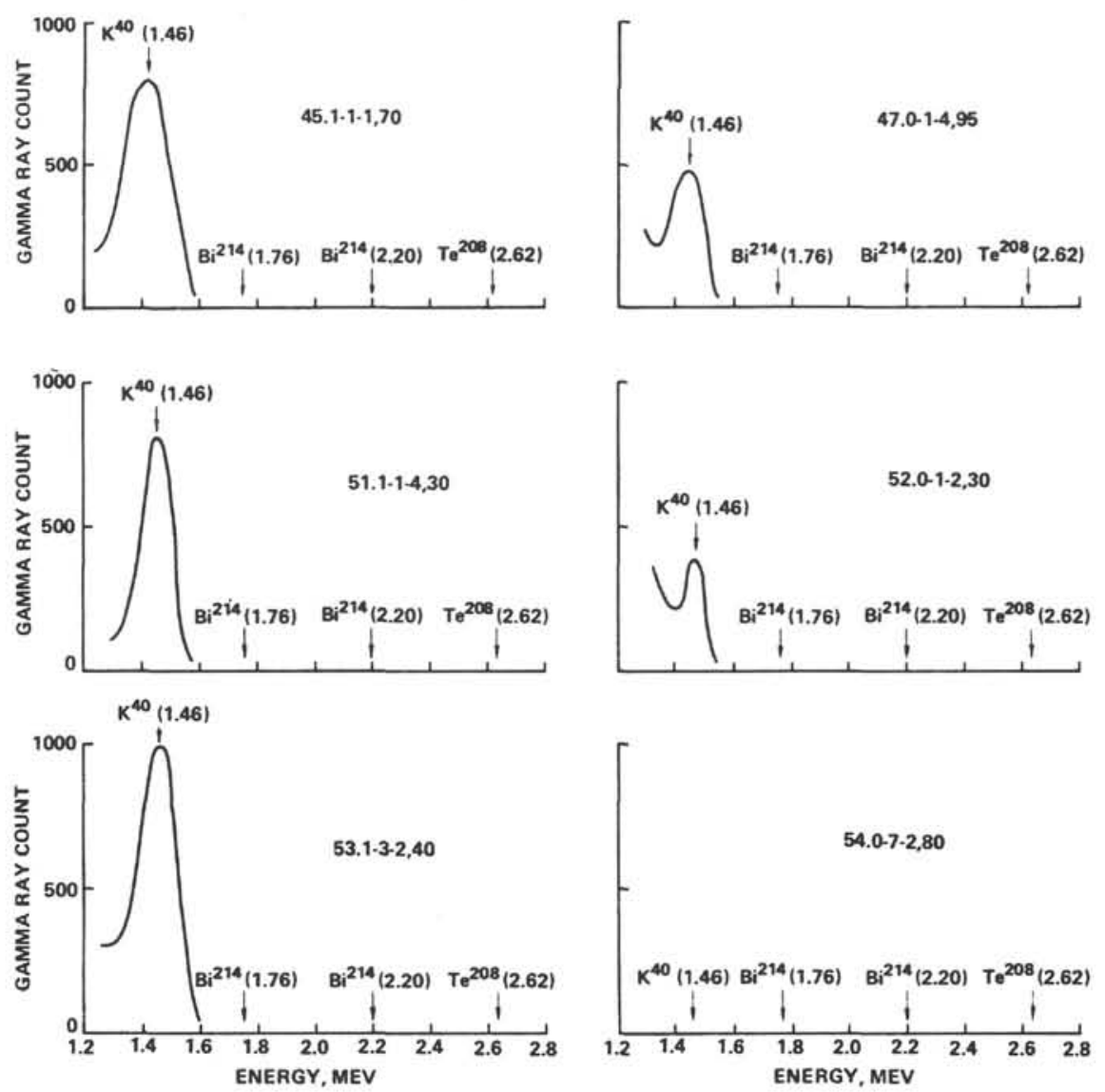
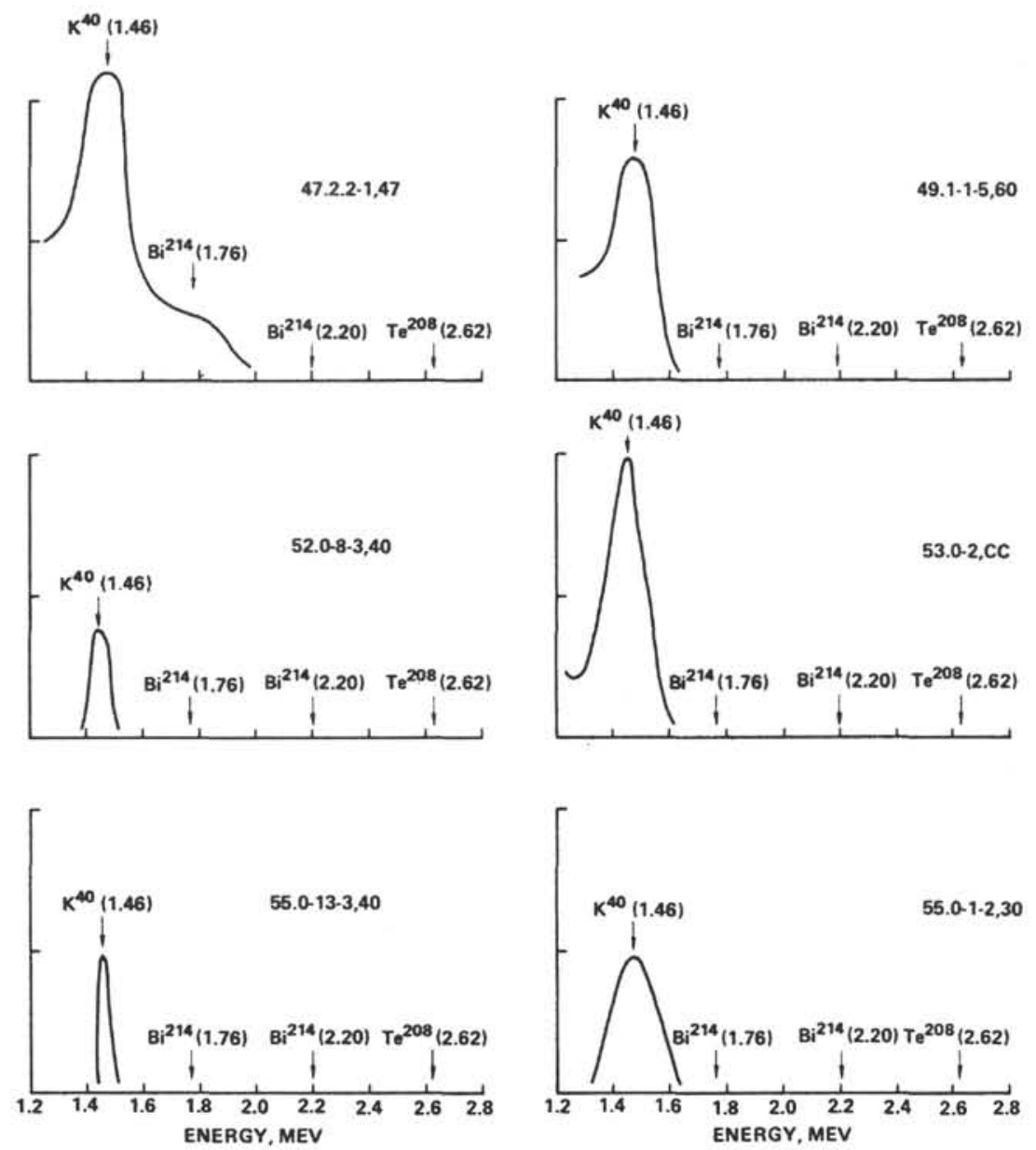

Figure 3. Gamma ray spectra of Leg 6 samples. 
As a whole, all separate data obtained so far indicate that the fluorine concentrations in sediments at depths of 100 to 130 meters below the sea floor do not differ greatly from the average content in the upper layers of Quaternary sediments. However, the fluorine concentration in the deep layers of siliceous sediments (Hole 51.1) is several times higher than its concentrations in carbonate sediments (Hole 55.0).

\section{References}

Carpenter, R., 1969. Factors controlling the marine geochemistry of fluorine. Geochim. Cosmochim. Acta. 33, 1153.

Koritnig, S., 1951. Beitrag zur Geochemie des Fluor. Geochim. Cosmochim. Acta. $12,89$.

Kuteinikov. A. F., Brodskaya, V. M. and Lanskoy, G. A., 1962. The Arsenazo-aluminium method of fluoride determination. J. Anal. Chem. 17, 1.

Seraphim, R. M., 1951. Some Aspects of the Geochemistry of Fluorine. (Thesis, Massachusetts Inst. of Technology.)

Shepherd, E. S., 1940. Notes on the fluorine content of rocks and ocean bottom samples. Am. J. Sci. 2382 , 117.

Shishkina, O. V., 1966. Fluorine in interstitial waters and marine sediments. Geochemistry. 2.

Pavlova, G. A. and Bykova, V. S., 1967. Fluorine distribution in interstitial waters and sediment of the Black Sea. Trudy Inst. Okeanolojii AN SSR. 83.

\section{DETERMINATION OF ALKALINE ELEMENTS BY FLAME PHOTOMETRY}

(N. M. Morozov)

\section{Methods}

\section{Decomposition and Dilution of the Samples}

A sample weighing 0.5 to 1 gram was placed in a glass beaker to which 3 milliliters of hydrochloric acid (1:1) were added; the sample had previously been wetted with distilled water $(\sim 1$ milliliter). After mixing the solution and adding to it 3 milliliters more of hydrochloric acid (1:1), the sample was left to stand overnight.

On the following day, another 3 milliliters of acid $(\mathrm{HCl}-1: 1)$ were added to the solution, and after evolution of carbon dioxide ceased the undecomposed residue was filtered off. The filters were first dried in porcelain crucibles in a closed electric oven (until they became black) and then burnt in muffle ovens at $t \approx$ $500^{\circ} \mathrm{C}$. After cooling, the residue was placed in platinum bowls, wetted with water ( 2 to 3 milliliters), covered with 10 to 15 milliliters of hydrogen fluoride (concentrated) and 0.4 to 0.5 milliliter of $\mathrm{HClO}_{4}(70$ per cent) and heated on a sand bath at 200 to $250^{\circ} \mathrm{C}$ to wet salts. This procedure was repeated. For a more complete removal of fluorine, 15 milliliters of distilled water and from 0.4 to 0.5 milliliter of $\mathrm{HClO}_{4}$ were added to the samples after which they were steamed dry. This was repeated three times. Filtrates (obtained after the samples were treated with hydrochloric acid) were added to the dry residue of the samples, the mixtures were boiled for about 5 minutes after which, together with the undecomposed residue, they were placed into beakers and kept there for a night.

On the following day, the samples were filtered. If the sample was carbonate-free, 5 milliliters of buffer solution of lantharum $(10,000 \gamma / \mathrm{ml})$ were added to the filtrate to eliminate the depressing effect of aluminum in strontium determinations; the solution in a measuring flask was made up to 100 milliliters.

For sodium and potassium determinations, an aliquot part of 1 milliliter volume was taken from the solution and diluted by 100 -fold (in case of carbonate-free samples) and by 50 -fold (in case of carbonate samples).

One milliliter of buffer solution potassium $(50,000$ $\gamma / \mathrm{ml}$ ) was added to the remaining part of the sample (99 milliliters); this solution was used for the flamespectrophotometric determination of lithium, rubidium, cesium, and the atomic absorption analysis for magnesium, strontium, zinc, nickel and copper.

\section{Photometering Technique}

Alkaline elements were determined with the aid of a two-channel flame-spectrophotometric device with the application of a turbulent oxy-hydrogen flame (Figure 1).

Sodium and potassium were determined by a method of comparison with the calibration diagrams after photometering the solutions of samples and standards. The standard solutions were prepared by a subsequent dilution with a distillate of the solution containing 10 $\gamma / \mathrm{ml}$ of potassium prepared, in its turn, from sodium and potassium head-solutions $(1000 \gamma / \mathrm{ml})$.

A series of the standard solutions contained the following concentrations of sodium and potassium: $0.1,0.5$, $1.0,2.5$ and $5.0 \mathrm{\gamma} / \mathrm{ml}$. In the case of carbonate samples, in addition to sodium and potassium, a series of the standards contained a constant amount of calcium $(15 \gamma / \mathrm{ml})$.

Lithium, rubidium and cesium were determined also to be the method of comparison with the calibration diagrams. The standard solutions were prepared by diluting the solution which contained $2.5 \gamma / \mathrm{ml}$ of lithium, $5 \gamma / \mathrm{ml}$ of rubidium and $1 \gamma / \mathrm{ml}$ of cesium, and was prepared from the standard head-solutions of these 
elements $(1000 \gamma / \mathrm{ml})$. A working series of the standards contained the following concentrations of the elements and buffer to be determined:

\begin{tabular}{ccccccc}
\hline $\begin{array}{c}\text { Solution } \\
\text { No. }\end{array}$ & $\mathrm{Li}$ & $\mathrm{Rb}$ & $\mathrm{Cs}$ & $\mathrm{Na}$ & $\mathrm{K}$ & $\begin{array}{c}\mathrm{HC1} \\
\text { (conc.) }\end{array}$ \\
\hline 1 & 2.5 & 5.00 & 1.00 & - & - & - \\
2 & 1.250 & 2.50 & 0.50 & 1000 & 1000 & 6.25 \\
3 & 0.500 & 1.00 & 0.20 & 1000 & 1000 & 6.25 \\
4 & 0.250 & 0.50 & 0.10 & 1000 & 1000 & 6.25 \\
5 & 0.125 & 0.25 & 0.05 & 1000 & 1000 & 6.25 \\
6 & 0.050 & 0.10 & 0.02 & 1000 & 1000 & 6.25 \\
\hline
\end{tabular}

\section{Estimate of Sensitivity, Reproducibility and Error of the Analysis}

The photometering of the samples was made in the range of the concentrations corresponding to the linear sections of the calibration diagrams or the scale limits of the instruments. These concentration ranges are taken to be values of the minimum and maximum sensitivity and are as follows:

$\begin{array}{ll}\mathrm{Na} \text { and } \mathrm{K} & 0.05-3.0 \gamma / \mathrm{ml} \\ \mathrm{Rb} & 0.05-1.6 \\ \mathrm{Li} & 0.01-0.8 \\ \mathrm{Cs} & 0.50-1.3\end{array}$

The reproducibility of the method is illustrated below. The results of a complete analysis (including decomposition) of ten parallel determinations of one carbonate sample $(55.0-13-3,40 \mathrm{~cm})$ and one red clay sample $(49.1-1-5,60 \mathrm{~cm})$ are as follows:

\begin{tabular}{c|c|c|c|c}
\hline \multirow{2}{*}{ Element } & \multicolumn{2}{|c|}{ Carbonate Sediment } & \multicolumn{2}{c}{ Red Clay } \\
\cline { 2 - 4 } & $\begin{array}{c}\text { Average of 10 } \\
\text { determinations (\%) }\end{array}$ & Vc \% & $\begin{array}{c}\text { Average of 10 } \\
\text { determinations (\%) }\end{array}$ & Vc \% \\
\hline $\mathrm{Na}$ & 0.28 & \pm 14 & 0.55 & \pm 4.2 \\
$\mathrm{~K}$ & 0.08 & \pm 36 & 1.32 & \pm 11.0 \\
$\mathrm{Li}$ & $0.24 \times 10^{-3}$ & \pm 21 & $6.0 \times 10^{-3}$ & \pm 5.7 \\
$\mathrm{Rb}$ & Trace & - & $9.2 \times 10^{-3}$ & \pm 11.5 \\
$\mathrm{Cs}$ & No & - & Trace & - \\
\hline
\end{tabular}

The results of four determinations of alkaline elements in a standard sample of red clay are as follows:

\begin{tabular}{c|c|c|c}
\hline Element & $\begin{array}{c}\text { Average of } 4 \\
\text { determinations (\%) }\end{array}$ & $\begin{array}{c}\text { Concentration according } \\
\text { to the sample's } \\
\text { passport }^{\mathrm{a}}(\%)\end{array}$ & $\begin{array}{c}\text { Deviation of the } \\
\text { average from the } \\
\text { value in the passport }\end{array}$ \\
\hline $\mathrm{Na}$ & 1.42 & 1.52 & -6.5 \\
$\mathrm{~K}$ & 2.41 & 2.91 & -17.0 \\
$\mathrm{Li}$ & $1.5 \times 10^{-3}$ & $6.6 \times 10^{-3}$ & +14.0 \\
$\mathrm{Rb}$ & $17.1 \times 10^{-3}$ & $16.0 \times 10^{-3}$ & +7.0 \\
$\mathrm{Cs}$ & $0.9 \times 10^{-3}$ & - & - \\
\hline
\end{tabular}

\footnotetext{
${ }^{\mathrm{a}}$ Red clay sample was presented by Dr. Oliva Joensuu,
} Institute of Marine Science, University of Miami. 


\section{GRAIN SIZE ANALYSIS OF BOTTOM SEDIMENT}

\author{
(A. P. Lisitzin)
}

Several hundred sediment samples were subjected to grain-size analysis by a method of combined continuous analysis widely accepted in the USSR. This method was developed at the Institute of Oceanography, the U.S.S.R. Academy of Sciences (Petelin, 1968), and at the present time it is the principle method of grain-size analysis used in that country. Only samples containing their original moisture are used for the analysis. The method involved the use of a dispergator, size control of the fractions under a microscope, and the division of fine fractions by a prepared analysis.

The analysis distinguishes fractions according to a metric scale. The relationship between this Russian classification and that of Wentworth and the Phi scale is shown in Figure 4.

The relationship between the major sediment-forming components (calcium carbonate, amorphous silica, iron, manganese and organic carbon) and their origins predetermine the material-genetic parts of the classification (Bezzukov and Lisitzin, 1960).

A wet sediment sample weighing ten to twenty grams was used in the analysis. Of this sample, two grams were taken for moisture determination. The sample is treated with a peptizer, 40 milliliters of $0.2 \mathrm{~N}$ solution of $\mathrm{Na}_{5} \mathrm{P}_{3} \mathrm{O}_{6}$ for carbonate-free and 50 milliliters for carbonate rich sediments. The suspensate is thoroughly mixed and passed through two sieves which collect the sand and coarse aleurite fractions, respectively.

The suspensate is washed with a weak jet of deionized water over a large glass cylinder. After washing thoroughly, water is added, a total of three liters to that already in the cylinder, and then left over night for temperature equalization. Next, 30 milliliter samples are withdrawn by pipette to determine the content of fractions of 0.01 to 0.005 millimeters, 0.005 to 0.001 millimeters and finer than 0.001 millimeters. Suspensates are placed into weighed sample boxes and dried in ovens. The sample bottles are reweighed to determine the weight of each fraction. Along with the fractions, 30 milliliters of deionized water is also taken as a control sample.

The suspensate that remained in the glass cylinder is separated from the pelitic fraction by washing, the sizes of particles being controlled under a microscope. Pelitic fractions (finer than 0.01 millimeter and finer than 0.001 milliliter) are collected in separate jars and settled in a centrifuge, the $\mathrm{Na}_{5} \mathrm{P}_{3} \mathrm{O}_{6}$ having been washed out with distilled water.
If the sediment contains appreciable amounts of sand and coarser material the initial weight of the sample is 100 to 150 grams and more. After the fraction finer than 0.05 millimeter is washed out the material is dried and split with the aid of sieves. When estimating the results of the analysis, a correction for salt content of the interstitial water is introduced taking into consideration the moisture determinations. The analysis yields percentage (weight) of the following sediment fractions: $>0.25$ millimeter (which may be further subdivided); 0.25 to 0.1 millimeter; 0.1 to 0.05 millimeter; 0.05 to 0.01 millimeter; 0.01 to 0.005 millimeter; 0.005 to 0.001 millimeter; $<0.001$ millimeter. The results obtained were used to construct cumulative curves (Figures 5 to 10 ) with a logarithmic abscissa and an arithmetic ordinate from which median diameter (Md) and sorting coefficient (So) have been determined using Trask's system. Some other mathematical parameters can be determined as well. The same cumulative curves can be used to convert from the decimal fraction system to the geometrical fractions or to the logarithmic Phi-system. The author has suggested a method of conversion to any scale and nomenclature based on the cumulative curve (Lisitzin, 1956).

The grain-size analyses have been made by Z. I. Vinenskaya, A. E. Rubtsova and L. I. Strelanova, of the Institute of Oceanology, the USSR Academy of Sciences.

The fractions obtained by the analyses were then used for mineralogical studies by optical methods (fractions coarser than 0.1 millimeter and from 0.1 to 0.05 millimeter) and X-ray methods (fractions finer than 0.01 millimeter and finer than 0.001 millimeter) and for the infrared and differential thermal analysis. Also, many fractions were analyzed to determine the content of some elements by spectrochemical methods, atomic absorption and neutron-activation.

\section{References}

Lisitzin, A. P., 1956. On the treatment of the results of grain size analyses of marine sediments. Trudy Inst. Okeanologii ANSSSR. 19.

Petelin, V. P., 1968. Grain Size Analysis of Marine Sediments. Moscow (Nauka).

\section{INFRARED SPECTROSCOPY}

$$
\text { (I. I. Plyusnina) }
$$

\section{Methods}

Absorption bands in the 1250 to 1100,830 to 750 and 530 to 460 centimeter $^{-1}$ ranges are typical of silica $\left(\mathrm{SiO}_{2}\right)$ modifications. In addition, each crystalline form of silica has its own specific bands. For $\gamma$ quartz these are $1167,784,695$ and 526 centimeter $^{-1}$ (especially two latter frequencies); whereas for $\gamma-$ 


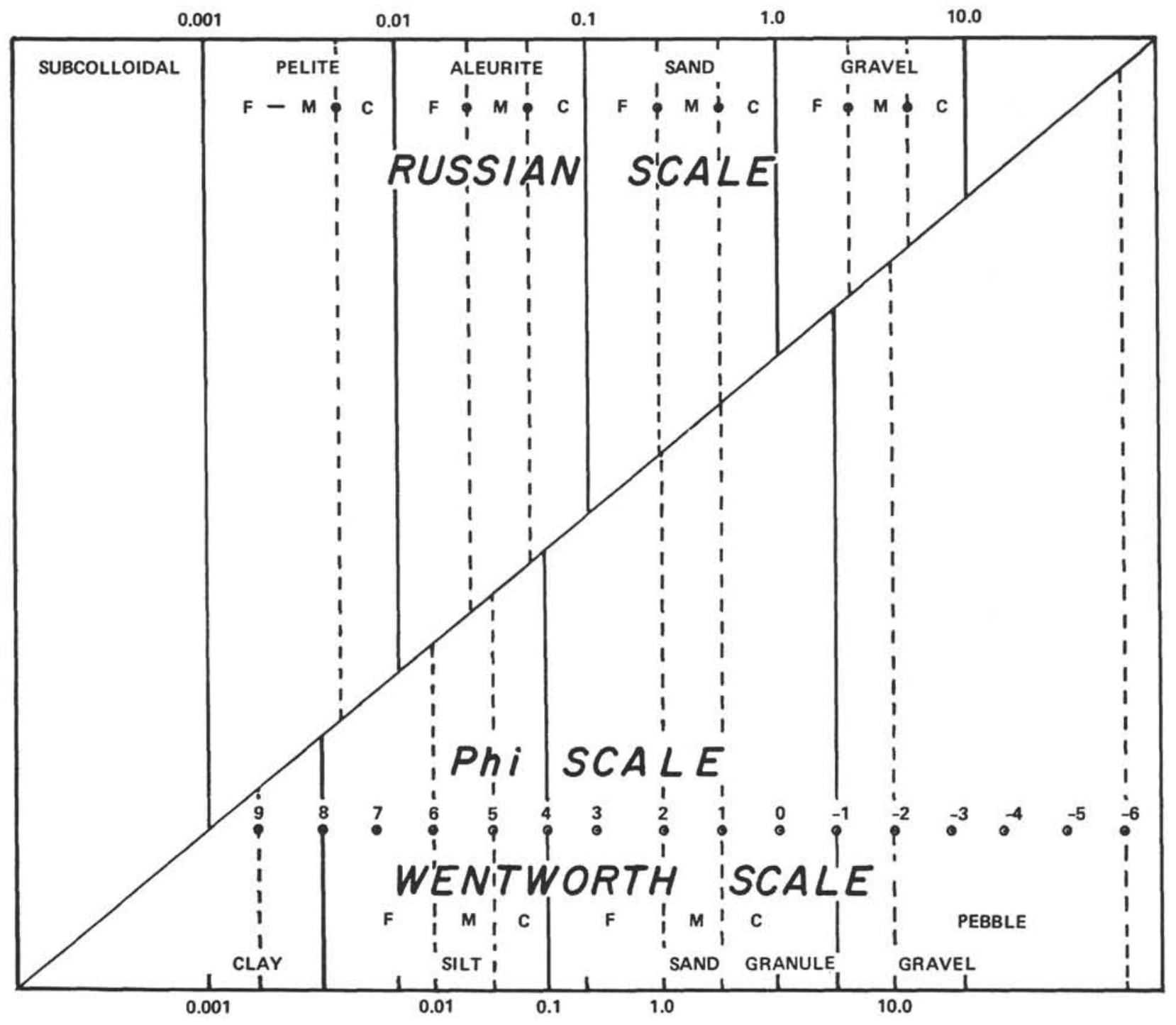

Figure 4. Grain size classification of sediments. 

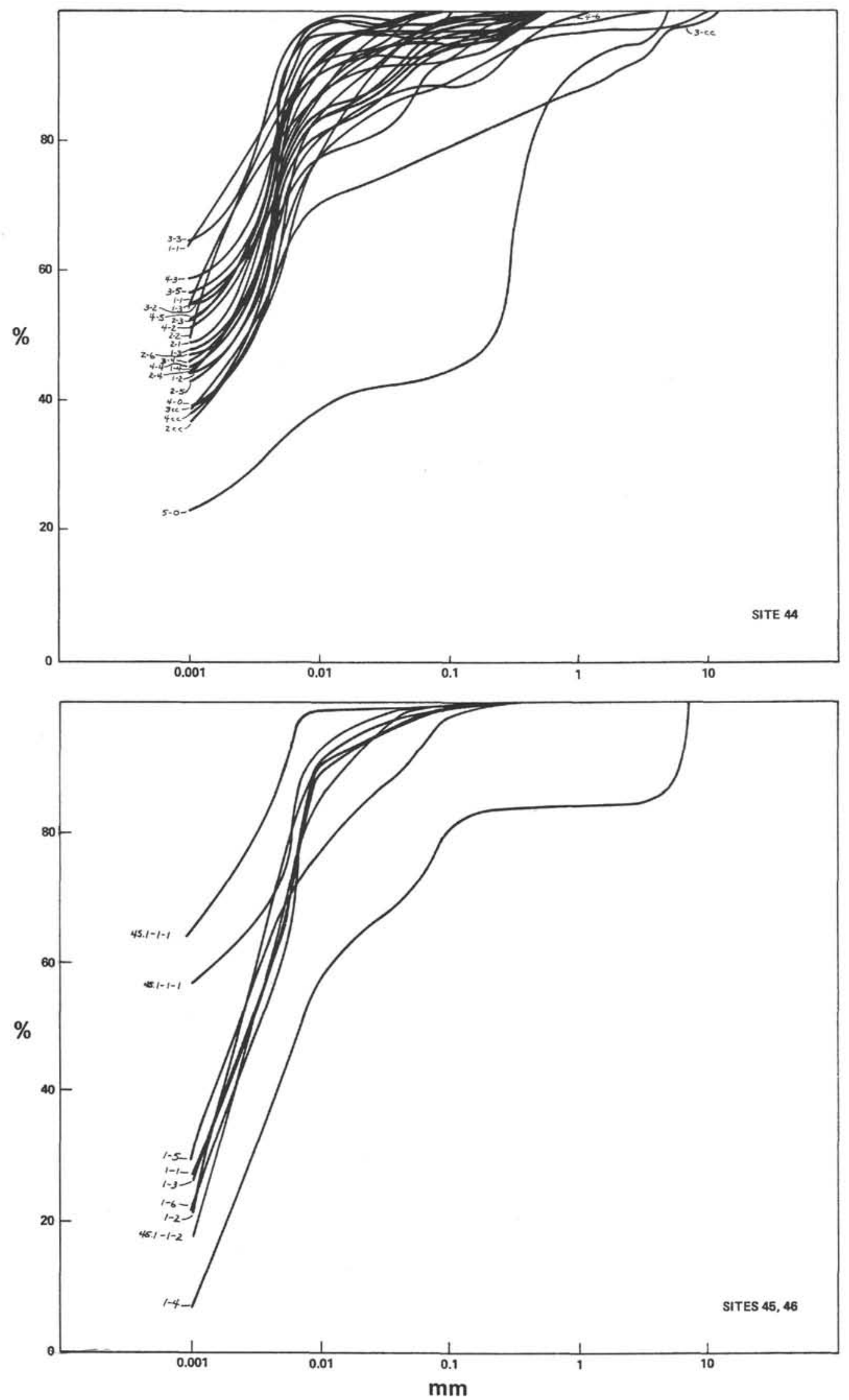

Figure 5. Cumulative curves for samples from Sites 44,45 and 46. 

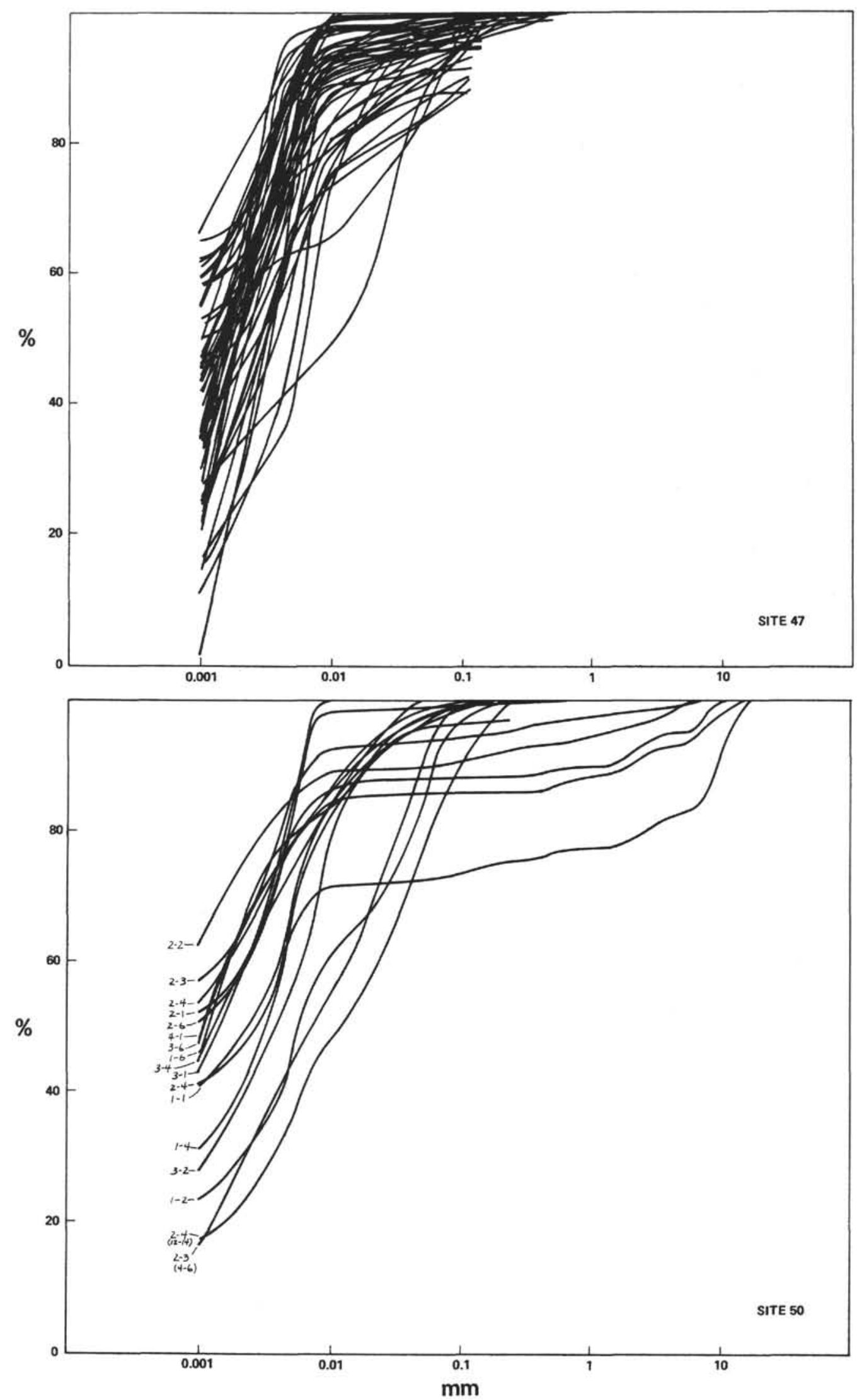

Figure 6. Cumulative curves for samples from Sites 47 and 50. 

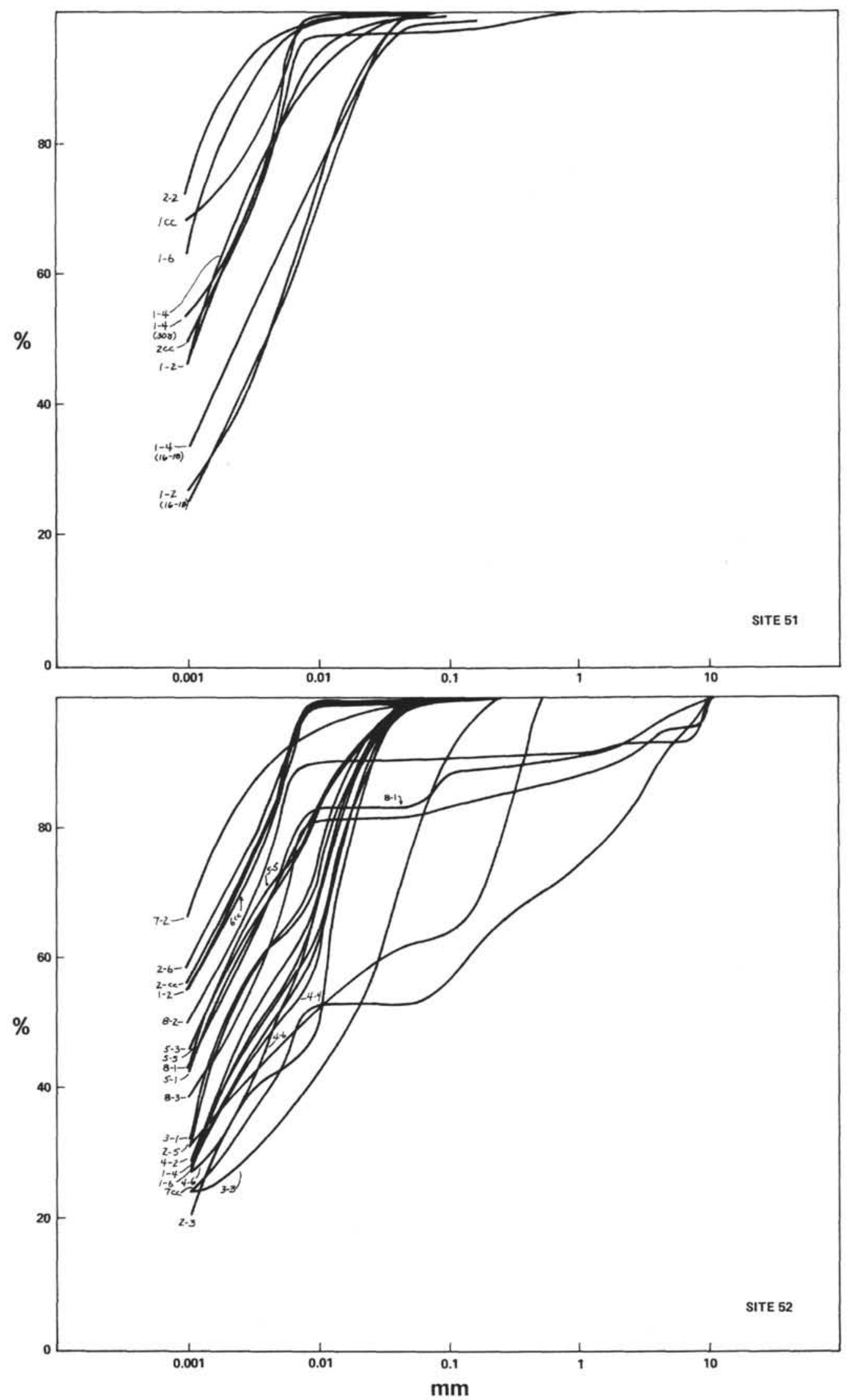

Figure 7. Cumulative curves for samples from Sites 51 and 52. 

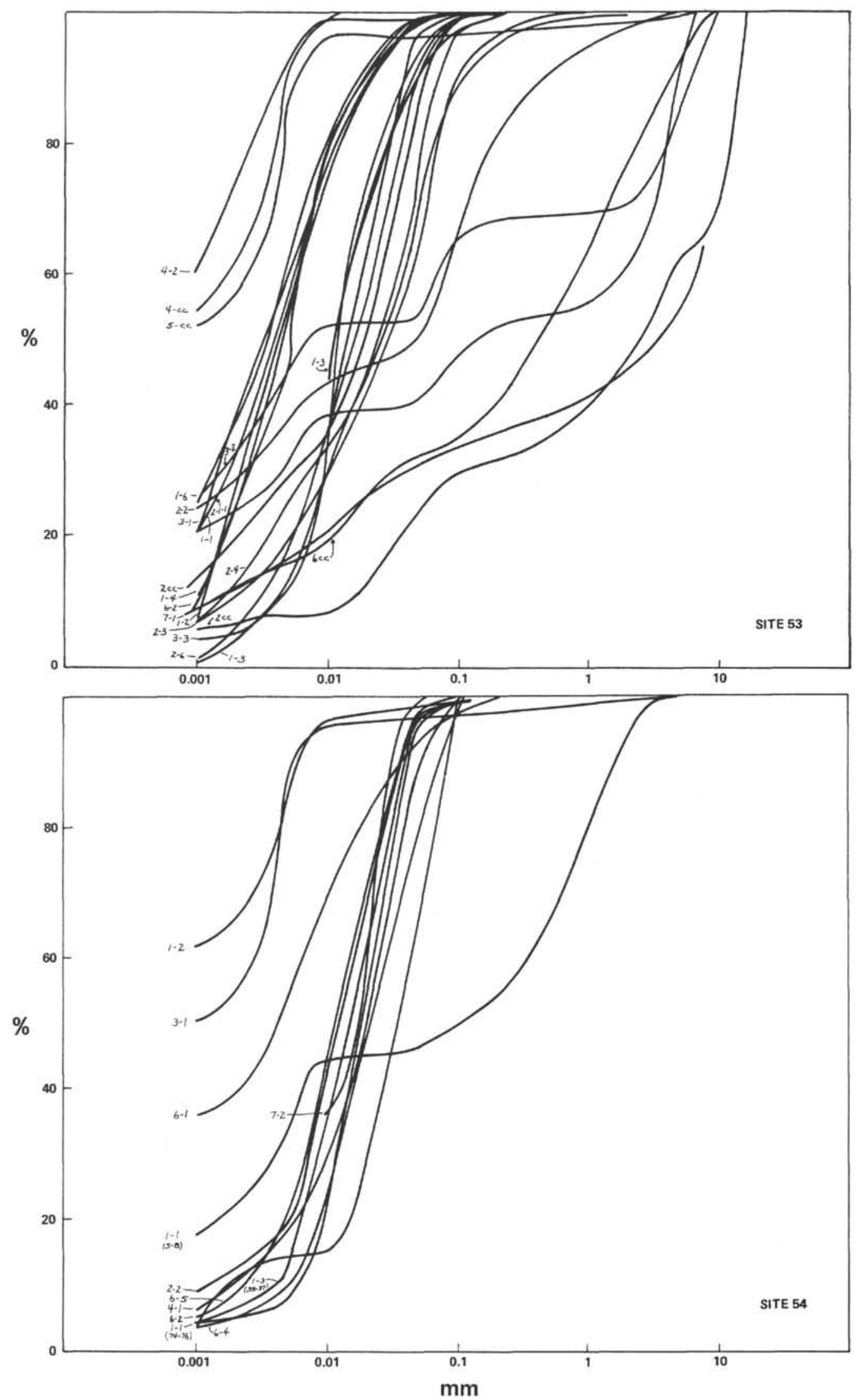

Figure 8. Cumulative curves for samples from Sites 53 and 54. 

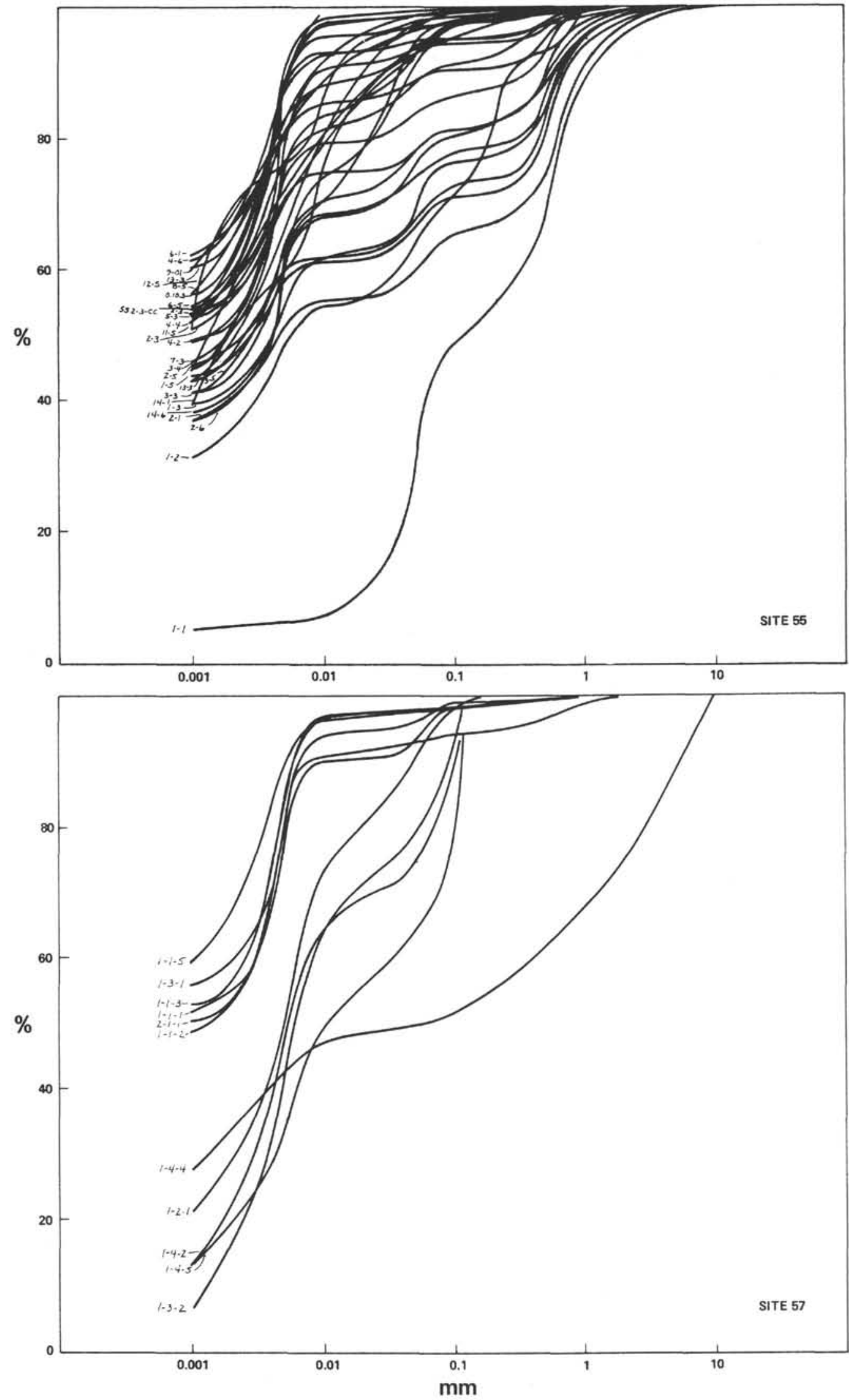

Figure 9. Cumulative curves for samples from Sites 55 and 57. 

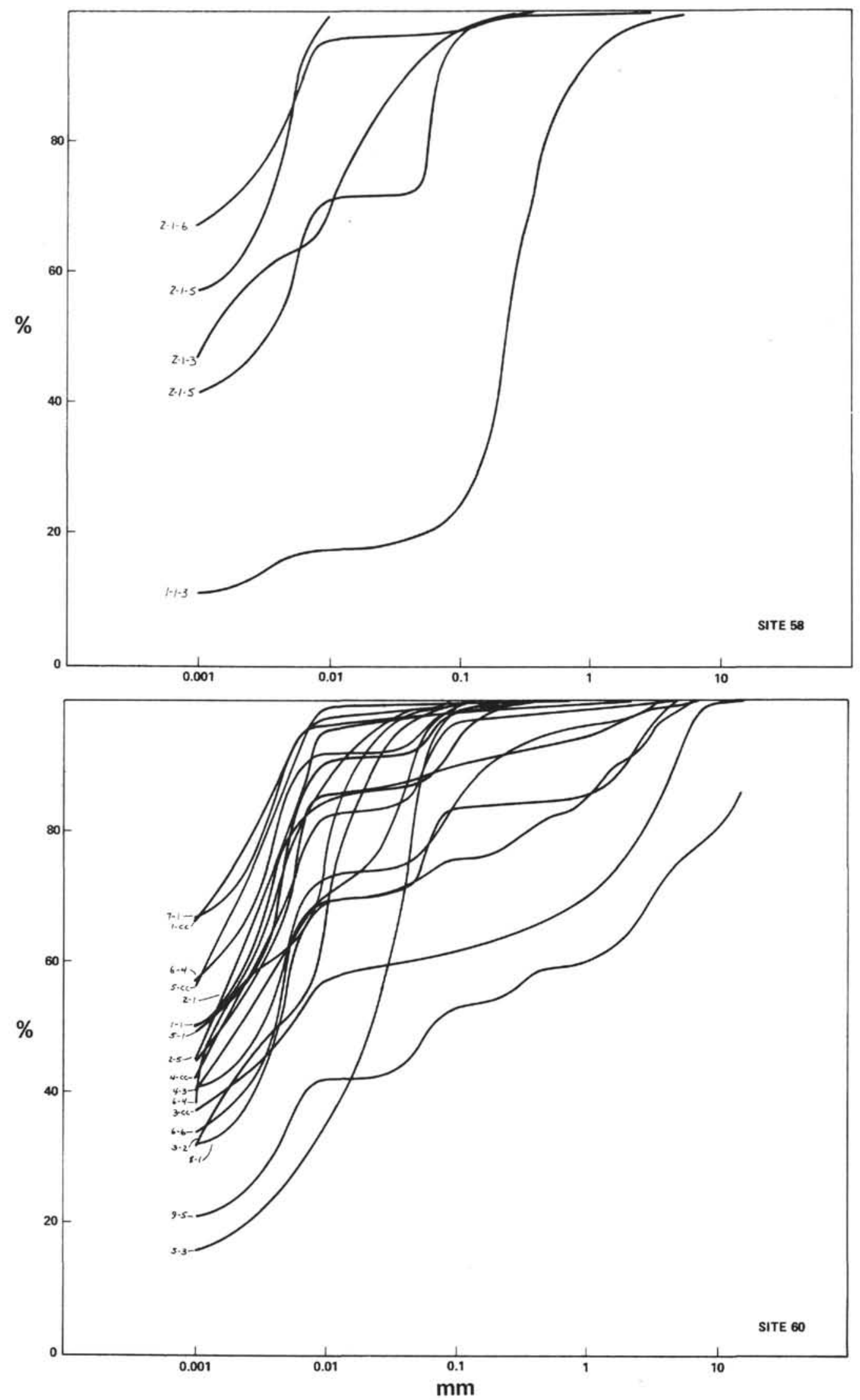

Figure 10. Cumulative curves for samples from Sites 58 and 60. 


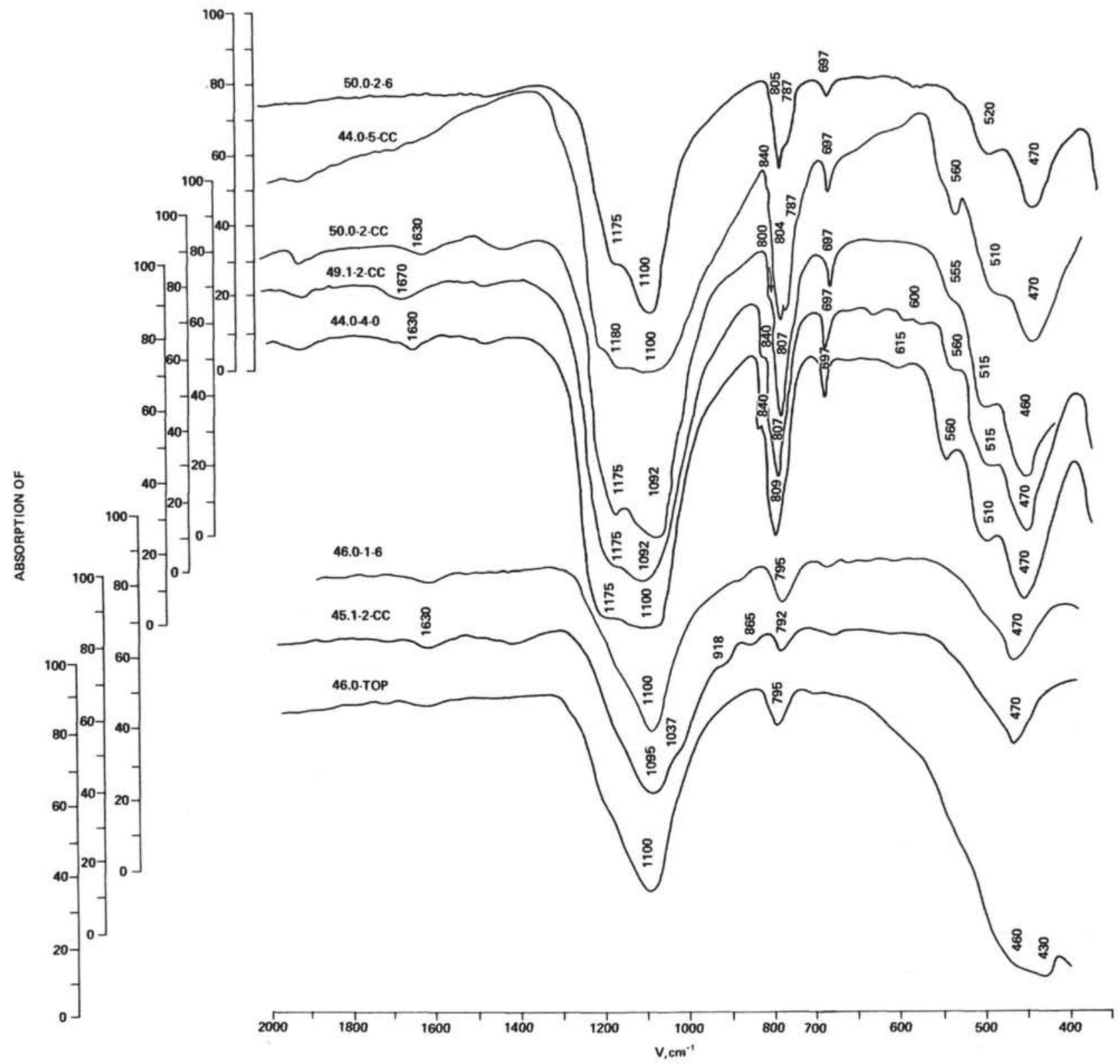

Figure 11. Infra-red spectra results on Leg 6 samples. 

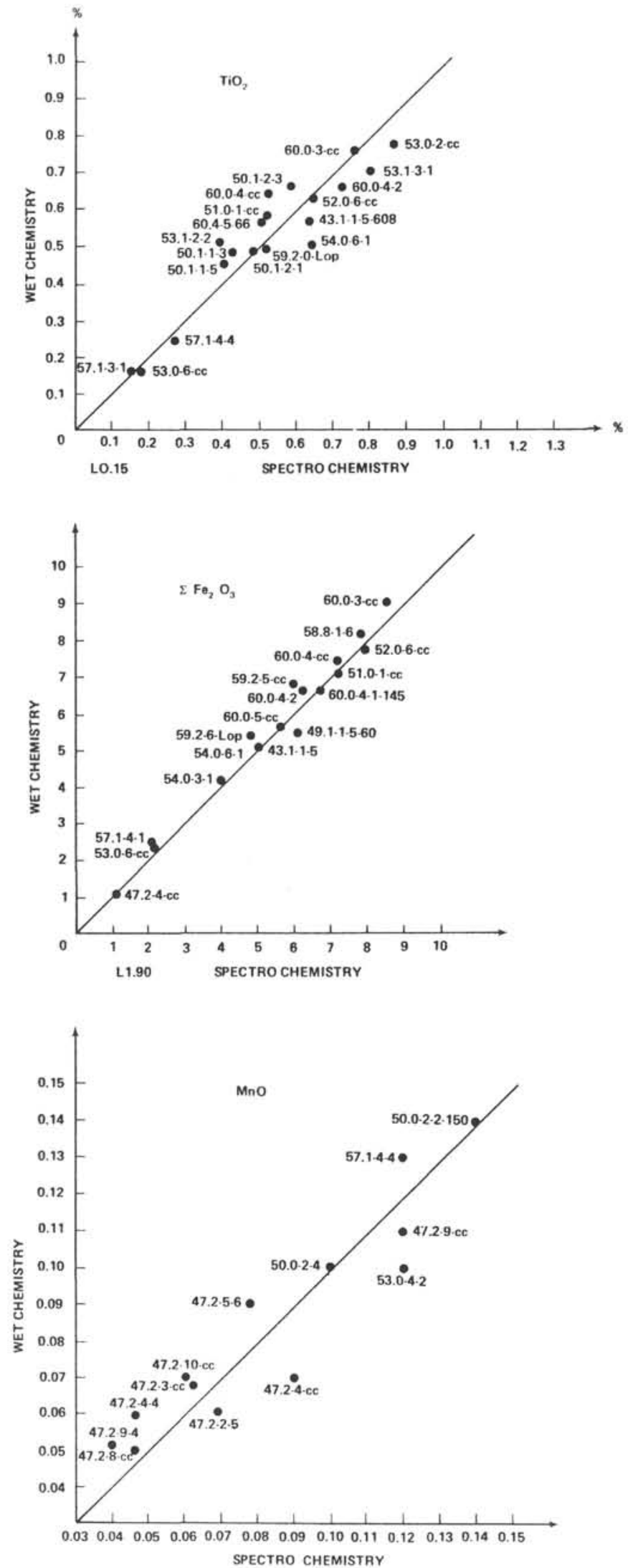

Figure 12. Comparison of analyses for $\mathrm{TiO}_{2}, \mathrm{Fe}_{2} \mathrm{O}_{3}$ and $\mathrm{MnO}$ using wet chemistry and spectrochemistry. 
tridymite, 568 centimeter $^{-1}$. Quartz glass in the region of 1250 to 1100 centimeter $^{-1}$ and 830 to 750 centimeter $^{-1}$, as compared to the corresponding bands of crystalline silica has wider and less intensive absorption bands whose position in the spectrum in most cases coincides almost exactly with the position of similar bands of $\gamma$ - quartz.

Absorption bands of the 1100 centimeter $^{-1}$ region are considered as valent and those of the 460 to 530 centimeter ${ }^{-1}$ region as deformation variations of $\mathrm{SiO}_{4}$-tetrahedra. Bands in the 830 to 750 centimeter $^{-1}$ region correspond to $\mathrm{V}_{5} \mathrm{SiOSi}$ variations (which consist of one or two components formed from the splitting of a fully symmetrical fluctuation of an $\mathrm{SiO}_{4}$-tetrahedron). The splitting of the fluctuation and removal of the degeneration is related to a lowering of the $\mathrm{SiO}_{4}$-tetrahedron symmetry when bands of $\mathrm{SiO}_{4}$-tetrahedrons are formed in the framework silicates, which are $\mathrm{SiO}_{2}$ modifications.

The infrared absorption spectra were recorded in the 2000 to 4000 centimeter $^{-1}$ with the UR-10 infrared spectrophotometer. Fine dispersed powder films on the $\mathrm{KBr}$ basis were prepared by the sedimentation method and used as samples. The thickness of the films expressed arbitrarily in $\mathrm{mg} / \mathrm{cm}^{2}$ was 0.2 to 0.3 .

\section{Results}

The obtained spectra (Figure 11) can be divided into several groups:

(1) Samples 50.0-2-6; 44.0-5-CC-The spectra of these samples are very close to the $\gamma$-quartz spectrum (especially of Sample 50.0-2-6); bands 1167, 1095, 804, $785,695,523$ and 465 centimeter $^{-1}$, all being the bands of $\gamma$-quartz, are detectable. Water is absent almost completely.

Sample 44.0-5-CC has an $\gamma$-quartz spectrum, except for bands 560 centimeter $^{-1}$ and 625 centimeter $^{-1}$ (weak). Such a spectrum is typical of the cryptocrystalline varieties of silica (for instance, chalcedony). An additional band (560 centimeter ${ }^{-1}$ ) can be attributed either to $\gamma$-tridymite admixture, that is, the tridymite type parts of the structure, or to a peculiar deformation of $\mathrm{SiO}_{4}$-tetrahedrons typical of cryptocrystalline varieties of silica. Water is present in the sample in small amounts (to 0.5 of a molecule per each formal unit of silica). Unlike quartz studied previously in dif- ferent deposits, the analyzed sample has a weakly intensive peak of 840 centimeters $^{-1}$.

(2) A group of the samples: 49.1-2-CC, 50.0-2-CC, 44.0-4-0 have spectra similar to that of $\gamma$-quartz (bands $1175,1092,697,515,460$ centimeter $^{-1}$ are detectable) but differing from $\gamma$-quartz in:

(a) new bands of 620 and 560 centimeter $^{-1}$ which, as was already stated, are probably related to crystobalite and "tridymite components";

(b) the average (in relation to the first group of the samples) number of water molecules;

(c) The presence of band 808 centimeter $^{-1}$ with an additional peak- "shoulder" of 837 centimeters $^{-1}$ in the 804 to 785 centimeter $^{-1}$ region instead of $\gamma$-quartz duplicate; the latter variation is the main one not recorded earlier for the natural samples. Such changes are related, most probably, to a noticeable deformation of relations between $\mathrm{Si}-\mathrm{O}-\mathrm{Si}$ and $\mathrm{SiO}_{4}$-tetrahedrons in the crystalline structure of the latter group of the samples.

(3) A number of the samples 45.1-11-CC, 46.0-top, 46.0-1-6, by the type of their spectrum (the presence of bands 1100,795 and 470 centimeter $^{-1}$ ), can be regarded as a considerably "amorphisized" variety of silica. The bands are diffusive and not clearly cut, therefore, relics of $\gamma$-quartz are evidently present; in Sample 45.1-11-CC, admixture bands are found.

\section{DETERMINATION OF DIATOMS ON SAMPLES FROM LEG 6}

(A. P. Jouse, O. G. Kozlova and V. V. Mukhina)

\section{Methods}

Dry sediment is boiled for 15 to 20 minutes in sodium tripolyphosphate $\left(\mathrm{Na}_{5} \mathrm{P}_{3} \mathrm{O}_{10}\right)$ and hydrogen peroxide $\left(\mathrm{H}_{2} \mathrm{O}_{2}-33\right.$ per cent $)$ to disperse the sediment and remove organic admixtures. After boiling, the sediment is washed in distilled water to remove sodium tripolyphosphate. Thus, treated sediment is used to prepare consistent microscope slides by placing the sediment in 1.68. The slides are examined under a biological microscope with $\times 90$ immersion objective. 
Results

\begin{tabular}{|c|c|c|c|c|c|}
\hline Hole & Core & Section & Interval $(\mathrm{cm})$ & Determinations & Age \\
\hline 48.1 & 1 & 4 & $17-19$ & No diatoms were found. & \\
\hline 49.0 & 1 & 1 & $11-13$ & $\begin{array}{l}\text { Among the diatoms there are: Thalassionema } \\
\text { nitzschieides Grun, Ethmodiscus rex Hendey, } \\
\text { Coscinodiscus nodulifer A. S., Planktoniella } \\
\text { sol Schutt., Nitzschia marina Grun. }\end{array}$ & Pleistocene \\
\hline 49.0 & 1 & 4 & $6-8$ & No diatoms were found. & \\
\hline 49.0 & 1 & 5 & $70-72$ & Single spores (moss?) & \\
\hline 49.0 & 2 & 1 & $70-71$ & Mostly coccoliths. & \\
\hline 49.1 & 1 & 1 & $77-79$ & $\begin{array}{l}\text { No diatoms were found. One valve of Gladius (?) } \\
\text { was found. }\end{array}$ & Cretaceous \\
\hline 49.1 & 1 & 2 & $124-126$ & One valve of Stephanopyxis sp. was found. & \\
\hline 49.1 & 1 & 5 & $38-40$ & No diatoms were found. Mostly coccoliths. & \\
\hline 49.1 & 2 & 2 & $145-150$ & Mostly coccoliths. & \\
\hline 50.1 & 1 & 1 & $62-64$ & $\begin{array}{l}\text { Nitzschia praemarina Jouse, Thalassionema } \\
\text { nitzschioides Grun, Th. nitzschioides } v \text {. parva Heiden, } \\
\text { Th. nitzschioides } v \text {. obtusa, Thalassiosira pestriepii } \\
\text { Pr.-Lavr., Nitzschia marina Grun., Th. pesrupii v. } \\
\text { plana Jouse, Coscinodiscus crenulatus Grun., Hemi- } \\
\text { discus cuneiformis v. ventricosa Hust. }\end{array}$ & \\
\hline 50.1 & 1 & 2 & $15-17$ & $\begin{array}{l}\text { Coscinodiscus nodulifer A. S., C. crenulatus Grun., } \\
\text { Nitzschia marina Grun., Thalassionema nitzschioides } \\
\text { v. plana Jouse, Thalassionema nitzschioides Grun., } \\
\text { Th. nit. v. parva. A wide tropical complex of } \\
\text { diatoms. }\end{array}$ & $\begin{array}{l}\text { Early and } \\
\text { Middle } \\
\text { Pleistocene }\end{array}$ \\
\hline 50.1 & 1 & 3 & $7-9$ & $\begin{array}{l}\text { A wide tropical complex of diatoms. The most } \\
\text { typical species of the complex are: Nitzschia } \\
\text { marina Grun., Coscinodiscus crenulatus Grun., } \\
\text { Ethmodiscus rex Hendey, Coscinodiscus africanus } \\
\text { Janisch, C. nodulifer A. S., Hemidiscus cunei- } \\
\text { formus Wall., Planktoniella sol Schutt, Thalas- } \\
\text { siosira lineata Jouse, Thalassionema nitzschioides } \\
\text { Grun., Pseudoeunotia doliolus Grun. }\end{array}$ & $\begin{array}{l}\text { Late } \\
\text { Pleistocene }\end{array}$ \\
\hline 50.1 & 1 & 4 & $17-19$ & $\begin{array}{l}\text { Thalassionema nitzschioides } v \text {. parva Heiden, Th. } \\
\text { nit. } v \text {. obtusa, Thalassiosira pestrupii v. plana Jouse, } \\
\text { Coscinodiscus lineatus Ehr., C. wailesii Grun et } \\
\text { Ougst., Actinocyclus ehrenbergii Ralfs. }\end{array}$ & $\begin{array}{l}\text { Early } \\
\text { Pleistocene }\end{array}$ \\
\hline 50.1 & 1 & 5 & $15-17$ & $\begin{array}{l}\text { The composition of diatoms and their age are the } \\
\text { same as in Sample 50.1-1-2,15-17 centimeters. }\end{array}$ & \\
\hline 50.1 & 1 & 6 & $16-18$ & $\begin{array}{l}\text { The composition and age of diatoms are the same } \\
\text { as in Sample 50.1-1-1, } 62 \text { to } 64 \text { centimeters. }\end{array}$ & \\
\hline 50.1 & 2 & 1 & $130-132$ & $\begin{array}{l}\text { No diatoms were found; single radiolarian shells } \\
\text { were found. }\end{array}$ & \\
\hline 50.1 & 2 & 3 & 4-6 & No diatoms were found. & \\
\hline 50.1 & 2 & 3 & $35-37$ & Single fragments of diatoms cannot be determined. & \\
\hline 50.1 & 2 & 4 & $12-14$ & $\begin{array}{l}\text { No diatoms were found; single sponge spicules } \\
\text { were found. }\end{array}$ & \\
\hline 50.1 & 2 & 5 & $8-10$ & The same as above. & \\
\hline
\end{tabular}




\begin{tabular}{|c|c|c|c|c|c|}
\hline Hole & Core & Section & Interval $(\mathrm{cm})$ & Determinations & Age \\
\hline 50.1 & 3 & 3 & $15-17$ & $\begin{array}{l}\text { No diatoms were found. Single spores of moss were } \\
\text { found. Single Radiolaria poorly preserved. }\end{array}$ & \\
\hline 50.1 & 3 & 5 & $28-30$ & No diatoms were found. & \\
\hline 50.1 & 3 & 2 & $76-78$ & No diatoms were found. & \\
\hline 51.1 & 1 & 1 & $15-17$ & $\begin{array}{l}\text { Wide tropical species are peculiar for the diatom } \\
\text { complex: Thalassiosira oestrupii v. plana Jouse, } \\
\text { Nitzschia praemarina (Jouse), } N \text {. marina f. minor, } \\
\text { N. pliocene Brun., Hemidiscus cuneiformis } f \text {. } \\
\text { ventricosa Hust., Thalassionema nitzschiodes v. } \\
\text { parva Heiden. }\end{array}$ & $\begin{array}{l}\text { Late } \\
\text { Pliocene }\end{array}$ \\
\hline 51.1 & 1 & 2 & $16-18$ & $\begin{array}{l}\text { The number of diatoms and silicoflagellates is less } \\
\text { than in sample } 51.1-1-1,15-17 \text { centimeters. The } \\
\text { composition of diatoms and their age are the same. }\end{array}$ & \\
\hline 51.1 & 1 & 3 & $30-32$ & $\begin{array}{l}\text { The diatom complex includes: Nitzschia praemarina } \\
\text { (Jouse), Thalassiosira oestrupii v. plana Jouse, } \\
\text { Denticula kamchatica Zabelina. }\end{array}$ & $\begin{array}{l}\text { Late } \\
\text { Pliocene }\end{array}$ \\
\hline 51.1 & 1 & 5 & $16-18$ & $\begin{array}{l}\text { The diatom complex includes: Thalassiosira } \\
\text { oestrupii } v \text {. plana Jouse, Nitzschia pliocena } \text { Brun., } \\
\text { N. praemarina (Jouse), } N \text {. marina f. minor, Thalas- } \\
\text { sionema nitzschioides } v \text {. parva Heiden, Denticula } \\
\text { kamchatica Zabelina. }\end{array}$ & $\begin{array}{l}\text { Late } \\
\text { Pliocene }\end{array}$ \\
\hline 51.1 & 2 & 2 & $8-10$ & No diatoms were found. & \\
\hline 59.2 & 2 & 2 & 145 & No diatoms were found. Mostly radiolarians. & \\
\hline 59.2 & 2 & 4 & 4-6 & $\begin{array}{l}\text { Diatoms are poorly preserved, mostly fragments of } \\
\text { Hemialus sp., Asteromphalus sp., Coscinodiscus sp. }\end{array}$ & Miocene (?) \\
\hline 59.2 & 2 & 6 & $16-18$ & No diatoms were found. Mostly radiolarians. & \\
\hline 59.2 & 6 & 6 & $0-5$ & $\begin{array}{l}\text { No diatoms were found. Fragments of Tertiary } \\
\text { radiolarians. }\end{array}$ & \\
\hline 58.2 & 1 & 4 & $31-33$ & $\begin{array}{l}\text { The diatom complex includes single fragments of } \\
\text { Coscinodiscus paleaceus Rattr., Hemiaulus sp., } \\
\text { Cestodiscus sp. }\end{array}$ & Miocene (?) \\
\hline
\end{tabular}


TABLE 1
Mineralogical Analysis of Fraction Greater Than $0.1 \mathrm{~mm}$

\begin{tabular}{|c|c|c|c|c|c|c|c|c|c|c|c|c|c|c|c|c|c|c|c|c|c|c|c|}
\hline 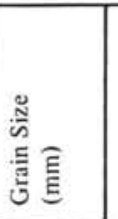 & 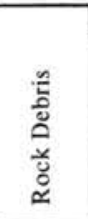 & $\begin{array}{l}\frac{\tilde{u}}{0} \\
\frac{0}{0} \\
\delta\end{array}$ & 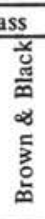 & 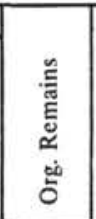 & 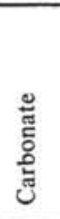 & 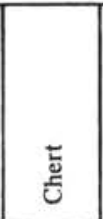 & 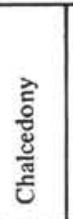 & 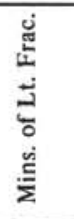 & 嵓 & 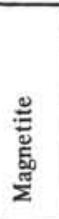 & 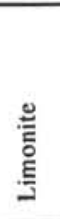 & 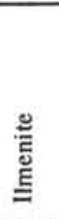 & 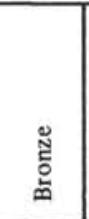 & 茕 & 产 & 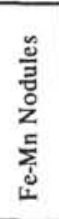 & 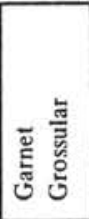 & 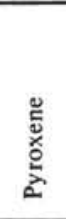 & 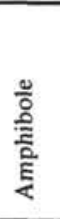 & $\begin{array}{l}\text { 음 } \\
\text { 홉 }\end{array}$ & $\frac{\mathscr{g}}{2}$ & 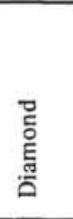 & 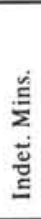 \\
\hline \multicolumn{24}{|c|}{ Hole $44.0-3-\mathrm{CC}$} \\
\hline $0.25-0.1$ & 0.72 & - & - & 36.39 & - & 35.20 & - & - & - & - & - & - & 15.00 & - & 8.80 & - & - & 0.65 & - & 0.80 & - & 2.44 & - \\
\hline $0.5-0.25$ & 0.46 & - & - & 33.30 & - & 34.63 & - & - & - & - & - & - & 25.24 & - & 5.39 & - & - & - & - & - & - & 0.98 & - \\
\hline $1-0.5$ & - & - & - & 12.91 & - & 48.79 & - & - & - & - & - & - & 29.90 & - & 8.40 & - & - & - & - & - & - & - & - \\
\hline $2-1$ & 15.00 & - & - & - & - & 70.00 & - & - & - & - & - & - & 14.00 & - & 1.00 & - & - & - & - & - & - & - & - \\
\hline 3-2 & 15.00 & - & - & - & - & 70.00 & - & - & - & - & - & - & 12.00 & - & 3.0 & - & - & - & - & - & - & - & - \\
\hline $5-3$ & 20.00 & - & - & - & - & 75.00 & - & - & - & - & - & - & 5.00 & - & - & - & - & - & - & - & - & - & - \\
\hline 7.5 & - & - & - & - & - & 100.00 & - & - & - & - & - & - & - & - & - & - & - & - & - & - & - & - & - \\
\hline $10-7$ & - & - & - & - & - & 100.00 & - & - & - & - & - & - & - & - & - & - & - & - & - & - & - & - & - \\
\hline$>10$ & - & - & - & - & - & 100.00 & - & - & - & - & - & - & - & - & - & - & - & - & - & - & - & - & - \\
\hline \multicolumn{24}{|c|}{ Hole $44.0-4-\mathrm{CC}$} \\
\hline $0.25-0.1$ & - & - & - & 100.00 & - & - & - & - & - & - & - & - & - & - & - & - & - & - & - & - & - & - & - \\
\hline $0.5-0.25$ & - & - & - & \begin{tabular}{|l|}
95.67 \\
\end{tabular} & - & 4.00 & - & - & - & - & - & - & 0.33 & - & - & - & - & - & - & - & - & - & - \\
\hline $1-0.5$ & - & - & - & 85.10 & - & 12.30 & - & - & - & - & - & - & 2.60 & - & - & - & - & - & - & - & - & - & - \\
\hline \multicolumn{24}{|c|}{ Hole 47.2-8-CC } \\
\hline $0.25-0.1$ & - & - & - & \begin{tabular}{|l|}
90.70 \\
\end{tabular} & - & - & - & - & 9.30 & - & - & - & - & - & - & - & - & - & - & - & - & - & - \\
\hline $0.5-0.25$ & - & - & - & 100.00 & - & - & - & - & - & - & - & - & - & - & - & - & - & - & - & - & - & - & - \\
\hline 1-0.5 & - & - & - & 4.70 & - & 2.40 & - & - & 92.90 & - & - & - & - & - & - & - & - & - & - & - & - & - & - \\
\hline \multicolumn{24}{|c|}{ Hole $48.2-2-1,145 \mathrm{~cm}$} \\
\hline $0.25-0.1$ & - & - & - & 100.00 & - & - & - & - & - & - & - & - & - & - & - & - & - & - & - & - & - & - & - \\
\hline $0.5-0.25$ & - & - & - & 100.00 & - & - & - & - & - & - & - & - & - & - & - & - & - & - & - & - & - & - & - \\
\hline 1-0.5 & 1.23 & - & - & $\mid 96.30$ & - & 2.47 & - & - & - & - & - & - & - & - & - & - & - & - & - & - & - & - & - \\
\hline \multicolumn{24}{|c|}{ Hole $49.1-1-5,60 \mathrm{~cm}$} \\
\hline $0.25-0.1$ & 18.33 & - & - & 1.33 & - & 0.66 & 2.00 & 2.33 & - & - & - & - & - & 75.35 & - & - & - & - & - & - & - & - & - \\
\hline
\end{tabular}


TABLE 1 - Continued

\begin{tabular}{|c|c|c|c|c|c|c|c|c|c|c|c|c|c|c|c|c|c|c|c|c|c|c|c|}
\hline \multirow[b]{2}{*}{ 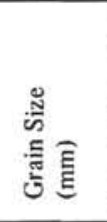 } & \multirow[b]{2}{*}{ 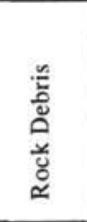 } & \multicolumn{2}{|c|}{ Volc. Glass } & \multirow[b]{2}{*}{ 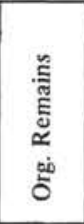 } & \multirow[b]{2}{*}{ 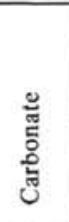 } & \multirow[b]{2}{*}{ 离 } & \multirow[b]{2}{*}{$\begin{array}{l}\text { हू } \\
\text { हूँّ } \\
\text { हूँ } \\
\text { है }\end{array}$} & \multirow[b]{2}{*}{ 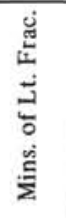 } & \multirow[b]{2}{*}{$\stackrel{\overline{\underline{n}}}{\tilde{\check{u}}}$} & \multirow[b]{2}{*}{ 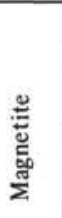 } & \multirow[b]{2}{*}{$\begin{array}{l}\stackrel{\Xi}{\Xi} \\
\stackrel{\Xi}{\Xi}\end{array}$} & \multirow[b]{2}{*}{$\begin{array}{l}\stackrel{\Xi}{\Xi} \\
\stackrel{\Xi}{E}\end{array}$} & \multirow[b]{2}{*}{$\begin{array}{l}\text { N } \\
\text { 롱 }\end{array}$} & \multirow[b]{2}{*}{ 莹 } & \multirow[b]{2}{*}{ 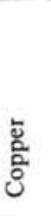 } & \multirow[b]{2}{*}{ 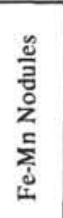 } & \multirow[b]{2}{*}{ 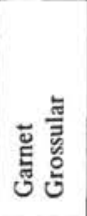 } & \multirow[b]{2}{*}{ 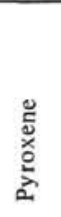 } & \multirow[b]{2}{*}{$\begin{array}{l}\frac{0}{0} \\
\frac{0}{\bar{E}} \\
\frac{\mathrm{E}}{\mathrm{E}}\end{array}$} & \multirow[b]{2}{*}{ 蒙 } & \multirow[b]{2}{*}{$\frac{\mathscr{g}}{\Sigma}$} & \multirow[b]{2}{*}{$\begin{array}{l}\overline{\check{L}} \\
\text { Ĕ } \\
\text { 。̈ }\end{array}$} & \multirow[b]{2}{*}{ 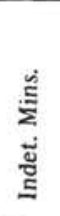 } \\
\hline & & $\frac{\tilde{u}}{\frac{0}{0}}$ & 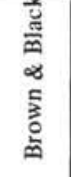 & & & & & & & & & & & & & & & & & & & & \\
\hline \multicolumn{24}{|c|}{ Hole 49.1-1-5, $60 \mathrm{~cm}$ (Continued) } \\
\hline $0.5-0.25$ & 48.62 & - & 1.39 & 4.16 & - & - & - & 4.16 & - & - & - & - & - & 41.67 & - & - & - & - & - & - & - & - & - \\
\hline Hole 50. & -1 , Top & & & & & & & & & & & & & & & & & & & & & & \\
\hline $0.25-0.1$ & 35.66 & - & - & 35.00 & - & 28.82 & 0.17 & - & - & - & - & - & - & 0.35 & - & - & - & - & - & - & - & - & - \\
\hline $0.5-0.25$ & 28.96 & - & - & 10.74 & - & 49.24 & - & - & 9.52 & 0.20 & - & - & 0.19 & 0.55 & - & - & - & - & - & - & - & 0.40 & 0.20 \\
\hline $1-0.5$ & 40.80 & - & - & 1.10 & - & 56.42 & - & - & - & - & - & - & 0.29 & 1.39 & - & - & - & - & - & - & - & - & - \\
\hline $2-1$ & 23.89 & - & - & - & 3.43 & 60.96 & - & - & 7.53 & - & - & - & - & 4.11 & - & - & - & - & - & 0.08 & - & - & - \\
\hline $3-2$ & 9.37 & - & - & - & - & 68.75 & - & - & 21.88 & - & - & - & - & - & - & - & - & - & - & - & - & - & - \\
\hline $5-3$ & 50.00 & - & - & - & - & 50.00 & - & - & - & - & - & - & - & - & - & - & - & - & - & - & - & - & - \\
\hline $10-7$ & - & - & - & - & - & 100.00 & - & - & - & - & - & - & - & - & - & - & - & - & - & - & - & - & - \\
\hline Hole 50. & 2,150 & & & & & & & & & & & & & & & & & & & & & & \\
\hline $0.25-0.1$ & 6.87 & - & - & 18.12 & - & 74.48 & 0.41 & - & - & - & - & - & - & 0.12 & - & - & - & - & - & - & - & - & - \\
\hline $0.5-0.25$ & 12.85 & - & - & 1.95 & - & 84.72 & 0.48 & - & - & - & - & - & - & - & - & - & - & - & - & - & - & - & - \\
\hline $1-0.5$ & 27.69 & - & - & - & - & 62.91 & - & - & 4.22 & - & - & - & - & 4.69 & - & - & - & - & - & 0.49 & - & - & - \\
\hline $2-1$ & 19.23 & - & - & - & - & 73.07 & - & - & 7.70 & - & - & - & - & - & - & - & - & - & - & - & - & - & - \\
\hline $3-2$ & 30.77 & - & - & - & - & 69.23 & - & - & - & - & - & - & - & - & - & - & - & - & - & - & - & - & - \\
\hline $5-3$ & 50.00 & - & - & - & - & 50.00 & - & - & - & - & - & - & - & - & - & - & - & - & - & - & - & - & - \\
\hline Hole 50. & $-4,150$ & & & & & & & & & & & & & & & & & & & & & & \\
\hline $0.25-0.1$ & 7.48 & - & - & 17.78 & - & 74.73 & - & - & - & - & - & - & - & 0.01 & - & - & - & - & - & - & - & - & - \\
\hline $0.5-0.25$ & 19.45 & - & - & 3.94 & - & 72.51 & - & - & - & - & - & - & - & 3.77 & - & - & - & - & - & - & - & 0.13 & 0.20 \\
\hline $1-0.5$ & 7.00 & - & - & - & - & 92.67 & - & - & - & - & - & - & - & - & - & - & - & - & - & 0.33 & - & - & - \\
\hline $2-1$ & 12.20 & - & - & - & - & 87.80 & - & - & - & - & - & - & - & - & - & - & - & - & - & - & - & - & - \\
\hline $3-2$ & 20.00 & - & - & - & - & 80.00 & - & - & - & - & - & - & - & - & - & - & - & - & - & - & - & - & - \\
\hline $5-3$ & - & - & - & - & - & 100.00 & - & - & - & - & - & - & - & - & - & - & - & - & - & - & - & - & - \\
\hline 7.5 & 25.00 & - & - & - & - & 75.00 & - & - & - & - & - & - & - & - & - & - & - & - & - & - & - & - & - \\
\hline
\end{tabular}


TABLE 1 - Continued

\begin{tabular}{|c|c|c|c|c|c|c|c|c|c|c|c|c|c|c|c|c|c|c|c|c|c|c|c|}
\hline \multirow[b]{2}{*}{ 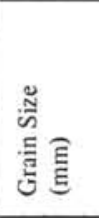 } & \multirow[b]{2}{*}{ 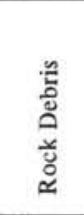 } & \multicolumn{2}{|c|}{$\begin{array}{l}\text { Volc. Glass } \\
\end{array}$} & \multirow[b]{2}{*}{ 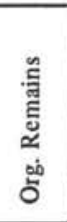 } & \multirow[b]{2}{*}{ 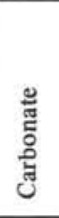 } & \multirow[b]{2}{*}{ 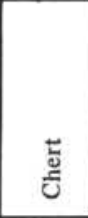 } & \multirow[b]{2}{*}{ 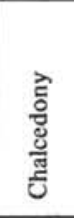 } & \multirow[b]{2}{*}{ 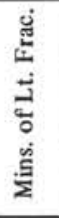 } & \multirow[b]{2}{*}{ 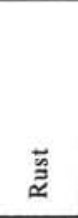 } & \multirow[b]{2}{*}{ 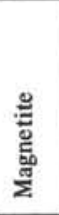 } & \multirow[b]{2}{*}{ 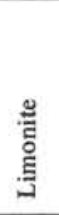 } & \multirow[b]{2}{*}{$\begin{array}{l}\stackrel{\mathscr{\Xi}}{\Xi} \\
\stackrel{E}{E}\end{array}$} & \multirow[b]{2}{*}{ 芯 } & \multirow[b]{2}{*}{ 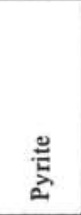 } & \multirow[b]{2}{*}{ 荾 } & \multirow[b]{2}{*}{ 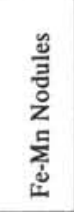 } & \multirow[b]{2}{*}{ 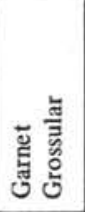 } & \multirow[b]{2}{*}{ 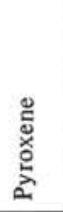 } & \multirow[b]{2}{*}{ 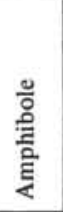 } & \multirow[b]{2}{*}{ 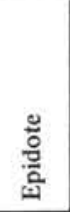 } & \multirow[b]{2}{*}{$\stackrel{\check{\Sigma}}{\check{\Sigma}}$} & \multirow[b]{2}{*}{ 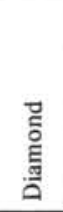 } & \multirow[b]{2}{*}{ 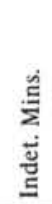 } \\
\hline & & $\frac{\tilde{3}}{0}$ & 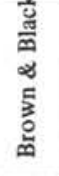 & & & & & & & & & & & & & & & & & & & & \\
\hline \multicolumn{24}{|c|}{ Hole 50.0-2-4, $150 \mathrm{~cm}$ (Continued) } \\
\hline $10-7$ & - & - & - & - & - & 100.00 & - & - & - & - & - & - & - & - & - & - & - & - & - & - & - & - & - \\
\hline$>10$ & - & - & - & - & - & 100.00 & - & - & - & - & - & - & - & - & - & - & - & - & - & - & - & - & - \\
\hline Hole 50. & $2-6,150$ & & & & & & & & & & & & & & & & & & & & & & \\
\hline $0.25-0.1$ & 52.42 & - & - & 8.75 & - & 30.32 & 0.11 & - & 3.13 & - & - & - & 4.02 & 1.15 & - & - & - & - & - & - & - & - & 0.10 \\
\hline $0.5-0.25$ & 33.27 & - & - & 2.80 & - & 54.37 & 0.31 & - & - & - & - & - & 3.28 & 5.57 & - & 0.10 & - & - & - & 0.10 & - & - & 0.20 \\
\hline $1-0.5$ & 28.91 & - & - & 0.60 & - & 62.06 & - & - & 5.26 & - & - & - & - & 2.29 & - & - & - & - & - & 0.88 & - & - & - \\
\hline $2-1$ & 15.24 & - & - & - & - & 81.44 & - & - & 0.95 & - & - & - & - & 1.90 & - & - & - & - & - & 0.77 & - & - & - \\
\hline 3-2 & 4.35 & - & - & - & - & 84.78 & - & - & 4.34 & - & - & - & - & 6.53 & - & - & - & - & - & - & - & - & - \\
\hline $5-3$ & 20.00 & - & - & - & - & 80.00 & - & - & - & - & - & - & - & - & - & - & - & - & - & - & - & - & - \\
\hline 7.5 & 33.33 & - & - & - & - & 66.67 & - & - & - & - & - & - & - & - & - & - & - & - & - & - & - & - & - \\
\hline$>10$ & - & - & - & - & - & 100.00 & - & - & - & - & - & - & - & - & - & - & - & - & - & - & - & - & - \\
\hline Hole 52. & $8-4,145$ & & & & & & & & & & & & & & & & & & & & & & \\
\hline $0.25-0.1$ & 95.67 & 0.33 & - & 3.33 & - & - & - & - & - & - & - & - & - & 0.67 & - & - & - & - & - & - & - & - & - \\
\hline $0.5-0.25$ & 96.34 & 1.33 & - & - & - & 0.67 & - & - & - & - & - & - & - & 1.66 & - & - & - & - & - & - & - & - & - \\
\hline $1-0.5$ & 96.00 & - & - & - & - & 2.00 & - & - & 1.00 & - & - & - & - & 1.00 & - & - & - & - & - & - & - & - & - \\
\hline $2-1$ & 97.56 & - & - & - & - & 2.44 & - & - & - & - & - & - & - & - & - & - & - & - & - & - & - & - & - \\
\hline $3-2$ & 93.94 & - & - & - & - & 6.06 & - & - & - & - & - & - & - & - & - & - & - & - & - & - & - & - & - \\
\hline $5-3$ & 100.00 & - & - & - & - & - & - & - & - & - & - & - & - & - & - & - & - & - & - & - & - & - & - \\
\hline $7-5$ & 100.00 & - & - & - & - & - & - & - & - & - & - & - & - & - & - & - & - & - & - & - & - & - & - \\
\hline $10-7$ & 100.00 & - & - & - & - & - & - & - & - & - & - & - & - & - & - & - & - & - & - & - & - & - & - \\
\hline Hole 52. & $8-5,145$ & & & & & & & & & & & & & & & & & & & & & & \\
\hline $0.25-0.1$ & 97.34 & - & - & 1.66 & - & 0.33 & 0.34 & - & - & - & - & - & - & 0.33 & - & - & - & - & - & - & - & - & - \\
\hline $0.5-0.25$ & 97.68 & - & - & - & - & 6.66 & - & - & - & - & - & - & - & 0.66 & - & - & - & - & - & - & - & - & - \\
\hline
\end{tabular}


TABLE 1 - Continued

\begin{tabular}{|c|c|c|c|c|c|c|c|c|c|c|c|c|c|c|c|c|c|c|c|c|c|c|c|}
\hline 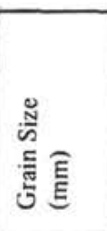 & 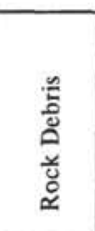 & $\frac{\ddot{3}}{\frac{0}{0}}$ & 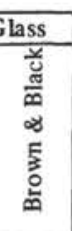 & 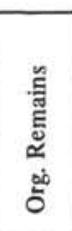 & 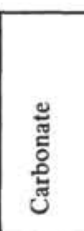 & 힐 & 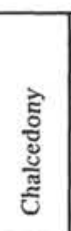 & 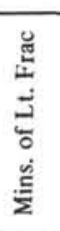 & 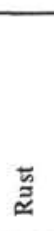 & 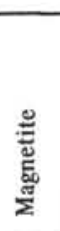 & 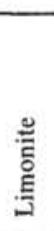 & 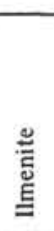 & 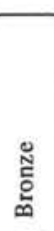 & 总 & 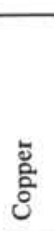 & 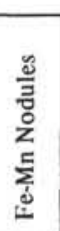 & 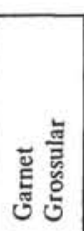 & 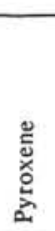 & 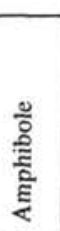 & 흠 & $\frac{\pi}{2}$ & 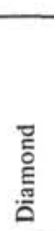 & 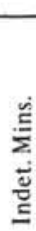 \\
\hline \multicolumn{24}{|c|}{ Hole $52.0-8-5,145 \mathrm{~cm}$ (Continued) } \\
\hline $1-0.5$ & 98.34 & - & - & - & - & 1.66 & - & - & - & - & - & - & - & - & - & - & - & - & - & - & - & - & - \\
\hline $2-1$ & 99.00 & - & - & - & - & 1.00 & - & - & - & - & - & - & - & - & - & - & - & - & - & - & - & - & - \\
\hline 3-2 & 96.00 & - & - & - & - & 4.00 & - & - & - & - & - & - & - & - & - & - & - & - & - & - & - & - & - \\
\hline $5-3$ & 95.24 & - & - & - & - & 4.76 & - & - & - & - & - & - & - & - & - & - & - & - & - & - & - & - & - \\
\hline $7-5$ & 100.00 & - & - & - & - & - & - & - & - & - & - & - & - & - & - & - & - & - & - & - & - & - & - \\
\hline \multicolumn{24}{|c|}{ Hole $53.0-2-\mathrm{CC}$} \\
\hline $0.25-0.1$ & 98.67 & 0.33 & 0.33 & 0.67 & - & - & - & - & - & - & - & - & - & - & - & - & - & - & - & - & - & - & - \\
\hline $0.5-0.25$ & 100.00 & - & - & - & - & - & - & - & - & - & - & - & - & - & - & - & - & - & - & - & - & - & - \\
\hline $1-0.5$ & 100.00 & - & - & - & - & - & - & - & - & - & - & - & - & - & - & - & - & - & - & - & - & - & - \\
\hline $2-1$ & 100.00 & - & - & - & - & - & - & - & - & - & - & - & - & - & - & - & - & - & - & - & - & - & - \\
\hline 3-2 & 100.00 & - & - & - & - & - & - & - & - & - & - & - & - & - & - & - & - & - & - & - & - & - & - \\
\hline $5-3$ & 100.00 & - & - & - & - & - & - & - & - & - & - & - & - & - & - & - & - & - & - & - & - & - & - \\
\hline 7.5 & 100.00 & - & - & - & - & - & - & - & - & - & - & - & - & - & - & - & - & - & - & - & - & - & - \\
\hline $10-7$ & 100.00 & - & - & - & - & - & - & - & - & - & - & - & - & - & - & - & - & - & - & - & - & - & - \\
\hline$>10$ & 100.00 & - & - & - & - & - & - & - & - & - & - & - & - & - & - & - & - & - & - & - & - & - & - \\
\hline \multicolumn{24}{|c|}{ Hole $53.0-7-1,150 \mathrm{~cm}$} \\
\hline $0.25-0.1$ & 99.23 & 0.04 & - & - & 0.40 & - & 0.33 & - & - & - & - & - & - & - & - & - & - & - & - & - & - & - & - \\
\hline $0.5-0.25$ & 98.66 & 0.14 & - & - & 1.20 & - & - & - & - & - & - & - & - & - & - & - & - & - & - & - & - & - & - \\
\hline $1-0.5$ & 89.68 & - & - & 0.16 & 10.00 & - & 0.16 & - & - & - & - & - & - & - & - & - & - & - & - & - & - & - & - \\
\hline $2-1$ & 95.17 & - & - & 2.23 & 2.60 & - & - & - & - & - & - & - & - & - & - & - & - & - & - & - & - & - & - \\
\hline 3-2 & 90.48 & - & - & 4.76 & 4.76 & - & - & - & - & - & - & - & - & - & - & - & - & - & - & - & - & - & - \\
\hline $5-3$ & 100.00 & - & - & - & - & - & - & - & - & - & - & - & - & - & - & - & - & - & - & - & - & - & - \\
\hline $7-5$ & 100.00 & - & - & - & - & - & - & - & - & - & - & - & - & - & - & - & - & - & - & - & - & - & - \\
\hline $10-7$ & 100.00 & - & - & - & - & - & - & - & - & - & - & - & - & - & - & - & - & - & - & - & - & - & - \\
\hline$>10$ & 100.00 & - & - & - & - & - & - & - & - & - & - & - & - & - & - & - & - & - & - & - & - & - & - \\
\hline
\end{tabular}


TABLE 1 - Continued

\begin{tabular}{|c|c|c|c|c|c|c|c|c|c|c|c|c|c|c|c|c|c|c|c|c|c|c|c|}
\hline \multirow[b]{2}{*}{ 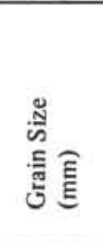 } & \multirow[b]{2}{*}{ 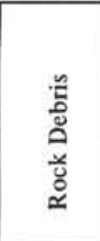 } & \multicolumn{2}{|c|}{ Volc. Glass } & \multirow[b]{2}{*}{ 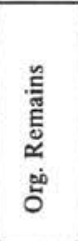 } & \multirow[b]{2}{*}{ 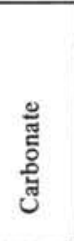 } & \multirow[b]{2}{*}{$\begin{array}{l}\text { L్ } \\
\text { Ẽ }\end{array}$} & \multirow[b]{2}{*}{$\begin{array}{l}\text { ते } \\
\text { हूँ } \\
\text { हूँ } \\
\text { हू }\end{array}$} & \multirow[b]{2}{*}{ 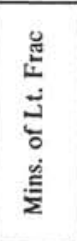 } & \multirow[b]{2}{*}{$\stackrel{\overline{\underline{u}}}{\simeq}$} & \multirow[b]{2}{*}{ 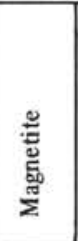 } & \multirow[b]{2}{*}{ 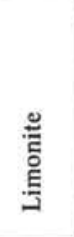 } & \multirow[b]{2}{*}{$\begin{array}{l}\stackrel{\mathscr{E}}{5} \\
\stackrel{\Xi}{E}\end{array}$} & \multirow[b]{2}{*}{ 芯 } & \multirow[b]{2}{*}{ 气 } & \multirow[b]{2}{*}{$\begin{array}{l}\text { o. } \\
\text { ò }\end{array}$} & \multirow[b]{2}{*}{ 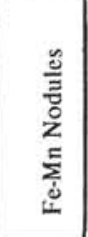 } & \multirow[b]{2}{*}{ 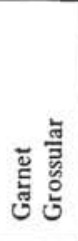 } & \multirow[b]{2}{*}{ 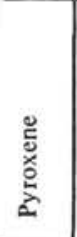 } & \multirow[b]{2}{*}{ 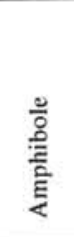 } & \multirow[b]{2}{*}{ 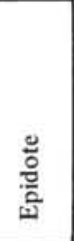 } & \multirow[b]{2}{*}{$\stackrel{\Xi}{\Sigma}$} & \multirow[b]{2}{*}{ 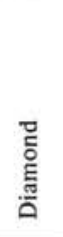 } & \multirow[b]{2}{*}{$\begin{array}{l}\text { 离 } \\
\text { 苛 }\end{array}$} \\
\hline & & $\frac{\frac{y}{3}}{\frac{b}{0}}$ & 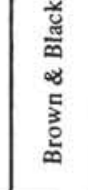 & & & & & & & & & & & & & & & & & & & & \\
\hline \multicolumn{24}{|c|}{ Hole $53.1-2-2,145 \mathrm{~cm}$} \\
\hline $0.25-0.1$ & 23.00 & 44.32 & 16.10 & 0.08 & 0.17 & - & - & 15.73 & - & - & - & - & - & - & - & - & - & - & - & - & - & - & - \\
\hline $0.5-0.25$ & 2.77 & 86.20 & 2.84 & - & - & - & - & 8.19 & - & - & - & - & - & - & - & - & - & - & - & - & - & - & - \\
\hline $1-0.5$ & 3.33 & 94.67 & 0.66 & - & - & - & - & $\mid 0.67$ & - & - & - & - & - & - & - & - & - & 0.67 & - & - & - & - & - \\
\hline $2-1$ & 4.00 & 93.00 & 1.00 & - & - & - & - & $\mid 0.67$ & - & - & - & - & - & - & - & 0.66 & - & 0.67 & - & - & - & - & - \\
\hline $3-2$ & 25.00 & 66.66 & - & - & - & - & - & - & - & - & - & - & - & - & - & - & - & 8.34 & - & - & - & - & - \\
\hline $5-3$ & 40.00 & 40.00 & - & - & - & - & - & - & - & - & - & - & - & - & - & 20.00 & - & - & - & - & - & - & - \\
\hline \multicolumn{24}{|c|}{ Hole $53.1-2-3,16 \mathrm{~cm}$} \\
\hline $0.25-0.1$ & 13.90 & 55.76 & 25.36 & - & - & - & 2.30 & - & - & - & 0.85 & - & - & - & 0.01 & & 0.11 & - & - & - & - & - & - \\
\hline $0.5-0.25$ & 4.88 & 92.04 & 3.08 & - & - & - & - & - & - & - & - & - & - & - & - & - & - & - & - & - & - & - & - \\
\hline Hole 54.0 & & & & & & & & & & & & & & & & & & & & & & & \\
\hline $0.25-0.1$ & 33.00 & 19.00 & 16.28 & 1.03 & - & - & - & 27.20 & 0.03 & 2.03 & - & 0.01 & - & - & - & - & - & 1.42 & - & - & - & - & - \\
\hline $0.5-0.25$ & 40.32 & 19.09 & 15.70 & 0.62 & - & - & - & 20.06 & 0.08 & 0.12 & - & - & - & - & - & - & - & 3.98 & 0.01 & 0.01 & 0.01 & - & - \\
\hline $1-0.5$ & 46.53 & 18.91 & 11.73 & 0.28 & - & - & - & 22.43 & 0.03 & - & - & - & - & - & - & - & - & 0.11 & - & - & - & - & - \\
\hline $2-1$ & 69.00 & 21.66 & 8.34 & 0.34 & - & - & - & $\mid 0.66$ & - & - & - & - & - & - & - & - & - & - & - & - & - & - & - \\
\hline $3-2$ & 100.00 & - & - & - & - & - & - & - & - & - & - & - & - & - & - & - & - & - & - & - & - & - & - \\
\hline $5-3$ & 100.00 & - & - & - & - & - & - & - & - & - & - & - & - & - & - & - & - & - & - & - & - & - & - \\
\hline Hole 54.0 & $6-2,16 \mathrm{cl}$ & & & & & & & & & & & & & & & & & & & & & & \\
\hline $0.25-0.1$ & 0.74 & 95.73 & 3.53 & - & - & - & - & - & - & - & - & - & - & - & - & - & - & - & - & - & - & - & - \\
\hline Hole 54.0 & $6-4,16 \mathrm{c}$ & & & & & & & & & & & & & & & & & & & & & & \\
\hline $0.25-0.1$ & 11.08 & 81.18 & 4.00 & - & - & - & 3.41 & - & - & - & - & - & - & - & - & 0.33 & - & - & - & - & - & - & - \\
\hline Hole 57.1 & $4-4,145$ & & & & & & & & & & & & & & & & & & & & & & \\
\hline $0.25-0.1$ & 3.30 & - & 88.00 & 8.70 & - & - & - & - & - & - & - & - & - & - & - & - & - & - & - & - & - & - & - \\
\hline
\end{tabular}


TABLE 1 - Continued

\begin{tabular}{|c|c|c|c|c|c|c|c|c|c|c|c|c|c|c|c|c|c|c|c|c|c|c|c|}
\hline \multirow[b]{2}{*}{ 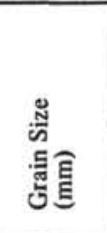 } & \multirow[b]{2}{*}{ 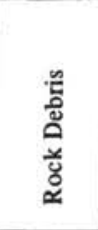 } & \multicolumn{2}{|c|}{ Volc. Glass } & \multirow[b]{2}{*}{ 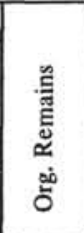 } & \multirow[b]{2}{*}{ 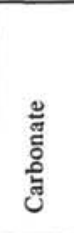 } & \multirow[b]{2}{*}{ 总 } & \multirow[b]{2}{*}{$\begin{array}{l}\text { टे } \\
\text { हूँ } \\
\text { हुँ } \\
\text { है }\end{array}$} & \multirow[b]{2}{*}{ 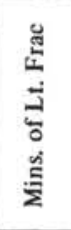 } & \multirow[b]{2}{*}{$\overline{\tilde{a}}$} & \multirow[b]{2}{*}{ 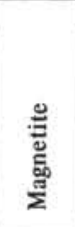 } & \multirow[b]{2}{*}{ 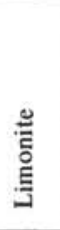 } & \multirow[b]{2}{*}{ 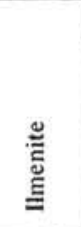 } & \multirow[b]{2}{*}{$\begin{array}{l}\text { స్ } \\
\text { ్ㅗㅁ }\end{array}$} & \multirow[b]{2}{*}{$\stackrel{\Xi}{2}$} & \multirow[b]{2}{*}{$\begin{array}{l}\text { 㟧 } \\
\text { o }\end{array}$} & \multirow[b]{2}{*}{ 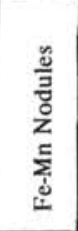 } & \multirow[b]{2}{*}{ 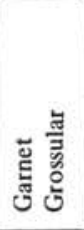 } & \multirow[b]{2}{*}{ 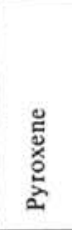 } & \multirow[b]{2}{*}{ 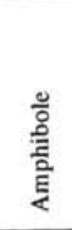 } & \multirow[b]{2}{*}{ 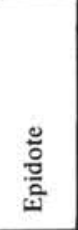 } & \multirow[b]{2}{*}{ 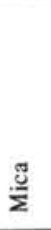 } & \multirow[b]{2}{*}{ 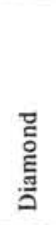 } & \multirow[b]{2}{*}{ 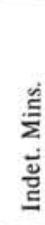 } \\
\hline & & 产 & 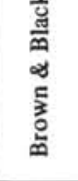 & & & & & & & & & & & & & & & & & & & & \\
\hline Hole 57.1 & $-4-4,145$ & $\mathrm{~cm}$ (Con & inued) & & & & & & & & & & & & & & & & & & & & \\
\hline $0.5-0.25$ & 85.70 & 9. & & 5.30 & - & - & - & - & - & - & - & - & - & - & - & - & - & - & - & - & - & - & - \\
\hline $1-0.5$ & 99.30 & 0. & & - & - & - & - & - & - & - & - & - & - & - & - & - & - & - & - & - & - & - & - \\
\hline $2-1$ & 100.00 & - & - & - & - & - & - & - & - & - & - & - & - & - & - & - & - & - & - & - & - & - & - \\
\hline $3-2$ & 100.00 & - & - & - & - & - & - & - & - & - & - & - & - & - & - & - & - & - & - & - & - & - & - \\
\hline $5-3$ & 100.00 & - & - & - & - & - & - & - & - & - & - & - & - & - & - & - & - & - & - & - & - & - & - \\
\hline $7-5$ & 100.00 & - & - & - & - & - & - & - & - & - & - & - & - & - & - & - & - & - & - & - & - & - & - \\
\hline $10-7$ & 100.00 & - & - & - & - & - & - & - & - & - & - & - & - & - & - & - & - & - & - & - & - & - & - \\
\hline Hole 58. & 3,145 & & & & & & & & & & & & & & & & & & & & & & \\
\hline $0.25-0.1$ & 12.87 & - & 0.60 & 78.08 & 8.12 & - & - & - & - & - & - & - & - & - & - & 0.11 & - & 0.22 & - & - & - & - & - \\
\hline $0.5-0.25$ & 15.26 & 0.01 & 0.53 & 76.89 & 7.13 & - & - & - & - & - & - & - & - & - & - & 0.05 & - & 0.13 & - & - & - & - & - \\
\hline $1-0.5$ & 25.89 & 0.08 & 0.88 & 62.93 & 9.80 & 0.08 & 0.30 & - & - & - & - & - & - & 0.04 & - & - & - & - & - & - & - & - & - \\
\hline $2-1$ & 86.33 & 0.68 & - & 10.66 & - & 2.33 & - & - & - & - & - & - & - & - & - & - & - & - & - & - & - & - & - \\
\hline $3-2$ & 75.00 & - & - & 12.50 & - & 12.50 & - & - & - & - & - & - & - & - & - & - & - & - & - & - & - & - & - \\
\hline $5-3$ & 100.00 & - & - & - & - & - & - & - & - & - & - & - & - & - & - & - & - & - & - & - & - & - & - \\
\hline Hole 60.0 & $2-1,87 c$ & & & & & & & & & & & & & & & & & & & & & & \\
\hline $0.25-0.1$ & 0.62 & 58.31 & 0.35 & - & - & - & 30.31 & - & - & 2.20 & - & - & - & - & 0.01 & 8.18 & 0.02 & - & - & - & - & - & - \\
\hline $0.5-0.25$ & 1.49 & 85.86 & 0.33 & 1.34 & - & - & 10.82 & - & - & - & - & - & - & - & - & - & - & - & - & - & - & - & - \\
\hline Hole 60.0 & $3-\mathrm{CC}$ & & & & & & & & & & & & & & & & & & & & & & \\
\hline $0.25-0.1$ & 88.44 & 0.64 & 2.58 & 0.64 & - & - & - & 4.83 & 0.03 & 1.00 & - & 0.02 & - & - & - & - & - & 1.76 & 0.04 & 0.02 & - & - & - \\
\hline $0.5-0.25$ & 99.00 & 0.33 & 0.33 & - & - & - & - & 0.34 & - & - & - & - & - & - & - & - & - & - & - & - & - & - & - \\
\hline $1-0.5$ & 100.00 & - & - & - & - & - & - & - & - & - & - & - & - & - & - & - & - & - & - & - & - & - & - \\
\hline $2-1$ & 100.00 & - & - & - & - & - & - & - & - & - & - & - & - & - & - & - & - & - & - & - & - & - & - \\
\hline 3-2 & 100.00 & - & - & - & - & - & - & - & - & - & - & - & - & - & - & - & - & - & - & - & - & - & - \\
\hline $5-3$ & 100.00 & - & - & - & - & - & - & - & - & - & - & - & - & - & - & - & - & - & - & - & - & - & - \\
\hline $7-5$ & 100.00 & - & - & - & - & - & - & - & - & - & - & - & - & - & - & - & - & - & - & - & - & - & - \\
\hline
\end{tabular}


TABLE 1 - Continued

\begin{tabular}{|c|c|c|c|c|c|c|c|c|c|c|c|c|c|c|c|c|c|c|c|c|c|c|c|}
\hline \multirow[b]{2}{*}{ 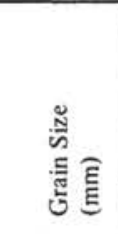 } & \multirow[b]{2}{*}{ 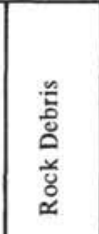 } & \multicolumn{2}{|c|}{$\begin{array}{l}\text { Volc. Glass } \\
\end{array}$} & \multirow[b]{2}{*}{ 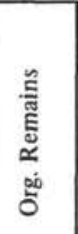 } & \multirow[b]{2}{*}{ 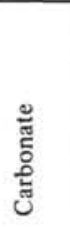 } & \multirow[b]{2}{*}{ 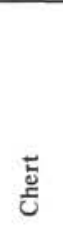 } & \multirow[b]{2}{*}{ 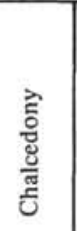 } & \multirow[b]{2}{*}{ 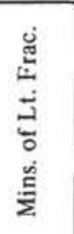 } & \multirow[b]{2}{*}{$\overline{\underline{\underline{y}}}$} & \multirow[b]{2}{*}{ 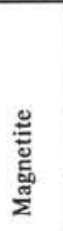 } & \multirow[b]{2}{*}{ 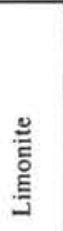 } & \multirow[b]{2}{*}{ 莽 } & \multirow[b]{2}{*}{ 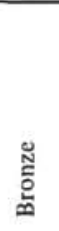 } & \multirow[b]{2}{*}{ 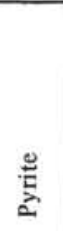 } & \multirow[b]{2}{*}{$\begin{array}{l}\text { dू. } \\
\text { 衣 }\end{array}$} & \multirow[b]{2}{*}{ 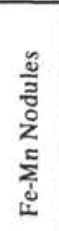 } & \multirow[b]{2}{*}{ 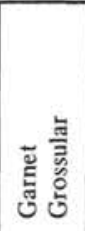 } & \multirow[b]{2}{*}{ 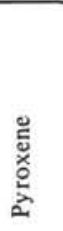 } & \multirow[b]{2}{*}{$\begin{array}{l}\frac{2}{0} \\
\text { 递 } \\
\text { 产 }\end{array}$} & \multirow[b]{2}{*}{ 䒕 } & \multirow[b]{2}{*}{$\frac{\mathscr{J}}{\Sigma}$} & \multirow[b]{2}{*}{ 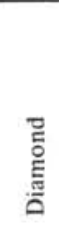 } & \\
\hline & & $\begin{array}{l}\frac{\tilde{c}}{0} \\
\frac{0}{0} \\
\delta\end{array}$ & 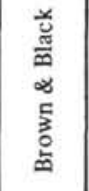 & & & & & & & & & & & & & & & & & & & & 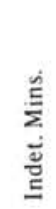 \\
\hline \multicolumn{24}{|c|}{ Hole $60.0-3-\mathrm{CC}$ (Continued) } \\
\hline $10-7$ & 100.00 & - & - & - & - & - & - & - & - & - & - & - & - & - & - & - & - & - & - & - & - & - & - \\
\hline$>10$ & 100.00 & - & - & - & - & - & - & - & - & - & - & - & - & - & - & - & - & - & - & - & - & - & - \\
\hline Hole 60.0 & $-4-\mathrm{CC}$ & & & & & & & & & & & & & & & & & & & & & & \\
\hline $0.25-0.1$ & 84.66 & 11.00 & 4.34 & - & - & - & - & - & - & - & - & - & - & - & - & - & - & - & - & - & - & - & - \\
\hline $0.5-0.25$ & 100.00 & - & - & - & - & - & - & - & - & - & - & - & - & - & - & - & - & - & - & - & - & - & - \\
\hline 1-0.5 & 100.00 & - & - & - & - & - & - & - & - & - & - & - & - & - & - & - & - & - & - & - & - & - & - \\
\hline $2-1$ & \begin{tabular}{|l|}
99.35 \\
\end{tabular} & - & - & 0.65 & - & - & - & - & - & - & - & - & - & - & - & - & - & - & - & - & - & - & - \\
\hline 3-2 & 100.00 & - & - & - & - & - & - & - & - & - & - & - & - & - & - & - & - & - & - & - & - & - & - \\
\hline $5-3$ & 100.00 & - & - & - & - & - & - & - & - & - & - & - & - & - & - & - & - & - & - & - & - & - & - \\
\hline Hole 60.0 & $-4-1,145$ & & & & & & & & & & & & & & & & & & & & & & \\
\hline $0.25-0.1$ & 94.66 & 0.66 & 2.00 & - & - & - & - & 2.68 & - & - & - & - & - & - & - & - & - & - & - & - & - & - & - \\
\hline $0.5-0.25$ & \begin{tabular}{|l|}
98.33 \\
\end{tabular} & 0.33 & 1.00 & - & - & - & - & 0.34 & - & - & - & - & - & - & - & - & - & - & - & - & - & - & - \\
\hline $1-0.5$ & 100.00 & - & - & - & - & - & - & - & - & - & - & - & - & - & - & - & - & - & - & - & - & - & - \\
\hline $2-1$ & 100.00 & - & - & - & - & - & - & - & - & - & - & - & - & - & - & - & - & - & - & - & - & - & - \\
\hline $3-2$ & 100.00 & - & - & - & - & - & - & - & - & - & - & - & - & - & - & - & - & - & - & - & - & - & - \\
\hline $5-3$ & 100.00 & - & - & - & - & - & - & - & - & - & - & - & - & - & - & - & - & - & - & - & - & - & - \\
\hline $7-5$ & 100.00 & - & - & - & - & - & - & - & - & - & - & - & - & - & - & - & - & - & - & - & - & - & - \\
\hline Hole 60.0 & $-8-1,145$ & & & & & & & & & & & & & & & & & & & & & & \\
\hline $0.25-0.1$ & 71.32 & 0.24 & 11.42 & 1.28 & - & - & 15.74 & - & - & - & - & - & - & - & - & - & - & - & - & - & - & - & - \\
\hline $0.5-0.25$ & 94.58 & - & 1.08 & 0.09 & - & - & 4.25 & - & - & - & - & - & - & - & - & - & - & - & - & - & - & - & - \\
\hline $1-0.5$ & 99.00 & - & - & - & - & - & 1.00 & - & - & - & - & - & - & - & - & - & - & - & - & - & - & - & - \\
\hline $2-1$ & 99.67 & - & - & - & - & - & 0.33 & - & - & - & - & - & - & - & - & - & - & - & - & - & - & - & - \\
\hline $3-2$ & 100.00 & - & - & - & - & - & - & - & - & - & - & - & - & - & - & - & - & - & - & - & - & - & - \\
\hline
\end{tabular}


TABLE 1 - Continued

\begin{tabular}{|c|c|c|c|c|c|c|c|c|c|c|c|c|c|c|c|c|c|c|c|c|c|c|c|}
\hline \multirow[b]{2}{*}{ 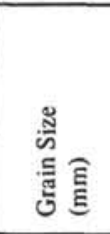 } & \multirow[b]{2}{*}{ 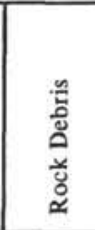 } & \multicolumn{2}{|c|}{ Volc. Glass } & \multirow[b]{2}{*}{ 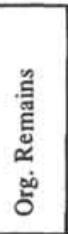 } & \multirow[b]{2}{*}{ 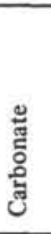 } & \multirow[b]{2}{*}{ है } & \multirow[b]{2}{*}{ 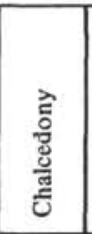 } & \multirow[b]{2}{*}{ 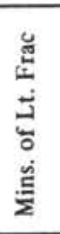 } & \multirow[b]{2}{*}{$\overrightarrow{\underline{\tilde{z}}}$} & \multirow[b]{2}{*}{ 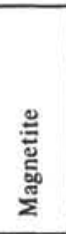 } & \multirow[b]{2}{*}{ 䓞 } & \multirow[b]{2}{*}{$\begin{array}{l}\stackrel{\mathscr{E}}{\rightleftarrows} \\
\text { E }\end{array}$} & \multirow[b]{2}{*}{ 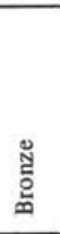 } & \multirow[b]{2}{*}{ 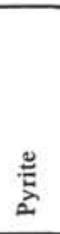 } & \multirow[b]{2}{*}{$\frac{\grave{0}}{0}$} & \multirow[b]{2}{*}{ 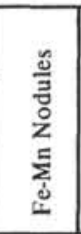 } & \multirow[b]{2}{*}{ 总 } & \multirow[b]{2}{*}{ 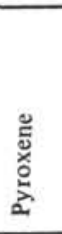 } & \multirow[b]{2}{*}{$\begin{array}{l}\text { 릉 } \\
\text { है } \\
\text { है }\end{array}$} & \multirow[b]{2}{*}{ 음 } & \multirow[b]{2}{*}{$\stackrel{\dddot{\Xi}}{\Sigma}$} & \multirow[b]{2}{*}{ 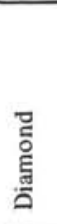 } & \multirow[b]{2}{*}{$\begin{array}{l}\stackrel{\mathscr{E}}{\Sigma} \\
\dot{\tilde{g}} \\
\underline{\Xi}\end{array}$} \\
\hline & & $\frac{\ddot{2}}{\frac{0}{0}}$ & 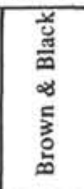 & & & & & & & & & & & & & & & & & & & & \\
\hline \multicolumn{24}{|c|}{ Hole $60.0-8-1,145 \mathrm{~cm}$ (Continued) } \\
\hline $5-3$ & 100.00 & - & - & - & - & - & - & - & - & - & - & - & - & - & - & - & - & - & - & - & - & - & - \\
\hline 7.5 & 100.00 & - & - & - & - & - & - & - & - & - & - & - & - & - & - & - & - & - & - & - & - & - & - \\
\hline \multicolumn{24}{|c|}{ Hole $60.0-9-5,16 \mathrm{~cm}$} \\
\hline $0.25-0.1$ & 60.80 & 2.09 & 6.15 & 0.23 & - & - & 20.02 & - & 0.30 & 1.26 & - & - & - & - & 0.06 & 9.09 & - & - & - & - & - & - & - \\
\hline $0.5-0.25$ & 73.66 & 10.13 & 10.66 & 0.17 & - & - & 5.37 & - & - & - & - & - & - & - & - & 0.01 & - & - & - & - & - & - & - \\
\hline $1-0.5$ & 97.67 & 1.00 & 1.34 & - & - & - & - & - & - & - & - & - & - & - & - & - & - & - & - & - & - & - & - \\
\hline $2 \cdot 1$ & 100.00 & - & - & - & - & - & - & - & - & - & - & - & - & - & - & - & - & - & - & - & - & - & - \\
\hline $3-2$ & 100.00 & - & - & - & - & - & - & - & - & - & - & - & - & - & - & - & - & - & - & - & - & - & - \\
\hline $5-3$ & 100.00 & - & - & - & - & - & - & - & - & - & - & - & - & - & - & - & - & - & - & - & - & - & - \\
\hline $7-5$ & 100.00 & - & - & - & - & - & - & - & - & - & - & - & - & - & - & - & - & - & - & - & - & - & - \\
\hline $10-7$ & 100.00 & - & - & - & - & - & - & - & - & - & - & - & - & - & - & - & - & - & - & - & - & - & - \\
\hline$>10$ & 100.00 & - & - & - & - & - & - & - & - & - & - & - & - & - & - & - & - & - & - & - & - & - & - \\
\hline
\end{tabular}

${ }^{2}$ Negligible traces of manganese, garnet (almondine), olivine, staurolite, barite and indeterminable ore minerals were found in some of these samples. 
TABLE 2A

Mineralogical Analysis of Heavy Fraction in Size Range 0.1 - $0.01 \mathrm{~mm}$

\begin{tabular}{|c|c|c|c|c|c|c|c|c|c|c|c|c|c|c|c|c|c|c|c|c|c|c|c|c|c|c|c|c|c|c|c|c|}
\hline $\begin{array}{c}\text { Sample } \\
\text { Designationa }\end{array}$ & 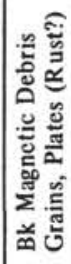 & 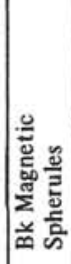 & 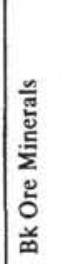 & 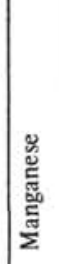 & 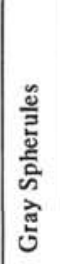 & $\tilde{\tilde{u}}$ & 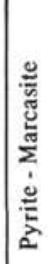 & 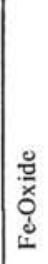 & 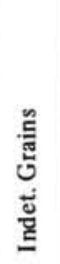 & Ð & 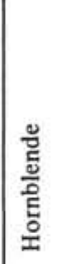 & 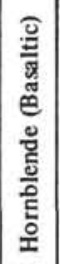 & 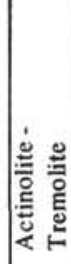 & 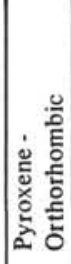 & 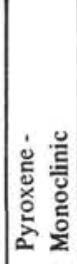 & 蒿 & 蒿 & 竞 & 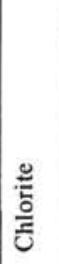 & 产 & 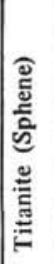 & ह & 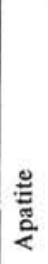 & 节 & E & 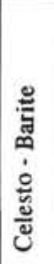 & 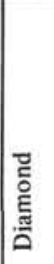 & 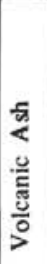 & 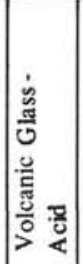 & 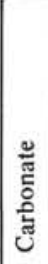 & 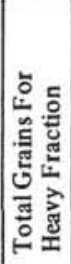 & 差 \\
\hline $\begin{array}{l}44.0-1-1,145 \\
\text { after acid }\end{array}$ & 55 & & & & & & & & & & & & & & & & & & & & & & & & & & & & & & 55 & \\
\hline $\begin{array}{l}44.0-1-2,145 \\
\text { after acid }\end{array}$ & 44 & & & & & & & & & & & & & & & & & & & & & & & & & & & & & & 44 & \\
\hline $\begin{array}{l}44.0-1-3,50 \\
\text { after acid }\end{array}$ & & & & & & & & & & & 1 & & & & & & & & & 1 & & & & & & & & & & & & \\
\hline $\begin{array}{l}44.0-2-\mathrm{CC} \\
\text { after acid }\end{array}$ & 29 & 2 & 4 & & & & & & 2 & 5 & 2 & & & 1 & 2 & & 5 & & 1 & & 1 & & & & & 1 & 1 & & & 1 & 57 & \\
\hline $44.0-2-1,145$ & & & & & & & & & & & & & & & & & & & & & & & & & & & & & & & & \\
\hline $\begin{array}{l}44.0-2-2,145 \\
\text { after acid } \\
(5 \%)\end{array}$ & & & & & & & & & & & & & & & & & & & & & & & & & & & & & & & & \\
\hline $\begin{array}{l}44.0-2-3,145 \\
44.0-2-4,145\end{array}$ & 15 & & & & & & & & & & & & & 1 & & & & & & & & & & & & & & & & & 16 & \\
\hline $\begin{array}{l}44.0-2-5 \\
\text { after acid }\end{array}$ & & & & & & & & & & & & & & & & & & & & & & & & & & & & & & & & \\
\hline $44.0-2-6,145$ & 15 & & & & & & & & & & & & & & & & & & & & & & & & & & & & & & 15 & \\
\hline $\begin{array}{l}\text { 44.0-3-CC } \\
\text { after acid } \\
(5 \%)\end{array}$ & & & & & 3 & & & & 5 & & 1 & & & & 1 & & & & & & & & & & & & $\begin{array}{r}10 \\
1\end{array}$ & & & & 446 & $427 \mathrm{~A}$ \\
\hline $\begin{array}{l}44.0-3-2,145 \\
\text { after acid } \\
44.0-3-3,145\end{array}$ & 6 & & & & . & & & & & & & & & & & & & & & & & & & & & & & & & & 6 & \\
\hline $\begin{array}{l}44.0-3-4,145 \\
\text { after acid }\end{array}$ & & & & & & & & & & & & & & & & & & & & & & & & & & & & & & & & \\
\hline $\begin{array}{l}44.0-3-5,145 \\
\text { after acid } \\
(5 \%)\end{array}$ & 9 & & & & 88 & & & & & & & & & & & & & & & & & & & & & & $\begin{array}{r}18 \\
3\end{array}$ & & & & 113 & $2^{\mathrm{A}}$ \\
\hline
\end{tabular}


TABLE 2A - Continued

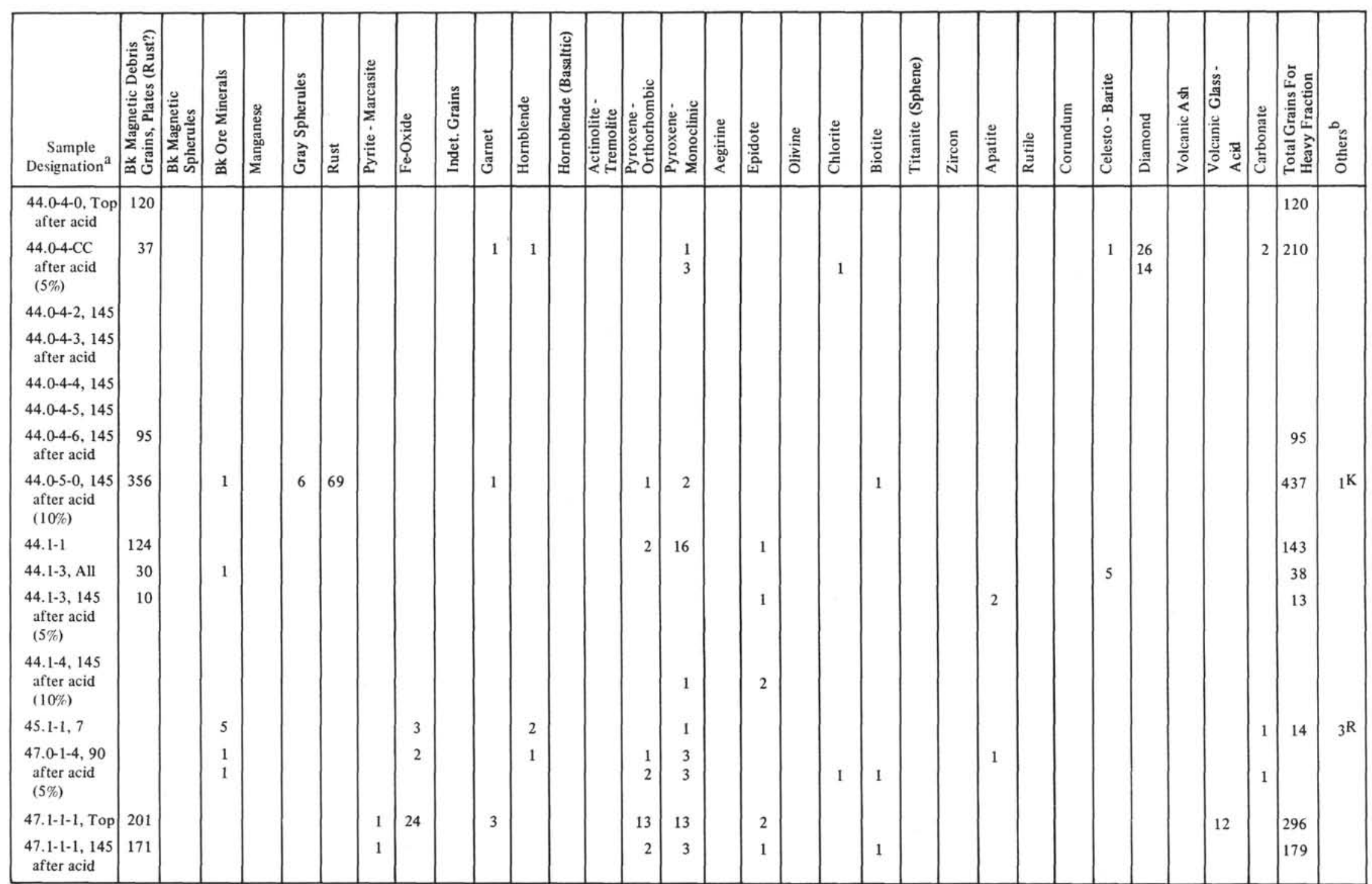


TABLE 2A - Continued

\begin{tabular}{|c|c|c|c|c|c|c|c|c|c|c|c|c|c|c|c|c|c|c|c|c|c|c|c|c|c|c|c|c|c|c|c|c|}
\hline $\begin{array}{c}\text { Sample } \\
\text { Designation }^{\mathrm{a}}\end{array}$ & 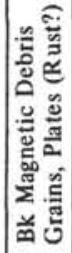 & 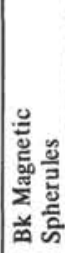 & 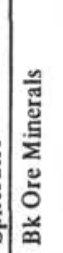 & 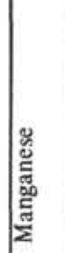 & 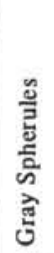 & $\begin{array}{l}\overrightarrow{\tilde{u}} \\
\overrightarrow{\tilde{\alpha}}\end{array}$ & 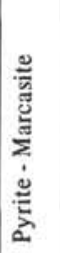 & 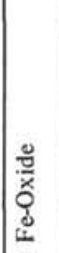 & 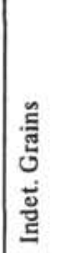 & 氖 & 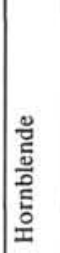 & 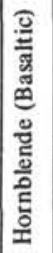 & 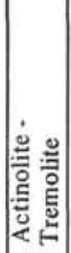 & 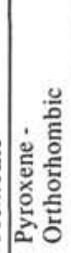 & 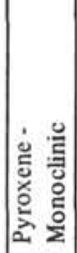 & 㗊 & 产 & $\stackrel{\mathscr{E}}{\stackrel{\Xi}{2}}$ & 起 & 总 & 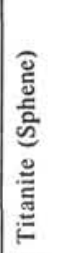 & 气. & 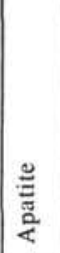 & $\stackrel{\underline{2}}{\underline{3}}$ & 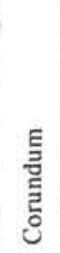 & 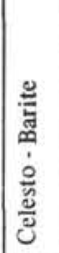 & 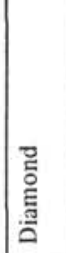 & 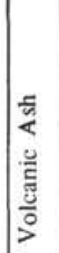 & 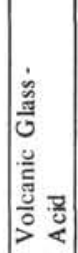 & 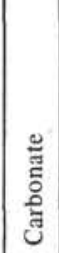 & 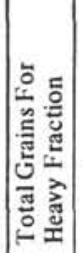 & 总 \\
\hline $47.2-1-2,23$ & 77 & & & & & & & 30 & & 2 & 9 & & & 30 & 71 & & & & & & & & & & & & & 38 & & & 257 & \\
\hline $\begin{array}{l}47.2-3-\mathrm{CC} \\
\text { after acid } \\
(5 \%)\end{array}$ & 12 & 1 & & & & & & & 2 & & 2 & & & 2 & 3 & & & & 1 & 1 & & & & & & & $\begin{array}{l}6 \\
1\end{array}$ & & & 1 & 534 & \\
\hline $47.2-4-\mathrm{CC}$ & 19 & 2 & & & 2 & & 11 & 1 & & 1 & & & & 3 & 2 & & & & & & & & & & & & 3 & & & & 44 & \\
\hline 47.2-8-6-CC & 167 & & & & 1 & & & 21 & & 3 & 2 & & & & & & 1 & & & & & & & & & & 2 & & 2 & & 199 & \\
\hline $47.2-9-\mathrm{CC}$ & 261 & & & & & & & 21 & 1 & & & & & & 1 & & & & 1 & & & 1 & & & 1 & & & & & & 287 & \\
\hline 47.2-9-CC & & & & & & & & & & & & & & & & & & & & & & & & & & & & & & & & \\
\hline $\begin{array}{l}47.2-9-1,150 \\
\text { after acid } \\
(5 \%)\end{array}$ & & & & & & & & & & & & & & & & & & & & & & & & & & & & & & & & $495^{\mathrm{A}}$ \\
\hline $47.2-9-4,0$ & & & & & & & & & & & & & & & & & & & & & & & & & & & & & & & & \\
\hline $\begin{array}{l}47.2-9-5,150 \\
\text { after acid } \\
(5 \%)\end{array}$ & & & & & & & & & & & & & & & & & & & & & & & & & & & & & & & & \\
\hline $\begin{array}{r}47.2-9-6,0 \\
\text { after acid }\end{array}$ & 25 & & & & & 37 & & & & & & & & & & & & & & & & & & & 9 & & & & & & 71 & \\
\hline $47.2-10-\mathrm{CC}$ & 68 & & & & & & 2 & 14 & & & & & & & 1 & & 3 & & & & & & & & & & 1 & & & & 82 & \\
\hline $\begin{array}{l}\text { 47.2-10, Top } \\
\text { after acid } \\
(10 \%)\end{array}$ & 150 & 6 & & & 40 & & & & & & & & & & & & & & & & & & & & $\begin{array}{r}11 \\
5\end{array}$ & & & & & & 211 & \\
\hline $47.2-11-\mathrm{CC}$ & 50 & & & & & & & & & & & & & & 4 & & & & 1 & & & & & & & & 3 & & & & 56 & \\
\hline $47.2-11-3,145$ & & 1 & & 1 & & 9 & & & & & & & & & & & & & & & & & & & 4 & & & & & & 15 & \\
\hline $\begin{array}{l}47.2-11-4,145 \\
\text { after acid }\end{array}$ & & & & & & & & & & & & & & & & & & & & & & & & & & & & & & & & \\
\hline $\begin{array}{c}47.2-11-6,145 \\
\text { after acid }\end{array}$ & & & & & & & & & & & & & & & & & & & & & & & & & & & & & & & & \\
\hline $47.2-12-\mathrm{CC}$ & 261 & & & & & & & & & 1 & & & & 1 & 1 & & 1 & & & & & & & & 1 & & 2 & & & 1 & 268 & $1^{A}$ \\
\hline
\end{tabular}


TABLE 2A - Continued

\begin{tabular}{|c|c|c|c|c|c|c|c|c|c|c|c|c|c|c|c|c|c|c|c|c|c|c|c|c|c|c|c|c|c|c|c|c|}
\hline $\begin{array}{c}\text { Sample } \\
\text { Designation }^{\mathrm{a}}\end{array}$ & 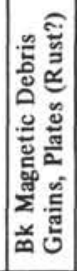 & 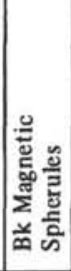 & 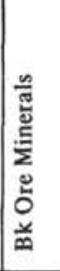 & 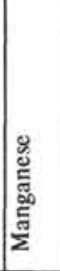 & 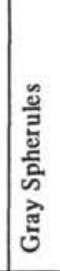 & $\overrightarrow{\underline{n}}$ & 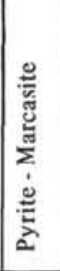 & $\begin{array}{l}\stackrel{0}{x} \\
0 \\
\vdots \\
\vdots \\
\end{array}$ & 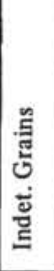 & 总 & 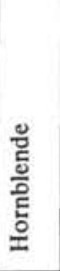 & 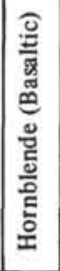 & 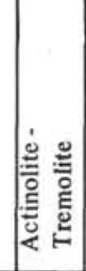 & 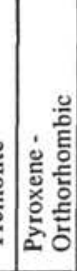 & \begin{tabular}{|cc}
0 \\
$\vdots$
\end{tabular} & 产 & 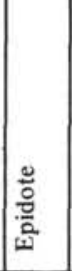 & $\frac{\mathscr{E}}{2}$ & 䒕 & 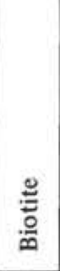 & 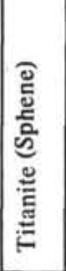 & 节 & 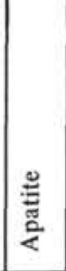 & $\stackrel{\varrho}{\check{\Xi}}$ & 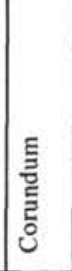 & 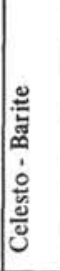 & 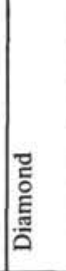 & 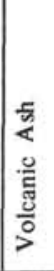 & 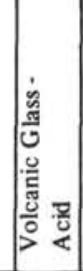 & 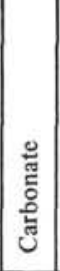 & 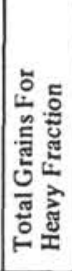 & $\begin{array}{l}0_{0} \\
\stackrel{0}{0} \\
0\end{array}$ \\
\hline $\begin{array}{l}47.2-12-1,145 \\
\text { after acid } \\
(5 \%)\end{array}$ & & & & & & & & & & & & & & & & & & & & & & & & & & & $\begin{array}{l}1 \\
2\end{array}$ & & & & & \\
\hline $\begin{array}{l}\text { 47.2-13-CC } \\
\text { after acid } \\
(10 \%)\end{array}$ & 11 & & & & & & & & & & & & & & 1 & & & & & & & & & & & & 2 & & & & 12 & \\
\hline $\begin{array}{l}47.2-13-5 \\
47.2-14-C C \\
\text { after acid }\end{array}$ & 103 & & & & & & & 2 & & & & & & & & & & & & & & & & & & & & & & & 105 & \\
\hline $\begin{array}{l}47.2-14-1,145 \\
\text { after acid } \\
(5 \%)\end{array}$ & & & & & & & & & & & & & & & & & & & & & & & & & 1 & & & & & & & \\
\hline $\begin{array}{l}48.2-1-C C \\
\text { after acid }\end{array}$ & 42 & & & & & & & 3 & & & & & 1 & 1 & & & & & 1 & & & & & & & & & & & & 48 & \\
\hline $\begin{array}{l}48.2-1-1,28 \\
\text { after acid } \\
(5 \%)\end{array}$ & & & & & & & & & & & & & & 2 & 3 & & & & & & & & & & 2 & & & & & & 7 & \\
\hline $48.2-2-\mathrm{CC}$ & 12 & & & & & & & & & & & & & & 1 & & & & & & & & & & & & & & & & 13 & \\
\hline $\begin{array}{l}48.2-2-1,145 \\
\text { after acid }\end{array}$ & & & & & & & & & & & & & & & & & & & & & & & & & & & & & & & & \\
\hline $49.0-1-1,11$ & & & 11 & & & & & 14 & & & 3 & & & 20 & 40 & & 1 & & & & & 1 & & & & & & & & & 138 & $4{ }_{1}^{R}$ \\
\hline $49.0-1-2,16$ & & & 55 & 3 & 124 & & & 42 & & & 6 & 1 & 1 & 51 & 194 & & & & & 1 & & 2 & 1 & & & 1 & & & & & 481 & \\
\hline $49.0-1-4,6$ & 114 & & & & & & & & & & 19 & & & 55 & 137 & & & & & & & & & & & & & 86 & & & 411 & \\
\hline $49.0-1-5,70$ & 22 & & 20 & & & & & 14 & & & & & & 2 & 2 & & & & & & & & & & & & & & & & 60 & \\
\hline $\begin{array}{c}49.0-2-1,70 \\
\text { after acid }\end{array}$ & & & & 83 & & & & 1 & & & & & & 2 & 2 & & & & & & & & & & & & 1 & & & & 89 & \\
\hline $49.1-1-1,77$ & 50 & & & 3 & & & & 37 & 48 & & 8 & & & 42 & 209 & & 1 & 1 & & & & & & & & 1 & & & & & 400 & \\
\hline $\begin{array}{l}49.1-1-2,124 \\
49.1-1-4,17\end{array}$ & & 1 & 13 & 22 & & & & 10 & & & 3 & & & 4 & 9 & & & & & 2 & & & & & & 2 & & & & & 66 & \\
\hline $\begin{array}{l}49.1-1-5,38 \\
49 \cdot 1-1-5.60\end{array}$ & 50 & & & & & & & & & & & & & & & & 1 & & & & & & & & & 3 & & & & & 54 & \\
\hline
\end{tabular}


TABLE 2A - Continued

\begin{tabular}{|c|c|c|c|c|c|c|c|c|c|c|c|c|c|c|c|c|c|c|c|c|c|c|c|c|c|c|c|c|c|c|c|c|}
\hline $\begin{array}{c}\text { Sample } \\
\text { Designation }^{\mathrm{a}}\end{array}$ & 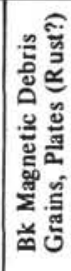 & 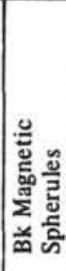 & 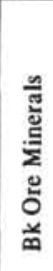 & 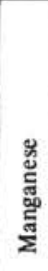 & 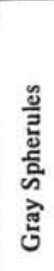 & $\stackrel{\vec{w}}{\simeq}$ & 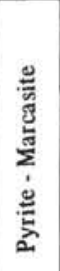 & 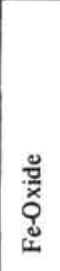 & 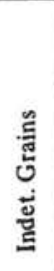 & 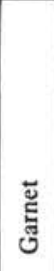 & 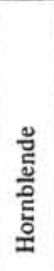 & 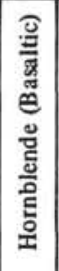 & 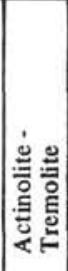 & 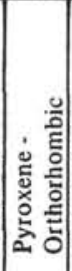 & 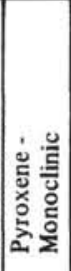 & 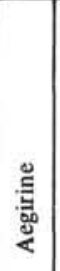 & 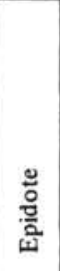 & 产 & 产 & 䒕 & 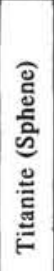 & 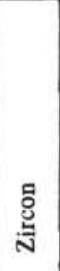 & 苋 & 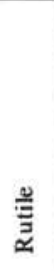 & 豆 & 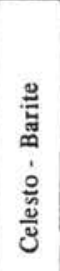 & 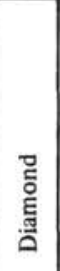 & 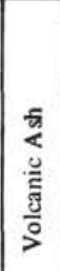 & 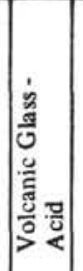 & 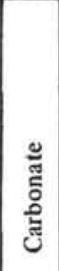 & 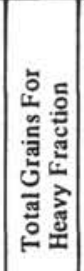 & 芯 \\
\hline $50.0-1-2,135$ & 109 & & & & & & & 151 & & & 1 & & & 2 & 4 & & 1 & & & & & & & & 3 & 20 & & & & & 298 & \\
\hline $\begin{array}{c}50.0-2-2,150 \\
\text { after acid }\end{array}$ & 88 & & & 11 & & & & 108 & & & & & & 1 & 7 & & 1 & & & & & & & & 1 & 14 & & & & & 231 & \\
\hline $50.0-2-3,150$ & 9 & & & 11 & 2 & & 5 & & & & & & & & 1 & & & & & & & & & & 2 & 26 & & & & & 56 & \\
\hline $50.0-2-4,150$ & 15 & & 3 & 7 & 38 & & & 3 & & & & & & & & & & & & & & & & & 2 & 10 & & & & & 78 & \\
\hline $50.0-2-6,150$ & 20 & & & & & & & & & & & & & & & & & & & & & & & & & & & & & & 20 & \\
\hline $50.1-1-1,62$ & & & 17 & & & & & 7 & & & 4 & 1 & & 43 & 102 & & & 1 & & 1 & & & 2 & & & & & & & & 250 & $73^{R}$ \\
\hline $50.1-1-2,15$ & & & 33 & & & & & 4 & & 1 & 29 & 1 & & 45 & 56 & & & 1 & & 2 & & 6 & 3 & & & & & & & & 252 & $71^{R}$ \\
\hline $50.1-1-4,17$ & & & 38 & & & & & 7 & & & 24 & 2 & & 41 & 86 & & 2 & 1 & & 3 & & 1 & 2 & 2 & & & & & & & 294 & $81^{R}$ \\
\hline $50.1-1-6,16$ & & & 62 & 34 & 15 & & & 25 & & & 28 & 3 & 1 & 23 & 55 & & & 1 & & 1 & & & 2 & & & & & 103 & & & 353 & \\
\hline $50.1-2-3,4$ & & & 20 & & 46 & & & 26 & & & 2 & & & 38 & 97 & & 1 & & 1 & 1 & & & & & & & & 125 & & & 367 & \\
\hline $50.1-2-4,12$ & & & 32 & & 36 & & & 28 & & & 54 & & & 22 & 84 & & 1 & 1 & & & & 1 & & & & 1 & & 62 & & & 322 & \\
\hline $50.1-2-4,50$ & 9 & & & & & & & & 3 & & 1 & & & & 6 & & 1 & & & & & & & & & & & & & & 20 & \\
\hline $50.1-3-1,145$ & & & & & & & & . & & & & & & & & & & & & & & & & & & & & & & & & \\
\hline $50.1-3-1,11$ & & & & & & & & & & & & & & & 4 & & & & & & & & & & & & & & & 1 & & \\
\hline $50.1-3-2,76$ & 13 & & & & & & & & & & 1 & & & 1 & 2 & & & & & & & & & & & & & 5 & & & 22 & \\
\hline $50.1-3-4,16$ & & & 3 & & & & & 5 & & & 2 & & & 9 & 58 & & 3 & & 1 & 1 & & & & & & & & & & & 91 & $11^{R}$ \\
\hline $50.1-3-6,16$ & & & 20 & & & & & 40 & & & 4 & & 1 & 2 & 7 & & 2 & & & 1 & & 1 & 2 & 1 & & 2 & & & & & 87 & ${ }_{4} R$ \\
\hline $51.0-1-C C$ & 200 & 1 & 4 & 2 & & & & & & & & & & & 4 & & & & & & & & & & 1 & & & & & 1 & 213 & \\
\hline $51.0-2-\mathrm{CC}$ & 500 & 2 & & & & & & & & & & & & 1 & 2 & 1 & & & 1 & & & & & & & & & & & & 507 & \\
\hline $51.1-1-2,16$ & & & 39 & 3 & & & & 16 & & & 7 & & & 42 & 116 & & & & & & & & 1 & & & & & 39 & & & 263 & \\
\hline $51.1-1 \cdot 4,30$ & 10 & & 42 & & 2 & & & & & & & & & 26 & 41 & & 2 & 1 & & 1 & & & & & & & & 48 & & 15 & 187 & \\
\hline $51.1-1-6,16$ & & & 54 & 2 & 12 & & & 30 & & & 40 & & & 35 & 79 & & & 1 & & 2 & & & & & & & & 30 & & & 385 & \\
\hline
\end{tabular}


TABLE 2A - Continued

\begin{tabular}{|c|c|c|c|c|c|c|c|c|c|c|c|c|c|c|c|c|c|c|c|c|c|c|c|c|c|c|c|c|c|c|c|c|}
\hline $\begin{array}{c}\text { Sample } \\
\text { Designation }^{\text {a }}\end{array}$ & 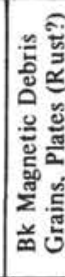 & 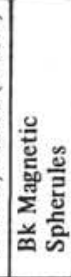 & 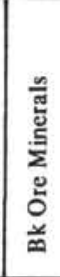 & 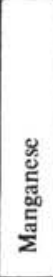 & 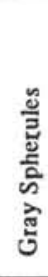 & $\tilde{\tilde{\Sigma}}$ & 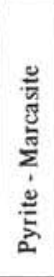 & 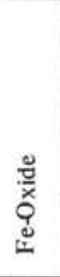 & 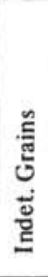 & 导 & 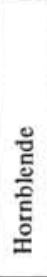 & 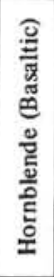 & 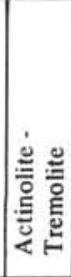 & 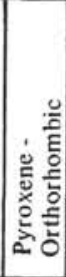 & 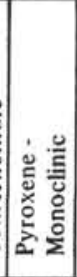 & 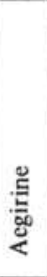 & $\begin{array}{l}\text { ․․ } \\
\stackrel{0}{0}\end{array}$ & $\frac{\stackrel{0}{2}}{3}$ & $\frac{\stackrel{\Xi}{ }}{\frac{0}{E}}$ & 气ั & 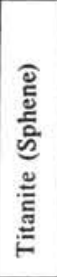 & 总 & $\begin{array}{l}\stackrel{g}{\underline{\sigma}} \\
\stackrel{\alpha}{<}\end{array}$ & 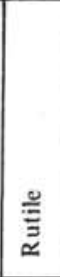 & 言 & 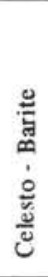 & 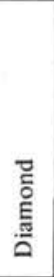 & $\begin{array}{l}\frac{5}{5} \\
\frac{0}{5} \\
\frac{0}{0} \\
\frac{0}{0}\end{array}$ & 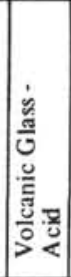 & 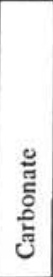 & 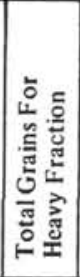 & 芒 \\
\hline $52.0-1-2,30$ & 14 & & 65 & & & & & & 14 & 1 & 1 & & & 82 & 171 & & & & & & & & 1 & & & & & 5 & & & 356 & \\
\hline $52.0-1-4,8$ & & & 66 & 3 & & & & 25 & & & 8 & & & 31 & 125 & & 3 & & & 3 & & & 1 & & & & & 22 & & & 287 & \\
\hline $52.0-1-6,9$ & & & 92 & & 32 & & & 27 & & & 1 & & & 43 & 187 & & 3 & & & & & & 3 & & & & & & & & 388 & \\
\hline $52.0-2-\mathrm{CC}$ & 4 & & 10 & & & & & 2 & 4 & & 1 & & & 5 & 9 & & & & 1 & & & & & & & & & & & & 35 & \\
\hline $52.0-1-3.7$ & & & 45 & & 30 & & & 37 & & & 4 & & & 19 & 142 & & 3 & & & & & & & & & & & 42 & & & 322 & \\
\hline $52.0-2-6,145$ & 5 & & 8 & & & & & & 2 & & 7 & & & 3 & 25 & & 2 & & 1 & 1 & & & & & & & & & & & 53 & \\
\hline $52.0-3-1,16$ & & & 132 & 7 & 25 & & & 47 & & & 3 & 2 & & 16 & 93 & & 3 & & & & & & 2 & & & & & & & & 330 & \\
\hline $52.0-3-3,16$ & & & 103 & & 44 & & & 71 & & & 2 & & 3 & 13 & 146 & & 3 & & & & & & 2 & & & 10 & & & & & 397 & \\
\hline $52.0-4-4,6$ & & & 99 & 1 & 30 & & & 89 & & & 4 & 1 & 3 & 2 & 17 & & 10 & & & & & & & & & & & & & & 256 & \\
\hline $52.0-4-6,18$ & & & 121 & 2 & 47 & & & 69 & & & 5 & & & 2 & 3 & & 42 & & 2 & & & & 1 & & & & & & & & 294 & \\
\hline $52.0-5-1,127$ & & & 98 & 3 & 50 & & & 81 & & & 4 & & & 2 & 11 & & 27 & & & & & & 2 & & & & & & & & 279 & \\
\hline $52.0-5-5,18$ & & & 84 & & 11 & & & 55 & & & 2 & & & 3 & 1 & & 25 & & & & & & 2 & & & & & & & & 183 & \\
\hline $52.0-6-\mathrm{CC}$ & 4 & & 12 & & & & & & & & & & & & 5 & & & & & & & & & & 1 & & & & & & 22 & \\
\hline $52.0-7-\mathrm{CC}$ & 250 & & & & 3 & & & & & & & & & & 1 & & & & 1 & & & 1 & & & & & & & & & 255 & \\
\hline $52.0-7-2,37$ & & & 4 & 28 & 2 & & & 2 & & & & & & & 1 & & & & 1 & 1 & & & & & & & & & & & 39 & \\
\hline $52.0-8-1,145$ & 31 & & & & & & & & & 2 & & & & 1 & 2 & 1 & 1 & & & & & & & & & & & & & 1 & 39 & \\
\hline $52.0-8-2,145$ & 131 & & & & & & & & 6 & & 5 & & & 7 & 8 & 1 & 4 & & & & 1 & & 1 & & 1 & & & & & & 165 & \\
\hline $52.0-8-3,40$ & 25 & & & & & & & & & & 2 & & & & & & & & & & & & & & & & & & & & 27 & \\
\hline $52.0-8-5,145$ & 92 & & & & & & & & & & 1 & & & 1 & & & 1 & & & & & & & & & & & & & & 95 & \\
\hline $52.0-8-5,145$ & & & 128 & & 12 & & & 44 & & & 1 & & & 2 & & & & & & & & & & & & & & & & & 187 & \\
\hline $52.0-9-\mathrm{CC}$ & 66 & & & & & & & 5 & & 1 & & & & 1 & 1 & & & & & & 1 & & & & & & & & & & 77 & \\
\hline $52.0-10-\mathrm{CC}$ & 19 & & & & & & & 6 & & & & & & 1 & 6 & & & & & & & & & & & & & 39 & & & 71 & \\
\hline $53.0-1-3,17$ & & & 62 & & 9 & & & 12 & & & & & & 60 & 159 & & 2 & & & & & & 6 & & & 1 & & & & & 311 & \\
\hline
\end{tabular}


TABLE 2A - Continued

\begin{tabular}{|c|c|c|c|c|c|c|c|c|c|c|c|c|c|c|c|c|c|c|c|c|c|c|c|c|c|c|c|c|c|c|c|c|}
\hline $\begin{array}{c}\text { Sample } \\
\text { Designation }\end{array}$ & 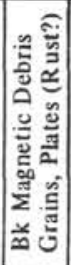 & 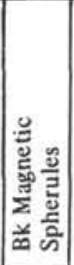 & 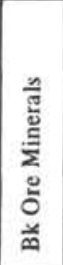 & 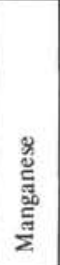 & 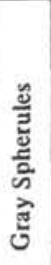 & $\stackrel{\vec{n}}{\ddot{z}}$ & 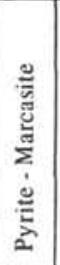 & 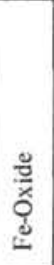 & 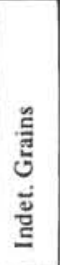 & 总 & 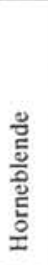 & 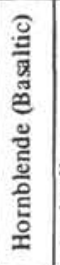 & 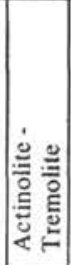 & 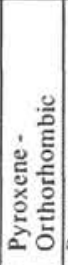 & 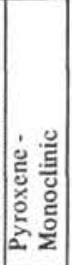 & 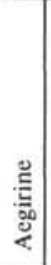 & 울 & $\stackrel{\stackrel{\Xi}{\Xi}}{\stackrel{\Xi}{0}}$ & 总 & 芯 & 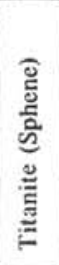 & 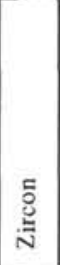 & 营 & 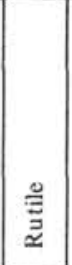 & 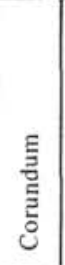 & 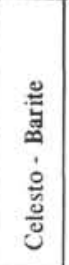 & 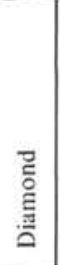 & 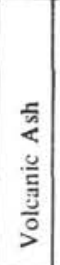 & 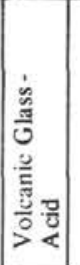 & 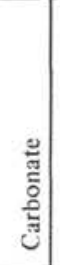 & 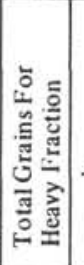 & 号 \\
\hline $\begin{array}{l}53.0-2-\mathrm{CC} \\
\text { after acid }\end{array}$ & 76 & & 156 & & & & & & 7 & 1 & & & & 9 & 16 & & 1 & & & & & & & & & 2 & 1 & & 19 & & 228 & \\
\hline $53.0-4-2$ & & 92 & & & & & & 26 & 64 & & 3 & & 1 & 90 & 190 & & 1 & 1 & & & & & & & & & & & & & 468 & \\
\hline $\begin{array}{l}53.0-6-\mathrm{CC} \\
\text { after acid }\end{array}$ & & & 170 & & & & & 5 & & & & & & & & & & & & & & & & & & & & & & 7 & 180 & \\
\hline $53.1-2-2,145$ & 58 & & & & & & & 16 & 34 & & 12 & 1 & & 95 & 145 & & 4 & 2 & & & & & 2 & & & & & & & & 369 & \\
\hline $53.1-3-1,150$ & 118 & & & & & & & 10 & 49 & 4 & 3 & & & 70 & 133 & 2 & 2 & & & & & & & & & & & & & & 391 & \\
\hline $53.1-3-2,40$ & 23 & & & & & & & 20 & 50 & & 1 & & & 43 & 132 & & 1 & & & & & & & & & & & & & & 270 & \\
\hline $53.2-4-\mathrm{CC}$ & & & & & & & & & & & & & & & & & & & & & & & & & & & & & & & & \\
\hline $53.2-5-\mathrm{CC}$ & & & & & & & & & & & & & & & & & & & & & & & & & & & & & & & & \\
\hline $54.0-1-1,5$ & & & 60 & & & & & 6 & & 2 & 1 & & & 74 & 148 & & 3 & 1 & & & & & 1 & 1 & & & & 32 & & & 335 & \\
\hline $54.0-1-2,7$ & & & & & & & & & & & & & & & & & & & & & & & & & & & & & & & & \\
\hline $54.0-3-1,140$ & & & 36 & & & & & 10 & & & so & & & 41 & 83 & & 1 & 2 & & & & & 1 & & 1 & 1 & & 17 & & & 243 & \\
\hline $54.0-6-1,145$ & & & & & & & & & & & & & & & & & & & & & & & & & & & & & & & & \\
\hline $\begin{array}{l}55.0-3-4,145 \\
\text { after acid }\end{array}$ & 6 & & & & & & & & & & & & & & & & & & & & & & & & & & & & & & 6 & \\
\hline $\begin{array}{l}55.0-9 \text {, Top } \\
\text { after acid }(5 \%)\end{array}$ & 16 & & & & & & & & & & 1 & & & & 1 & & & & & & & & & & & & & & & & 18 & \\
\hline $\begin{array}{l}55.0-13-3,40 \\
\text { after acid }\end{array}$ & & & & & & & & & & & & & & & & & & & & & & & & & & & & & & & & \\
\hline $55.2-2-3, \mathrm{CC}$ & & & & & & & & & & & & & & & & & & & & & & & & & & & & & & & & \\
\hline $\begin{array}{l}55.2-3, \text { Top } \\
57.1-3-1,17\end{array}$ & 250 & 20 & & & & & & & & & & & & 2 & 43 & & 1 & & & & & 1 & & & 1 & 1 & 2 & & & & 321 & \\
\hline $\begin{array}{l}57.1-4-4,145 \\
\text { after acid }(5 \%)\end{array}$ & 1 & & 1 & & & & & & & & & & & & 7 & & & & & & & & & & & & & & & & 20 & \\
\hline
\end{tabular}


TABLE 2A - Continued

\begin{tabular}{|c|c|c|c|c|c|c|c|c|c|c|c|c|c|c|c|c|c|c|c|c|c|c|c|c|c|c|c|c|c|c|c|c|}
\hline $\begin{array}{c}\text { Sample } \\
\text { Designation }^{\mathrm{a}}\end{array}$ & 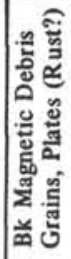 & 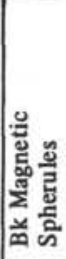 & 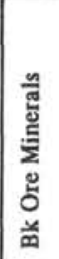 & 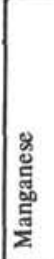 & 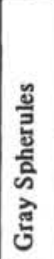 & $\overrightarrow{\underline{z}}$ & 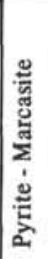 & 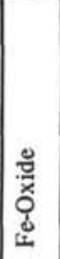 & 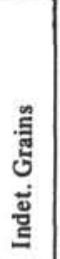 & E్ & 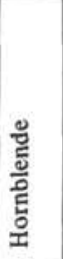 & 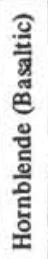 & 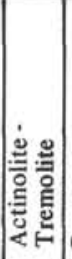 & 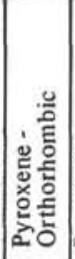 & 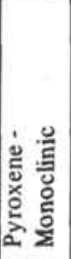 & 咅 & 产 & 趇 & 莒 & $\frac{9}{0}$ & 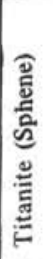 & 总 & 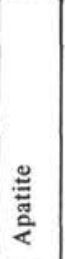 & $\stackrel{\dddot{\Xi}}{3}$ & 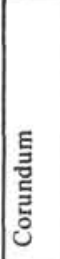 & 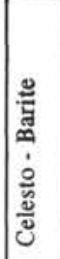 & 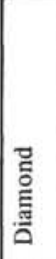 & 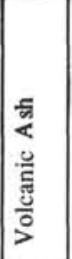 & 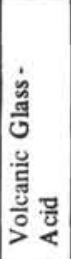 & 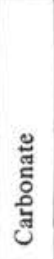 & 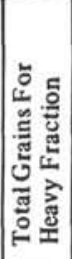 & 。 \\
\hline $58.2-1-5,0$ & 47 & & & & & & & & 92 & & 1 & & & & 259 & & 1 & & & & & & & & & & & & & 2 & 402 & \\
\hline $58.2-1-6,95$ & & 32 & & & & & & 6 & 27 & & & & & & 12 & & & & 2 & & & & 23 & & & & & & & & 81 & $2 \mathrm{~A}$ \\
\hline $59.1-3-\mathrm{CC}$ & & & & & & & & & & & & & & & & & & & 1 & & & & & & & & & & & & & \\
\hline $59.2-1-\mathrm{CC}$ & 75 & & & & & & & & & & & & & 5 & 34 & & & & & & & & & & & & & & & & 114 & \\
\hline $59.2-2-\mathrm{CC}$ & 42 & & & & & & & 108 & & & 1 & & & 5 & 14 & & 2 & & & & & 1 & & & 1 & & & & & & 174 & \\
\hline 59.2-6, Тор BU & & & & & & & & & & & & & & & & & & & & & & & & & & & & & & & & \\
\hline $60.0-1-\mathrm{CC}$ & 80 & & & & & & & & & & 1 & & & 12 & 15 & & 1 & & & & & & & & & & & & & & 109 & \\
\hline $60.0-2-\mathrm{CC}$ & 68 & & & & & & & 6 & 8 & 4 & 4 & 2 & & 119 & 131 & & 1 & & & & & & 1 & 1 & & & & & & & 345 & \\
\hline $60.0-3-\mathrm{CC}$ & 196 & & & & & & & 5 & 9 & & 4 & & & 42 & 68 & & 1 & & & & & & & & & & & 48 & 21 & & 394 & \\
\hline $60.0-4-C C$ & 92 & & & & & & & 12 & & 1 & 1 & & & 33 & 63 & & 2 & & & & & & & 1 & & & & 55 & 41 & & 301 & \\
\hline $60.0-4-\mathrm{CC}$ & & 4 & 146 & & 5 & & & 9 & & 2 & 1 & & & 38 & 49 & & 1 & & 1 & & & & 1 & & & & & 18 & & & 274 & \\
\hline $60.0-4-2,145$ & & & & & & & & & & & & & & & & & & & 1 & & & & & & & & & & & & & \\
\hline $\begin{array}{l}\text { 60.0-5-CC } \\
\text { after acid }\end{array}$ & & 1 & 38 & & & & & 9 & & 1 & 1 & & & 16 & 31 & & 1 & & 1 & 1 & & & 2 & & & & & 36 & & 1 & 138 & $1^{A}$ \\
\hline $60.0-7-1,81.5$ & & & 6 & & & & & & & & & & & & & & & & & & & & & & & & & & & & 6 & \\
\hline
\end{tabular}

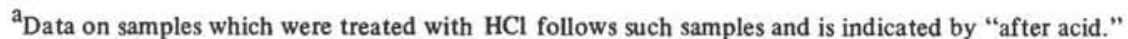

${ }^{\mathrm{b}}$ Other minerals: $\mathrm{A}=$ Alloy $; \mathrm{K}=$ Kyanite $; \mathrm{L}=$ Limonite $; \mathrm{M}=$ Muscovite $\mathrm{O}=\mathrm{Opal}$ debris $; \mathrm{R}=\mathrm{Rock}$ debris $\mathrm{P}=\mathrm{Palagonite} ; \mathrm{V}=$ Volcanic glass basic 
TABLE 2B

Mineralogical Analysis of Light Fraction in Size Range $0.1-0.01 \mathrm{~mm}$

\begin{tabular}{|c|c|c|c|c|c|c|c|c|c|c|c|c|c|c|c|c|c|c|c|c|c|c|c|c|c|c|c|c|c|c|}
\hline $\begin{array}{c}\text { Sample } \\
\text { Designation }^{\mathrm{a}}\end{array}$ & 音 & 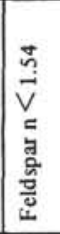 & 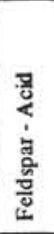 & 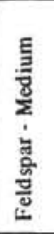 & 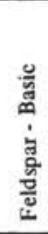 & 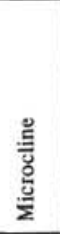 & 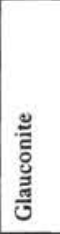 & 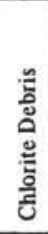 & 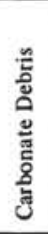 & 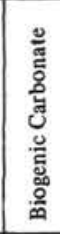 & 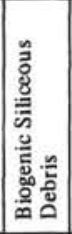 & 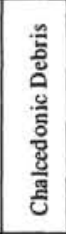 & 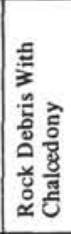 & 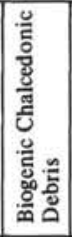 & 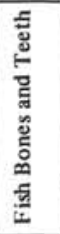 & 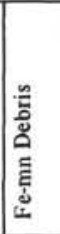 & 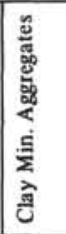 & $\begin{array}{l}\stackrel{\Xi}{0} \\
\text { N }\end{array}$ & $\begin{array}{l}\frac{5}{5} \\
\frac{a}{0} \\
\frac{0}{\tilde{E}} \\
\frac{\sqrt[3]{3}}{0} \\
>\end{array}$ & 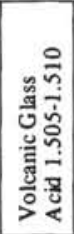 & 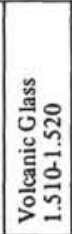 & 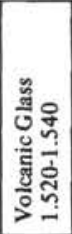 & 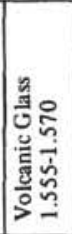 & 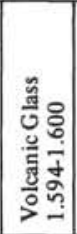 & 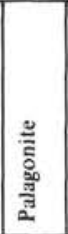 & 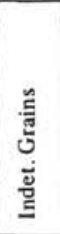 & 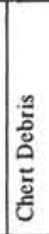 & 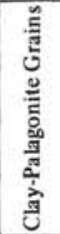 & 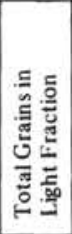 & \\
\hline $\begin{array}{l}44.0-1-1,145 \\
\text { after acid }\end{array}$ & 1 & & & & & & & & & 264 & & & & & 3 & & & & & & & & & & & & & & 268 & \\
\hline $\begin{array}{l}44.0-1-2,145 \\
\text { after acid }\end{array}$ & & & & & & & & & & 231 & & & & & 3 & & 7 & & & & & & & & & & & & $\begin{array}{r}234 \\
7\end{array}$ & \\
\hline $\begin{array}{l}44.0-1-3,50 \\
\text { after acid (5\%) }\end{array}$ & 19 & & 2 & $\begin{array}{l}1 \\
4\end{array}$ & & & & & & 327 & & 5 & 2 & 1 & 2 & & & & & $\begin{array}{l}1 \\
3\end{array}$ & & 1 & & & 1 & & & & $\begin{array}{r}331 \\
48\end{array}$ & $8^{\mathrm{R}}$ \\
\hline $\begin{array}{l}\text { 44.0-2-CC } \\
\text { after acid (5\%) }\end{array}$ & $\begin{array}{r}54 \\
180\end{array}$ & $\begin{array}{l}2 \\
4\end{array}$ & $\begin{array}{r}18 \\
106\end{array}$ & 1 & & $\begin{array}{l}1 \\
1\end{array}$ & 1 & & & 293 & & & 2 & 2 & 15 & & & & & $\begin{array}{l}1 \\
3\end{array}$ & & & & & & $\begin{array}{r}1 \\
22\end{array}$ & & & $\begin{array}{l}392 \\
326\end{array}$ & $4 \mathrm{M}$ \\
\hline $44.0-2-1,145$ & 1 & 1 & & & & & & & & 425 & & & & & 78 & & & & & 1 & & & & & & & & & 506 & \\
\hline $\begin{array}{l}40.2-2,145 \\
\text { after acid (5\%) }\end{array}$ & $\begin{array}{l}2 \\
7\end{array}$ & 1 & 3 & $\begin{array}{l}2 \\
2\end{array}$ & & & & & & 320 & & & & & 20 & & & & & $\begin{array}{l}2 \\
8\end{array}$ & & & 1 & & & & & & $\begin{array}{r}346 \\
27\end{array}$ & ${ }_{3} R$ \\
\hline $44.0-2-3,145$ & 1 & & 1 & & & & & & & 387 & & & & & 24 & & 2 & & & & 2 & & & & & & & & 417 & \\
\hline $44.0-2-4,145$ & & & & & & & & & & 251 & & & & & 11 & & & & & & & 1 & & & & & & & 263 & \\
\hline $\begin{array}{l}44.0-2-5 \\
\text { after acid }\end{array}$ & & & & & & & & & & 246 & & & & & 14 & & & & & & & & & & & & & & 260 & \\
\hline $44.0-2-6,145$ & & & & & & & & & & 252 & & & & & 32 & & & & & & & 1 & & & & & & & 285 & \\
\hline $\begin{array}{l}\text { 44.0-3-CC } \\
\text { after acid }(5 \%)\end{array}$ & $\begin{array}{l}1 \\
2\end{array}$ & 1 & & & & & & & & 273 & & $\begin{array}{l}156 \\
228\end{array}$ & 48 & 3 & 3 & & & & & $\begin{array}{l}1 \\
2\end{array}$ & & & & & & 4 & & & $\begin{array}{l}437 \\
292\end{array}$ & $\begin{array}{l}1^{\mathrm{M}} \\
1^{\mathrm{M}}\end{array}$ \\
\hline $\begin{array}{l}44.0-3-2,145 \\
\text { after acid (5\%) }\end{array}$ & $\begin{array}{r}2 \\
10\end{array}$ & 1 & $\begin{array}{l}1 \\
2\end{array}$ & 1 & & & & & & 337 & & $\begin{array}{r}2 \\
45\end{array}$ & & & 9 & & 1 & & & $\begin{array}{l}1 \\
4\end{array}$ & & & 1 & & & & & & $\begin{array}{r}354 \\
67\end{array}$ & $2 \mathrm{O}$ \\
\hline $44.0-3-3,145$ & 2 & & & & & & & & & 250 & & & & 1 & 19 & & & & & & 1 & & & & & & & & 273 & \\
\hline $\begin{array}{l}44.0-3-4,145 \\
\text { after acid }(10 \%)\end{array}$ & $\begin{array}{l}1 \\
5\end{array}$ & & 1 & $\begin{array}{l}1 \\
3\end{array}$ & & & & & & 322 & & $\begin{array}{r}2 \\
15\end{array}$ & & & 25 & $\begin{array}{l}1 \\
4\end{array}$ & & & & $\begin{array}{l}1 \\
6\end{array}$ & & 1 & & & & & & & $\begin{array}{r}354 \\
41\end{array}$ & $5^{R}$ \\
\hline $\begin{array}{l}44.0-3-5,145 \\
\text { after acid (5\%) }\end{array}$ & $\begin{array}{l}1 \\
2\end{array}$ & & 1 & & & & & & 4 & 102 & & $\begin{array}{l}110 \\
100\end{array}$ & $\begin{array}{r}115 \\
99\end{array}$ & & 6 & & 1 & & & $\begin{array}{l}1 \\
2\end{array}$ & & & & & & $\begin{array}{l}12 \\
12\end{array}$ & & & $\begin{array}{l}359 \\
227\end{array}$ & $\begin{array}{l}5 \mathrm{O} \\
8 \mathrm{O}\end{array}$ \\
\hline $\begin{array}{l}\text { 44.0-4-0, Top } \\
\text { after acid (10\%) }\end{array}$ & 12 & 1 & & 2 & & & & & & 275 & 3 & 191 & & & 5 & & & & & $\begin{array}{r}1 \\
10\end{array}$ & & 1 & $\begin{array}{l}1 \\
2\end{array}$ & & & & & & $\begin{array}{l}285 \\
226\end{array}$ & ${ }_{1} \mathrm{R}$ \\
\hline $\begin{array}{l}44.0-4-C C \\
\text { after acid }(5 \%)\end{array}$ & $\begin{array}{r}1 \\
24\end{array}$ & 1 & $\begin{array}{r}1 \\
10\end{array}$ & & & & & & & 361 & 1 & $\begin{array}{r}4 \\
150 \\
\end{array}$ & 60 & 1 & 7 & $\begin{array}{r}2 \\
18\end{array}$ & & & & & & 1 & & & 2 & $\begin{array}{r}4 \\
10 \\
\end{array}$ & & & $\begin{array}{l}382 \\
299 \\
\end{array}$ & $1^{\mathrm{M}}$ \\
\hline
\end{tabular}


TABLE 2B - Continued

\begin{tabular}{|c|c|c|c|c|c|c|c|c|c|c|c|c|c|c|c|c|c|c|c|c|c|c|c|c|c|c|c|c|c|c|}
\hline $\begin{array}{c}\text { Sample } \\
\text { Designation }^{\mathrm{a}}\end{array}$ & 駦 & 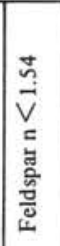 & 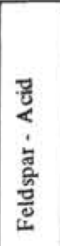 & 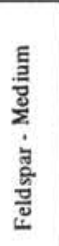 & 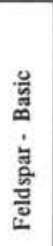 & 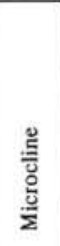 & 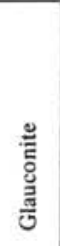 & 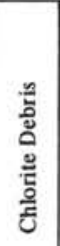 & 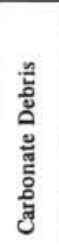 & 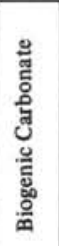 & 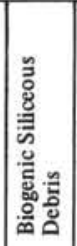 & 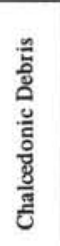 & 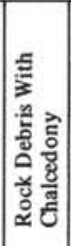 & 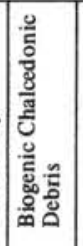 & 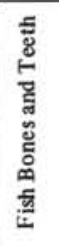 & 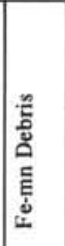 & 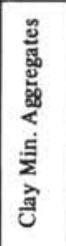 & 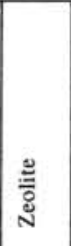 & 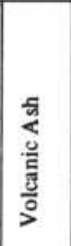 & 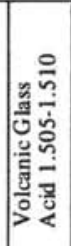 & 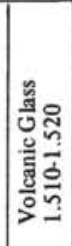 & 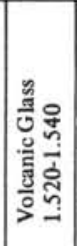 & 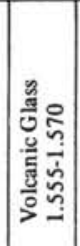 & 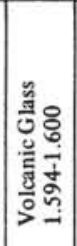 & $\begin{array}{l}\stackrel{\Xi}{E} \\
\text { 总 } \\
\text { है } \\
\text { है }\end{array}$ & 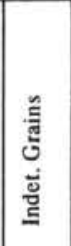 & 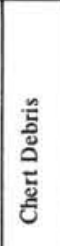 & 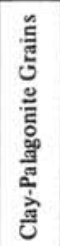 & 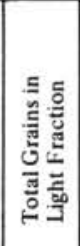 & 踝 \\
\hline $44.0-4-2,145$ & & & & & & & & & & 379 & & & & & 4 & & & & & & & & & & & & & & 383 & \\
\hline $44,0-4-3,145$ & & & & & & & & & & 333 & & & & & 2 & & & & & & & & & & & & & & 335 & \\
\hline $44.0-4-4,145$ & & & & & & & & & & 346 & & & & & 3 & & & & & & & & & & & & & & 349 & \\
\hline $44.0-4-5,145$ & 3 & & & & & 1 & & & & 252 & & & & & 45 & & & & & 1 & & & & & & & & & 302 & \\
\hline $\begin{array}{l}44.0-4-6,145 \\
\text { after acid }(5 \%)\end{array}$ & $\begin{array}{r}1 \\
14\end{array}$ & & 3 & $\begin{array}{l}1 \\
2\end{array}$ & & & & & & 288 & & $\begin{array}{l}2 \\
7\end{array}$ & 5 & & 5 & $\begin{array}{r}6 \\
32\end{array}$ & & 1 & & 1 & & & 1 & & & & & & $\begin{array}{r}304 \\
68\end{array}$ & ${ }_{2} \mathrm{R}$ \\
\hline $\begin{array}{l}44.0-5-0,145 \\
\text { after acid (10\%) }\end{array}$ & 5 & & & 1 & & & & & & 325 & & $\begin{array}{r}16 \\
283\end{array}$ & & & 2 & $\begin{array}{l}1 \\
3\end{array}$ & & & & 2 & & & & & & & & & $\begin{array}{l}348 \\
299\end{array}$ & $\begin{array}{l}{ }_{2}^{R} \\
{ }_{2} \mathrm{R}\end{array}$ \\
\hline $44.1-1$ & 2 & 1 & & 7 & & & & 1 & & 13 & 1 & 4 & & & 69 & 11 & 147 & 1 & & & 7 & 5 & & & 10 & & & & 282 & \\
\hline 44.1-3, All & & & & & & & & & & 328 & & & & & 3 & & & & & & & & & & & & & & 331 & \\
\hline $\begin{array}{l}44.1-3,145 \\
\text { after acid (5\%) }\end{array}$ & 19 & 2 & 8 & 3 & & & & & & 388 & 1 & $\begin{array}{r}2 \\
34\end{array}$ & & 3 & 2 & & 1 & 1 & & 2 & & 1 & & & 4 & 4 & & & $\begin{array}{r}393 \\
86\end{array}$ & ${ }_{3} \mathrm{R}$ \\
\hline $\begin{array}{l}44.1-4,145 \\
\text { after acid (10\%) }\end{array}$ & 9 & 4 & 7 & 4 & & & & & & 388 & 1 & 1 & & 2 & 5 & & & 1 & & $\begin{array}{l}1 \\
3\end{array}$ & & & & & 3 & 4 & & & $\begin{array}{r}339 \\
48\end{array}$ & ${ }_{6} \mathrm{R}$ \\
\hline $45.1-1.7$ & 2 & & 2 & 27 & & & & & 1 & 1 & & 1 & & 3 & 5 & 2 & 104 & & & 26 & & 1 & 12 & & 3 & & & & 234 & $1^{\mathrm{M}}$ \\
\hline $\begin{array}{l}47.0-1-4,90 \\
\text { after acid (5\%) }\end{array}$ & 1 & & 1 & $\begin{array}{l}2 \\
8\end{array}$ & & & & & 2 & 273 & $\begin{array}{l}6 \\
6\end{array}$ & 1 & & 2 & 2 & & 2 & & & $\begin{array}{r}69 \\
244\end{array}$ & & $\begin{array}{l}1 \\
4\end{array}$ & $\begin{array}{l}1 \\
1\end{array}$ & & 1 & & & & $\begin{array}{l}369 \\
311\end{array}$ & $\begin{array}{l}4{ }_{4}^{\mathrm{R}} \\
1^{\mathrm{M}}\end{array}$ \\
\hline 47.1-1-1, Top & & & & 13 & & & & & & 157 & 1 & & & & 4 & & 3 & & 7 & 12 & & & 2 & & 1 & & & & 201 & \\
\hline $\begin{array}{l}47.1-1-1,145 \\
\text { after acid }\end{array}$ & 1 & & & 1 & & & & & & 243 & & & & & 3 & & & 2 & 1 & 8 & & & & & & 1 & & & 261 & \\
\hline $47.2-1-2,23$ & & & & 2 & 23 & & & & & & 14 & & & 1 & & & 3 & & so & & 252 & 3 & 3 & & & & & & 350 & \\
\hline $\begin{array}{l}47.2-3-C C \\
\text { after acid }(5 \%)\end{array}$ & $\begin{array}{l}1 \\
7\end{array}$ & & 2 & $\begin{array}{r}3 \\
19\end{array}$ & & & 1 & & & 288 & $\begin{array}{l}7 \\
4\end{array}$ & $\begin{array}{l}2 \\
4\end{array}$ & & & 3 & & & 1 & & $\begin{array}{r}16 \\
188\end{array}$ & & $\begin{array}{l}1 \\
3\end{array}$ & $\begin{array}{l}1 \\
3\end{array}$ & & & & & & $\begin{array}{l}333 \\
334\end{array}$ & $\mid \begin{array}{c}{ }_{1}^{\mathrm{M}} \\
101^{\mathrm{R}}\end{array}$ \\
\hline $47: 2-4-C C$ & 1 & & & 1 & & & & & & 264 & 2 & & & & 5 & & & & & 3 & & & 1 & & & & & & 278 & \\
\hline $47.2-8-6-\mathrm{CC}$ & 20 & 4 & 5 & & & & & & & & 2 & 65 & & & & & & 8 & 7 & 16 & & 4 & 4 & & 4 & & & & 134 & \\
\hline 47.2-9-CC & 1 & & & & & & & 1 & & 187 & 2 & & & & 78 & & 3 & 5 & & & & & & & & & & & 275 & \\
\hline
\end{tabular}


TABLE 2B - Continued

\begin{tabular}{|c|c|c|c|c|c|c|c|c|c|c|c|c|c|c|c|c|c|c|c|c|c|c|c|c|c|c|c|c|c|c|}
\hline $\begin{array}{c}\text { Sample } \\
\text { Designation }^{\mathrm{a}}\end{array}$ & 影 & 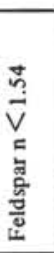 & 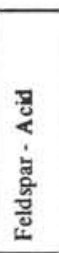 & 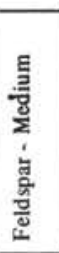 & 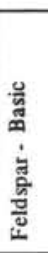 & 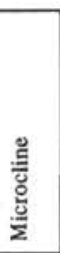 & 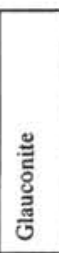 & 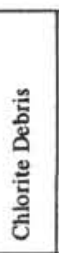 & 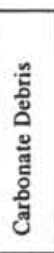 & 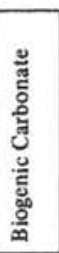 & 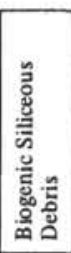 & 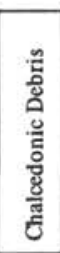 & 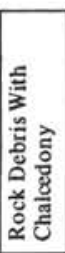 & 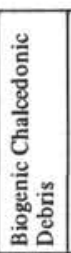 & 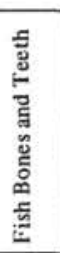 & 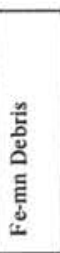 & 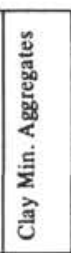 & 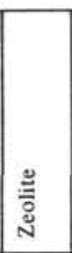 & 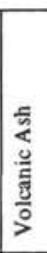 & 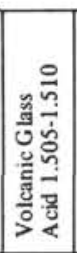 & 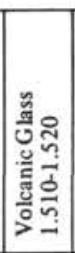 & 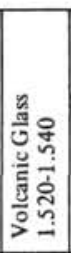 & 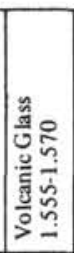 & 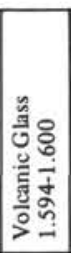 & 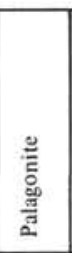 & 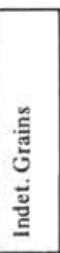 & 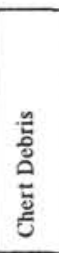 & 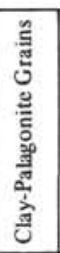 & 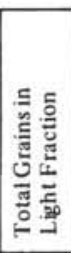 & 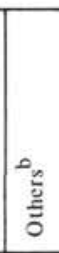 \\
\hline $47.2-9-\mathrm{CC}$ & & & & 3 & & & & 2 & & 3 & & 66 & & & 27 & & & 45 & & 3 & & 1 & 1 & & & 13 & & & 171 & \\
\hline $\begin{array}{l}47.2-9-1,150 \\
\text { after acid (5\%) }\end{array}$ & 2 & & 1 & $\begin{array}{l}1 \\
1\end{array}$ & & & & & & 360 & & & $\begin{array}{r}1 \\
15\end{array}$ & & 18 & 1 & & & & $\begin{array}{r}5 \\
17\end{array}$ & & 2 & 1 & & & & & & $\begin{array}{r}385 \\
44\end{array}$ & ${ }_{4} \mathrm{R}$ \\
\hline $47.2-9-4,0$ & 3 & 1 & & & & & & & & 348 & & & & & 77 & & 2 & & & & 1 & & & & & & & & 432 & \\
\hline $\begin{array}{l}47.2-9-5,150 \\
\text { after acid (5\%) }\end{array}$ & 1 & & & 1 & & & & & & 332 & & & $\begin{array}{l}10 \\
60\end{array}$ & & 33 & & & & & $\begin{array}{l}2 \\
2\end{array}$ & & & & & 3 & & & & $\begin{array}{r}377 \\
70\end{array}$ & $3^{R}$ \\
\hline $\begin{array}{l}47.2-9-6,0 \\
\text { after acid }\end{array}$ & 1 & & & 1 & & & & & & 524 & & & & & 100 & & & 25 & & & 1 & 3 & & & & 2 & & & 657 & \\
\hline $47.2-10-\mathrm{CC}$ & & & 1 & & & & & & & 304 & & & & & 18 & & & & & & & & & & & & & & 323 & \\
\hline $\begin{array}{l}47.2-10 \\
\text { after acid (10\%) }\end{array}$ & $\begin{array}{r}1 \\
14\end{array}$ & 2 & 2 & 6 & & 1 & & & & 351 & 1 & $\begin{array}{r}3 \\
49\end{array}$ & & 4 & 3 & & 1 & 5 & & 13 & & 1 & 2 & & 3 & & & & $\begin{array}{l}358 \\
119\end{array}$ & ${ }_{2} \mathrm{P}$ \\
\hline $47.2-11-\mathrm{CC}$ & 4 & 3 & 3 & 1 & & & & & & 306 & 1 & 19 & & & 16 & & & 1 & & & & & & & & 2 & & & 364 & ${ }_{2 \mathrm{R}}^{2 \mathrm{M}}$ \\
\hline $47.2-11-3,145$ & & & & & & & & & & 321 & & 1 & & & 1 & & & & & & & & & & & & & & 323 & \\
\hline $\begin{array}{l}47.2-11-4,145 \\
\text { after acid }\end{array}$ & & & 1 & & & & & & & 265 & & & & & 4 & 1 & 1 & 1 & & 1 & & & & & & & & & 274 & \\
\hline $\begin{array}{l}47.2-11-6,145 \\
\text { after acid }\end{array}$ & & & & & 1 & & & & & 295 & & 7 & & & 6 & 3 & 9 & & & & 7 & 1 & & 5 & 3 & & & & 337 & \\
\hline $47.2-12-\mathrm{CC}$ & 3 & 3 & 3 & 1 & & & & & & 222 & & 98 & & & 23 & & 1 & 1 & & 1 & & & 1 & & & & & & 362 & ${ }_{1}^{\mathrm{M}}$ \\
\hline $47.2-12-1,145$ & 3 & & & 1 & & & & & & 252 & & 113 & & & 16 & 1 & & & & 4 & & & 1 & & & & & & 435 & ${ }_{10}$ \\
\hline after acid (5\%) & 10 & & 1 & 3 & & & & & & & & 197 & & 1 & & 3 & & & & 12 & & & 5 & & & & & & 241 & $\begin{array}{l}1_{2}^{\mathrm{F}} \\
2^{\mathrm{O}} \\
2^{\mathrm{P}}\end{array}$ \\
\hline $47 \cdot 2-13-\mathrm{CC}$ & 1 & & & 1 & & & & & & 352 & 1 & 2 & & & 2 & & & 1 & & & & & & & & & & & 360 & \\
\hline after acid $(10 \%)$ & 25 & 2 & 7 & 2 & & & & & & & 3 & 138 & & & & & & $i$ & & 2 & & & & & & & & & 188 & $\begin{array}{l}4 \mathrm{O} \\
2^{\mathrm{R}}\end{array}$ \\
\hline $47.2-13-5$ & 11 & 8 & 13 & 4 & & 1 & & 2 & & & 2 & 111 & & 6 & 11 & 4 & & & & 2 & & & 1 & & 2 & 3 & & & 181 & \\
\hline
\end{tabular}


TABLE 2B - Continued

\begin{tabular}{|c|c|c|c|c|c|c|c|c|c|c|c|c|c|c|c|c|c|c|c|c|c|c|c|c|c|c|c|c|c|c|}
\hline $\begin{array}{c}\text { Sample } \\
\text { Designation }^{\text {a }}\end{array}$ & $\frac{v}{\mathrm{v}}$ & 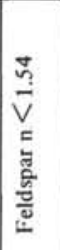 & 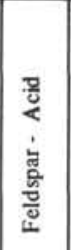 & 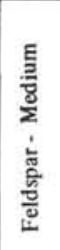 & 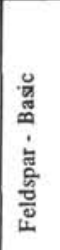 & 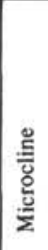 & 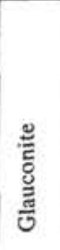 & 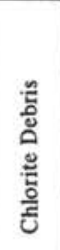 & 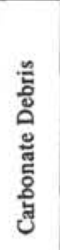 & 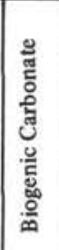 & 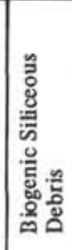 & 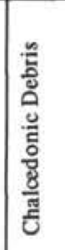 & 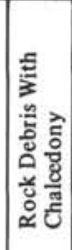 & 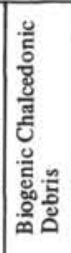 & 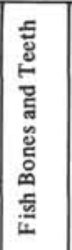 & 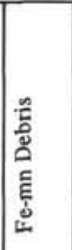 & 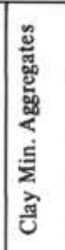 & 芩 & 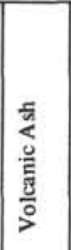 & 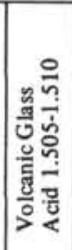 & 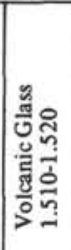 & 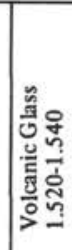 & 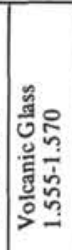 & 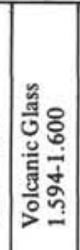 & $\begin{array}{l}\text { 离 } \\
\text { 品 } \\
\text { है }\end{array}$ & 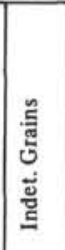 & 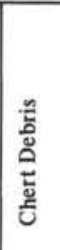 & 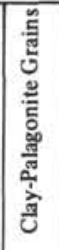 & 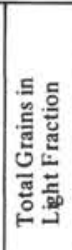 & 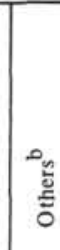 \\
\hline $\begin{array}{l}47.2-14-\mathrm{CC} \\
\text { after acid }\end{array}$ & & & 1 & & & & & & & 254 & & & & & 1 & & & & & & & & & & & & & & 256 & \\
\hline $\begin{array}{l}47.2-14-1,145 \\
\text { after acid (5\%) }\end{array}$ & 1 & & & & & & & & & 309 & 1 & $\begin{array}{r}2 \\
18\end{array}$ & & & 3 & & & & & $\begin{array}{r}4 \\
40\end{array}$ & & & 2 & & 1 & & & & $\begin{array}{r}319 \\
72\end{array}$ & $\begin{array}{l}1^{R} \\
1^{O}\end{array}$ \\
\hline $\begin{array}{l}48.2-1-\mathrm{CC} \\
\text { after acid }\end{array}$ & 1 & & 1 & 1 & & & & 1 & & 236 & 1 & & & & 4 & & & 4 & & 2 & & & 1 & & & & & & 252 & \\
\hline $\begin{array}{l}48.2-1-1,28 \\
\text { after acid }(5 \%)\end{array}$ & $\begin{array}{l}2 \\
1\end{array}$ & & & $\begin{array}{l}2 \\
4\end{array}$ & & & 1 & & 48 & 263 & $\begin{array}{l}4 \\
2\end{array}$ & 1 & & & 5 & & $\begin{array}{r}4 \\
10\end{array}$ & $\begin{array}{r}2 \\
24\end{array}$ & & & $\begin{array}{l}26 \\
31\end{array}$ & $\begin{array}{l}1 \\
1\end{array}$ & $\begin{array}{l}1 \\
3\end{array}$ & & $\begin{array}{l}2 \\
5\end{array}$ & & & & $\begin{array}{l}368 \\
179\end{array}$ & $\begin{array}{l}8 \mathrm{R} \\
36^{\mathrm{R}}\end{array}$ \\
\hline $48.2-2-\mathrm{CC}$ & 1 & & & & & & & & & 293 & & & & 1 & & & & & & & & & & & & & & & 295 & \\
\hline $\begin{array}{l}48.2-2-1,145 \\
\text { after acid }\end{array}$ & 1 & & & & & & & & & 388 & & 3 & & 2 & & & & & & & & & & & & & & & 294 & \\
\hline $49.0-1-1,11$ & 1 & & 3 & 23 & & & & & & 1 & 1 & 3 & & 2 & 29 & 20 & 63 & 36 & & 182 & & 9 & 3 & & & & & & 387 & $17 \mathrm{R}$ \\
\hline $49.0-1-2,16$ & & & 6 & & 17 & & & & & & & & & & 3 & & & & 30 & 244 & & 1 & & 1 & & & & & 302 & \\
\hline $49.0-1-4,6$ & 4 & 2 & 39 & 2 & & & & & & & 3 & & & & 3 & & & & 96 & 226 & & 2 & 4 & & & & & & 381 & \\
\hline $49.0-1-5,70$ & & 1 & & 3 & 37 & & & & & & & & & & 63 & 80 & 102 & 12 & & 72 & & 1 & & & & 11 & & & 382 & \\
\hline $\begin{array}{l}49.0-2-1,70 \\
\text { after acid }\end{array}$ & & & & & & & & 64 & & 94 & & & & 24 & 4 & 3 & 7 & 2 & & 11 & & & & & & & & & 209 & \\
\hline $49.1-1-1,77$ & 1 & & 1 & 1 & 30 & & & & & 1 & & & & 3 & & 9 & & & 60 & 229 & & 2 & & & 1 & & & & 338 & \\
\hline $49.1-1-2,124$ & & 1 & & & 2 & & & & & & & 1 & & & 98 & 15 & 3 & 210 & & 5 & & 2 & & & 1 & & & & 338 & \\
\hline $49.1-1-4,17$ & & & & & & & & & & & & & & 2 & 218 & 19 & 21 & 74 & & & & & & & & & & & 334 & \\
\hline $49.1-1-5,38$ & & & & & & & & & & 29 & & & & 159 & 28 & 47 & 7 & 11 & & 19 & & & & & & & & & 300 & \\
\hline $49.1-1-5,60$ & 1 & 2 & 5 & 8 & & & & & 1 & 7 & & 3 & & 14 & 57 & 43 & 36 & 104 & & & 3 & & 1 & & 2 & & & & 294 & ${ }_{7} \mathrm{R}$ \\
\hline $50.0-2-1,145$ & & & & 1 & & & & 3 & 1 & & & 148 & 6 & 64 & 6 & 11 & & 1 & & & & & 1 & & 4 & 10 & & & 256 & \\
\hline $\begin{array}{l}50.0-2-2,150 \\
\text { after acid }\end{array}$ & 1 & & 1 & 2 & & & 1 & 2 & & 124 & & $\begin{array}{l}130 \\
161\end{array}$ & & $\begin{array}{l}41 \\
62\end{array}$ & 7 & $\begin{array}{l}7 \\
3\end{array}$ & 8 & $\begin{array}{r}4 \\
10\end{array}$ & 1 & 2 & & 1 & 1 & & 4 & & & & $\begin{array}{l}312 \\
256\end{array}$ & \\
\hline $50.0-2-3,150$ & & & 1 & & & & & & & & & 147 & & 98 & & 9 & 10 & 1 & & & 1 & & & & 1 & 2 & & & 270 & \\
\hline
\end{tabular}


TABLE 2B - Continued

\begin{tabular}{|c|c|c|c|c|c|c|c|c|c|c|c|c|c|c|c|c|c|c|c|c|c|c|c|c|c|c|c|c|c|c|}
\hline $\begin{array}{c}\text { Sample } \\
\text { Designation }^{\mathrm{a}}\end{array}$ & 善 & 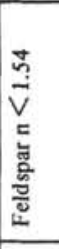 & 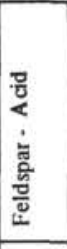 & 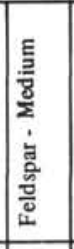 & 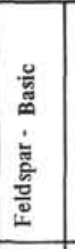 & 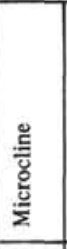 & 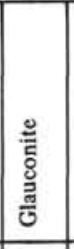 & 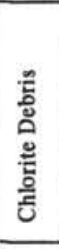 & 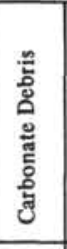 & 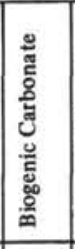 & 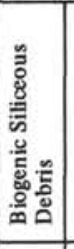 & 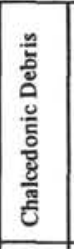 & 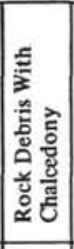 & 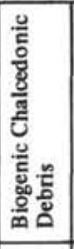 & 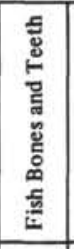 & 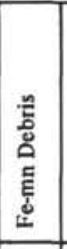 & 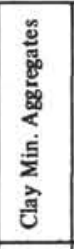 & $\begin{array}{l}\text { :ँّ } \\
\text { N. } \\
\end{array}$ & 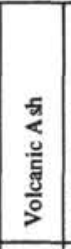 & 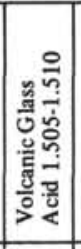 & 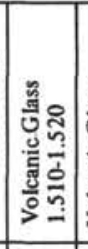 & 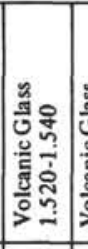 & 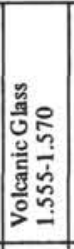 & 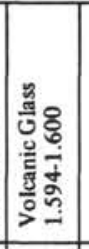 & 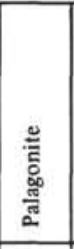 & 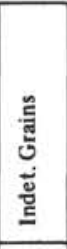 & 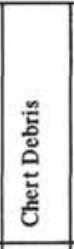 & 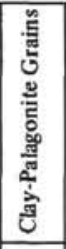 & 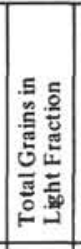 & 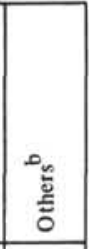 \\
\hline $50.0-2-4,150$ & 1 & & & & & & & & 35 & 13 & & 217 & & 110 & 9 & 4 & & 2 & & & & & & & 1 & 2 & & & 394 & \\
\hline $50.0-2-6,150$ & & & & 1 & & & & & 54 & 6 & & 193 & & 108 & 8 & 6 & & 1 & & & 1 & & & & 3 & & & & 389 & $8^{R}$ \\
\hline $50.1-1-1,1,62$ & & & & 6 & & & & & & & 125 & 1 & & 1 & 2 & 1 & 4 & 3 & & 95 & & 2 & 2 & & & & & & & $\begin{array}{l}1^{\mathrm{P}} \\
4{ }^{\mathrm{R}}\end{array}$ \\
\hline $50.1-1-2,15$ & 1 & & 3 & 5 & & & & & & & 31 & & & 1 & & & 2 & 1 & & 236 & & & 2 & & & & & & & $17 \mathrm{R}$ \\
\hline $50.1-1-4,17$ & 2 & & 1 & 11 & & & & & & 21 & 29 & & & & 4 & 6 & 15 & 1 & & 146 & & 2 & 1 & & & & & & & $\begin{array}{l}1^{\mathrm{P}} \\
66^{\mathrm{R}}\end{array}$ \\
\hline $50.1-1-6,16$ & 2 & 1 & 1 & & 19 & & & & & & 1 & & & 2 & & 8 & & & 83 & 164 & 10 & 4 & & & & & & & 296 & ${ }_{1} \mathrm{~V}$ \\
\hline $50.1-2 \cdot 3,4$ & 2 & & 3 & & 35 & & & & & & 2 & & & & 1 & & & & 123 & 208 & & 2 & & & & & & & 376 & \\
\hline $50.1 \cdot 2-4,12$ & 1 & & 4 & & 54 & & & & & 1 & 4 & & & & 3 & 10 & & & 86 & 188 & & 1 & & & 1 & & & & 356 & $3 \mathrm{v}$ \\
\hline $50.1-2-4,50$ & 1 & & 2 & 6 & & & & & 2 & 1 & & 1 & & 3 & 201 & 3 & 78 & & & & 6 & 2 & 6 & & 5 & & & & 332 & $15^{\mathrm{R}}$ \\
\hline $50.1-3-1,145$ & 4 & 1 & 4 & 53 & & 1 & & & & 1 & 2 & & & 12 & 18 & 56 & 118 & 1 & & 20 & & 1 & 1 & & 1 & 3 & & & 313 & $\begin{array}{l}1^{P} \\
15^{R}\end{array}$ \\
\hline $50.1-3-1,145$ & 5 & 3 & 9 & 39 & & & & & & 1 & 3 & 6 & 27 & 61 & 9 & 45 & 32 & 2 & & 19 & & 2 & 3 & & 1 & 5 & & & 305 & $28^{R}$ \\
\hline $50.1-3-2,76$ & 3 & & 3 & 7 & 27 & & & & & 1 & 5 & & & & 29 & 27 & 62 & & 30 & 85 & & 1 & 1 & & & & & & 281 & \\
\hline $50.1-3-4,16$ & 1 & & 2 & 21 & & & & & & & & 1 & & 7 & 11 & 29 & 116 & 7 & & 56 & & 7 & 3 & & & & & & 280 & $17 \mathrm{R}$ \\
\hline $50.1-3-6,16$ & 3 & 1 & 5 & 20 & & 1 & & & & & & 1 & & 9 & 27 & 20 & 133 & 138 & & 6 & & 1 & 1 & & & 9 & & & 383 & $\begin{array}{l}1^{\mathrm{P}} \\
{ }_{7 \mathrm{R}}\end{array}$ \\
\hline $51.0-1-\mathrm{CC}$ & & 1 & 2 & 4 & & 1 & 1 & & 2 & 5 & 2 & & & 7 & 233 & 21 & 33 & 4 & & & 31 & 3 & 5 & & 4 & & & & 372 & $13^{\mathrm{R}}$ \\
\hline $51.0-2-\mathrm{CC}$ & 1 & & 7 & 10 & & & & & 1 & 1 & 5 & 61 & & 50 & 96 & 21 & 14 & 1 & & & 1 & & & & 22 & 25 & & & 298 & \\
\hline $51 \cdot 1-1-2,16$ & 1 & & 3 & & 69 & & & & & & & 2 & & & 4 & 5 & & 7 & 113 & 37 & & 13 & & & 1 & & & & 255 & \\
\hline $51.1-1-4,30$ & 2 & & 1 & 62 & & & & & & 1 & & & & 4 & 3 & & 4 & & & & 36 & 2 & 12 & & 3 & & & & 306 & $175^{\mathrm{R}}$ \\
\hline $51 \cdot 1 \cdot 1-6,16$ & & 1 & 1 & & 27 & & & & & & 2 & & & 1 & & 2 & 2 & 2 & 36 & & 198 & 27 & & & & & & & 299 & \\
\hline $52.0-1-2,30$ & 9 & 1 & 5 & 50 & & & 2 & & 1 & 9 & & & & 23 & 2 & & 13 & 1 & & & 12 & & & & 9 & & & & 267 & $89 \mathrm{R}$ \\
\hline $52.0-1-4,8$ & 1 & 5 & 1 & & 37 & & & & & & & & & & & 17 & & & 47 & 147 & & 3 & & & & & & & 270 & $12 \mathrm{~L}$ \\
\hline $52.0-1-6,9$ & 1 & 4 & 5 & & 96 & & & & & & & & & & & 23 & & 1 & 73 & 79 & & 2 & & & 2 & & & & 286 & \\
\hline $52.0-2-\mathrm{CC}$ & 2 & & 5 & 165 & & & & & & 1 & & 2 & & 6 & & & 5 & & & & 4 & & & & 2 & 3 & & & 280 & $84 \mathrm{R}$ \\
\hline
\end{tabular}


TABLE 2B - Continued

\begin{tabular}{|c|c|c|c|c|c|c|c|c|c|c|c|c|c|c|c|c|c|c|c|c|c|c|c|c|c|c|c|c|c|c|}
\hline $\begin{array}{c}\text { Sample } \\
\text { Designation }\end{array}$ & 赔 & 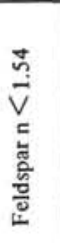 & 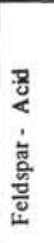 & 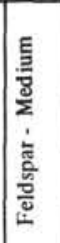 & 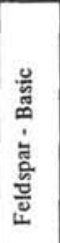 & 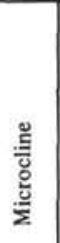 & 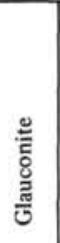 & 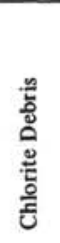 & 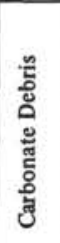 & 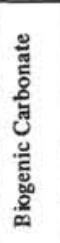 & 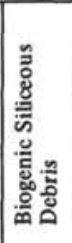 & 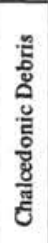 & 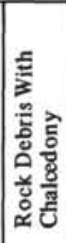 & 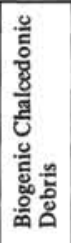 & 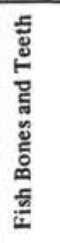 & 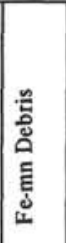 & 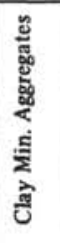 & 气 & 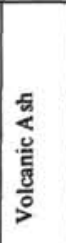 & 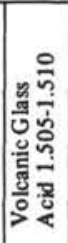 & 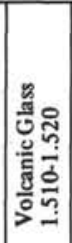 & 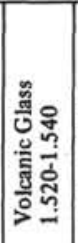 & 哭。 & 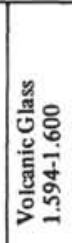 & 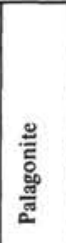 & 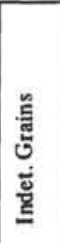 & 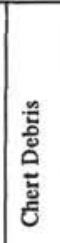 & 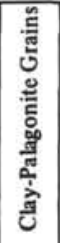 & 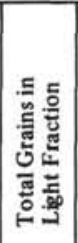 & 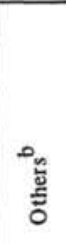 \\
\hline $52.0-2-3,7$ & 1 & 1 & 1 & & 174 & & & & & & 1 & & & & 1 & & 16 & & 56 & 49 & & 1 & & & & & & & 301 & \\
\hline $52.0-2-6,145$ & 5 & 1 & 6 & 96 & & 1 & 1 & & 1 & 1 & 1 & 2 & & 8 & 2 & 3 & 93 & & & & & 1 & 1 & & 3 & 15 & & & 279 & $37 \mathrm{R}$ \\
\hline $52.0-3-1,16$ & 2 & 1 & 6 & & 155 & & & & & & 5 & 2 & & & 2 & 11 & & 1 & 30 & 17 & & 2 & & & 1 & 8 & & & 258 & $15 \mathrm{~L}$ \\
\hline $52.0-3-3,16$ & & 3 & 2 & & 110 & & & & & & 2 & & & & & 2 & & 1 & 60 & & 26 & 14 & & 1 & 2 & 33 & & & 256 & \\
\hline $52.0-4-4,6$ & 2 & 6 & 5 & & 91 & & & & & & & & & 1 & 1 & 32 & 56 & 1 & 5 & & 1 & & & & & 55 & & & 256 & \\
\hline $52.0-4-6,18$ & 4 & 5 & 3 & & 115 & & & & & & & & & & 2 & 35 & 61 & & & 5 & & & & & & 46 & & & 287 & $\begin{array}{l}1^{\mathrm{M}} \\
1^{\mathrm{V}} \\
{ }^{2} \mathrm{~L}\end{array}$ \\
\hline $52.0-5 \cdot 1,127$ & 1 & 1 & 5 & & 63 & & & & & 2 & & 9 & & & 2 & 78 & 75 & 4 & & & 1 & & & & & & & & 244 & \\
\hline $52.0-5-5,18$ & 3 & 9 & 4 & & 38 & & & & & 1 & & & 3 & & 2 & 71 & 53 & & & 3 & & & & 1 & & 65 & & & 254 & ${ }_{1} \mathrm{M}$ \\
\hline $52.0-6-\mathrm{CC}$ & 3 & & 2 & 12 & & & & & & & & 3 & 13 & 1 & 5 & 2 & 67 & & & 4 & & & 3 & & 1 & & & & 121 & ${ }_{5} \mathrm{R}$ \\
\hline $52.0-7-\mathrm{CC}$ & 2 & & & 1 & & & 1 & & & & & & 120 & 245 & 2 & 1 & & 1 & & & 1 & 1 & & & & & & & 376 & \\
\hline $52.0-7-2,37$ & 1 & 1 & 1 & & & & & & & & & & & 7 & 202 & 19 & 19 & 5 & & & & & & & 3 & 3 & & & 269 & \\
\hline $52.0-8-1,145$ & 1 & 1 & 1 & 7 & & & & & & 2 & & & & 336 & 33 & 3 & & 2 & & & 2 & 3 & 1 & & 1 & & & & 394 & ${ }_{1} \mathrm{R}$ \\
\hline $52.0-8-2,145$ & & & & & & & & & & & & & 198 & 73 & 60 & 6 & 66 & & & & & & & & & & & & 403 & \\
\hline $52.0-8 \cdot 3,40$ & 1 & 1 & & & & & & & & & & & 176 & 46 & 35 & 1 & 10 & & & 1 & & & 1 & & 1 & & & & 273 & \\
\hline $52.0-8-5,145$ & 1 & & & & & & & 1 & & & & & 219 & 33 & 5 & 2 & 9 & & & & 2 & & & & & & & & 272 & \\
\hline $52.0-8-5,145$ & & & & & 1 & & & & & & & & 247 & & & 2 & 46 & & & & & & & & & & & & 296 & \\
\hline $52.0-9-\mathrm{CC}$ & & 2 & & & & & & & & & & & 118 & 34 & 182 & 8 & 8 & 1 & & & & & & & 1 & & & & 354 & \\
\hline $52.0-10-C C$ & 3 & & & 3 & & 1 & & & & & & & 48 & 26 & 32 & 14 & 11 & & 38 & & 11 & 8 & 4 & & 1 & & & & 200 & \\
\hline $53.0-1-3,17$ & & & & & 31 & & & & & & & & 3 & & & & & & 64 & 178 & & 15 & & 7 & & & & & 298 & \\
\hline $\begin{array}{l}53.0-2-\mathrm{CC} \\
\text { after acid }\end{array}$ & $\begin{array}{r}1 \\
10\end{array}$ & 1 & 5 & $\begin{array}{l}13 \\
51\end{array}$ & & 1 & & & & 2 & $\begin{array}{l}2 \\
1\end{array}$ & 1 & & & 3 & 3 & $\begin{array}{r}288 \\
23\end{array}$ & & & $\begin{array}{l}33 \\
56\end{array}$ & & 9 & $\begin{array}{l}10 \\
43\end{array}$ & & & & & & $\begin{array}{l}375 \\
329\end{array}$ & $\begin{array}{l}24^{\mathrm{R}} \\
2^{\mathrm{P}} \\
120^{\mathrm{R}}\end{array}$ \\
\hline $53.0-4-2$ & & & & & 19 & & 1 & & & & & & 24 & 2 & 14 & & 180 & 1 & 31 & & 2 & 3 & 5 & & & & & & 282 & \\
\hline $\begin{array}{l}53 \cdot 0-6-C C \\
\text { after ac id }\end{array}$ & $\begin{array}{l}1 \\
2\end{array}$ & & $\begin{array}{r}3 \\
80\end{array}$ & $\begin{array}{l}1 \\
4\end{array}$ & & & & & & 10 & & 1 & & 2 & & & & 4 & & $\begin{array}{l}3 \\
4\end{array}$ & & 1 & $\begin{array}{l}1 \\
3\end{array}$ & & 1 & 99 & & & $\begin{array}{l}343 \\
251\end{array}$ & $\begin{array}{l}1 \mathrm{M} \\
47^{\mathrm{R}}\end{array}$ \\
\hline
\end{tabular}


TABLE 2B - Continued

\begin{tabular}{|c|c|c|c|c|c|c|c|c|c|c|c|c|c|c|c|c|c|c|c|c|c|c|c|c|c|c|c|c|c|c|}
\hline $\begin{array}{c}\text { Sample } \\
\text { Designation }^{a}\end{array}$ & 롤 & 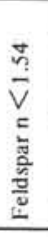 & 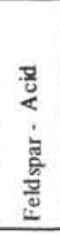 & 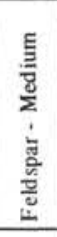 & 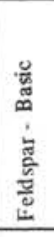 & 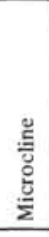 & 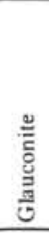 & 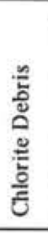 & 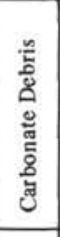 & 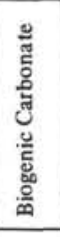 & 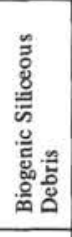 & 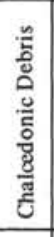 & 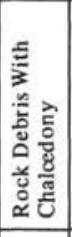 & 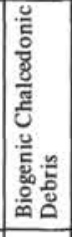 & 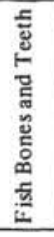 & 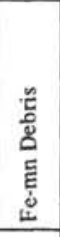 & 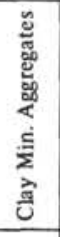 & 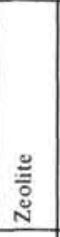 & 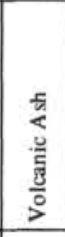 & 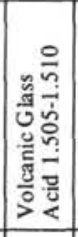 & 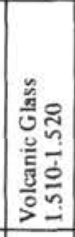 & 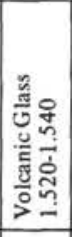 & 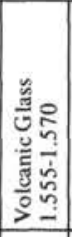 & 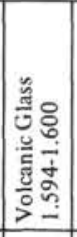 & 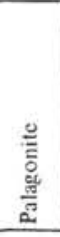 & 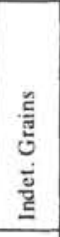 & 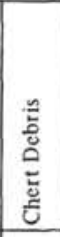 & 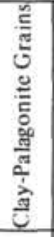 & 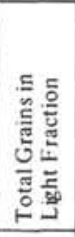 & 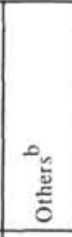 \\
\hline $53.1-2-2,145$ & 1 & & & & 24 & & & & & 4 & & & & & & & & & 83 & & 77 & 13 & 13 & & 2 & & & & 217 & \\
\hline $53.1-3-1$ & 1 & 1 & 1 & 30 & & & & & & & 1 & & & & & & 3 & & 215 & & 33 & 11 & 22 & & & & & & 318 & \\
\hline $53.1-3-2,40$ & 1 & & 1 & & 15 & & & & & & & & & & & & & & 168 & & 11 & 26 & 4 & & 1 & & & & 227 & \\
\hline $53.2-4-\mathrm{CC}$ & 4 & 2 & 2 & 31 & & & & & & 3 & 2 & 2 & & 3 & 24 & 14 & & 22 & & & 3 & 3 & & 5 & 26 & 55 & & & 246 & $\begin{array}{l}1^{\mathrm{M}} \\
44^{\mathrm{R}}\end{array}$ \\
\hline $53.2-5-\mathrm{CC}$ & 2 & & 1 & & 18 & & & & & 5 & 43 & & 95 & 42 & 69 & 36 & & 27 & & & 1 & & 2 & & 36 & 23 & & & 400 & \\
\hline $54.0-1-1,5$ & & & 2 & 25 & & & & & & 1 & 3 & & & & & & 21 & & & & 116 & 26 & 8 & & 1 & & & & 312 & $107 \mathrm{R}$ \\
\hline $54.0-1-2,7$ & & & & 12 & & & & & & 2 & 4 & & & & & & & 2 & 69 & & 171 & 31 & 15 & & & & & & 306 & \\
\hline $54.0-3-1,140$ & 1 & 2 & & 27 & & & & & & 1 & 5 & 1 & & 2 & 3 & & & 2 & & & 164 & 2 & 2 & & 4 & & & & 285 & $\begin{array}{l}1^{\mathrm{M}} \\
68^{\mathrm{R}}\end{array}$ \\
\hline $54.0-6-1,145$ & 1 & & 2 & 5 & & & & & & 1 & 3 & & & & & & & & & & 263 & 9 & 15 & & 2 & 1 & & & 324 & $22^{\mathrm{R}}$ \\
\hline $\begin{array}{l}55.0-3-4,145 \\
\text { after acid }\end{array}$ & 1 & 1 & & & 2 & & & 2 & & 336 & $\begin{array}{r}1 \\
75\end{array}$ & 35 & & & 4 & & $\begin{array}{l}2 \\
9\end{array}$ & 1 & & & $\begin{array}{l}1 \\
2\end{array}$ & 1 & 1 & & & 10 & & & $\begin{array}{l}346 \\
138\end{array}$ & \\
\hline $\begin{array}{l}55.0-9 \text {, Top } \\
\text { after acid }(5 \%)\end{array}$ & $\begin{array}{l}1 \\
1\end{array}$ & $\begin{array}{l}1 \\
1\end{array}$ & $\begin{array}{r}246 \\
2\end{array}$ & & & & & & & 99 & $\begin{array}{r}111 \\
99\end{array}$ & & & & 2 & 2 & $\begin{array}{r}2 \\
11\end{array}$ & 3 & 15 & & $\begin{array}{l}30 \\
77\end{array}$ & 1 & 3 & & & & & & $\begin{array}{l}492 \\
215\end{array}$ & \\
\hline $\begin{array}{l}55.0-13-3,40 \\
\text { after acid }\end{array}$ & & & & & $\begin{array}{r}1 \\
22\end{array}$ & & & & & 243 & $\begin{array}{l}14 \\
49\end{array}$ & 24 & & 15 & & & 6 & & $\begin{array}{r}12 \\
105\end{array}$ & & $\begin{array}{l}27 \\
71\end{array}$ & $\begin{array}{l}3 \\
7\end{array}$ & 21 & & & 3 & & & $\begin{array}{l}306 \\
317\end{array}$ & \\
\hline $55.2-2-3, \mathrm{CC}$ & 1 & & 1 & 48 & & 1 & & & & 5 & & 3 & & 7 & 12 & 14 & & 32 & & & 2 & 1 & 2 & & 68 & 41 & & & 306 & $68^{\mathrm{R}}$ \\
\hline 55.2-3, Тор & & & 1 & & 4 & & & & & & 18 & & & & 3 & 5 & 99 & 1 & & & 2 & & 1 & & & 25 & 115 & 55 & 329 & \\
\hline $57.1-3-1,17$ & & & 1 & 9 & & & & & & 145 & 1 & & & 1 & 1 & & & & & & 2 & 1 & & 83 & & & & & 318 & $74 \mathrm{R}$ \\
\hline $57.1-4-4,145$ & & & & 1 & & & & & 194 & 158 & 9 & & & & & & & & & & & & & 50 & & & & & 429 & $\begin{array}{l}{ }_{1}^{\mathrm{P}} \\
16^{\mathrm{R}}\end{array}$ \\
\hline after acid $(5 \%)$ & & & 1 & 3 & & & & & & & 4 & & & & & & 6 & & & 2 & & 1 & & 142 & & & & & 242 & $\begin{array}{c}1^{\mathrm{P}} \\
82^{\mathrm{R}}\end{array}$ \\
\hline $58.2-1-5,0$ & & & & & 28 & & & & 1 & & 2 & 3 & & & & & & & 98 & & 1 & & & 166 & 9 & & & & 308 & \\
\hline $58.2-1-6,95$ & 1 & & 11 & 7 & & & & & 4 & 6 & 4 & 6 & & 1 & 2 & & & 2 & & & 5 & 147 & & 8 & 2 & 2 & & & 270 & $61^{R}$ \\
\hline $59.1-3-C C$ & 1 & & 2 & 17 & & & & & & 2 & 1 & 2 & & 6 & 4 & 9 & & 36 & & & 3 & & & 32 & 65 & 50 & & & 268 & $37^{R}$ \\
\hline $59.2-1-\mathrm{CC}$ & 1 & 2 & & & & & & & & & 134 & & & & 2 & 2 & 25 & & 18 & & 42 & & 1 & & 11 & & & & 238 & \\
\hline
\end{tabular}


TABLE 2B - Continued

\begin{tabular}{|c|c|c|c|c|c|c|c|c|c|c|c|c|c|c|c|c|c|c|c|c|c|c|c|c|c|c|c|c|c|c|}
\hline $\begin{array}{c}\text { Sample } \\
\text { Designation }^{a}\end{array}$ & 苞 & 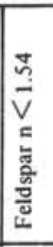 & 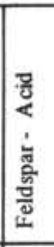 & 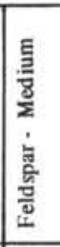 & 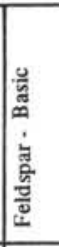 & 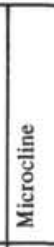 & 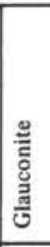 & 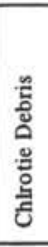 & 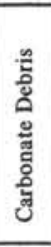 & 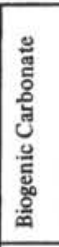 & 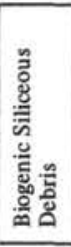 & 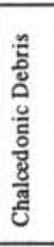 & 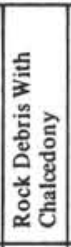 & 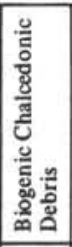 & 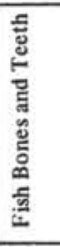 & 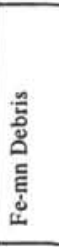 & 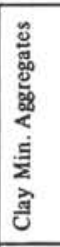 & 气ัँ & $\begin{array}{l}\text { 范 } \\
0 \\
\frac{0}{2} \\
\frac{8}{3} \\
>0 \\
\end{array}$ & 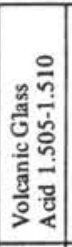 & 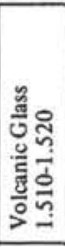 & 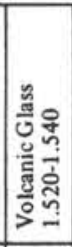 & 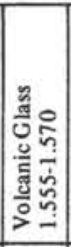 & 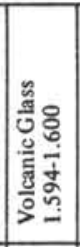 & 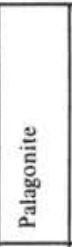 & 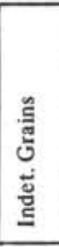 & 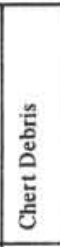 & 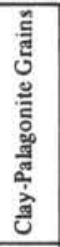 & 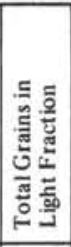 & 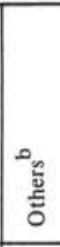 \\
\hline $59.2-2-C C$ & 1 & & & & 2 & & & & & & 252 & & & & 2 & 4 & 26 & & & & 7 & & 1 & & & & & & 294 & \\
\hline 59.2-6, Top & & & & & 1 & & & & & 29 & 3 & & & & 25 & 7 & & 248 & & & 9 & & & 19 & 6 & 13 & & & 360 & \\
\hline $60.0-1-\mathrm{CC}$ & 1 & & & 22 & & & & & & & 4 & & & & & & & 1 & 17 & & 31 & 4 & 1 & & 107 & & & & 188 & \\
\hline $60.0-2-\mathrm{CC}$ & & & & 35 & & & & & & 1 & 3 & & & & & & & & 70 & & 118 & 21 & 7 & & 2 & 4 & & & 261 & \\
\hline $60.0-3-\mathrm{CC}$ & 1 & & & 17 & & & & & & 14 & 2 & & & & & & & & 189 & & 30 & 16 & 11 & & 2 & & & & 282 & \\
\hline $60.0-4-C C$ & & 1 & & 24 & & & & & & 4 & & & & & & & & & 131 & & 99 & 9 & 17 & & 2 & & & & 287 & \\
\hline $60.0-4-\mathrm{CC}$ & & & & 15 & & & & & & & & & & & & & & & & & 241 & 47 & 14 & & 1 & & & & 403 & $84 \mathrm{R}$ \\
\hline $60.0-4-2,145$ & & & & 10 & & & & & & & & 2 & & & & & 31 & 1 & & & 233 & 24 & 9 & & 1 & & & & 366 & $54 \mathrm{R}$ \\
\hline $\begin{array}{l}60.0-5-\mathrm{CC} \\
\text { after acid }\end{array}$ & & & $\begin{array}{l}1 \\
1\end{array}$ & $\begin{array}{r}7 \\
18\end{array}$ & & & & & & $\begin{array}{l}2 \\
1\end{array}$ & $\begin{array}{l}1 \\
1\end{array}$ & & & & 1 & & $\begin{array}{r}90 \\
7\end{array}$ & 1 & & & $\begin{array}{l}144 \\
116\end{array}$ & $\begin{array}{r}1 \\
19\end{array}$ & $\begin{array}{r}9 \\
25\end{array}$ & & $\begin{array}{l}1 \\
2\end{array}$ & & & & $\begin{array}{l}299 \\
341\end{array}$ & $\begin{array}{l}40^{\mathrm{R}} \\
50^{\mathrm{R}}\end{array}$ \\
\hline $60.0-7-1,81.5$ & 2 & 1 & & 10 & 13 & & & & & 10 & 3 & & & & 1 & & 99 & & 51 & & 142 & & 20 & & 2 & & & & $\begin{array}{l}341 \\
331\end{array}$ & $8 \mathrm{~V}$ \\
\hline
\end{tabular}

ata on samples which were treated with $\mathrm{HCl}$ follows such samples and is indicated by "after acid."

bother minerals: $\mathrm{A}=$ Alloy $; \mathrm{K}=$ Kyanite $; \mathrm{L}=$ Limonite $\mathrm{M}=$ Muscovite $\mathrm{O}=$ Opal debris; $\mathrm{R}=$ Rock debris $\mathrm{P}=$ Palagonite $; \mathrm{V}=$ Volcanic glass basic 
TABLE 3A

Mineralogical Analysis of Heavy Fraction in Size Range $0.1-0.05 \mathrm{~mm}$

\begin{tabular}{|c|c|c|c|c|c|c|c|c|c|c|c|c|c|c|c|c|c|c|c|c|c|c|c|c|c|c|c|c|c|c|c|}
\hline $\begin{array}{c}\text { Sample } \\
\text { Designation }\end{array}$ & 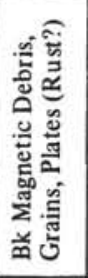 & 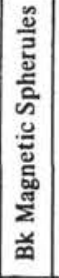 & 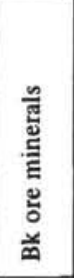 & 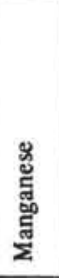 & 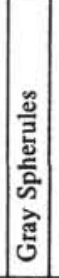 & $\overrightarrow{\underline{\underline{p}}}$ & 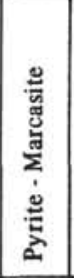 & 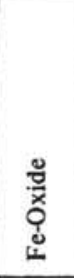 & 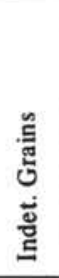 & $\begin{array}{l}\text { ๖్ } \\
\text { گ్ల }\end{array}$ & 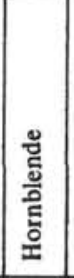 & 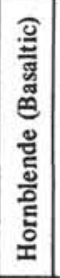 & 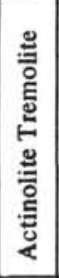 & 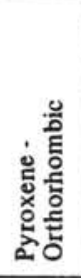 & 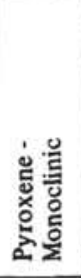 & 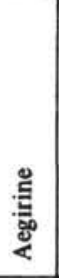 & 芯 & 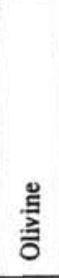 & 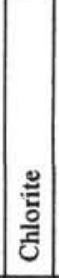 & 号 & 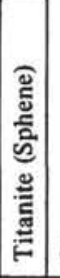 & 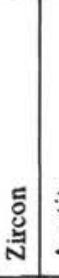 & : & 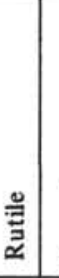 & 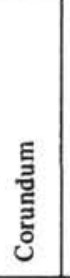 & 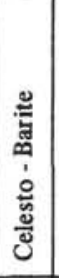 & 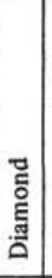 & 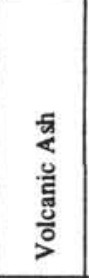 & 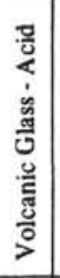 & . & 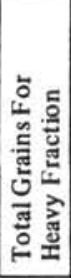 \\
\hline $45.0-1-4,145$ & & & 227 & - & & & - & 47 & & - & 1 & - & & - & 2 & & - & 1 & -1 & - & & & - & & & 1 & & - & -1 & & 279 \\
\hline $45.1-1-1,7$ & & & 98 & - & & & - & 16 & & - & - & - & & - & - & & - & - & - & - & & & - & & & - & & 126 & - & & 114 \\
\hline $45.1-1-2$ & 81 & & 4 & - & - & & & & - & - & - & -1 & - & 1 & 0 & - & 2 & - & - & -1 & - & - & - & & - & & - & & & - & 94 \\
\hline $46.0-1-1,145$ & 347 & & 3 & 7 & & & & 34 & & & 1 & & 1 & 9 & & & & & & & 1 & & & & & & & & & 1 & 412 \\
\hline $46.0-1-2,145$ & & & 51 & - & & & - & 3 & & - & - & - & & - & 1 & & - & - & - & - & & & - & & - & - & & - & & & 55 \\
\hline $46.0-1-3,145$ & 40 & 2 & 3 & 7 & - & & & - & & & - & & - & 2 & - & & - & & & - & - & & - & & - & - & - & & & 1 & 55 \\
\hline $46.0-1-5,145$ & 652 & - & - & - & 2 & & & - & & & - & & - & 3 & 1 & & - & & & - & - & & - & & - & - & - & & & - & 87 \\
\hline $46.0-1-6$ & & & 88 & 16 & & & 1 & 19 & & - & - & - & & - & 1 & & - & - & - & - & & & - & & - & - & & - & & & 125 \\
\hline $47.0-1-4,90$ & - & - & 23 & - & - & - & - & 9 & - & - & 1 & - & - & 20 & 16 & - & - & - & - & - & - & - & -- & - & - & - & 1 & - & - & - & 87 \\
\hline after $\mathrm{HCl}$ & - & - & - & - & - & - & - & - & - & - & - & - & - & - & - & - & - & - & - & 1 & - & - & $-1-$ & - & - & - & - & - & - & - & - \\
\hline $47.2-1-2,181$ & - & - & 5 & - & - & - & 27 & - & 2 & - & 1 & - & - & 2 & 17 & - & - & - & - & - & - & - & -- & - & - & - & - & 2 & - & - & 54 \\
\hline after $\mathrm{HCl}$ & - & - & - & - & -1 & - & - & - & - & - & - & - & - & - & - & - & - & - & - & - & - & - & $-1-$ & - & - & - & - & 84 & -1 & - & - \\
\hline $47.2-1-3,90$ & - & - & 12 & - & - & - & 5 & 3 & 4 & - & - & - & - & 2 & 6 & - & - & - & - & - & - & - & $-1-$ & - & - & - & - & - & -1 & - & 32 \\
\hline after $\mathrm{HCl}$ & - & - & - & - & - & - & - & - & - & - & - & - & - & - & - & - & - & - & - & - & - & - & $-1-$ & - & - & - & - & 17 & - & - & - \\
\hline $47 \cdot 2-2-2,8$ & - & - & 14 & - & - & - & - & 16 & 2 & - & - & - & - & 13 & 21 & - & - & - & - & - & - & - & $-1-$ & - & - & - & - & 14 & - & - & 74 \\
\hline after $\mathrm{HCl}$ & - & - & - & - & - & - & - & - & - & - & - & - & - & - & - & - & - & - & - & - & - & -1. & $-1-$ & - & - & - & - & 66 & - & - & - \\
\hline $47 \cdot 2-2-4,7$ & - & - & - & - & - & - & - & - & - & - & - & - & - & - & - & - & - & - & - & - & - & - & $-1-$ & - & - & - & -1 & 1 & - & - & - \\
\hline after $\mathrm{HCl}$ & - & - & - & - & - & - & - & - & - & - & - & - & - & - & - & - & - & - & - & - & - & - & - & - & - & - & - & 10 & - & - & - \\
\hline $47.2-2-6,6$ & - & - & - & - & - & - & - & - & - & - & - & - & - & - & - & - & - & - & -1 & - & - & - & $-1-$ & - & - & -1 & - & 7 & - & - & - \\
\hline after $\mathrm{HCl}$ & - & - & - & - & - & - & - & - & - & - & - & - & - & - & - & - & - & - & - & - & - & - & - & - & - & - & - & 41 & - & - & - \\
\hline $47.2-2-2,47$ & 6 & - & 27 & - & 1 & - & |- & - & 2 & - & 1 & - & - & ? & 51 & - & 1 & - & - & - & |- & - & $-1-$ & - & 1 & - & 1 & - & - & -1 & 116 \\
\hline $47 \cdot 2-3-3,6$ & - & - & 9 & - & - & - & - & 2 & - & - & - & -1 & - & 3 & 7 & - & 1 & - & - & - & - & - & -1. & - & - & - & - & 17 & - & - & 34 \\
\hline after $\mathrm{HCl}$ & - & - & - & - & - & - & - & - & - & - & - & - & - & - & - & - & - & - & - & - & - & - & - & - & - & - & - & 16 & - & - & - \\
\hline $47.2-4-1,16$ & - & - & 19 & - & -1 & - & - & 5 & - & - & 1 & - & - & 17 & 28 & - & 1 & - & - & - & - & - & - & - & - & - & - & 161 & - & - & 181 \\
\hline after $\mathrm{HCl}$ & - & - & 10.4 & - & - & - & - & 2.8 & - & - & 0.6 & - & - & 9.3 & 15.3 & - & 0.6 & - & - & - & - & - & -1. & - & - & - & - & 241.0 & - & - & - \\
\hline $47.2-4-3,16$ & - & - & - & - & - & - & - & - & - & - & - & - & - & - & - & - & - & - & - & - & - & - & - & - & - & - & - & 12 & - & - & - \\
\hline after $\mathrm{HCl}$ & - & - & - & - & - & - & - & - & - & - & - & - & - & - & - & - & - & - & - & - & - & - & - & - & - & - & - & 158 & - & - & - \\
\hline $47.2-4-5,16.5$ & - & - & - & - & - & - & - & - & - & - & - & - & - & - & - & - & - & - & - & - & - & - & - & - & - & - & - & - & - & - & - \\
\hline after $\mathrm{HCl}$ & - & - & - & - & - & - & - & - & - & - & - & - & - & - & - & - & - & - & - & - & - & - & - & - & - & - & - & - & - & - & - \\
\hline $47.2-5-1,17$ & - & - & - & - & - & - & - & - & - & - & - & - & - & - & - & - & - & - & - & - & - & - & - & - & - & - & - & - & - & - & - \\
\hline after $\mathrm{HCl}$ & - & - & - & - & - & - & - & - & - & - & - & - & - & - & - & - & - & - & - & - & - & - & - & - & - & - & - & - & - & - & - \\
\hline $47.2-5-3,6$ & - & - & - & - & - & - & - & - & - & - & - & - & - & - & - & - & - & - & - & - & - & - & - & - & - & - & - & - & - & - & - \\
\hline after $\mathrm{HCl}$ & - & - & - & - & - & - & - & - & 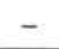 & - & - & - & - & - & - & - & - & - & - & - & - & - & -1. & - & - & - & - & - & - & - & - \\
\hline
\end{tabular}


TABLE 3A - Continued

\begin{tabular}{|c|c|c|c|c|c|c|c|c|c|c|c|c|c|c|c|c|c|c|c|c|c|c|c|c|c|c|c|c|c|c|c|}
\hline $\begin{array}{c}\text { Sample } \\
\text { Designation }\end{array}$ & 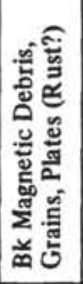 & 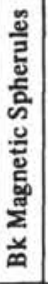 & 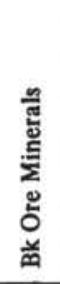 & 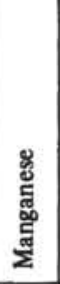 & 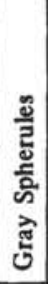 & $\stackrel{\underline{a}}{a}$ & 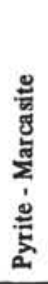 & 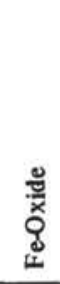 & 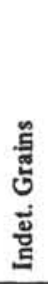 & ङ્ & 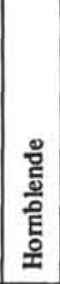 & 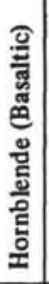 & 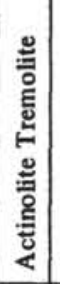 & 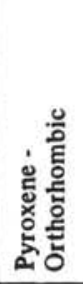 & 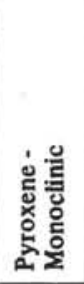 & 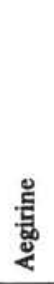 & 흠 & 竎 & 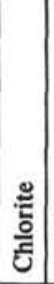 & : & 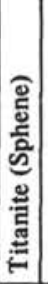 & 节 & 竎 & 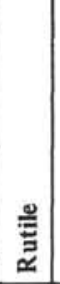 & 豆 & 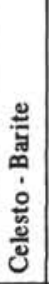 & 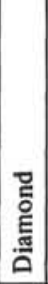 & 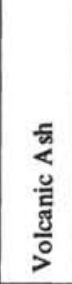 & 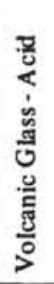 & 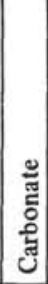 & 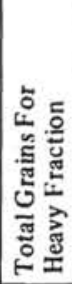 \\
\hline $47.2-5-5,6$ & - & - & - & 3 & - & - & - & 7 & - & - & - & - & - & 5 & 2 & - & - & - & - & - & - & - & - & - & - & - & - & - & - & - & 15 \\
\hline after $\mathrm{HCl}$ & - & - & - & - & - & - & - & - & - & - & - & - & - & - & - & - & - & - & - & - & -1 & - & - & - & - & - & - & - & - & - & - \\
\hline $47.2-6-2,6$ & 169 & - & 8 & 4 & - & - & - & 26 & - & - & 7 & - & - & 11 & 8 & - & 1 & - & - & 1 & - & - & 1 & - & 1 & 1 & - & - & - & - & 238 \\
\hline after $\mathrm{HCl}$ & - & - & - & - & - & - & - & - & - & - & - & - & - & - & - & - & - & - & - & - & - & - & - & - & - & - & - & - & - & - & - \\
\hline $47.2-7-1,17$ & - & - & 11 & 4 & - & - & - & $?$ & - & - & 2 & - & - & 34 & 4 & - & - & - & - & 1 & - & - & - & - & - & - & - & - & - & - & 621 \\
\hline after $\mathrm{HCl}$ & - & - & - & - & - & - & - & - & - & - & - & - & - & - & - & - & - & - & - & - & - & - & - & - & - & - & - & - & - & - & - \\
\hline $47.2-7-3,13$ & - & - & - & - & - & - & - & - & - & - & - & - & - & - & - & - & - & - & - & - & - & - & - & - & - & - & - & - & - & - & - \\
\hline $47.2-7-5,27.5$ & - & - & - & - & - & - & - & - & - & - & - & - & - & - & - & - & - & - & - & - & - & - & - & - & - & - & - & - & - & 1 & - \\
\hline $47.2-8-1,27$ & - & - & - & - & - & - & - & - & - & - & - & - & - & - & - & - & - & - & - & - & - & - & - & - & - & - & - & - & - & - & - \\
\hline $47.2-8-3,18$ & - & - & - & - & - & - & - & - & - & - & - & - & - & - & - & - & - & - & - & - & - & - & - & - & - & - & - & 2 & - & - & - \\
\hline $47.2-8-5,17$ & - & - & - & - & - & - & - & - & - & - & - & - & - & - & - & - & - & - & - & - & - & - & - & - & - & - & - & - & - & - & - \\
\hline $47.2-9-1,16$ & - & - & 70 & - & - & - & - & 45 & - & - & 1 & - & - & - & 1 & - & - & - & - & - & - & - & - & - & - & - & - & - & - & - & 117 \\
\hline $47.2-9-3,17$ & - & - & - & - & - & - & - & - & - & - & - & -1 & - & - & - & - & - & - & - & - & - & - & - & - & - & - & - & - & - & - & - \\
\hline $47.2-10-2,17$ & - & - & - & - & - & - & - & - & - & - & - & - & - & - & - & - & - & - & - & - & - & - & - & - & - & - & - & - & - & - & - \\
\hline $47.2-10-4,17$ & - & - & - & - & - & - & - & - & - & - & - & - & - & - & - & - & - & - & - & - & - & - & - & - & - & - & - & - & - & - & - \\
\hline $47.2-10-6,16$ & - & - & - & - & - & - & - & - & - & - & - & -1 & - & - & - & - & - & - & - & - & -1 & - & - & - & - & - & - & - & - & - & - \\
\hline $47.2-11-2,145$ & - & - & - & - & - & - & - & - & - & - & - & - & - & - & - & - & - & - & -1 & -1 & - & - & - & - & - & - & - & - & - & - & - \\
\hline after $\mathrm{HCl}$ & - & - & - & - & - & - & - & - & - & - & - & - & - & - & - & - & - & - & - & - & - & - & - & - & - & - & - & - & - & 2 & - \\
\hline $47.2-12-2,17$ & - & - & - & - & - & - & - & - & - & - & - & - & - & - & - & - & - & - & - & - & - & - & - & - & - & - & 1 & - & - & - & - \\
\hline after $\mathrm{HCl}$ & - & - & - & - & - & - & - & - & - & - & - & - & - & - & - & - & - & - & - & - & - & - & -1 & - & - & - & - & - & - & - & - \\
\hline $47.2-12-4,145$ & - & - & - & - & - & - & - & - & - & - & - & - & - & - & - & - & - & - & - & - & - & - & - & - & - & - & - & 3 & - & - & - \\
\hline $47.2-13-2,145$ & - & - & - & - & - & - & - & - & - & - & - & - & - & - & - & - & - & - & - & - & - & - & - & - & - & - & - & - & - & - & - \\
\hline $47.2-13-6,145$ & - & - & - & - & - & - & - & - & - & - & - & -1 & - & - & - & - & - & - & - & - & - & - & -1 & - & - & - & - & - & - & - & - \\
\hline $47.2-14-3,145$ & - & - & - & - & - & - & - & - & - & - & - & -1 & - & - & - & - & - & - & - & - & - & - & - & - & - & - & - & - & - & - & - \\
\hline $47.2-14-4,145$ & - & - & 7 & - & - & - & 1 & 7 & - & - & - & - & - & - & - & - & - & - & - & - & - & - & - & - & - & - & - & - & - & - & 15 \\
\hline after $\mathrm{HCl}$ & - & - & - & - & - & - & - & - & - & - & - & - & - & - & - & - & - & - & - & - & - & - & - & - & - & - & - & - & - & - & - \\
\hline $50.1-1,75 \mathrm{~cm}$ & 32 & 1 & 41 & & & & & & - & 1 & 12 & - & - & 3 & 4 & & 1 & - & - & 2 & - & & - & & - & 1 & & & & - & 138 \\
\hline $51.0-1-2$ & 8 & & 12 & 25 & - & & & & 3 & 1 & 9 & - & - & 4 & 11 & - & 3 & - & - & 2 & 1 & - & 1 & & - & & - & & & - & 79 \\
\hline $51.0-1-4,6$ & & & - & - & & & - & - & & - & - & - & & - & - & & - & - & - & - & & & - & & & - & & 136 & - & & - \\
\hline $51.0-1-6,16$ & & & 4 & 2 & & & - & 27 & & - & 2 & - & & - & 3 & & - & - & - & - & & & - & & - & - & & 7 & & & 38 \\
\hline $51.1-1-4$ & 9 & - & 89 & - & & & & & - & - & 11 & - & 6 & 40 & 98 & & 3 & - & 2 & 2 & 1 & & - & & - & - & & & & - & 316 \\
\hline $51.1-2-2$ & 90 & - & 15 & - & & & & & - & - & 4 & - & - & 1 & 8 & & - & - & - & 2 & - & & - & & - & - & & & & - & 119 \\
\hline
\end{tabular}


TABLE 3A - Continued

\begin{tabular}{|c|c|c|c|c|c|c|c|c|c|c|c|c|c|c|c|c|c|c|c|c|c|c|c|c|c|c|c|c|c|c|c|}
\hline $\begin{array}{c}\text { Sample } \\
\text { Designation }\end{array}$ & 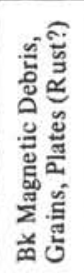 & 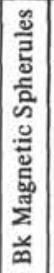 & 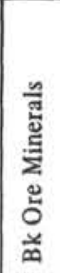 & 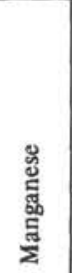 & 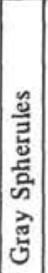 & $\overrightarrow{\underline{z}}$ & 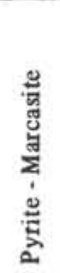 & 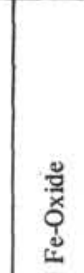 & 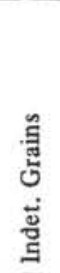 & 䓌 & 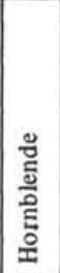 & 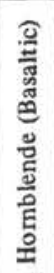 & 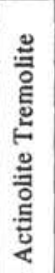 & 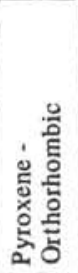 & 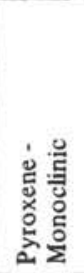 & 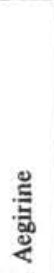 & $\begin{array}{l}\text { 总 } \\
\text { : }\end{array}$ & 竞 & 冚 & 苛 & 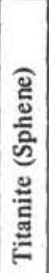 & 总 & 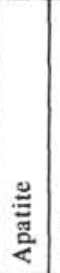 & 足 & 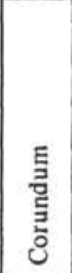 & 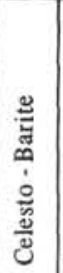 & 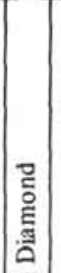 & 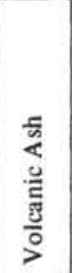 & 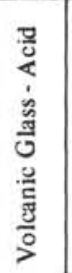 & 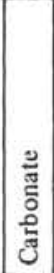 & 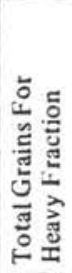 \\
\hline $52.0-1-2$ & 27 & - & 27 & - & & & & & - & - & 2 & - & - & 14 & 30 & & - & - & - & - & - & & - & & - & - & & & & - & 105 \\
\hline $52.0-2-5$ & 47 & - & 76 & - & & & & & 13 & 1 & 5 & - & 1 & 28 & 160 & & 6 & 2 & - & - & 1 & & 2 & & - & - & & & & 1 & 379 \\
\hline $52.0-3-5$ & 12 & - & 66 & - & & & & & 31 & - & 2 & - & - & - & 103 & & 2 & - & - & - & - & & 1 & & - & - & & & & - & 217 \\
\hline $52.0-4-2$ & 26 & 1 & 35 & 11 & 1 & & & & 16 & - & 12 & - & 1 & 2 & 5 & - & 16 & - & - & - & - & - & 1 & & - & & - & & & - & 146 \\
\hline $52.0-5-2$ & 68 & - & 64 & - & & & & & 36 & 3 & 2 & - & 3 & - & 4 & & 16 & 2 & 1 & 1 & -1 & & 3 & & - & - & & & & 1 & 202 \\
\hline $52.0-5-3$ & 22 & & 46 & 7 & 6 & & & & 28 & - & 2 & - & 1 & 1 & 9 & - & 13 & 3 & 2 & - & - & - & 3 & & - & & - & & & - & 142 \\
\hline $52.0-8-2$ & 22 & - & 9 & 57 & & & & & - & - & - & - & 1 & - & 1 & & - & - & & - & - & & - & & - & 1 & & & & - & 419 \\
\hline $52.0-8-4$ & 260 & 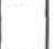 & 1 & - & - & & & & - & - & - & - & - & - & 3 & - & - & - & - & - & - & - & - & & - & & - & & & - & 204 \\
\hline $52.0-8-6$ & 28 & 2 & - & 103 & & & & & - & - & - & - & - & - & 2 & & - & - & & - & - & & - & & - & - & & & & - & 332 \\
\hline $53.0-0-2$ & - & - & - & - & - & & & - & & & - & & - & - & - & & - & & & 1 & - & & & & - & - & - & & & - & - \\
\hline $53.0-1-1$ & 18 & - & 69 & - & & & & & 90 & - & 3 & 1 & 1 & 48 & 193 & & 2 & 1 & & - & - & & 3 & & 1 & 1 & & & & - & 426 \\
\hline $53.0-7-1$ & 3 & & 13 & - & - & & & & 42 & - & 4 & - & - & 1 & 12 & - & - & 1 & & 3 & 1 & - & 1 & & - & & & & & 5 & 86 \\
\hline $53.1-1-1$ & 4 & & 9 & - & - & & & & - & - & & - & - & 3 & 12 & - & 1 & - & - & - & - & - & 1 & & 1 & & - & & & 1 & 39 \\
\hline $53.1-1-2$ & 6 & & 16 & 3 & - & & & & - & - & 2 & - & - & 4 & 39 & - & 2 & - & - & - & - & - & - & & - & & - & & & - & 74 \\
\hline $53.1-1-4,11$ & & & 138 & & & & - & 12 & & 1 & 2 & - & & 7 & 174 & & 2 & - & - & 1 & & & 1 & & 17 & -1 & & 51 & & & 355 \\
\hline $53.1-1-6,28$ & & & - & - & & & - & - & & - & - & - & & - & - & & - & - & 2 & & & & - & & - & -1 & & 42 & & & - \\
\hline $53.1-2-4$ & 4 & & 32 & - & - & & & & - & - & 6 & 2 & 1 & 52 & 167 & - & 2 & - & - & - & - & - & 1 & & - & & - & & & - & 416 \\
\hline $53.1-2-3,16$ & & & 122 & - & & & - & 22 & & - & 22 & - & & 20 & 38 & & 4 & 1 & - & - & & & 3 & & 49 & -1 & & 96 & & & 411 \\
\hline $53.1-2-6$ & 14 & & 55 & - & - & & & & 78 & - & 13 & - & 3 & 72 & 194 & - & 2 & - & 1 & & 1 & - & 1 & & - & & - & & & - & 434 \\
\hline $53.1-3-3,23$ & & & 55 & - & & & - & 24 & & - & - & 1 & & 62 & 125 & & 1 & - & - & 2 & & & - & & 124 & - & & 142 & & & 394 \\
\hline $53.1-3-5$ & 4 & & 45 & - & - & & & & - & - & 2 & - & - & 19 & 177 & - & 2 & - & - & - & - & - & 1 & & - & & - & & & - & 464 \\
\hline $53.2-1-1$ & 5 & & 3 & - & - & & & & - & - & 1 & - & - & 4 & 23 & - & - & - & - & 1 & - & - & - & & - & & - & & & 1 & 119 \\
\hline $53.2-1-3,23$ & & & 97 & - & & & - & 12 & & - & - & - & 97 & & 127 & & 3 & - & - & - & & & - & & 64 & - & & 161 & & & 400 \\
\hline $53.2-1-5,7$ & & & 111 & - & & & - & 27 & & - & 3 & - & & 65 & 154 & & 1 & - & - & - & & & 1 & & 57 & - & & 195 & & & 419 \\
\hline $53.0-6-2,66$ & & & 161 & - & & & - & 119 & & 1 & - & - & & 2 & 1 & & - & - & 1 & - & & & - & & & - & & 225 & - & & 294 \\
\hline $54.0-1-1$ & 9 & & 7 & - & - & & & & - & - & 1 & - & - & - & 7 & - & - & - & 1 & - & - & & - & & - & & & & & 1 & 24 \\
\hline $54.0-1-3,35$ & & & & - & & & - & 4 & & - & - & - & & 2 & 2 & & 1 & - & - & - & & & - & & & - & & 41 & & & \\
\hline $54.0-2-2$ & - & & 20 & 2 & - & & & & - & - & 2 & - & - & 16 & 124 & - & 2 & - & - & - & - & - & - & & - & & - & & - & 1 & 382 \\
\hline $54.0-4-1$ & 3 & & 39 & - & - & & & & - & - & 2 & - & - & 45 & 110 & - & 2 & - & - & - & - & - & - & & - & & - & & & - & 256 \\
\hline $54.0-7-2$ & 3 & & 6 & 63 & - & & & & - & - & - & - & 1 & 15 & 39 & - & 1 & - & - & - & - & - & - & & - & & - & & & - & 256 \\
\hline $54.0-6-2,16$ & - & & 20 & - & & & - & 5 & & - & 1 & - & & 35 & 162 & & 3 & - & - & - & & & 1 & & & -1 & & 28 & 11 & & 238 \\
\hline $54.0-6-4,16$ & & & 63 & - & & & - & 10 & - & - & 9 & - & & 45 & 210 & & 2 & - & - & - & & & 1 & & & - & & 106 & & & 446 \\
\hline $54.0-6-5,17$ & & & 5 & - & & & - & 2 & - & - & - & - & & 4 & 10 & & - & & - & - & & & - & & & - & & 15 & & & 36 \\
\hline
\end{tabular}


TABLE 3A - Continued

\begin{tabular}{|c|c|c|c|c|c|c|c|c|c|c|c|c|c|c|c|c|c|c|c|c|c|c|c|c|c|c|c|c|c|c|c|}
\hline $\begin{array}{c}\text { Sample } \\
\text { Designation }\end{array}$ & 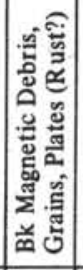 & 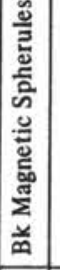 & 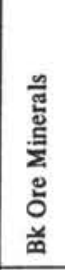 & 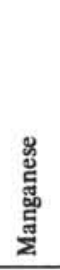 & 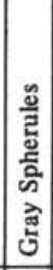 & $\begin{array}{l}\vec{w} \\
\vec{z} \\
\end{array}$ & 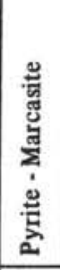 & 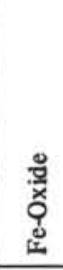 & 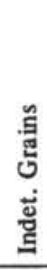 & 志 & $\begin{array}{l}\text { 를 } \\
\text { 율 } \\
\text { 통 } \\
\text { 오 }\end{array}$ & 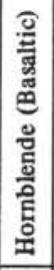 & 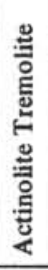 & 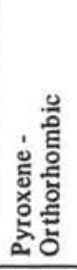 & 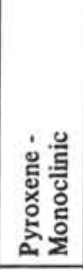 & 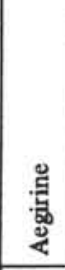 & $\begin{array}{l}\text { 음 } \\
\text { 몸 }\end{array}$ & 涪 & 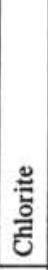 & 言 & 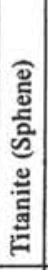 & 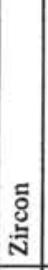 & 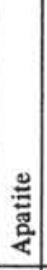 & 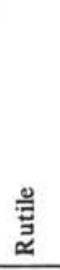 & 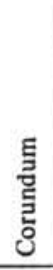 & 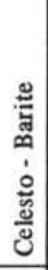 & 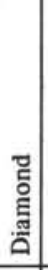 & 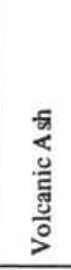 & 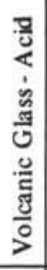 & 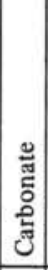 & 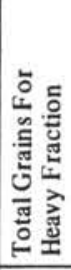 \\
\hline $55.0-1-2$ & & & - & - & & & - & - & - & - & - & - & & - & - & & - & - & - & - & & & - & & & - & & - & & & - \\
\hline $55.0-2-5,7$ & & & 7 & & & & - & - & - & - & - & & & 2 & 7 & & - & & - & & & & & & & - & & 36 & & & 17 \\
\hline $55.0-2-6$ & - & - & - & - & - & & & - & & & - & & - & - & - & & - & & & - & - & & - & & - & - & - & & & - & - \\
\hline $55.0-4-2,145$ & & & - & & & & - & - & - & - & - & & & - & - & & - & & - & & & & & & & - & & 25 & & & - \\
\hline $55.0-8-1$ & - & - & - & - & - & & & - & & & - & & - & - & - & & - & & & - & - & & - & & - & - & - & & & - & - \\
\hline $55.0-8-5,18$ & & & 7 & & & & - & & 2 & - & - & & & 1 & 2 & & 1 & & - & & & & & & & - & & 30 & & & 19 \\
\hline $55.0-10-3$ & - & - & - & - & - & & & - & & & - & & - & - & - & & - & & & - & - & & - & & - & - & - & & & - & - \\
\hline $55.0-11-1,46$ & & & 6 & & & & - & 5 & - & - & 1 & & & 2 & 3 & & - & & - & & & & & & & - & & 107 & & & 62 \\
\hline $55.0-11-5$ & - & - & - & - & - & & & - & & & - & & - & - & - & & - & & & - & - & & - & & - & - & - & & & - & - \\
\hline $55.0-12-2$ & - & - & - & - & - & & & - & & - & - & & - & - & - & & - & & & - & - & & - & & - & - & - & & & - & - \\
\hline $55.0-12-5,7$ & & & 45 & & & & - & - & - & - & - & & & 3 & 13 & & - & & - & & & & & & & - & & 376 & & & 267 \\
\hline $55.0-13-3,7$ & & & 1 & & & & - & 12 & - & - & - & & & - & 13 & & - & & - & & & & & & & - & & 125 & & & 98 \\
\hline $55.0-14-1$ & - & - & 5 & 1 & - & & & 12 & & & 2 & & - & 90 & 2 & & - & & & - & - & & - & & - & - & - & & & - & 343 \\
\hline $55.0-14-3,145$ & & & 15 & & & & - & 11 & - & 1 & 2 & & & - & 4 & & - & & - & & & & & & & - & & 172 & & & 162 \\
\hline $55.0-14-6$ & - & - & 3 & - & - & & & 6 & & & 1 & & & 5 & - & & - & & & - & - & & - & & - & - & - & & & - & \\
\hline $56.2-1-2$ & 7 & - & 4 & & & & & & & 2 & 2 & - & - & 1 & 90 & & 2 & - & - & - & 1 & & 1 & & - & & & & & - & 473 \\
\hline $56.2-1-4$ & - & & - & - & - & & & & - & - & 1 & - & - & 2 & 5 & - & - & - & - & - & - & - & - & & - & & - & & & 2 & 9 \\
\hline $56.2-2-2,90$ & & - & 80 & - & - & & 13 & 18 & - & - & - & - & & - & - & - & - & - & - & 1 & & - & - & & - & & & - & - & & 112 \\
\hline $56.2-2-6,16$ & & & - & - & & & - & - & - & - & - & - & & - & - & & - & - & - & - & & & - & & & - & & - & & & - \\
\hline $56.2-3-2,7$ & & - & 37 & - & - & & - & 4 & - & 5 & 13 & - & & 3 & 44 & 2 & 9 & - & - & 3 & & 2 & 3 & & - & & & 277 & - & & 402 \\
\hline $56.2-3-2,145$ & & - & - & - & - & & - & - & - & - & - & - & & - & - & - & - & - & - & - & & - & - & & - & & & - & - & & - \\
\hline $56.2-4-2,10$ & & & 19 & & & & - & & & & 1 & - & & - & 1 & & - & - & - & - & & & - & & & - & & - & & & 22 \\
\hline $56.2-4-5,13$ & & - & 12 & - & - & & - & 6 & 6 & 13 & 17 & - & & 2 & 13 & 1 & 4 & - & - & - & & - & 3 & & - & & & - & - & & 77 \\
\hline $56.2-6-4$ & 7 & & 17 & - & - & & & & 2 & 3 & 3 & - & 1 & 1 & 39 & 1 & 2 & 1 & - & 1 & - & 1 & 1 & & 2 & & - & & & - & 353 \\
\hline $56.2-6-6,16$ & & - & 7 & - & - & & - & - & - & 9 & 25 & - & & 6 & 11 & 1 & 7 & - & - & 5 & & - & - & & - & & & 214 & - & & 285 \\
\hline $56.2-7-2,43$ & & - & 31 & - & - & & - & 38 & 7 & 2 & 4 & - & & 2 & 6 & - & 1 & - & - & 1 & & 1 & 2 & & - & & & 349 & - & & 444 \\
\hline $56.2-7-4,39$ & & - & 102 & - & - & & - & 24 & - & 1 & 11 & 7 & & 2 & 147 & 16 & 10 & - & - & - & & - & - & & - & & & 90 & - & & 410 \\
\hline $56.2-8-2,7$ & & & 49 & - & & & - & 31 & - & 4 & 3 & - & & 2 & 37 & & 2 & - & - & - & & & 1 & & & - & & 161 & & & 290 \\
\hline $56.2-9-6,8$ & & - & 32 & - & - & & - & 7 & - & - & 2 & - & & 5 & 39 & 5 & 2 & - & - & 1 & & 3 & - & & - & & & 150 & - & & 246 \\
\hline $56.2-10-4$ & 3 & - & 10 & & & & & & & - & 1 & - & 1 & 1 & 48 & & 1 & - & 1 & 1 & - & & 1 & & - & & & & & - & 393 \\
\hline $56.2-5-4$ & - & - & - & & & & & & & - & - & - & - & - & - & & - & - & - & - & - & & - & & - & & & & & - & \\
\hline $57.1-1-2,0$ & & - & 41 & - & - & & - & 18 & - & - & 3 & - & & 2 & 143 & - & 2 & - & - & - & & - & - & & - & & & 236 & - & & 445 \\
\hline
\end{tabular}


TABLE 3A - Continued

\begin{tabular}{|c|c|c|c|c|c|c|c|c|c|c|c|c|c|c|c|c|c|c|c|c|c|c|c|c|c|c|c|c|c|c|}
\hline $\begin{array}{c}\text { Sample } \\
\text { Designation }\end{array}$ & 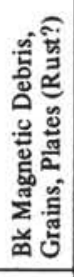 & 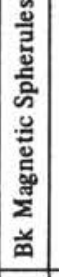 & 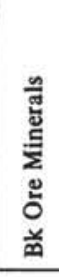 & $\begin{array}{l}8 \\
\frac{8}{4} \\
\frac{9}{4} \\
\frac{5}{2}\end{array}$ & 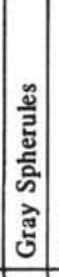 & $\begin{array}{l}\overrightarrow{\underline{m}} \\
\overrightarrow{\tilde{a}}\end{array}$ & 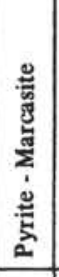 & 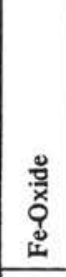 & 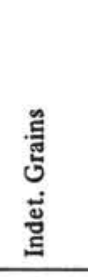 & 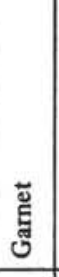 & 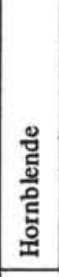 & 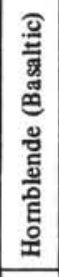 & 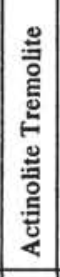 & 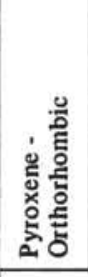 & 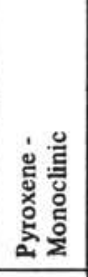 & 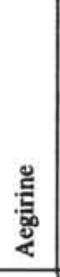 & 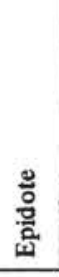 & 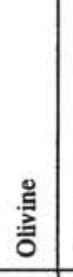 & 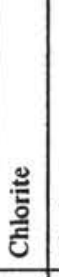 & 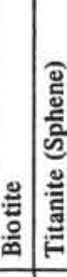 & $\frac{8}{\mathrm{~N}}$ & 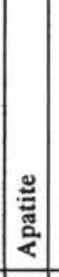 & $\begin{array}{l}0 \\
\bar{z} \\
\Sigma\end{array}$ & 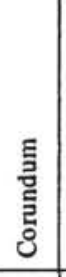 & 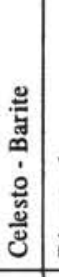 & 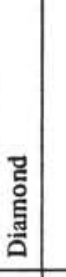 & 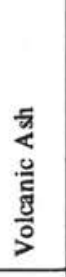 & 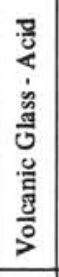 & 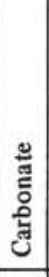 & 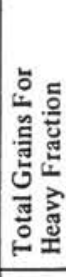 \\
\hline $57.1-1-3$ & 1 & 1 & 1 & & & & & & & 1 & 2 & - & - & - & 12 & & 1 & - & - & $-1-$ & & - & & - & & & & & - & 336 \\
\hline $57.1-1-5,7$ & & - & - & - & - & & - & - & - & - & - & - & & - & - & - & - & - & -1 & - & - & - & & - & & & - & - & & - \\
\hline $57.1-1-8,7$ & & & - & - & & & - & - & - & - & - & - & - & & - & & - & - & - & - & & - & & & - & & - & & & - \\
\hline $57.1-2-1,44$ & & & 46 & - & & & - & 12 & - & - & - & - & 2 & & 15 & & 1 & - & - & - & & - & & & - & & 278 & & & 354 \\
\hline $57.1-3-2,108$ & & & 27 & - & & & - & 9 & 6 & 1 & 1 & - & 2 & & 15 & & 2 & - & -1 & - & & - & & & - & & 347 & & & 380 \\
\hline $57.2-1-1$ & - & 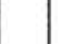 & - & - & -1 & - & & & - & - & - & - & - & - & - & - & - & - & - & $-1-$ & & - & & & & - & & & - & - \\
\hline 58. $-1-3,145$ & & - & 6 & - & 1 & & - & 7 & 15 & - & 3 & - & & - & 93 & - & 1 & 148 & -1 & - & - & - & & - & & & - & - & & 274 \\
\hline $58.2-1-3,16$ & & - & 24 & - & - & & - & 13 & - & 1 & 3 & - & & 2 & 30 & 1 & 2 & - & - & 1 & - & 2 & & - & & & 281 & - & & 360 \\
\hline $58.2-1-5$ & - & - & - & & & & & & & - & 1 & 1 & - & 2 & 16 & & - & - & - & $-1-$ & & -1 & & 1 & & & & & - & 129 \\
\hline $59.1-3-2,11$ & & - & 66 & - & - & & - & 113 & 9 & - & 4 & - & & 29 & 9.9 & - & 2 & 2 & - & - & - & 1 & & - & & & 58 & - & & 383 \\
\hline $59.1-3-5,146$ & & - & - & - & - & & - & - & - & - & - & - & & - & - & - & - & - & - & - & - & - & & - & & & - & - & & - \\
\hline $59.2-1-2,4$ & & 1 & 41 & - & - & & - & 58 & 118 & - & 2 & - & & 10 & 115 & - & 4 & - & - & - & 1 & -1 & & 1 & & & 115 & - & & 466 \\
\hline $59.2-2-3$ & 5 & - & 2 & & & & & & & - & 1 & - & - & 2 & 49 & & - & - & - & $-1-$ & & 1 & & - & & & & & - & 319 \\
\hline $59.2-2-5,35$ & & - & 9 & - & - & & - & 6 & 6 & - & 1 & - & & - & 5 & - & - & - & -1 & - & - & - & & - & & & - & - & & 27 \\
\hline $59.2-3-2$ & 11 & - & 25 & & & & & & & - & 1 & - & - & 5 & 134 & & - & - & 1 & $1-$ & & 1 & & - & & & & & - & 250 \\
\hline $60.0-1-1,7$ & & - & 48 & & - & & - & 14 & 17 & - & 15 & - & & 32 & 274 & - & 3 & 1 & 1 & - & - & - & & - & & & 42 & - & & 405 \\
\hline $60.0-2-1$ & 2 & - & 121 & & & & & & 6 & - & 2 & -1 & - & 104 & 116 & & - & - & 1 & $-1-$ & & 2 & & - & & & & & - & 357 \\
\hline $60.0-3-2,8$ & & - & 19 & & - & & - & 10 & 5.0 & 1 & 3 & - & & 32 & 47 & - & - & - & -1 & 1 & - & - & & - & & & 86 & 2 & & 121 \\
\hline $60.0-5-1,51$ & & - & 6 & & - & & - & - & - & - & 8 & - & & 4 & 5 & - & - & - & - & - & - & - & & - & & & 210 & - & & 30 \\
\hline $60.0-5-3,40$ & & - & 31 & & - & & - & 42 & - & - & 2 & - & & 13 & 81 & - & 4 & 1 & 2 & - & - & 1 & & - & & & 244 & 51 & & 417 \\
\hline $60.0-6-2$ & 2 & - & 2 & & & & & & & - & 1 & - & - & 16 & 37 & & 2 & - & - & $-1-$ & & 1 & & & & & & & 1 & 98 \\
\hline $60.0-6-4,25$ & & - & - & & - & & - & - & - & - & - & - & & - & - & - & - & - & - & - & - & - & & - & & & 131 & - & & - \\
\hline $60.0-6-6$ & 5 & - & 20 & & & & & & & 1 & 2 & - & 1 & 55 & 244 & & 6 & 1 & 2 & -1 & & 1 & & - & & & & & - & 414 \\
\hline $60.0-8-1$ & 2 & - & 13 & & & & & & & 1 & 1 & - & - & 19 & 196 & & - & 1 & - & $-1-$ & & 1 & & - & & & & & - & 291 \\
\hline $60.0-8-3,145$ & & - & 65 & & - & & - & 3 & - & - & 5 & - & & 37 & 242 & - & 4 & 1 & - & - & - & 3 & & - & & & 205 & - & & 454 \\
\hline $60.0-9-5$ & 3 & & 130 & - & - & & & & - & - & 6 & - & 2 & 73 & 205 & 1 & - & - & 1 & $-1-$ & $-1-$ & 2 & & - & & - & & & - & 435 \\
\hline $60.03-\mathrm{CC}$ & & & - & & & & - & - & & - & - & & & - & - & & - & & -1 & & & & & & & & 243 & & & - \\
\hline
\end{tabular}


TABLE 3B

Mineralogical Analysis of Light Fraction in Size Range 0.1-0.05 mm

\begin{tabular}{|c|c|c|c|c|c|c|c|c|c|c|c|c|c|c|c|c|c|c|c|c|c|c|c|c|c|c|c|c|c|c|c|c|}
\hline $\begin{array}{c}\text { Sample } \\
\text { Designation }\end{array}$ & 莺 & 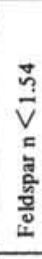 & 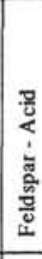 & 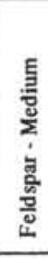 & 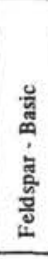 & $\begin{array}{l}\text { 总 } \\
\frac{0}{0} \\
\frac{0}{\Sigma}\end{array}$ & $\begin{array}{l}\text { 气̆ } \\
8 \\
\frac{\text { gू }}{0} \\
\end{array}$ & 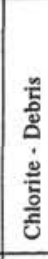 & 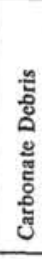 & 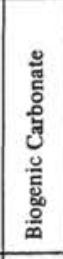 & 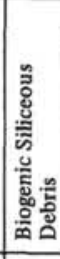 & 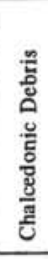 & 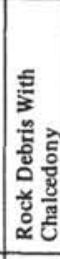 & 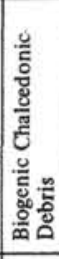 & 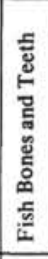 & 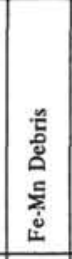 & 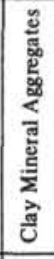 & 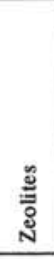 & $\begin{array}{l}\frac{5}{2} \\
\frac{0}{\tilde{j}} \\
\frac{0}{0} \\
>\end{array}$ & 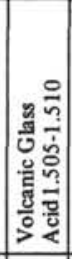 & 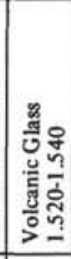 & 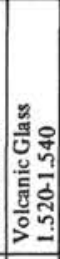 & 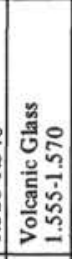 & 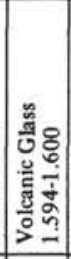 & 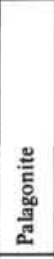 & 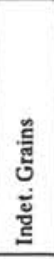 & 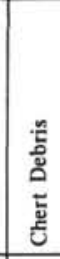 & 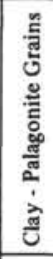 & 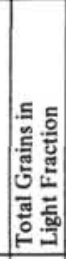 & \multicolumn{3}{|c|}{ ప } \\
\hline $45.0-1-4,145$ & 1 & - & - & & - & - & & 1 & & - & - & 57 & 39 & 119 & 6 & - & 11 & - & & & - & - & - & - & - & - & - & - & & 234 & & \\
\hline $45.1-1-1,7$ & 2 & 4 & - & & 11 & - & & 1 & & 4 & 1 & - & - & - & 52 & 2 & 32 & - & & & 8 & - & 1 & 11 & - & 4 & - & - & & 261 & 20 & \\
\hline $45.1-1-2$ & 4 & 1 & 4 & 18 & 18 & - & - & 7 & - & - & - & - & - & - & 19 & 39 & & 2 & & & 2 & - & & & & - & 19 & & - & 142 & $\underset{17}{9 \mathrm{P}}$ & ${ }_{1} \mathrm{P}$ \\
\hline $46.0-1-1,145$ & 1 & & 1 & 1 & 1 & & & 1 & & 119 & 270 & 8 & 17 & & & & & & & & 1 & & & & & & & & & 423 & $7 \mathrm{R}$ & $2+2 \mathrm{~V}$ \\
\hline $46.0-1-2,145$ & 2 & 2 & - & - & 2 & - & & & & - & 2 & 21 & 43 & 148 & 24 & 0.8 & 9 & 1 & & - & 3 & 1 & 3 & - & - & - & - & & & & 20 & \\
\hline $46.0-1-3,145$ & - & - & 1 & 2 & 2 & - & - & - & - & - & - & & 79 & 239 & 20 & - & 22 & 1 & & - & 1 & & - & - & & 1 & - & & 3 & 313 & $\begin{array}{l}-\mathrm{M} \\
{ }_{1} \mathrm{R} \\
{ }^{\mathrm{P}}\end{array}$ & ${ }_{-0}^{1+2 V}$ \\
\hline $46.0-1-5,145$ & - & - & - & 2 & 2 & - & & & 9 & 200 & 61 & - & - & - & 2 & - & & 1 & & - & 78 & & - & - & & - & - & & 8 & 365 & ${ }_{4} R$ & \\
\hline $46.0-1-6$ & - & - & - & & - & - & & - & & - & 2 & 91 & 43 & 90 & 10 & 6 & 24 & - & & & - & - & - & - & - & 2 & - & - & & 269 & 10 & \\
\hline $47.0-1-4,90$ & - & - & - & 2 & 2 & - & - & - & 9 & 200 & 61 & - & - & - & 2 & - & - & 1 & & - & 78 & - & - & - & - & - & - & - & 8 & 365 & & $17+4^{R}$ \\
\hline after $\mathrm{HCl}$ & - & - & 2 & 9 & 9 & - & - & - & - & - & 62 & - & - & - & - & - & - & - & & - & 234 & - & 5 & - & - & - & - & - & 8 & 407 & $\begin{array}{l}{ }_{1}^{\mathrm{V}} \\
1^{\mathrm{P}}\end{array}$ & $30^{\mathrm{R}}$ \\
\hline $47.2-1-2,181$ & - & - & - & - & 1 & - & - & - & - & 267 & 3 & - & - & - & 4 & - & 11 & - & & - & 3 & 1 & - & - & - & - & - & - & - & 292 & & \\
\hline after $\mathrm{HCl}$ & - & - & - & - & 29 & - & - & - & - & $?$ & 13 & - & - & - & - & - & 48 & 3 & & 1 & 51 & 31 & - & 1 & - & - & 1 & - & - & 270 & & \\
\hline $47.2-1-3,90$ & - & - & - & - & 3 & - & - & - & - & 247 & 1 & - & - & - & 2 & - & - & - & & - & 3 & - & - & - & - & - & - & - & - & 256 & & \\
\hline after $\mathrm{HCl}$ & - & 1 & - & - & 10 & - & - & - & - & 5 & 19 & - & - & - & - & - & 78 & 6 & & - & 86 & 5 & - & 6 & - & - & - & - & - & 233 & & \\
\hline $47.2 \cdot 2 \cdot 2,8$ & - & - & - & - & 2 & - & - & - & - & 233 & 10 & - & - & - & 6 & - & 10 & - & & - & 16 & - & - & - & - & - & - & - & - & 283 & & \\
\hline after $\mathrm{HCl}$ & 1 & - & - & - & 19 & - & - & - & - & - & 28 & - & - & - & - & - & - & - & & - & 167 & 3 & - & - & - & - & - & - & - & 284 & & \\
\hline $47.2-2-4,7$ & 1 & - & - & - & - & - & - & - & - & 186 & 6 & - & - & - & 3 & - & - & - & & - & 36 & - & - & - & - & - & - & - & - & 233 & & \\
\hline after $\mathrm{HCl}$ & 1 & - & - & - & 2 & - & - & - & - & 3 & 27 & - & - & - & - & - & 7 & - & & - & 190 & - & - & 2 & - & - & - & - & - & 242 & & \\
\hline $47.2-2-6,6$ & - & - & - & - & - & - & - & - & - & 277 & 4 & - & - & - & 4 & - & - & - & & - & 30 & - & - & 2 & - & - & - & - & - & 324 & & \\
\hline after $\mathrm{HCl}$ & 2 & 1 & - & - & 1 & - & - & - & - & 51 & 9 & - & - & - & - & - & - & 1 & & - & 154 & 2 & - & 2 & - & - & - & - & - & 264 & & \\
\hline $47,2-2-2,47$ & 10 & - & 9 & $?$ & ? & - & 1 & 22 & - & 1 & 2 & 8 & & 2 & - & - & - & 3 & & - & 173 & 7 & - & - & - & - & 6 & - & 10 & 398 & $\begin{array}{l}4 \mathrm{~V} \\
5^{\mathrm{P}}\end{array}$ & $\underset{3 \mathrm{~K}}{10+14 \mathrm{R}}$ \\
\hline $47.2-3-3.6$ & - & - & - & - & 1 & - & - & - & - & 234 & 14 & - & - & - & 2 & - & - & - & & - & 36 & - & - & - & - & - & - & - & - & 242 & & \\
\hline after $\mathrm{HCl}$ & - & - & - & - & 6 & - & - & - & - & - & 10 & - & - & - & - & - & 6 & - & & - & 191 & 1 & - & 1 & - & - & - & - & - & 231 & & \\
\hline $47.2-4-1,16$ & - & - & - & - & 5 & - & - & - & - & 236 & 1 & - & - & - & 5 & - & - - & - & & - & 7 & - & - & 7 & - & - & 312 & - & - & - & & \\
\hline after $\mathrm{HCl}$ & 1 & - & - & - & 16 & - & - & - & - & 5 & 1 & - & - & - & - & - & 6 & - & & - & 25 & 10 & - & 31 & - & - & 275 & - & - & - & & \\
\hline $47.2-4-3,16$ & 1 & 1 & - & - & 4 & - & - & - & - & 243 & - & - & - & - & 9 & - & - & - & & - & 4 & - & - & 1 & - & 1 & 276 & - & - & - & & \\
\hline after $\mathrm{HCl}$ & - & 1 & 1 & - & 36 & - & - & - & - & 9 & - & - & - & - & 1 & - & 3 & - & & - & - & 78 & - & 8 & - & - & 295 & - & - & - & & \\
\hline $47.2-4-5,16.5$ & 1 & - & - & 2 & 2 & - & - & - & - & 408 & 11 & - & - & - & 7 & - & - & - & & - & 16 & - & 1 & - & - & - & - & - & 4 & 457 & $1 \mathrm{~V}$ & $6^{R}$ \\
\hline after $\mathrm{HCl}$ & 1 & - & 2 & 9 & 9 & - & - & - & - & - & 12 & - & - & - & - & - & - & 1 & & - & 242 & - & 9 & - & - & - & - & - & 10 & 316 & $\begin{array}{l}{ }^{3} \mathrm{~V} \\
1^{\mathrm{P}}\end{array}$ & $26^{R}$ \\
\hline
\end{tabular}


TABLE 3B - Continued

\begin{tabular}{|c|c|c|c|c|c|c|c|c|c|c|c|c|c|c|c|c|c|c|c|c|c|c|c|c|c|c|c|c|c|c|c|c|}
\hline $\begin{array}{c}\text { Sample } \\
\text { Designation }\end{array}$ & 莡 & 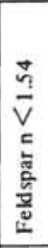 & 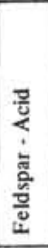 & 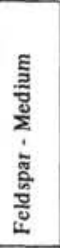 & 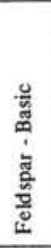 & 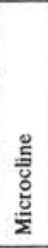 & 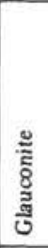 & 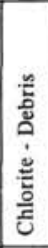 & 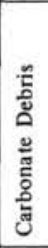 & 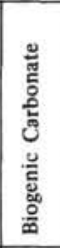 & 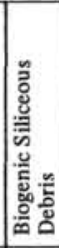 & 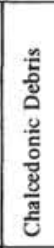 & 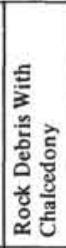 & 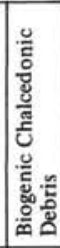 & 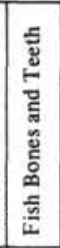 & 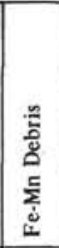 & 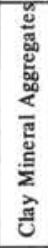 & 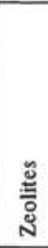 & 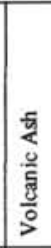 & 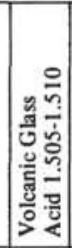 & 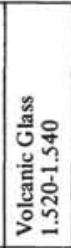 & 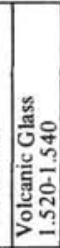 & 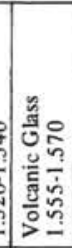 & 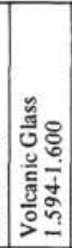 & 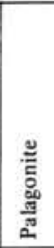 & 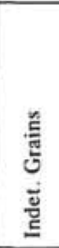 & 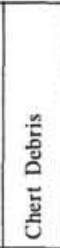 & 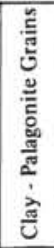 & 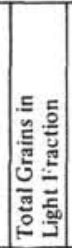 & & "气 & \\
\hline $47 \cdot 2 \cdot 5-1,17$ & 1 & - & 1 & 2 & 2 & - & - & - & - & 239 & 6 & - & - & - & 1 & - & - & - & & - & 116 & - & - & - & - & - & - & - & 1 & 315 & ${ }_{2} \mathrm{~V}$ & ${ }_{6} \mathrm{R}$ \\
\hline after $\mathrm{HCl}$ & 2 & - & 1 & 14 & 14 & - & - & 1 & - & - & 7 & 1 & - & - & - & 1 & - & - & & - & 263 & - & 7 & - & - & - & - & - & 2 & 385 & $? \mathrm{~V}$ & $74 \mathrm{R}$ \\
\hline $47,2-5-3,6$ & - & - & - & 5 & 5 & - & - & - & 2 & 287 & 8 & - & - & - & 5 & - & - & 1 & & - & 58 & - & 1 & - & - & - & - & - & - & 428 & ${ }_{4} \mathrm{~V}$ & $17^{R}$ \\
\hline after $\mathrm{HCl}$ & 1 & - & 2 & 11 & 11 & - & - & - & - & - & 5 & - & - & - & - & - & - & 1 & & - & 335 & - & 7 & - & - & - & - & - & - & 443 & ${ }_{4} \mathrm{~V}$ & ${ }_{77} \mathrm{R}$ \\
\hline $47.2-5-5,6$ & - & - & - & 1 & 1 & - & - & - & - & 280 & 16 & - & - & - & 3 & - & - & - & & - & 103 & - & 2 & - & - & - & - & - & - & 408 & ${ }_{4} \mathrm{R}$ & \\
\hline after $\mathrm{HCl}$ & 1 & - & 1 & 5 & 5 & - & - & - & - & - & 6 & 1 & - & - & - & - & - & - & & - & 414 & - & 4 & - & - & - & - & - & 3 & $?$ & $1^{\mathrm{V}}$ & ${ }_{15} \mathrm{R}$ \\
\hline $47,2-6-2,6$ & - & - & 5 & 8 & 8 & - & - & - & 2 & 282 & 2 & - & - & - & 7 & - & - & - & & - & 222 & - & 2 & - & - & - & - & - & - & 538 & sv & $3^{R}$ \\
\hline after $\mathrm{HCl}$ & - & - & 6 & 8 & 8 & - & - & - & - & - & 2 & - & - & - & - & - & - & - & & - & 362 & - & 5 & - & - & - & - & - & - & 400 & ${ }_{5} \mathrm{~V}$ & $12^{\mathrm{R}}$ \\
\hline $47.2-7-1,17$ & - & - & - & 7 & 7 & - & - & - & - & 313 & 5 & - & - & - & 13 & 4 & - & 1 & & - & 41 & - & - & - & - & - & 3 & - & - & 439 & ${ }_{1}^{2 \mathrm{~V}}$ & $611^{R}$ \\
\hline after $\mathrm{HCl}$ & 2 & - & 8 & 48 & 48 & - & - & - & - & - & 4 & 4 & - & - & - & 3 & - & 6 & & - & 104 & - & 1 & - & - & - & - & - & 7 & 459 & $270^{R}$ & ${ }_{2}^{\mathrm{P}}$ \\
\hline $47.2-7-3,13$ & - & - & - & 1 & 1 & - & - & - & - & 393 & - & 1 & - & - & 118 & - & - & - & & - & 8 & - & - & - & - & - & - & - & - & 523 & $2^{R}$ & \\
\hline $47.2-7-5,27.5$ & 5 & - & 1 & 4 & 4 & - & - & - & - & 220 & 1 & 1 & - & - & 133 & - & - & - & & - & 11 & - & - & - & - & - & - & - & - & 398 & ${ }_{1} \mathrm{~V}$ & $20^{R}$ \\
\hline $47.2-8-1,27$ & 1 & - & 1 & - & - & - & - & - & - & 409 & - & - & - & - & 39 & - & - & 3 & & - & 3 & - & - & - & - & - & - & - & 1 & $?$ & ${ }_{1} \mathrm{~V}$ & ${ }_{2} \mathrm{R}$ \\
\hline $47,2-8-3,18$ & - & - & - & - & - & - & - & - & - & 288 & - & - & - & - & 24 & - & - & - & & - & 3 & - & - & - & - & - & - & - & -1 & 317 & & \\
\hline $47.2-8-5,17$ & 1 & - & - & - & - & - & - & - & - & 318 & - & - & - & - & 126 & - & - & - & & - & 2 & - & - & - & - & - & 2 & - & - & 449 & & \\
\hline $47.2-9 \cdot 1,16$ & - & - & - & - & - & - & - & - & - & 324 & - & - & - & - & 14 & - & - & - & & - & 1 & - & - & - & - & - & - & - & - & 339 & & \\
\hline $47.2-9-3,17$ & 1 & - & - & - & - & - & - & - & - & 212 & - & - & - & - & 46 & - & - & 1 & & - & 2 & - & - & - & - & - & 5 & - & - & 267 & & \\
\hline $47.2-10-2,17$ & - & - & - & - & - & - & - & - & - & 249 & - & - & - & - & 44 & - & - & - & & - & 3 & - & - & - & - & - & 3 & - & - & 299 & & \\
\hline $47.2-10-4,17$ & 2 & 1 & - & - & - & - & - & - & - & 267 & - & - & - & - & 65 & - & - & - & & - & $?$ & - & - & - & - & - & 3 & - & - & 339 & & \\
\hline $47.2-10-6,16$ & 2 & 1 & - & - & 2 & - & - & - & - & 247 & - & 1 & - & - & 18 & 1 & - & - & & - & 2 & - & - & - & - & - & 3 & - & - & 277 & & \\
\hline $47.2-11-2,145$ & - & - & - & - & - & - & - & 1 & - & 300 & - & - & - & - & - & - & - & - & & - & - & - & - & - & - & - & - & - & - & 301 & & \\
\hline after $\mathrm{HCl}$ & 3 & - & - & - & - & - & - & 99 & - & - & 1 & 26 & - & - & - & 23 & - & 3 & & - & 51 & - & - & - & - & - & - & - & 25 & 243 & $2^{2} \mathrm{~V}$ & $5^{\mathrm{R}}$ \\
\hline $47.2-12-2,17$ & - & - & - & - & - & - & - & - & - & 256 & - & 112 & - & - & 8 & - & - & - & & - & 7 & - & - & - & - & - & - & - & - & 353 & ${ }^{2 R}$ & 70 \\
\hline after $\mathrm{HCl}$ & - & - & - & 1 & 1 & - & - & 1 & - & - & - & 267 & - & - & - & 1 & - & - & & - & 79 & - & - & - & - & - & 4 & - & - & 369 & $\begin{array}{r}1 \mathrm{~V} \\
110\end{array}$ & ${ }_{4} \mathrm{R}$ \\
\hline $47.2-12-4,145$ & 1 & - & - & - & - & - & - & - & - & 265 & - & 4 & - & - & 21 & 2 & - & - & & - & 4 & - & - & - & - & - & - & - & - & 300 & & \\
\hline $47.2-13-2,145$ & 2 & - & 1 & - & - & - & - & - & - & 344 & - & 10 & - & - & 12 & 1 & - & - & & - & 1 & - & - & - & - & - & - & - & - & 371 & & \\
\hline $47.2-13-6,145$ & 1 & - & - & - & - & - & - & - & - & 283 & - & 22 & - & - & 12 & - & - & - & & - & - & - & - & - & - & - & - & - & - & 318 & & \\
\hline after $\mathrm{HCl}$ & - & - & - & - & 1 & - & - & - & - & 28 & - & 215 & - & - & - & - & - & 1 & & - & 4 & - & - & - & - & 1 & 6 & - & - & 256 & & \\
\hline $47.2-14-3,145$ & 17 & 5 & 8 & - & 3 & - & - & - & - & 8 & 4 & $?$ & 4 & - & - & - & 4 & - & & - & 14 & - & 3 & ? & - & - & 6 & 27 & - & $?$ & & \\
\hline $47.2-14-4,145$ & - & - & - & - & - & - & - & - & - & 278 & - & - & - & - & 4 & 2 & - & - & & - & 2 & - & - & - & - & - & 286 & - & - & - & & \\
\hline after $\mathrm{HCl}$ & - & - & - & - & - & - & - & - & - & 18 & - & - & - & - & - & 4 & - & - & & - & 1 & - & - & - & - & - & - & - & - & - & & \\
\hline
\end{tabular}


TABLE 3B - Continued

\begin{tabular}{|c|c|c|c|c|c|c|c|c|c|c|c|c|c|c|c|c|c|c|c|c|c|c|c|c|c|c|c|c|c|c|c|c|}
\hline $\begin{array}{c}\text { Sample } \\
\text { Designation }\end{array}$ & 菢 & 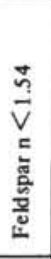 & 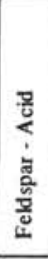 & 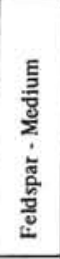 & 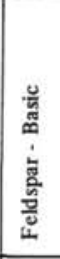 & 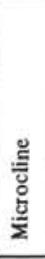 & 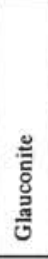 & 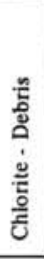 & 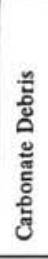 & 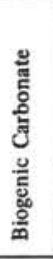 & 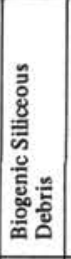 & 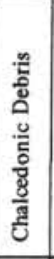 & 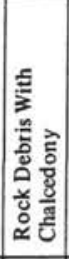 & 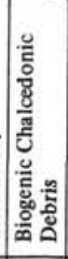 & 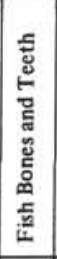 & 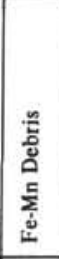 & 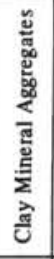 & 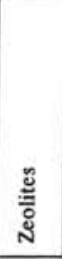 & $\begin{array}{l}\frac{5}{3} \\
\frac{5}{5} \\
\frac{0}{5} \\
\frac{3}{0} \\
>\end{array}$ & 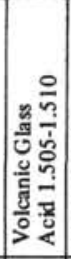 & 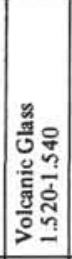 & 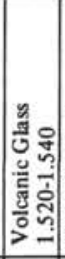 & 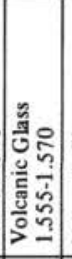 & 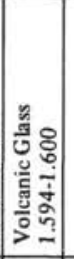 & 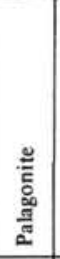 & 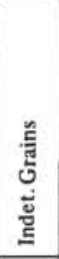 & 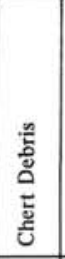 & 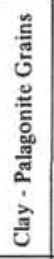 & 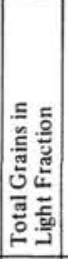 & \multicolumn{3}{|c|}{ "气 } \\
\hline $50.1-4-1,75$ & 2 & - & 4 & 5 & & & & & & - & - & - & 2 & 1 & 19 & 21 & & 153 & & & 1 & & - & & & - & - & & 95 & 314 & ${ }_{2 \mathrm{P}}^{\mathrm{V}}$ & $3^{R}$ \\
\hline $51.0-1-2$ & 4 & - & 6 & 23 & 23 & - & - & - & 2 & - & 3 & 9 & 1 & 2 & 11 & 28 & & 3 & & & 135 & 18 & & & & - & 4 & & 113 & 459 & ${ }_{2}^{19 \mathrm{~V}}$ & $84 \mathrm{R}$ \\
\hline $51.0-1-4,6$ & 10 & 1 & 2 & & 12 & - & & - & & 1 & 1 & - & - & - & 20 & - & 6 & - & & & 28 & - & 1 & - & - & - & - & - & & 235 & & \\
\hline $51.0-1-6,16$ & 2 & 1 & - & & - & - & & - & & 1 & 4 & - & - & - & 279 & 4 & 7 & - & & & 2 & - & 1 & - & - & - & - & - & & 308 & & \\
\hline $51.1-1-4$ & 1 & - & 21 & 37 & 37 & & & & & - & - & 2 & 2 & - & 1 & - & & 1 & & & 33 & & - & & & - & - & & 2 & 313 & ${ }_{2}^{1 \mathrm{M}}$ & $\begin{array}{r}8 \mathrm{R} \\
63^{\mathrm{K}}\end{array}$ \\
\hline $51.1 \cdot 2-2$ & - & - & 1 & 3 & 3 & & & & & - & - & 1 & 1 & 6 & 291 & 2 & & 2 & & & 13 & & 3 & & & 1 & - & & 4 & 242 & ${ }_{1} \mathrm{~V}$ & ${ }_{2} \mathrm{P}$ \\
\hline $52.0-1 \cdot 2$ & - & - & 4 & 8 & 8 & & & & & - & 1 & 1 & - & - & 1 & 1 & & - & & & 19 & & 2 & & & - & - & & 7 & 352 & $1 \mathrm{~A}$ & $17 \mathrm{R}$ \\
\hline $52.0-2-5$ & 11 & 1 & 7 & 112 & 112 & & & & & - & 2 & - & - & 2 & 1 & 7 & & 3 & & & 103 & & 2 & & & - & 40 & & 5 & 396 & $134^{R}$ & ${ }_{2} \mathrm{P}$ \\
\hline $52.0-3-5$ & - & 6 & 8 & 180 & 180 & & & & & - & 1 & - & 1 & 1 & - & - & & 4 & & & 2 & & - & & & 1 & 27 & & 3 & 293 & ${ }_{65} \mathrm{R}$ & ${ }_{2} \mathrm{P}$ \\
\hline $52.0-4-2$ & 2 & 2 & 16 & 138 & 138 & - & - & - & - & - & 2 & 4 & - & - & - & 53 & & 13 & & & 6 & 1 & & & & 1 & 139 & & 3 & 480 & $72 \mathrm{R}$ & ${ }_{2} \mathrm{P}$ \\
\hline $52.0-5-2$ & - & - & 34 & 81 & 81 & & & & & - & - & 1 & 1 & 1 & 1 & 42 & 8 & & & & 4 & & 1 & & & & 112 & & 10 & 336 & $36^{\mathrm{R}}$ & ${ }_{1} \mathrm{P}$ \\
\hline $52.0-5-3$ & 2 & - & 41 & 110 & 110 & - & - & - & - & - & 2 & 1 & - & - & 5 & 50 & & 9 & & & 2 & - & & & & 1 & 36 & & & 332 & $\begin{array}{l}3 \mathrm{~V} \\
1^{\mathrm{P}}\end{array}$ & ${ }_{24} \mathrm{R}$ \\
\hline $52.0-8-2$ & - & & 1 & 2 & 2 & & & & & - & - & 1 & 184 & 103 & 340 & 2 & & 1 & & & 1 & & - & & & - & - & & 1 & 406 & $1^{V}$ & ${ }_{1} \mathrm{P}$ \\
\hline $52.0-8-4$ & - & - & 41 & 110 & 110 & - & & - & - & - & - & 36 & - & 32 & 3 & 3 & & - & & & 1 & 1 & & & & - & - & & 6 & 425 & & \\
\hline $52.0-8-6$ & - & - & - & 1 & 1 & & & & & - & - & - & 186 & 54 & 226 & 2 & & 2 & & & 1 & & - & & & 4 & 2 & & 3 & 326 & $2^{\mathrm{R}}$ & \\
\hline $53.0-0-2$ & 10 & 3 & 2 & 15 & 15 & - & 1 & - & 1 & 3 & - & - & - & - & - & - & & - & & 132 & 9 & & 2 & - & & - & 2 & & - & 204 & $11^{\mathrm{V}}$ & $11^{R}$ \\
\hline $53.0-1-1$ & - & - & 4 & 13 & 13 & & & & - & - & 1 & & 4 & 2 & - & - & & 1 & & & 46 & & 48 & & & - & 6 & & - & 484 & $\begin{array}{r}{ }_{1}^{97 \mathrm{~V}} \\
\mathrm{P}\end{array}$ & $201^{R}$ \\
\hline $53.0-7-1$ & - & - & 35 & 90 & 90 & - & - & - & - & - & - & - & - & - & 3 & - & & 1 & & & 2 & - & & & & - & 8 & & - & 336 & ${ }_{2}^{1 \mathrm{P}}$ & $193^{R}$ \\
\hline $53.1-1-1$ & 9 & - & 6 & 138 & 138 & - & - & & & - & - & 5 & - & 3 & 1 & - & & 2 & & & 2 & - & & & & - & 43 & & 12 & 264 & $\begin{array}{l}{ }_{3}^{3} \mathrm{P} \\
{ }^{\mathrm{P}}\end{array}$ & $40^{\mathrm{R}}$ \\
\hline $53 \cdot 1-1-2$ & 1 & - & $?$ & 82 & 82 & - & - & - & - & - & 1 & 44 & 26 & 66 & 5 & 2 & & 1 & & & 2 & 1 & & & & - & 30 & & - & 287 & $14 \mathrm{R}$ & ${ }_{1} \mathrm{P}$ \\
\hline $53.1-1-4,11$ & 6 & - & 4 & & 151 & - & & 3 & & - & - & 11 & - & - & 1 & - & - & - & & - & - & - & - & - & - & - & s5 & - & & 282 & & \\
\hline $53.1-1-6,28$ & 1 & - & 1 & & 112 & - & & - & & - & 3 & - & - & 2 & 10 & 12 & 99 & 2 & & & 1 & - & 1 & 6 & - & - & 3 & - & & 297 & & \\
\hline $53.1-2-4$ & 2 & - & $?$ & 67 & 67 & - & - & - & - & - & - & - & - & 1 & & 1 & & - & & & 35 & 15 & & & & - & - & & - & 548 & $\begin{array}{r}18 \mathrm{~V} \\
{ }_{3}^{\mathrm{P}}\end{array}$ & $502^{R}$ \\
\hline $53,1 \cdot 2-3,16$ & - & 1 & - & & 38 & - & & - & & - & 1 & 1 & - & - & - & - & 6 & - & & & 141 & - & 12 & 5 & - & 2 & 12 & - & & 315 & & \\
\hline $53.1-2-6$ & - & - & $?$ & 42 & 42 & - & - & - & - & - & 2 & 1 & - & & 1 & - & & - & & & 48 & 31 & & & & - & - & & - & 454 & $\begin{array}{l}{ }_{1}^{88 \mathrm{~V}} \\
\mathrm{P}\end{array}$ & $230^{R}$ \\
\hline
\end{tabular}


TABLE 3B - Continued

\begin{tabular}{|c|c|c|c|c|c|c|c|c|c|c|c|c|c|c|c|c|c|c|c|c|c|c|c|c|c|c|c|c|c|c|c|c|}
\hline $\begin{array}{c}\text { Sample } \\
\text { Designation }\end{array}$ & $\begin{array}{l}\tilde{\tau} \\
\tilde{z} \\
\tilde{g}\end{array}$ & 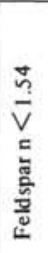 & 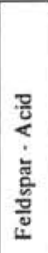 & 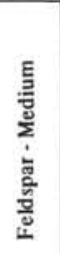 & 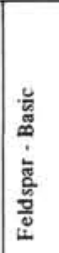 & $\begin{array}{l}\frac{0}{E} \\
\frac{\tilde{E}}{0} \\
\frac{\tilde{g}}{\Sigma}\end{array}$ & 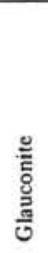 & 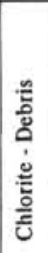 & 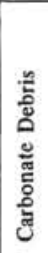 & 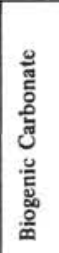 & 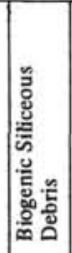 & 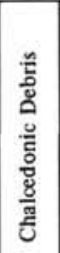 & 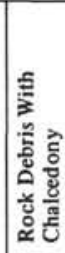 & 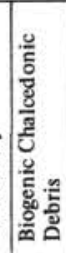 & 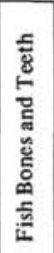 & 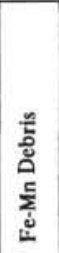 & 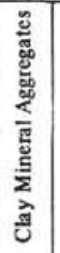 & 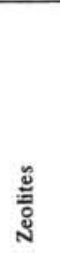 & 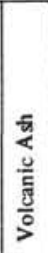 & 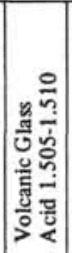 & 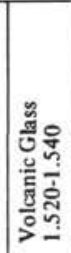 & 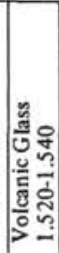 & 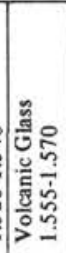 & 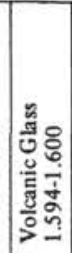 & 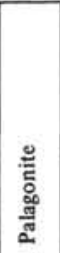 & 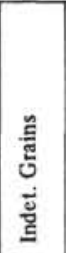 & 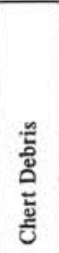 & 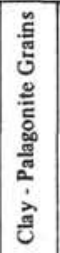 & 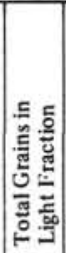 & \multicolumn{3}{|c|}{ ఊొ } \\
\hline $53.1-3-3,23$ & 3 & - & - & & 40 & - & & - & & - & 1 & 1 & - & 1 & - & - & - & - & & & - & 31 & 16 & 58 & - & - & 1 & - & & 294 & & \\
\hline $53.1-3-5$ & - & - & - & 51 & 51 & - & - & - & - & - & - & - & - & - & 1 & - & & 1 & & & 43 & 12 & & & & - & - & & - & 403 & ${ }_{2}^{100 \mathrm{~V}}$ & $370^{R}$ \\
\hline $53.2-1-1$ & 2 & - & $?$ & 140 & 140 & - & - & - & - & - & - & - & - & - & 2 & - & & 1 & & & 8 & 3 & & & & - & 19 & & - & & ${ }_{1}^{23} \mathrm{P}$ & $32^{R}$ \\
\hline $53.2-1-3,23$ & 1 & 1 & 1 & & 64 & - & & - & & - & - & - & - & - & - & - & - & - & & & - & 15 & 4 & 7 & - & - & - & - & & 254 & & \\
\hline $53.2-1-5,7$ & - & - & 1 & & 49 & - & & - & & - & - & - & - & - & - & - & & - & & & - & 56 & 6 & 19 & - & - & 14 & - & & 340 & & \\
\hline $53.0-6-2,66$ & 3 & so & - & & - & - & & - & & 1 & 1 & - & - & - & 2 & - & - & - & & & - & 17 & 1 & 1 & - & - & 41 & - & & 343 & & \\
\hline $54.0-1-1$ & - & - & $?$ & 16 & 16 & - & - & - & - & - & 2 & - & - & - & 1 & - & & - & & & 229 & 47 & 47 & & & - & 2 & & - & 399 & $18 \mathrm{~V}$ & $32 \mathrm{R}$ \\
\hline $54.0-1-3,35$ & 1 & - & - & & 8 & - & & - & & - & 3 & 1 & - & - & - & - & 2 & - & & & - & 247 & 24 & 10 & - & - & - & - & & 337 & & \\
\hline $54.0-2-2$ & 1 & - & $?$ & 77 & 77 & - & - & - & - & 1 & - & - & 1 & - & 1 & - & & - & & & 44 & 2 & & & & - & 3 & & - & 416 & $534 \mathrm{R}$ & ${ }_{3} \mathrm{P}$ \\
\hline $54.0-4-1$ & - & - & - & 5 & 5 & - & - & - & - & - & 3 & - & - & - & - & - & & - & & & 418 & 11 & & & & - & - & & - & 454 & $61^{R}$ & ${ }_{2} \mathrm{P}$ \\
\hline $54 \cdot 0-7 \cdot 2$ & 1 & - & $?$ & 39 & 39 & - & - & - & - & - & 3 & - & - & - & 3 & 21 & & & & & 124 & 29 & & & & 1 & - & & - & 369 & $\begin{array}{r}13 \mathrm{~V} \\
{ }_{2} \mathrm{P}\end{array}$ & $267^{R}$ \\
\hline $54.0-6-2,16$ & - & 1 & - & & 4 & - & & - & & - & - & - & - & - & - & - & - & - & & - & 233 & 1 & 9 & - & - & - & - & - & & 216 & & \\
\hline $54,0-6-4,16$ & 1 & - & - & & 32 & - & & 1 & & - & 1 & 1 & - & - & - & - & - & - & & 94 & - & 137 & 12 & 4 & - & 2 & - & & - & 285 & & \\
\hline $54.0-6-5,17$ & - & - & - & & 19 & - & & - & & - & 1 & - & - & - & - & - & - & - & & 71 & - & 184 & 9 & 7 & - & 1 & - & & - & 292 & & \\
\hline $55.0-1 \cdot 2$ & 11 & 1 & 3 & & 7 & - & & - & & 12 & 46 & 4 & - & - & 8 & - & 33 & - & & 83 & 41 & - & 3 & 5 & - & 2 & - & & - & 259 & & \\
\hline $55.0-2-5,7$ & 1 & - & - & & 4 & & & - & & 236 & 2 & & & & 8 & - & - & - & & & - & 44 & & 3 & - & - & - & & & 333 & & \\
\hline $55.0-2-6$ & 1 & - & 2 & 4 & 4 & - & - & - & - & 321 & 3 & 1 & - & 2 & 2 & - & & - & & - & 46 & & 5 & - & & - & - & & - & 404 & ${ }_{1}^{s \mathrm{~V}}$ & $10^{R}$ \\
\hline $55.0-4-2,145$ & 2 & - & - & & 12 & & & 3 & & 192 & - & & & & 18 & & - & 1 & & & - & 53 & & 5 & - & 1 & - & & & 314 & & \\
\hline $55.0-8-1$ & 2 & - & 2 & 18 & 18 & 1 & - & 2 & - & 99 & 152 & - & - & 2 & 2 & 3 & & - & & - & 30 & & 6 & - & & - & - & & & 383 & $\begin{array}{l}{ }_{2}^{6} \mathrm{P} \\
\end{array}$ & $62^{R}$ \\
\hline $55.0-8-5,18$ & - & 5 & 1 & & 3 & & & - & & 2 & 178 & & & & - & 11 & 7 & - & & & 72 & 5 & & 3 & - & 2 & - & & & 314 & & \\
\hline $55.0-10-3$ & 1 & - & - & 2 & 2 & - & - & - & - & - & 305 & - & - & - & - & - & 3 & - & & - & 12 & & - & - & & - & - & & 4 & 418 & $\begin{array}{l}{ }_{1}^{4 V} \\
1^{P}\end{array}$ & ${ }_{11} R^{R}$ \\
\hline $55.0-11-1,46$ & 1 & - & 1 & & 5 & & & - & & - & 193 & & & & - & & & - & & & - & 24 & & 6 & - & - & - & & & 297 & & \\
\hline $55.0-11-5$ & 1 & - & - & 1 & 1 & - & - & - & - & - & 341 & - & - & - & - & 3 & & - & & - & 5 & & 2 & - & & - & - & & 1 & 407 & $3^{R}$ & \\
\hline $55.0-12-1$ & 3 & 1 & 4 & 15 & 15 & - & - & - & - & 1 & 31 & - & - & I & - & - & & 1 & & - & 63 & & 5 & 1 & & - & - & & - & 331 & $\underset{1}{22}{ }_{1}^{2}$ & $182^{R}$ \\
\hline $55.0-12-5,7$ & - & 1 & - & & 84 & & & 3 & & - & 43 & & & & - & - & 3 & - & & & & 13 & & 16 & - & 8 & 7 & & & 348 & & \\
\hline $55.0-13-3,7$ & 1 & - & - & & 16 & & & - & & 2 & 127 & & & & - & 4 & 7 & - & & & - & 19 & & 2 & - & 5 & - & & & 236 & & \\
\hline $55.0-14-1$ & 1 & 3 & - & 3 & 3 & - & - & 8 & - & 2 & 27 & 1 & - & - & - & 2 & & - & & - & 168 & & 6 & 1 & & - & - & & - & 289 & ${ }_{8}^{9 \mathrm{P}}$ & $269 \mathrm{R}$ \\
\hline
\end{tabular}


TABLE 3B - Continued

\begin{tabular}{|c|c|c|c|c|c|c|c|c|c|c|c|c|c|c|c|c|c|c|c|c|c|c|c|c|c|c|c|c|c|c|c|c|}
\hline $\begin{array}{c}\text { Sample } \\
\text { Designation }\end{array}$ & $\frac{N}{2}$ & 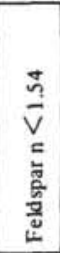 & 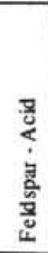 & 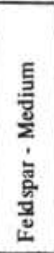 & 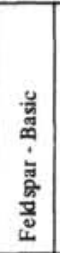 & 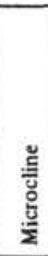 & 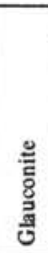 & 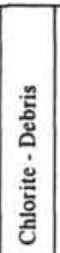 & 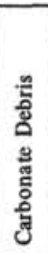 & 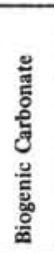 & 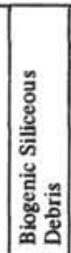 & 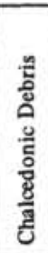 & 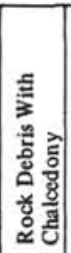 & 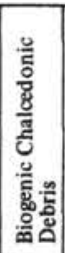 & 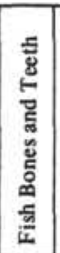 & 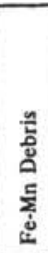 & 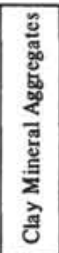 & 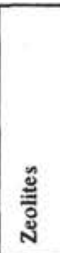 & $\begin{array}{l}\frac{5}{2} \\
0 \\
\frac{0}{5} \\
\frac{8}{0} \\
>\end{array}$ & 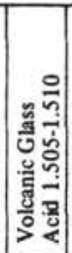 & 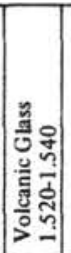 & 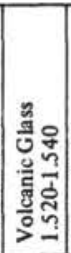 & 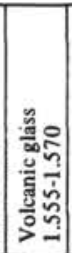 & 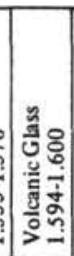 & 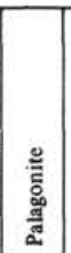 & 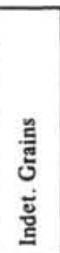 & 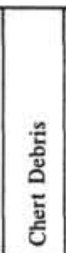 & 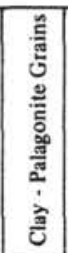 & 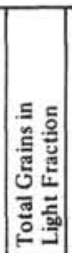 & & 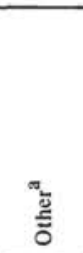 & \\
\hline $55.0-14-3.145$ & - & 2 & - & & 4 & & & - & & - & 30 & & & & - & 1 & - & - & & & - & 144 & & 5 & - & 2 & - & & & 231 & & \\
\hline $55.0-14-6$ & 1 & - & 1 & 2 & 2 & - & - & 16 & - & 4 & 108 & - & - & - & - & - & & - & & - & 233 & & 7 & 1 & & - & - & & - & 413 & $\begin{array}{r}1 \mathrm{M} \\
181^{\mathrm{R}}\end{array}$ & ${ }_{5}^{8 \mathrm{P}}$ \\
\hline $56.2 \cdot 1-2$ & 2 & - & 3 & 18 & 18 & - & - & & & 1 & 8 & 1 & & 1 & - & - & & 2 & & & & 2 & & - & & 4 & 5 & & - & 424 & $\begin{array}{r}326 \mathrm{~V} \\
{ }_{1} \mathrm{~V}\end{array}$ & $200^{R}$ \\
\hline $56.2-1 \cdot 4$ & 6 & 1 & $?$ & & & 1 & - & - & - & 1 & 9 & - & 22 & - & - & 2 & & 3 & & & 1 & - & & & & - & - & & 5 & 294 & $\begin{array}{r}162 \mathrm{~V} \\
1 \mathrm{O}\end{array}$ & $50^{R}$ \\
\hline $56.2-2-2.90$ & 21 & 5 & 2 & & 3 & - & & - & & - & 181 & - & & - & - & 1 & - & - & & 10 & 10 & - & & - & 16 & - & & & 2 & 251 & & \\
\hline $56.2-2-6,16$ & 11 & 3 & 1 & & 4 & - & & - & & 1 & 126 & - & - & - & - & - & 10 & - & & 23 & 4 & - & - & 14 & - & - & - & & - & 197 & & \\
\hline $56.2-3-2,7$ & 3 & 39 & - & 12 & - & - & & - & & 2 & 28 & 1 & & - & - & - & - & - & & 182 & & so & & 2 & - & - & - & & - & 319 & & \\
\hline $56.2-3-2,145$ & 12 & 2 & 1 & 14 & 1 & - & & 1.3 & & 35 & 229 & - & & - & - & - & - & - & & 3 & & - & & - & - & - & - & & - & 316 & & \\
\hline $56.2-4-2,10$ & 7 & 1 & - & & 1 & - & & - & & 8 & 145 & - & - & - & - & 1 & 3 & - & & 21 & 10 & - & 1 & 6 & - & 2 & - & & - & 206 & & \\
\hline $56.2-4-5,13$ & 10 & 2 & 2 & & 15 & - & & 5 & & 2 & 182 & - & & - & - & - & 7 & - & & 50 & - & 30 & & 3 & - & - & - & & - & 308 & & \\
\hline $56.2-6-4$ & 3 & 1 & $?$ & 19 & 19 & & - & - & - & 1 & 88 & - & - & - & 1 & 2 & & 1 & & & 83 & 4 & & & & - & - & & 16 & 353 & ${ }_{4}^{5 \mathrm{~V}}$ & $396^{R}$ \\
\hline $56.2-6-6,16$ & 2 & 1 & 5 & & 18 & 1 & & - & & - & 3 & - & & - & - & - & - & - & & 253 & - & 5 & & - & 8 & - & - & & - & 296 & & \\
\hline $56.2 \cdot 7 \cdot 2,43$ & 4 & 2 & - & & 1 & - & & - & & 5 & 31 & - & & - & - & - & - & - & & 52 & -1 & 204 & & 2 & - & - & - & & - & 301 & & \\
\hline $56.2-7-4,39$ & 6 & 21 & 9 & & 21 & - & & 1 & & 4 & 75 & - & & - & 1 & - & - & - & & 95 & - & 42 & & 2 & - & - & - & & 3 & 280 & & \\
\hline $56.2 \cdot 8 \cdot 2,7$ & 2 & 3 & 1 & & 3 & 1 & & - & & - & 62 & - & - & - & 1 & - & - & - & & 142 & - & 63 & 2 & 11 & - & - & - & & - & 291 & & \\
\hline $56.2-9-6,8$ & - & 2 & 2 & & 19 & - & & - & & - & 23 & - & & - & - & - & - & - & & 176 & - & 32 & & 3 & - & - & - & & 14 & 271 & & \\
\hline $56.2-10-4$ & 3 & 1 & 3 & 10 & 10 & - & 1 & & & 1 & 50 & - & 1 & 1 & 1 & 1 & & - & & & 93 & & - & & & 4 & - & & 9 & 342 & $\begin{array}{r}16 \mathrm{~V} \\
{ }_{4} \mathrm{P}\end{array}$ & $462^{R}$ \\
\hline $56.2-5-4$ & 6 & - & 5 & 11 & 11 & - & - & & & - & 218 & 3 & 1 & - & - & - & & - & & & 19 & & 1 & & & 7 & - & & 3 & 322 & ${ }_{4} \mathrm{~V}$ & $49 \mathrm{R}$ \\
\hline $57.1-1-2,0$ & 1 & 1 & - & & 20 & - & & - & & - & 84 & - & & - & - & - & - & - & & 78 & - & 19 & & - & 77 & - & - & & - & 281 & & \\
\hline $57.1-1-3$ & - & - & 1 & 12 & 12 & - & - & & & 1 & 44 & - & 2 & 1 & - & - & & 2 & & & 12 & & 1 & & & 14 & - & & - & 340 & & \\
\hline $57.1-1-5,7$ & 7 & 6 & 2 & & 7 & - & & - & & - & 75 & - & & - & - & - & 20 & 1 & & 57 & - & 17 & & - & 21 & 1 & - & & - & 214 & & \\
\hline $57.1-1-8,7$ & 4 & 3 & 1 & & 4 & - & & - & & 35 & 53 & - & - & - & - & 2 & - & - & & 103 & - & 17 & - & - & 82 & - & - & & - & 338 & & \\
\hline $57,1-2-1,44$ & 1 & 1 & - & & 16 & - & & - & & - & 13 & 2 & - & - & - & - & - & - & & 87 & - & 7 & 3 & - & 191 & - & - & & - & 321 & & \\
\hline $57.1-3-2,108$ & - & 1 & - & & 9 & - & & - & & - & 7 & 2 & - & - & - & - & - & - & & 75 & - & 1 & 2 & - & 135 & 1 & - & & - & 233 & & \\
\hline $57.2-1-1$ & 11 & 2 & $?$ & 5 & 5 & - & - & & 1 & 3 & 164 & 3 & - & - & 4 & - & & - & & & 57 & 5 & & & & - & - & & 13 & 236 & & \\
\hline $58.1-3,145$ & 1 & 3 & - & & 24 & - & & - & & - & 17 & 1 & & - & - & - & - & 21 & & 85 & - & 12 & & - & 38 & 34 & - & & - & 236 & & \\
\hline $58.2-1-3,16$ & - & 7 & 3 & & 7 & - & & - & & 55 & 50 & - & & - & 3 & - & 22 & 2 & & 69 & - & 52 & & - & 15 & 36 & 10 & & - & 331 & & \\
\hline $58.2-1-5$ & 1 & 1 & 14 & 9 & 9 & - & - & & & 1 & 71 & - & 1 & - & - & - & & - & & & 20 & & 2 & & & 8 & 22 & & - & 301 & $\begin{array}{l}13 \mathrm{~V} \\
{ }_{2}^{\mathrm{P}}\end{array}$ & $244 R$ \\
\hline
\end{tabular}




\begin{tabular}{|c|c|c|c|c|c|c|c|c|c|c|c|c|c|c|c|c|c|c|c|c|c|c|c|c|c|c|c|c|c|c|c|c|}
\hline $\begin{array}{c}\text { Sample } \\
\text { Designation }\end{array}$ & $\frac{\mathrm{N}}{\mathrm{g}}$ & 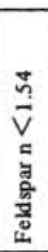 & 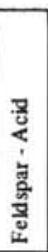 & 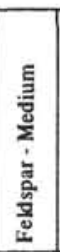 & 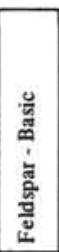 & 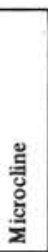 & 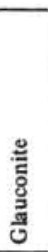 & 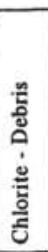 & 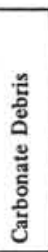 & 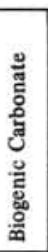 & 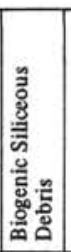 & 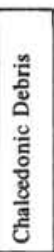 & 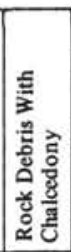 & 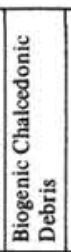 & 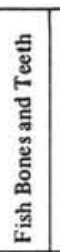 & 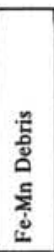 & 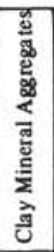 & 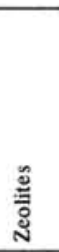 & 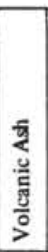 & 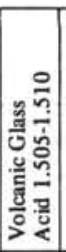 & 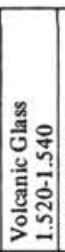 & 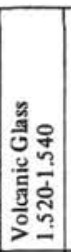 & 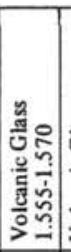 & 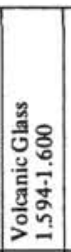 & 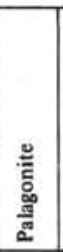 & 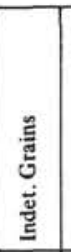 & 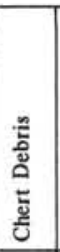 & 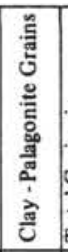 & 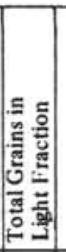 & \multicolumn{3}{|c|}{ "ू } \\
\hline $59.1-3-2,11$ & - & - & - & & 2 & - & & - & & - & 7 & - & & - & 2 & 2 & 65 & 57 & & - & - & 3 & & - & 1 & 25 & 38 & & - & 272 & & \\
\hline $59.1-3-5,146$ & 2 & 3 & 4 & & 9 & - & & - & & 5 & 2 & - & & - & 5 & 4 & 22 & 13 & & - & - & 13 & & - & 2 & 60 & 25 & & - & 169 & & \\
\hline $59.2-1-2,4$ & 1 & 2 & 1 & & 6 & - & & 6 & & 2 & 61 & - & & - & 4 & 5 & - & - & & 47 & 60 & 4 & & 1 & - & 17 & 8 & & 121 & 346 & & \\
\hline $59.2-2-3$ & 1 & - & 3 & 14 & 14 & - & - & & & 1 & 59 & - & 4 & 1 & 2 & 2 & & 2 & & & 79 & & - & & & 9 & - & & 143 & 422 & $\begin{array}{l}4 \mathrm{~V} \\
{ }_{8}^{\mathrm{P}}\end{array}$ & $379 R$ \\
\hline $59.2-2-5,35$ & - & 1 & - & & 6 & - & & - & & 3 & 3 & - & & - & 1 & - & - & - & & 112 & 133 & 7 & & - & 12 & 5 & - & & - & 283 & & \\
\hline $59.2-3-2$ & 1 & - & 8 & 84 & 84 & - & - & & & 1 & 8 & 24 & - & - & 13 & 14 & & 22 & & & 4 & & - & & & 10 & - & & 106 & 392 & $\underset{6^{P}}{11 \mathrm{~V}}$ & $151^{R}$ \\
\hline $60.0-1-1,7$ & 1 & 1 & - & & 34 & - & & - & & 13 & 7 & - & & 3 & - & - & 10 & 5 & & & - & 12 & 1 & - & - & 134 & - & & - & 263 & & \\
\hline $60.0-2-1$ & - & - & - & 164 & 164 & - & 1 & & & 4 & 2 & - & - & - & - & - & & 4 & & & 83 & & 11 & & & 18 & - & & 2 & 335 & $21 \mathrm{~V}$ & $68 \mathrm{R}$ \\
\hline $60.0-3-2,8$ & 2 & 3 & 2 & & 74 & - & & - & & 7 & 4 & - & & 4 & - & - & - & 1 & & & - & 27 & 10 & 35 & - & 4 & - & & - & 259 & & \\
\hline $60.0-5-1,51$ & - & - & - & & 20 & - & & 5 & & 15 & 3 & 1 & & - & - & - & - & - & & & - & 28 & 4 & 2 & - & 2 & - & & - & 290 & & \\
\hline $60.0-5-3,40$ & 1 & - & 1 & & 29 & - & & - & & 2 & 1 & - & & - & - & - & - & - & & & 195 & - & 6 & 25 & - & - & - & & - & 315 & & \\
\hline $60.0-6-2$ & 1 & 1 & 4 & 20 & 20 & 1 & - & & & & 40 & - & - & 6 & 5 & 4 & & 1 & & & 84 & & 1 & & & 12 & - & & 107 & 368 & ${ }_{4 \mathrm{P}}^{2 \mathrm{~V}}$ & $111^{R}$ \\
\hline $60.0-6-4,25$ & - & - & 1 & & 42 & - & & - & & 2 & 3 & - & & - & - & - & - & - & & & 124 & - & 2 & - & 31 & 2 & - & & - & 338 & & \\
\hline $60.0-6-6$ & - & - & 2 & 34 & 34 & - & - & & & 2 & 1 & - & - & - & - & - & & - & & & 232 & & 1 & & & 2 & - & & - & 517 & $\begin{array}{l}78 \mathrm{~V} \\
1^{\mathrm{P}}\end{array}$ & $234^{R}$ \\
\hline $60.0-8-1$ & 1 & - & 2 & 44 & 44 & - & - & & & 1 & 1 & 121 & - & - & - & - & & 17 & & & 2 & & 2 & & & 1 & - & & 2 & 339 & $\begin{array}{r}131^{\mathrm{V}} \\
{ }_{2} \mathrm{P}\end{array}$ & $\begin{array}{r}74 \mathrm{R} \\
{ }_{1} \mathrm{O}\end{array}$ \\
\hline $60.0-8-3,145$ & - & 1 & - & & 36 & - & & - & & - & 3 & - & & 141 & - & - & - & 8 & & & - & 64 & 15 & - & 13 & 1 & - & & - & 393 & & \\
\hline $60.0-9-5$ & - & - & $?$ & 164 & 164 & - & - & & - & 1 & 1 & - & 51 & - & 1 & - & & 3 & & & 21 & 2 & & & & - & - & & 9 & 348 & ${ }_{5} \mathrm{~V}$ & $87 \mathrm{R}$ \\
\hline $60.03-\mathrm{CC}$ & 4 & - & 2 & & 4 & & & - & & 9 & - & & & & & - & - & - & & & 3 & - & & 6 & - & - & - & & & 271 & & \\
\hline
\end{tabular}

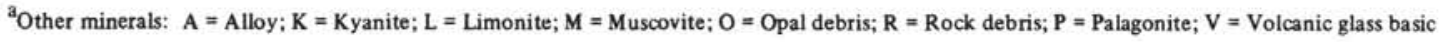


TABLE 4A

Mineralogical Analysis of Heavy Fraction in Size Range $0.05-0.01 \mathrm{~mm}$

\begin{tabular}{|c|c|c|c|c|c|c|c|c|c|c|c|c|c|c|c|c|c|c|c|c|c|c|c|c|c|c|c|c|c|c|c|}
\hline $\begin{array}{c}\text { Sample } \\
\text { Designation }\end{array}$ & 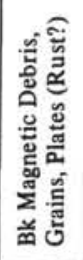 & 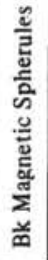 & 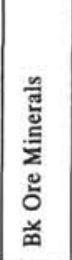 & 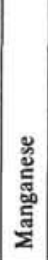 & 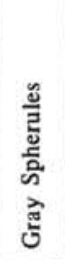 & $\overrightarrow{\underline{z}}$ & 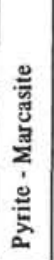 & 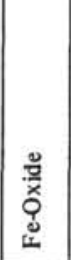 & 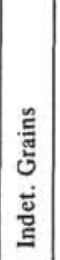 & 导 & 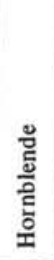 & 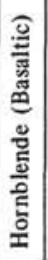 & 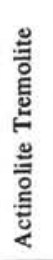 & 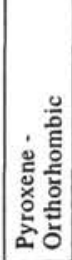 & 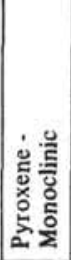 & 营 & 总 & 芯 & $\frac{\stackrel{0}{0}}{\frac{0}{0}}$ & 气 & 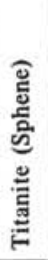 & 总 & 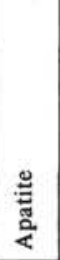 & 를 & है & 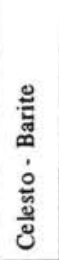 & 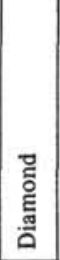 & 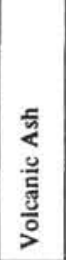 & 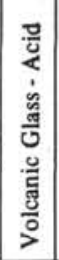 & 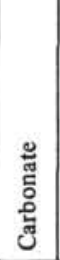 & 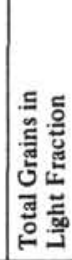 \\
\hline $51.0-1-2,8$ & & & 192 & & - & & - & 138 & 7 & 1 & 6 & - & 2 & 10 & 12 & & 12 & 1 & - & - & & 3 & 2 & & & - & & 19 & & & 387 \\
\hline $51.0-1-4$ & & 2 & 340 & 25 & & & - & 88 & - & 2 & 2 & - & - & 4 & 8 & 1 & 10 & 1 & & 2 & 1 & 6 & 2 & & - & - & & & & 7 & 498 \\
\hline $51.0-1-6,16$ & & & 235 & - & 10 & & - & 21 & 14 & - & 1 & - & - & 32 & 200 & & 6 & - & 1 & - & & 1 & 2 & & & - & & 10 & & & 513 \\
\hline $53.0-0-2$ & & - & 199 & - & & & 25 & 9 & - & - & 2 & - & - & 6 & 27 & - & 9 & 1 & & - & 2 & & - & & 1 & 6 & & & & 2 & 291 \\
\hline $53.0-6-2,66$ & & & 383 & - & - & & - & 47 & 11 & - & 2 & - & - & - & 11 & & 2 & - & - & - & & - & 1 & & & - & & 144 & & & 457 \\
\hline $53.0-7-1,150$ & & & 103 & - & - & & - & 388 & 22 & 2 & 1 & - & - & 3 & 28 & & 2 & - & - & - & & 1 & 1 & & & - & & - & & & 551 \\
\hline $53.1-1-1,7$ & & & 252 & - & - & & - & 62 & 25 & - & 3 & - & 1 & 18 & 109 & & 4 & - & - & - & & - & 2 & & & - & & 53 & & & 476 \\
\hline $53.1-1-2$ & & - & 200 & - & & & - & 15 & - & 1 & 1 & - & - & 8 & 21 & - & 2 & 1 & & - & - & - & 1 & & - & 1 & & & & 1 & 301 \\
\hline $53.1-1-4$ & & - & 311 & - & & & - & 14 & - & - & 1 & - & - & 3 & 45 & 1 & 4 & - & & 1 & - & - & 1 & & - & - & & & & - & 330 \\
\hline $53.1-1-6$ & & - & 421 & - & & & - & 34 & - & 1 & 6 & 1 & - & 9 & 91 & - & 2 & 1 & & - & - & - & 3 & & - & - & & & & 1 & 570 \\
\hline $53.1-2-3$ & & - & 130 & - & & & - & 16 & - & - & 18 & 2 & 1 & 50 & 181 & - & 4 & 2 & & - & - & - & 4 & & - & - & & & & - & 430 \\
\hline $53.1-3-3$ & & - & 153 & - & & & - & 7 & - & - & 7 & - & - & 45 & 200 & 1 & 2 & 3 & & - & - & - & 2 & & - & - & & & & - & 427 \\
\hline $53.1-3-5,23$ & & & 202 & - & 5 & - & - & 24 & & 1 & - & - & - & 44 & 175 & & 3 & - & - & - & & 1 & 2 & & & - & & 151 & & & 457 \\
\hline $53.2-1-1,21$ & & & 184 & - & 101 & - & - & 63 & & - & 2 & - & - & 19 & 170 & & 5 & 1 & - & 1 & & 1 & 4 & & & - & & 42 & & & 551 \\
\hline $53.2-1-3,22$ & & & 152 & - & 27 & - & - & 29 & & 1 & 1 & 1 & - & 42 & 177 & & 3 & - & 1 & - & & 1 & 1 & & & - & & 188 & & & 436 \\
\hline $53.2-1-5,7$ & & & 223 & - & 20 & - & - & 82 & & 1 & 4 & - & - & 29 & 192 & & 4 & - & 1 & - & & 1 & 1 & & & - & & 156 & & & 558 \\
\hline $53.1-1-4$ & & - & 311 & - & & & - & 14 & - & - & 1 & - & - & 3 & 45 & 1 & 4 & - & & 1 & - & - & 1 & & - & - & & & & - & 330 \\
\hline $54.0-1-1,74$ & - & - & 275 & - & - & - & - & 11 & - & 1 & 1 & - & - & 54 & 139 & 1 & 7 & - & - & - & 1 & - & 3 & - & - & - & - & - & - & - & 540 \\
\hline $54.0-1-3,35$ & - & - & 105 & - & - & - & - & 21 & - & - & 2 & - & - & 17 & 35 & - & 4 & - & - & - & - & 2 & - & - & - & - & - & 92 & - & - & 199 \\
\hline $54.0-2-2,23$ & - & - & 208 & - & - & - & - & 11 & - & - & 1 & - & - & 17 & $?$ & - & 4 & - & - & - & 1 & - & 2 & - & - & - & - & & - & - & $?$ \\
\hline $54.0-4-1,108$ & - & - & 309 & - & 6 & - & 1 & 33 & - & - & 1 & - & - & 24 & 64 & - & 11 & - & 2 & - & - & - & 7 & - & - & - & - & 38 & - & - & 458 \\
\hline $54.0-6-2,16$ & - & - & 256 & - & - & - & - & 25 & - & - & 2 & - & - & 47 & 238 & - & 8 & - & - & - & - & 1 & 14 & - & - & - & - & 34 & - & - & 591 \\
\hline $54.0-6-4,16$ & - & - & 142 & - & - & - & - & 11 & - & 1 & 1 & - & 1 & 47 & $?$ & - & 3 & - & - & - & - & - & 2 & - & - & - & - & - & - & - & 380 \\
\hline $54.0-6-5,17$ & - & - & 172 & - & - & - & - & 15 & - & 1 & 1 & - & - & 39 & 123 & 2 & 9 & - & - & 1 & - & - & - & - & - & 1 & - & - & - & - & 378 \\
\hline $54.0-7-2,34$ & - & - & 181 & - & 14 & - & - & 89 & - & - & 1 & - & - & 12 & - & - & 1 & - & - & - & - & - & 1 & - & 25 & - & - & 37 & - & - & 324 \\
\hline $55.0-3-3,14$ & - & - & - & - & - & - & - & - & - & - & - & - & - & - & 6 & - & - & - & - & - & - & - & - & - & - & - & - & 44 & - & - & - \\
\hline $55.0-3-5,15$ & - & - & - & - & - & - & - & - & - & - & - & - & - & - & 1 & - & - & - & - & - & - & - & - & - & - & - & - & 11 & - & - & - \\
\hline $55.0-4-4,145$ & - & - & - & - & - & - & - & - & - & - & - & - & - & - & 15 & - & - & - & - & - & - & - & - & - & - & - & - & - & - & - & - \\
\hline $55.0-4-6,145$ & - & - & 60 & - & - & - & - & 108 & - & - & - & - & - & - & 2 & - & 1 & - & - & - & - & - & - & - & - & - & - & - & - & - & - \\
\hline
\end{tabular}




\begin{tabular}{|c|c|c|c|c|c|c|c|c|c|c|c|c|c|c|c|c|c|c|c|c|c|c|c|c|c|c|c|c|c|c|c|}
\hline $\begin{array}{c}\text { Sample } \\
\text { Designation }\end{array}$ & 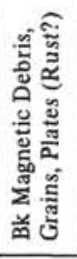 & 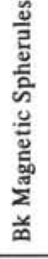 & 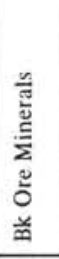 & 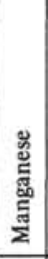 & 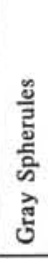 & $\begin{array}{l}\overrightarrow{\mathrm{z}} \\
\vec{\alpha}\end{array}$ & 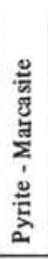 & 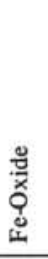 & 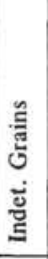 & 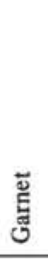 & 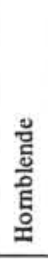 & 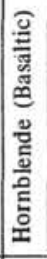 & 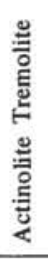 & 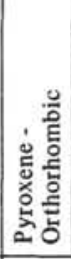 & 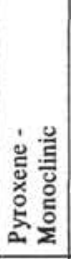 & 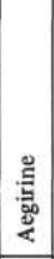 & 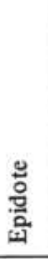 & 产 & 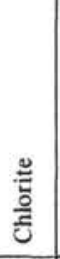 & 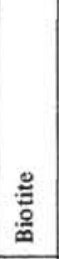 & 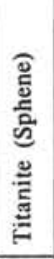 & $\begin{array}{l}\text { ¿ } \\
\text { స్ } \\
\end{array}$ & 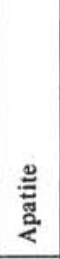 & 胥 & 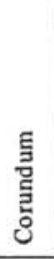 & 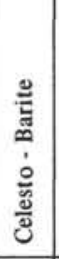 & 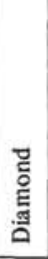 & 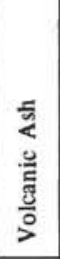 & 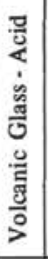 & 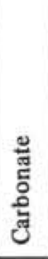 & 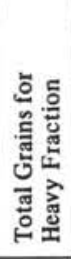 \\
\hline $55.0-6-1,7$ & - & - & - & - & - & - & - & - & - & - & - & - & - & - & - & - & - & - & - & - & - & - & - & - & - & - & - & 42 & - & - & - \\
\hline $55.0-6-5,16$ & - & - & - & - & - & - & - & - & - & - & - & - & - & - & 4 & - & - & - & - & - & - & - & - & - & - & - & - & 29 & - & - & - \\
\hline $55.0-7-3,18$ & - & - & - & - & - & - & - & - & - & - & - & - & - & - & - & - & - & - & - & - & - & - & - & - & - & - & - & 78 & - & - & - \\
\hline $57.1-2-1,44$ & - & - & 67 & - & - & - & 2 & 8 & - & - & 1 & - & - & 13 & 43 & - & - & - & - & - & - & - & 2 & - & - & 1 & - & 36 & - & - & 556 \\
\hline $57.1-3-2,109$ & - & - & 45 & - & - & - & - & 31 & - & - & - & - & 1 & 7 & 57 & - & 1 & 1 & - & - & - & - & 3 & - & - & - & - & - & - & - & 336 \\
\hline after $\mathrm{HCl}$ & - & - & 45 & - & - & - & - & 31 & - & - & - & - & 1 & 7 & 57 & - & 1 & 1 & - & - & - & - & 3 & - & - & - & - & - & - & - & 336 \\
\hline $57.1-4-2,20$ & - & - & 28 & - & - & - & - & 6 & - & - & 1 & - & - & 4 & 35 & - & 2 & - & - & - & - & - & - & - & - & - & - & 166 & - & - & 522 \\
\hline $57.1-4-5,145$ & - & - & 3 & - & - & - & - & - & - & - & - & - & - & 1 & 25 & - & - & 1 & - & - & - & - & 48 & - & - & - & - & - & - & - & 479 \\
\hline after $\mathrm{HCl}$ & - & - & 3 & - & - & - & - & - & - & - & - & - & - & 1 & 25 & - & - & 1 & - & - & - & - & 48 & - & - & - & - & - & - & - & 479 \\
\hline
\end{tabular}


TABLE 4B

Mineralogical Analysis of Light Fraction in Size Range $0.05-0.01 \mathrm{~mm}$

\begin{tabular}{|c|c|c|c|c|c|c|c|c|c|c|c|c|c|c|c|c|c|c|c|c|c|c|c|c|c|c|c|c|c|c|c|c|}
\hline $\begin{array}{c}\text { Sample } \\
\text { Designation }\end{array}$ & 胥 & 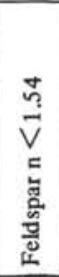 & 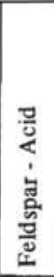 & 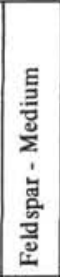 & 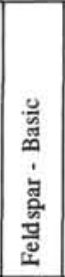 & 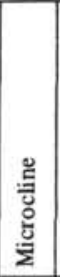 & 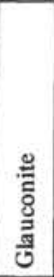 & 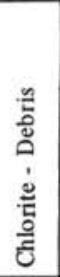 & 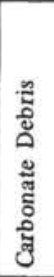 & 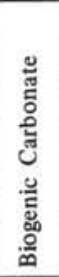 & 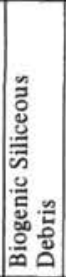 & 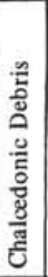 & 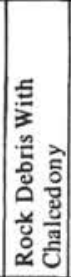 & 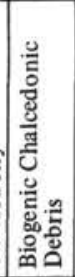 & 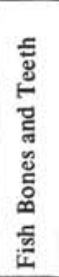 & 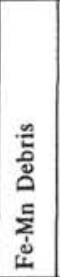 & 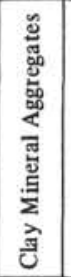 & 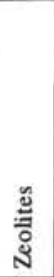 & 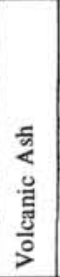 & 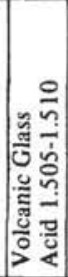 & 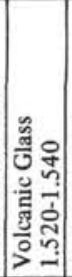 & 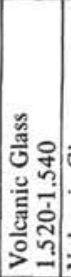 & 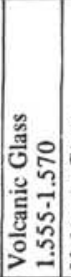 & 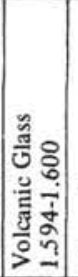 & 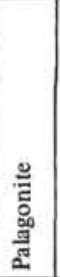 & 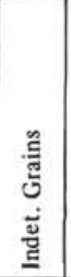 & 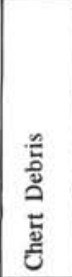 & 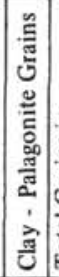 & 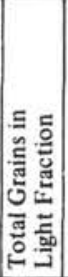 & & 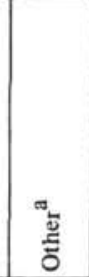 & \\
\hline $51.0-1-2,8$ & 13 & 1 & 1 & & 7 & & & 1 & & - & 6 & & & & 28 & & 275 & 37 & & & 93 & - & & 2 & & 1 & 32 & & & 516 & & \\
\hline $51.0-1-4$ & 43 & 3 & 2 & 9 & 9 & & & - & - & 1 & 1 & - & 12 & - & 37 & 30 & & 3 & & & 65 & & - & & & - & 13 & & 28 & 233 & $\begin{array}{r}4 \mathrm{~V} \\
13 \mathrm{R}\end{array}$ & ${ }_{1} \mathrm{P}$ \\
\hline $51.0-1-6,16$ & 2 & 11 & - & & 2 & & & 2 & & - & 3 & & & & 313 & & 33 & 43 & & & 4 & - & & - & & 5 & 57 & & & 494 & & \\
\hline $53.0-0-2$ & 6 & - & 3 & 20 & 20 & & & - & - & - & 2 & - & - & 1 & 1 & - & & 1 & & & 250 & & 3 & & & - & 111 & & - & 421 & $\begin{array}{r}3 \mathrm{~V} \\
26 \mathrm{R}\end{array}$ & ${ }_{1} \mathrm{P}$ \\
\hline $53.0-6-2,66$ & 10 & 213 & 2 & & 2 & & & 3 & & 106 & - & & & & - & & 74 & - & & & 14 & - & & - & & - & - & & & 568 & & \\
\hline $53.0-7-1,150$ & 1 & 7 & 8 & & 160 & & & - & & - & - & & & & 3 & & 9 & - & & & - & - & & - & & - & 223 & & & 411 & & \\
\hline $53.1-1-1,7$ & 16 & - & 2 & & 211 & & & 2 & & - & 3 & & & & 11 & & 75 & - & & & 2 & - & & 2 & & - & 22 & & & 399 & & \\
\hline $53.1-1-2$ & 19 & 5 & 11 & 148 & 148 & & & - & - & 1 & 1 & 2 & - & - & 12 & - & & 1 & & & 4 & & - & & & - & 46 & & 1 & 272 & $\begin{array}{r}2 \mathrm{~V} \\
15 \mathrm{R}\end{array}$ & ${ }_{1}^{\mathrm{P}}$ \\
\hline $53.1-1-4$ & 13 & 2 & 13 & 181 & 181 & & & - & - & 1 & - & - & - & 6 & 21 & - & & 1 & & & 5 & & - & & & - & & & 8 & 253 & $\begin{array}{r}6 \mathrm{~V} \\
13 \mathrm{R}\end{array}$ & ${ }_{1}^{\mathrm{P}}$ \\
\hline $53.1-1 \cdot 6$ & 8 & - & 25 & 209 & 209 & & & - & - & - & - & - & - & 3 & 2 & - & & 1 & & & 2 & & - & & & - & - & & 82 & 369 & $\begin{array}{r}2 \mathrm{~V} \\
34 \mathrm{R}\end{array}$ & ${ }_{1}^{P}$ \\
\hline $53.1-2-3$ & 2 & - & 4 & 46 & 46 & & & - & - & - & 1 & - & - & 1 & - & - & & 1 & & & 81 & & 17 & & & - & 4 & & - & 326 & $\begin{array}{r}25 \mathrm{~V} \\
163^{\mathrm{R}}\end{array}$ & ${ }_{2} \mathrm{P}$ \\
\hline $53.1-2-4$ & 2 & - & 5 & 34 & 34 & & & - & - & - & 3 & - & - & 1 & 1 & - & & 1 & & & 123 & & 16 & & & - & 4 & & - & 368 & $\begin{array}{r}27 \mathrm{~V} \\
168 \mathrm{R}\end{array}$ & ${ }_{1} \mathrm{P}$ \\
\hline $53.1-2-6$ & 3 & - & 4 & 28 & 28 & & & - & - & - & 3 & - & - & - & - & - & & 1 & & & 70 & & 92 & & & - & - & & 7 & 377 & $\begin{array}{r}69 \mathrm{~V} \\
185^{\mathrm{R}}\end{array}$ & ${ }_{1} \mathrm{P}$ \\
\hline $53.1-3-3$ & 1 & - & 2 & 34 & 34 & & & - & - & 1 & 1 & - & - & 1 & 1 & - & & - & & & 120 & & 9 & & & - & 100 & & - & 301 & $\begin{array}{l}30 \mathrm{~V} \\
32 \mathrm{R}\end{array}$ & ${ }_{1} \mathrm{P}$ \\
\hline $53.1-3-5,23$ & 1 & 1 & - & & 29 & & & - & & - & 2 & & & & - & & - & - & & & - & & 137 & 25 & & - & - & & & 346 & & \\
\hline $53.2-1-1,21$ & 7 & 4 & - & & 301 & & & - & & - & - & & & & 7 & & 77 & - & & & 5 & & 2 & 2 & & 1 & - & & & 448 & & \\
\hline $53.2-1-3,22$ & - & - & - & & 49 & & & - & & - & 4 & & & & - & & 23 & - & & & - & & 39 & 23 & & 1 & - & & & 327 & & \\
\hline $53.2-1-5,7$ & 3 & 2 & - & & 52 & & & - & & - & 12 & & & & - & & 14 & - & & & - & & 233 & 17 & & 2 & - & & & 491 & & \\
\hline $54.0-1-1,74$ & 1 & - & 3 & 33 & 33 & - & - & - & - & - & 3 & - & - & 3 & - & - & - & 1 & - & - & 155 & - & 26 & - & - & - & 59 & - & - & 454 & $\begin{array}{l}19 \mathrm{~V} \\
23 \mathrm{R}\end{array}$ & ${ }_{1} \mathrm{P}$ \\
\hline $54,0-1-3,35$ & 7 & - & - & - & 20 & - & - & - & - & - & 3 & - & - & - & - & - & - & - & - & - & - & 238 & 9 & 2 & - & - & - & - & - & 371 & $\begin{array}{l}20 \mathrm{~V} \\
15 \mathrm{R}\end{array}$ & ${ }_{1}^{\mathrm{P}}$ \\
\hline $54.0-2-2,23$ & - & - & 2 & 20 & 20 & - & - & - & - & - & 3 & - & - & - & 1 & - & - & 1 & - & - & 253 & - & 8 & - & - & - & 1 & - & - & 275 & & \\
\hline $54.0-4-1,108$ & - & - & 21 & - & - & - & - & 2 & - & - & 5 & - & - & - & - & - & - & - & - & - & - & 262 & 13 & 3 & - & 2 & 3 & - & - & 349 & & \\
\hline $54.0-6-2,16$ & $?$ & - & - & - & 31 & - & - & - & - & - & - & - & - & - & - & - & - & - & - & - & - & 405 & 8 & 7 & - & - & - & - & - & 486 & & \\
\hline $54.0-6-4,16$ & - & - & 4 & 47 & 47 & - & - & - & - & - & 2 & - & - & - & 3 & - & - & 1 & - & - & 134 & - & 18 & - & - & - & ? & - & - & 275 & ${ }_{8} \mathrm{~V}$ & ${ }_{1} \mathrm{P}$ \\
\hline
\end{tabular}




\begin{tabular}{|c|c|c|c|c|c|c|c|c|c|c|c|c|c|c|c|c|c|c|c|c|c|c|c|c|c|c|c|c|c|c|c|c|}
\hline $\begin{array}{c}\text { Sample } \\
\text { Designation }\end{array}$ & 营 & 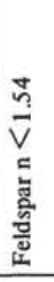 & 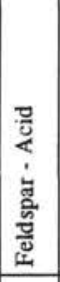 & 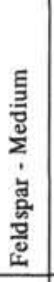 & 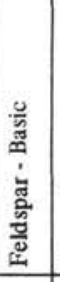 & 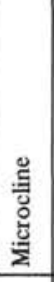 & 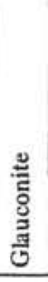 & 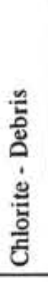 & 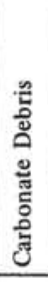 & 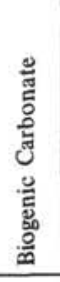 & 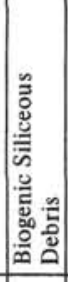 & 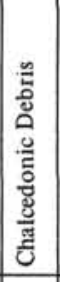 & 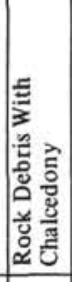 & 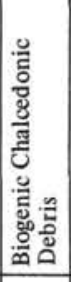 & 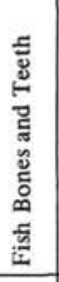 & 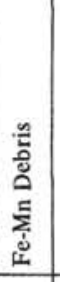 & 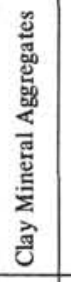 & 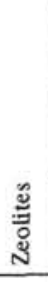 & 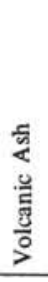 & 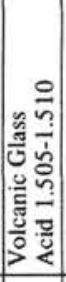 & 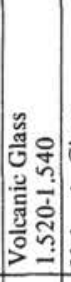 & 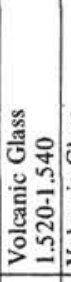 & 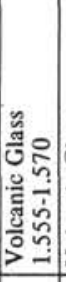 & 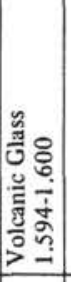 & 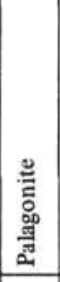 & 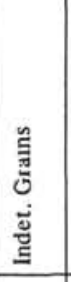 & 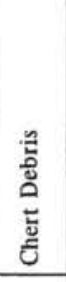 & 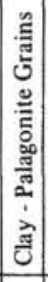 & 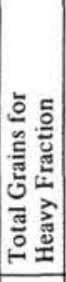 & & 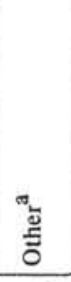 & \\
\hline $54.0-6-5,17$ & - & - & - & 17 & 17 & - & - & - & - & - & 2 & - & - & - & - & - & - & 1 & - & - & 246 & - & 11 & - & - & - & 66 & - & - & 361 & $17 \mathrm{~V}$ & ${ }_{1} \mathrm{P}$ \\
\hline $54.0-7-2,34$ & 10 & - & - & - & 10 & - & - & - & - & - & 9 & - & - & - & - & - & - & - & - & - & - & 275 & 5 & 6 & - & 1 & 1 & - & - & 354 & & \\
\hline $55.0-3-3,14$ & - & - & - & - & 15 & - & - & - & - & 7 & 15 & - & - & - & - & - & - & - & - & - & - & 18 & 5 & 1 & - & - & 4 & - & - & 124 & & \\
\hline $55.0-3-5,15$ & 4 & 2 & 1 & 1 & 6 & - & - & - & - & 257 & 12 & - & - & - & - & - & 13 & - & - & - & - & 15 & - & 1 & - & 1 & 348 & - & - & 7 & & \\
\hline $55.0-4-4,145$ & 8 & 4 & 2 & 12 & 38 & - & - & - & - & 28 & 23 & - & - & - & - & - & - & - & - & - & - & 18 & - & - & - & 2 & 190 & - & - & 20 & & \\
\hline $55.0-4-6,145$ & 13 & 1 & 5 & - & 5 & - & - & 11 & - & 5 & 26 & - & - & - & - & - & - & - & - & - & - & 10 & - & - & - & 5 & 278 & - & - & 26 & & \\
\hline $55.0-5-3,1$ & 11 & 3 & 2 & - & 48 & - & - & 51 & - & 4 & 25 & - & - & - & - & - & 14 & - & - & - & - & 3 & - & - & - & 28 & 290 & - & - & 22 & & \\
\hline $55.0-6-1,7$ & 5 & - & 1 & - & 11 & - & - & 8 & - & 6 & 137 & - & - & - & 1 & - & 18 & - & - & - & - & 11 & - & - & - & 15 & 267 & - & - & 12 & & \\
\hline $55.0-6-5,16$ & 1 & 1 & 1 & - & 6 & - & - & 7 & - & - & 177 & - & - & - & - & - & 27 & - & - & - & - & 11 & - & 1 & - & 1 & 284 & - & - & - & & \\
\hline $55.0-7-3,18$ & 8 & 2 & - & - & 9 & - & - & 3 & - & - & 116 & - & - & - & - & - & 20 & - & - & - & - & 12 & - & 2 & - & 3 & 253 & - & - & - & & \\
\hline $57.1-2-1,44$ & - & - & - & - & 4 & - & - & - & - & - & 138 & - & - & - & - & - & 5 & - & - & - & - & 5 & - & 187 & - & - & - & - & - & 375 & & \\
\hline $57.1-3-2,109$ & - & - & - & 3 & 3 & - & - & 1 & $?$ & 32 & 15 & - & - & 1 & 1 & -1 & - & 1 & - & - & 6 & - & 2 & - & - & 2 & - & - & 9 & 289 & & \\
\hline after $\mathrm{HCl}$ & - & $?$ & - & 3 & 3 & - & - & - & - & - & 11 & - & - & - & - & - & - & 1 & - & - & 5 & - & 2 & - & - & 9 & 42 & - & - & 221 & & \\
\hline $57.1-4-2,20$ & 1 & - & - & - & 10 & - & - & - & - & - & 41 & - & - & - & - & - & 20 & - & - & - & - & 22 & - & 169 & - & - & - & - & - & 430 & & \\
\hline $57.1-4-5,145$ & - & - & - & - & - & - & - & - & - & - & 40 & - & - & - & 5 & - & - & - & - & - & 15 & - & - & - & - & 5 & - & - & 7 & 317 & & ${ }_{1} \mathrm{P}$ \\
\hline after $\mathrm{HCl}$ & - & - & 1 & 4 & 4 & - & - & - & - & - & 3 & - & - & 2 & - & - & - & - & - & - & 11 & - & 11 & - & - & 8 & - & - & - & & & $1^{\mathrm{P}}$ \\
\hline
\end{tabular}


TABLE 5

Quartz and Carbonate Minerals as Determined by X-ray Analysis

\begin{tabular}{|c|c|c|c|c|c|c|}
\hline Sample No. & Fraction (mm) & Quartz \% & Calcite $\%$ & Aragonite \% & Feldspar & Cristobalite \\
\hline $45.1-1-1,7$ & $0.05-0.01$ & 3.0 & - & - & + & - \\
\hline $45.1-1-2,100$ & $0.05-0.01$ & 1.0 & - & - & + & - \\
\hline $46.0-1 \cdot 1,145$ & $0.05-0.01$ & 45.0 & - & - & + & - \\
\hline $46.0-1-2,145$ & $0.05-0.01$ & 50.0 & - & - & + & - \\
\hline $46.0-1-3,145$ & $0.05-0.01$ & 30.0 & - & - & + & - \\
\hline $46.0-1-4,145$ & $0.05-0.01$ & $75.0(?)$ & - & - & + & - \\
\hline $46.0-1-5,145$ & $0.05-0.01$ & 50.0 & - & - & + & - \\
\hline $47.0-1-1,17$ & $0.05-0.01$ & 30.0 & 22.0 & - & + & - \\
\hline $47.2-1-2,23$ & $0.05-0.01$ & 27.0 & 30.0 & - & + & - \\
\hline $47.2-1-2,181$ & $0.05-0.01$ & 30.0 & 60.0 & - & + & - \\
\hline $47.2-1-3,90$ & $0.05-0.01$ & 25.0 & 70.0 & - & + & - \\
\hline $47.2-2-2,8$ & $0.05-0.01$ & 22.0 & 70.0 & - & + & - \\
\hline $47.2-2-6,6$ & $0.05-0.01$ & 10.0 & 85.0 & - & + & - \\
\hline $47.2-3-3,6$ & $0.05-0.01$ & 72.0 & 52.0 & - & + & - \\
\hline $47.2-4-1,16$ & $0.05-0.01$ & 8.0 & 90.0 & $<1.0$ & - & - \\
\hline $47.2-4-3,16$ & $0.05-0.01$ & 5.0 & 94.0 & - & - & - \\
\hline $47.2-4-5,16.5$ & $0.05-0.01$ & 10.0 & 62.0 & - & + & - \\
\hline $47.2-5-1,17$ & $0.05-0.01$ & 7.0 & 65.0 & - & + & - \\
\hline $47.2-5-3,6$ & $0.05-0.01$ & 18.0 & 80.0 & - & + & - \\
\hline $47.2-5-5,6$ & $0.05-0.01$ & 10.0 & 78.0 & - & + & - \\
\hline $47.2-7-1,17$ & $0.05-0.01$ & 20.0 & 75.0 & - & + & - \\
\hline $47.2-7-3,13$ & $0.05-0.01$ & - & 100.0 & - & - & - \\
\hline $47.2-7-5,27.5$ & $0.05-0.01$ & - & 100.0 & - & - & - \\
\hline $47.2-8-1,27$ & $0.05-0.01$ & - & 100.0 & - & - & - \\
\hline $47.2-8 \cdot 3,18$ & $0.05-0.01$ & - & 100.0 & - & - & - \\
\hline $47.2-8-5,17$ & $0.05-0.01$ & - & 100.0 & - & - & - \\
\hline $47.2-9-1,16$ & $0.05-0.01$ & - & 100.0 & - & - & - \\
\hline $47.2-9 \cdot 3,17$ & $0.05-0.01$ & - & 100.0 & - & - & - \\
\hline $47.2-10-2,17$ & $0.05-0.01$ & 2.0 & 97.0 & 1.0 & - & - \\
\hline $47.2-10-6,16$ & $0.05-0.01$ & - & 100.0 & - & - & - \\
\hline $47.2-11-2,145$ & $0.05-0.01$ & - & 100.0 & - & - & - \\
\hline $47.2-12-2,17$ & $0.05-0.01$ & 3.0 & 97.0 & - & - & - \\
\hline $47.2-12-4,145$ & $0.05-0.01$ & 1.0 & 85.0 & - & - & - \\
\hline $47.2-13-2,145$ & $0.05-0.01$ & 2.0 & 97.0 & - & - & - \\
\hline $47.2-14-4,145$ & $0.05-0.01$ & - & 100.0 & - & - & - \\
\hline $47.2-14-6,145$ & $0.05-0.01$ & - & 100.0 & - & - & - \\
\hline $48.2-1-2,33$ & $0.05-0.01$ & 7.0 & 92.0 & - & - & - \\
\hline $48.2-1-4,16$ & $0.05-0.01$ & 8.0 & 60.0 & - & - & - \\
\hline $48.2-1-6,11$ & $0.05-0.01$ & 1.0 & 98.0 & - & - & - \\
\hline
\end{tabular}


TABLE 5 - Continued

\begin{tabular}{|c|c|c|c|c|c|c|}
\hline Sample No. & Fraction $(\mathrm{mm})$ & Quartz \% & Calcite $\%$ & Aragonite $\%$ & Feldspar & Cristobalite \\
\hline $48.2-2-5,16$ & $0.05-0.01$ & - & 100.0 & - & - & - \\
\hline $48.2 \cdot 3-1,117$ & $0.05-0.01$ & - & 100.0 & - & - & - \\
\hline $48.2-3-3,17$ & $0.05-0.01$ & - & 100.0 & - & - & - \\
\hline $48.2-3-5,16$ & $0.05-0.01$ & - & 100.0 & - & - & - \\
\hline $49.0-1-1,11$ & $0.05-0.01$ & 19.0 & - & - & + & - \\
\hline $49.0-1-2,16$ & $0.05-0.01$ & 16.0 & 5.0 & - & + & - \\
\hline $49.0-1-4,6$ & $0.05-0.01$ & 20.0 & - & - & + & - \\
\hline $49.0-1-5,70$ & $0.05-0.01$ & 22.0 & - & - & + & - \\
\hline $49.0-2-1,70$ & $0.05-0.01$ & 20.0 & 32.0 & - & + & - \\
\hline $49.1-1-1,77$ & $0.05-0.01$ & 15.0 & 1.0 & - & + & - \\
\hline $49.1-1-4,17$ & $0.05-0.01$ & 7.0 & $<1.0$ & - & + & - \\
\hline $49.1-2-2,145$ & $0.05-0.01$ & 45.0 & 37.0 & - & + & - \\
\hline $50.1-1-4,17$ & $0.05-0.01$ & 27.0 & 8.0 & - & + & - \\
\hline $50.1-2-3,4$ & $0.05-0.01$ & 15.0 & - & - & + & - \\
\hline $50.1-3-2,73$ & $0.05-0.01$ & 27.0 & - & - & + & - \\
\hline $45.1-1-1,7$ & $<0.01$ & 12.0 & $<1.0$ & - & + & - \\
\hline $45.1-1-2,100$ & $<0.01$ & 5.0 & - & - & + & - \\
\hline $46.0-1-2,145$ & $<0.01$ & 25.0 & - & - & + & - \\
\hline $46.0-1-5,145$ & $<0.01$ & 27.0 & 2.0 & - & + & - \\
\hline $46.0-1-6,145$ & $<0.01$ & 37.0 & - & - & + & - \\
\hline $50.0-4-1,75$ & $<0.01$ & 22.0 & - & - & + & - \\
\hline $50.1-3-4,16$ & $<0.01$ & 28.0 & - & - & + & - \\
\hline $51.0-1-2,8$ & $<0.01$ & 25.0 & 2.0 & - & + & - \\
\hline $51.0-1-4,6$ & $<0.01$ & 35.0 & $<1.0$ & - & + & - \\
\hline $51.0-1-6,17$ & $<0.01$ & 20.0 & - & - & + & - \\
\hline $51.1-1 \cdot 2,16$ & $<0.01$ & 22.0 & - & - & + & - \\
\hline $51.1-1-4,16$ & $<0.01$ & 20.0 & - & - & + & - \\
\hline $51.1-1-6,16$ & $<0.01$ & 20.0 & trace & - & - & - \\
\hline $51.1-2-2,8$ & $<0.01$ & 25.0 & - & - & + & - \\
\hline $52.0-1-4,8$ & $<0.01$ & 37.0 & - & - & + & - \\
\hline $52.0-2-3,9$ & $<0.01$ & 20.0 & - & - & + & - \\
\hline $52.0-3-3,16$ & $<0.01$ & 20.0 & - & - & + & - \\
\hline $52.0-4-2,7$ & $<0.01$ & 17.0 & - & - & + & - \\
\hline $52.0-5-2,17$ & $<0.01$ & 18.0 & $<1.0$ & - & - & - \\
\hline $52.0-8-4,145$ & $<0.01$ & 30.0 & - & - & + & + \\
\hline $52.0-8-5,145$ & $<0.01$ & 30.0 & - & - & + & - \\
\hline $52.0-8-6,145$ & $<0.01$ & 32.0 & 1.0 & - & + & - \\
\hline $53.0-1-3,17$ & $<0.01$ & 5.0 & - & - & + & - \\
\hline $53.1-1-4,17$ & $<0.01$ & 12.0 & 1.0 & - & + & - \\
\hline
\end{tabular}


TABLE 5 - Continued

\begin{tabular}{|c|c|c|c|c|c|c|}
\hline Sample No. & Fraction $(\mathrm{mm})$ & Quartz \% & Calcite \% & Aragonite $\%$ & Feldspar & Cristobalite \\
\hline $53.1-2-4,10$ & $<0.01$ & 3.0 & 1.0 & - & + & - \\
\hline $53.1-3-5,23$ & $<0.01$ & 2.0 & 1.0 & - & + & - \\
\hline $54.0-1-1,74$ & $<0.01$ & 2.0 & - & - & + & - \\
\hline $54.0-1-3,35$ & $<0.01$ & 1.0 & - & - & + & - \\
\hline $54.0-6-2,16$ & $<0.01$ & 1.0 & - & - & - & - \\
\hline $54.0-6-5,17$ & $<0.01$ & 1.0 & - & - & - & - \\
\hline $57.1-2-1,44$ & $<0.01$ & - & 62.0 & - & - & - \\
\hline $57.1-3-2,109$ & $<0.01$ & - & 98.0 & - & - & - \\
\hline $57.1-4-2,20$ & $<0.01$ & - & 100.0 & - & - & - \\
\hline $57.1-4-5,145$ & $<0.01$ & $<1.0$ & 52.0 & - & - & - \\
\hline $44.0-2-6,145$ & 0.001 & - & 100.0 & - & - & - \\
\hline $45.1-1-2,100$ & 0.001 & 2.0 & - & - & + & - \\
\hline $46.0-1-2,145$ & 0.001 & 13.0 & - & - & + & - \\
\hline $46.0-1-3,145$ & 0.001 & 18.0 & - & - & + & - \\
\hline $46.0-1-4,145$ & 0.001 & 12.0 & - & - & + & - \\
\hline $46.0-1-6,145$ & 0.001 & 25.0 & - & - & + & - \\
\hline $47.0-1-1,17$ & 0.001 & 18.0 & 72.0 & - & + & - \\
\hline $47.0-1-4,90$ & 0.001 & 10.0 & - & - & + & - \\
\hline $47.0-1-6,14$ & 0.001 & - & 100.0 & - & - & - \\
\hline $47.1-1-2,16$ & 0.001 & - & 100.0 & - & - & - \\
\hline $47.2-1-2,23$ & 0.001 & 3.0 & 95.0 & - & - & - \\
\hline $47.2-1-3,90$ & 0.001 & 2.0 & 97.0 & - & - & - \\
\hline $47.2-2-6,6$ & 0.001 & 2.0 & 97.0 & - & - & - \\
\hline $47.2-2-6,7$ & 0.001 & 7.0 & 90.0 & - & - & - \\
\hline 47.2-3-CC & 0.001 & 1.0 & 98.0 & - & - & - \\
\hline $47.2-4-3,16$ & 0.001 & - & 100.0 & - & - & - \\
\hline $47.2-4-5,16.5$ & 0.001 & 1.0 & 97.0 & - & - & - \\
\hline $47.2-5-1,17$ & 0.001 & 1.0 & 96.0 & - & - & - \\
\hline $47.2-5-3,6$ & 0.001 & 1.0 & 98.0 & - & - & - \\
\hline $47.2-5-5,6$ & 0.001 & 1.0 & 98.0 & - & - & - \\
\hline $47.2-6-2,6$ & 0.001 & 1.0 & 97.0 & - & - & - \\
\hline $47.2-7-1,17$ & 0.001 & 2.0 & 97.0 & - & - & - \\
\hline $47.2-7-3,13$ & 0.001 & 2.0 & 65.0 & - & - & - \\
\hline $47.2-7-5,27.5$ & 0.001 & 3.0 & 60.0 & - & - & - \\
\hline $47.2-8-1,27$ & $<0.001$ & - & 100.0 & - & - & - \\
\hline $47.2-8-3,18$ & $<0.001$ & - & 98.0 & - & - & - \\
\hline $47.2-8-5,17$ & $<0.001$ & $<1.0$ & 98.0 & $<1.0$ & - & - \\
\hline $47.2-9-1,16$ & $<0.001$ & - & 100.0 & - & - & - \\
\hline $47.2-9-3,17$ & $<0.001$ & - & 98.0 & - & - & - \\
\hline
\end{tabular}


TABLE 5 - Continued

\begin{tabular}{|c|c|c|c|c|c|c|}
\hline Sample No. & Fraction (mm) & Quartz \% & Calcite \% & Aragonite $\%$ & Feldspar & Cristobalite \\
\hline $47.2-10$, top & $<0.001$ & - & 100.0 & - & - & - \\
\hline $47.2-10-2,17$ & $<0.001$ & - & 98.0 & - & - & - \\
\hline $47.2-10-4,17$ & $<0.001$ & - & 100.0 & - & - & - \\
\hline $47.2-10-6,16$ & $<0.001$ & - & 100.0 & - & - & - \\
\hline $47.2-11-2,145$ & $<0.001$ & - & 100.0 & - & - & - \\
\hline $47.2-12-4,145$ & $<0.001$ & - & 100.0 & - & - & - \\
\hline $47.2-13-6,145$ & $<0.001$ & - & 100.0 & - & - & - \\
\hline $47.2-14-3,145$ & $<0.001$ & - & 98.0 & - & - & - \\
\hline $47.2-14-4,145$ & $<0.001$ & - & 98.0 & - & - & - \\
\hline $47.2-14-6,145$ & $<0.001$ & - & 100.0 & - & - & - \\
\hline $48.2 \cdot 1-2,33$ & $<0.001$ & - & 98.0 & - & - & - \\
\hline $48.2-1-4,16$ & $<0.001$ & - & 98.0 & - & - & - \\
\hline $48.2-1-6,11$ & $<0.001$ & - & 98.0 & - & - & - \\
\hline $48.2-2-3,145$ & $<0.001$ & - & 100.0 & - & - & - \\
\hline $48.2-2-5,16$ & $<0.001$ & - & 100.0 & - & - & - \\
\hline $48.2-3-1,117$ & $<0.001$ & - & 100.0 & - & - & - \\
\hline $48.2-3-3,17$ & $<0.001$ & - & 100.0 & - & - & - \\
\hline $48.2-3-5,16$ & $<0.001$ & - & 98.0 & - & - & - \\
\hline $49.0-1-1,11$ & $<0.001$ & 10.0 & 1.0 & - & + & - \\
\hline $49.0-1-2,16$ & $<0.001$ & 15.0 & $<1.0$ & - & + & - \\
\hline $49.0-1-2,124$ & $<0.001$ & 15.0 & $<1.0$ & - & + & - \\
\hline $49.0-1-4,6$ & $<0.001$ & 10.0 & - & - & + & - \\
\hline $49.0-1-5,70$ & $<0.001$ & 18.0 & - & - & + & - \\
\hline $49.0-2-1,70$ & $<0.001$ & - & 98.0 & - & - & - \\
\hline $49.1-1-1,77$ & $<0.001$ & 7.0 & $<1.0$ & - & - & - \\
\hline $49.1-1-2,124$ & $<0.001$ & 15.0 & 1.0 & - & - & - \\
\hline $49.1-1-5,38$ & $<0.001$ & $<1.0$ & 98.0 & - & - & - \\
\hline $50.1-1-2,15$ & $<0.001$ & 10.0 & - & - & + & - \\
\hline $50.1-1 \cdot 4,17$ & $<0.001$ & 21.0 & - & - & + & - \\
\hline $50.1-1-6,16$ & $<0.001$ & 22.0 & - & - & + & - \\
\hline $50.1-4-1,75$ & $<0.001$ & 10.0 & - & - & + & - \\
\hline $51.0-1-2,8$ & $<0.001$ & 13.0 & - & - & + & - \\
\hline $51.0-1-6,16$ & $<0.001$ & 8.0 & - & - & + & - \\
\hline $51.1-1-4,16$ & $<0.001$ & $<1.0$ & - & - & + & - \\
\hline $51.1-1-6,16$ & $<0.001$ & 22.0 & - & - & + & - \\
\hline $51.1-2-2,8$ & $<0.001$ & 18.0 & - & - & + & - \\
\hline $52.0-1-2,8$ & $<0.001$ & 18.0 & - & - & + & - \\
\hline $52.0-1 \cdot 4,8$ & $<0.001$ & 13.0 & - & - & + & - \\
\hline $52.0-1 \cdot 6,9$ & $<0.001$ & 15.0 & 1.0 & - & + & - \\
\hline
\end{tabular}


TABLE 5 - Continued

\begin{tabular}{|c|c|c|c|c|c|c|}
\hline Sample No. & Fraction (mm) & Quartz \% & Calcite \% & Aragonite $\%$ & Feldspar & Cristobalite \\
\hline $52 \cdot 0-2 \cdot 3,7$ & $<0.001$ & 13.0 & 1.0 & - & + & - \\
\hline $52.0-2-5,18$ & $<0.001$ & 12.0 & - & - & + & - \\
\hline $52.0-3-1,16$ & $<0.001$ & 15.0 & - & - & + & - \\
\hline $52.0-3-3,16$ & $<0.001$ & 3.0 & - & - & + & - \\
\hline $52.0-3-5,7$ & $<0.001$ & 20.0 & 1.0 & - & + & - \\
\hline $52.0-4-2,7$ & $<0.001$ & 10.0 & - & - & + & - \\
\hline $52.0-4-4,6$ & $<0.001$ & 7.0 & - & - & + & - \\
\hline $52.0-4-6,18$ & $<0.001$ & 18.0 & $<1.0$ & - & + & - \\
\hline $52.0-5-1,127$ & $<0.001$ & 20.0 & $<1.0$ & - & + & - \\
\hline $52.0-5-2,17$ & $<0.001$ & 25.0 & $<1.0$ & - & + & - \\
\hline $52.0-5-3,18$ & $<0.001$ & 20.0 & - & - & + & - \\
\hline $52.0-5-5,18$ & $<0.001$ & 20.0 & - & - & + & - \\
\hline $52.0-7-2,37$ & $<0.001$ & 30.0 & 1.0 & - & + & - \\
\hline $52.0-8-2,145$ & $<0.001$ & 20.0 & - & - & + & - \\
\hline $52.0-8-5,145$ & $<0.001$ & 20.0 & 12.0 & - & + & + \\
\hline $52.0-8-6,145$ & $<0.001$ & 30.0 & - & - & + & - \\
\hline $53.0-1-1,10$ & $<0.001$ & $<1.0$ & - & - & + & - \\
\hline $53.0-2-\mathrm{CC}$ & $<0.001$ & $<1.0$ & - & - & - & - \\
\hline $53.0-6-2,66$ & $<0.001$ & $<1.0$ & 6.0 & - & - & - \\
\hline $53.0-7-1,150$ & $<0.001$ & 1.0 & - & - & + & - \\
\hline $53.1-1-2,70$ & $<0.001$ & 6.0 & - & - & + & - \\
\hline $53.1-1-6,28$ & $<0.001$ & 6.0 & - & $<1.0$ & + & - \\
\hline $53.1-2-4,10$ & $<0.001$ & 3.0 & $<1.0$ & - & + & - \\
\hline $53.1-2-6,7$ & $<0.001$ & 2.0 & - & - & + & - \\
\hline $53.1-3-2,40$ & $<0.001$ & 1.0 & - & - & + & - \\
\hline $53.1-3-5,22$ & $<0.001$ & - & $<1.0$ & - & - & - \\
\hline $53.2-1-1,21$ & $<0.001$ & 5.0 & - & - & + & - \\
\hline $53.2-1-3,22$ & $<0.001$ & $<1.0$ & - & - & + & - \\
\hline $53.2-1-5,7$ & $<0.001$ & 1.0 & - & - & + & - \\
\hline $53.2-5-\mathrm{CC}$ & $<0.001$ & 12.0 & $<1.0$ & - & + & - \\
\hline $54.0-1-3,35$ & $<0.001$ & $<1.0$ & $<1.0$ & - & - & - \\
\hline $54.0-2-2,23$ & $<0.001$ & 1.0 & $<1.0$ & - & - & - \\
\hline $54.0-4-1,108$ & $<0.001$ & 1.0 & - & - & - & - \\
\hline $54.0-6-2,16$ & $<0.001$ & $<1.0$ & - & - & - & - \\
\hline $54.0-6-4,16$ & $<0.001$ & 1.0 & $<1.0$ & - & - & - \\
\hline $55.0-2-5,7$ & $<0.001$ & - & 100.0 & - & - & - \\
\hline $55.0-4-6,145$ & $<0.001$ & - & 100.0 & - & - & - \\
\hline $55.0-6-5,16$ & $<0.001$ & 1.0 & 98.0 & - & - & - \\
\hline $55.0-7-3,18$ & $<0.001$ & - & 100.0 & - & - & - \\
\hline
\end{tabular}


TABLE 5 - Continued

\begin{tabular}{lcccccc}
\hline \multicolumn{1}{c}{ Sample No. } & Fraction $(\mathrm{mm})$ & Quartz $\%$ & Calcite $\%$ & Aragonite $\%$ & Feldspar & Cristobalite \\
\hline $56.2-8-2,17$ & $<0.001$ & - & 100.0 & - & - & - \\
$57.1-1-2,0$ & $<0.001$ & - & 100.0 & - & - & - \\
$57.1-4-5,145$ & $<0.001$ & 32.0 & - & - & + & - \\
$57.1-2-1,44$ & $<0.001$ & 1.0 & 96.0 & - & - & - \\
$60.0-4-C C$ & $<0.001$ & - & 5.0 & - & - & - \\
\hline
\end{tabular}


TABLE 6

X-Ray Analysis of Clay Minerals in Fraction $<0.001 \mathrm{~mm}$

\begin{tabular}{|c|c|c|c|c|c|c|c|c|c|c|c|c|c|c|}
\hline \multirow{3}{*}{ Sample No. } & \multicolumn{6}{|c|}{ Major Minerals } & \multicolumn{6}{|c|}{ Minor Minerals (10\%) } & \multirow{3}{*}{$\begin{array}{c}\text { Removal of } \\
\mathrm{KCO}_{3}\end{array}$} & \multirow{3}{*}{$\begin{array}{c}\text { Abundance } \\
\text { Amorphous } \\
\text { Material }\end{array}$} \\
\hline & \multicolumn{2}{|l|}{ Illite } & \multicolumn{2}{|c|}{ Chlorite } & \multicolumn{2}{|c|}{ Montmorillonite } & \multirow[t]{2}{*}{ Illite } & \multirow[t]{2}{*}{ Kaolinite } & \multirow[t]{2}{*}{ Chlorite } & \multirow[t]{2}{*}{$\begin{array}{c}\text { Montmor- } \\
\text { illonite }\end{array}$} & \multirow[t]{2}{*}{$\begin{array}{l}\text { Mixed } \\
\text { Layer }\end{array}$} & \multirow[t]{2}{*}{$\begin{array}{c}\text { Paly- } \\
\text { gorskite }\end{array}$} & & \\
\hline & $\begin{array}{c}4 \times \text { area } \\
\left(\mathrm{cm}^{2}\right)\end{array}$ & $\%$ & $\begin{array}{c}2 \times \text { area } \\
\left(\mathrm{cm}^{2}\right)\end{array}$ & $\%$ & $\begin{array}{c}1 \times \text { area } \\
\left(\mathrm{cm}^{2}\right)\end{array}$ & $\%$ & & & & & & & & \\
\hline $45.1-1-1,7$ & 406 & 24 & 140 & 8 & 1120 & 68 & - & - & - & - & + & - & + & + \\
\hline $45.1-1-2,100$ & - & - & - & - & - & 100 & + & - & + & - & - & - & + & + \\
\hline $47.0-1-4,90$ & 356 & 69 & 150 & 31 & - & - & - & + & - & - & + & + & + & + \\
\hline $47.2-1-2,23$ & 462 & 81 & 110 & 13 & - & - & - & - & - & - & + & + & - & + \\
\hline $47.2-1-3,90$ & 276 & 72 & 105 & 28 & - & - & - & + & - & - & + & + & + & + \\
\hline $47.2-9-6,0$ & 392 & 34 & - & - & 770 & 66 & - & - & + & - & + & + & - & + \\
\hline $47.2-11-4,145$ & 312 & 32 & 40 & 48 & - & - & - & - & - & - & + & + & - & + \\
\hline $49.0-1-1,11$ & 570 & 74 & 185 & 26 & - & - & - & - & - & - & - & + & - & + \\
\hline $49.0-1-2,46$ & 780 & 74 & 270 & 26 & - & - & - & - & - & - & - & - & + & + \\
\hline $49.0-1-4,6$ & 240 & 61 & 154 & 32 & - & - & - & - & - & + & - & + & + & + \\
\hline $49.0-1-5,70$ & 840 & 59 & 240 & 17 & 340 & 24 & - & - & - & - & - & + & + & + \\
\hline $49.1-1-1,77$ & 476 & 80 & 120 & 20 & - & - & - & - & - & - & - & - & + & + \\
\hline $49.1-1-2,124$ & 420 & 37 & 120 & 10 & 600 & 53 & - & - & - & - & - & - & + & + \\
\hline $49.1-1-4,17$ & - & - & - & - & - & 100 & + & - & + & - & - & + & + & + \\
\hline $50.0-2-1,145$ & 294 & 59 & - & - & 200 & 41 & - & - & - & - & + & + & - & + \\
\hline $50.0-2-2,150$ & 132 & 50 & - & - & 130 & 50 & - & - & + & - & + & + & - & + \\
\hline $50.0-2-3,150$ & 280 & 31 & - & - & 620 & 69 & - & - & + & - & + & + & - & + \\
\hline $50.0-2-4,150$ & 200 & 31 & - & - & 540 & 69 & - & - & + & - & + & + & - & + \\
\hline $50.0-2-6,150$ & 200 & 40 & - & - & 300 & 60 & - & - & + & - & + & + & - & + \\
\hline $50.1-3-4,16$ & 220 & 65 & 120 & 35 & - & - & - & - & - & + & - & + & + & + \\
\hline $50.1-3-6,16$ & 1040 & 61 & 203 & 12 & 441 & 27 & - & + & - & - & - & + & + & + \\
\hline $50.1-4-1,75$ & 354 & 50 & 70 & 10 & 280 & 40 & - & + & - & - & + & + & + & + \\
\hline $51.0-1-6,16$ & 207 & 36 & 72 & 12 & 300 & 52 & - & + & - & - & + & + & + & + \\
\hline
\end{tabular}


TABLE 6 - Continued

\begin{tabular}{|c|c|c|c|c|c|c|c|c|c|c|c|c|c|c|}
\hline \multirow{3}{*}{ Sample No. } & \multicolumn{6}{|c|}{ Major Minerals } & \multicolumn{6}{|c|}{ Minor Minerals ( $10 \%)$} & \multirow{3}{*}{$\begin{array}{c}\text { Removal of } \\
\mathrm{KCO}_{3}\end{array}$} & \multirow{3}{*}{$\begin{array}{l}\text { Abundance } \\
\text { Amorphous } \\
\text { Material }\end{array}$} \\
\hline & \multicolumn{2}{|l|}{ Illite } & \multicolumn{2}{|c|}{ Chlorite } & \multicolumn{2}{|c|}{ Montmorillonite } & \multirow[t]{2}{*}{ Illite } & \multirow[t]{2}{*}{ Kaolinite } & \multirow[t]{2}{*}{ Chlorite } & \multirow[t]{2}{*}{$\begin{array}{l}\text { Montmor- } \\
\text { illonite }\end{array}$} & \multirow[t]{2}{*}{$\begin{array}{l}\text { Mixed } \\
\text { Layer }\end{array}$} & \multirow[t]{2}{*}{$\begin{array}{c}\text { Paly- } \\
\text { gorskite }\end{array}$} & & \\
\hline & $\begin{array}{c}4 \times \text { area } \\
\left(\mathrm{cm}^{2}\right)\end{array}$ & $\%$ & $\begin{array}{c}2 \times \text { area } \\
\left(\mathrm{cm}^{2}\right)\end{array}$ & $\%$ & $\begin{array}{c}1 \times \text { area } \\
\left(\mathrm{cm}^{2}\right)\end{array}$ & $\%$ & & & & & & & & \\
\hline $52.0-1-4,8$ & 320 & 39 & 120 & 13 & 450 & 48 & - & + & - & - & - & - & + & + \\
\hline $52.0-1-6,9$ & - & - & - & - & - & 100 & + & + & + & - & - & \pm & + & - \\
\hline $52.0-2-3,7$ & - & - & - & - & - & 100 & + & + & + & - & - & - & + & - \\
\hline $52.0-2-5,18$ & - & - & - & - & - & 100 & + & + & + & - & - & - & + & - \\
\hline $52.0-3-1,16$ & - & - & - & - & - & 100 & + & - & + & - & - & - & + & - \\
\hline $52.0-3-3,16$ & - & - & - & - & - & 100 & + & - & - & - & - & - & + & - \\
\hline $52.0-3-5,7$ & - & - & - & - & - & 100 & + & + & + & - & - & - & + & - \\
\hline $52.0-4-2,7$ & - & - & - & - & - & 100 & + & + & + & - & - & - & + & - \\
\hline $52.0-4-4,6$ & - & - & - & - & - & 100 & + & + & + & - & - & - & + & - \\
\hline $52.0-4-6,18$ & 660 & 27 & 228 & 9 & 1560 & 64 & - & + & - & - & - & - & + & - \\
\hline $52.0-5-1,127$ & 916 & 30 & 90 & 12 & 420 & 58 & - & + & - & - & - & - & + & - \\
\hline $52.0-5-2,17$ & 420 & 40 & 126 & 10 & 410 & 50 & - & + & - & - & - & - & + & - \\
\hline $55.0-1-2$ & 180 & 72 & 70 & 28 & - & - & - & + & - & - & + & - & - & + \\
\hline $58.2-1-3$ & - & - & - & - & - & 100 & - & - & - & - & - & - & - & ++ \\
\hline $58.2-1-5$ & - & - & - & - & - & 100 & - & - & + & - & - & - & - & ++ \\
\hline $60.0-1-1$ & - & - & - & - & & 100 & - & - & - & - & - & - & + & + \\
\hline $60.0-2-1$ & - & - & 225 & 39 & 348 & 61 & - & - & - & - & + & - & + & + \\
\hline $60.0-3-2$ & - & - & 180 & 27 & 480 & 73 & - & + & - & - & - & - & + & + \\
\hline $60.0-5-1$ & - & - & - & - & - & 100 & + & - & + & - & - & - & + & + \\
\hline $60.0-5-3$ & - & - & - & - & - & 100 & - & - & + & - & - & - & - & + \\
\hline $60.0-6-2$ & - & - & 60 & 37 & 100 & 63 & - & - & - & - & - & - & + & + \\
\hline $60.0-6-4$ & - & - & - & - & - & 100 & - & - & + & - & - & - & + & + \\
\hline $60.0-6-6$ & - & - & - & - & - & 100 & - & - & + & - & - & - & + & + \\
\hline $60.0-7-1$ & - & - & - & - & - & 100 & - & - & + & - & - & - & + & + \\
\hline $60.0-8-1$ & - & - & - & - & - & 100 & - & - & + & - & - & - & + & + \\
\hline $60.0-8-3$ & - & - & - & - & - & 100 & - & - & + & - & - & - & + & + \\
\hline
\end{tabular}


TABLE 7

Results of Partial Silicate Analysis

\begin{tabular}{|c|c|c|c|c|c|c|c|c|c|c|c|}
\hline Sample Designation & $\begin{array}{c}\mathrm{SiO}_{2} \\
(\%)\end{array}$ & $\begin{array}{l}\mathrm{SiO}_{2} / \\
\mathrm{Al}_{2} \mathrm{O}_{3}\end{array}$ & $\begin{array}{c}\mathrm{Al}_{2} \mathrm{O}_{3} \\
(\%)\end{array}$ & $\begin{array}{c}\mathrm{Al}_{2} \mathrm{O}_{3} / \\
\mathrm{TiO}_{2}\end{array}$ & $\begin{array}{l}\mathrm{CaO} \\
(\%)\end{array}$ & $\begin{array}{l}\mathrm{MgO} \\
(\%)\end{array}$ & $\mathrm{CaO} / \mathrm{MgO}$ & $\begin{array}{c}\mathrm{MnO} \\
(\%)\end{array}$ & $\begin{array}{c}\mathrm{TiO}_{2} \\
(\%)\end{array}$ & $\begin{array}{c}\mathrm{Fe}_{2} \mathrm{O}_{3} / \\
\mathrm{TiO}_{2}\end{array}$ & $\begin{array}{c}\mathrm{Fe}_{2} \mathrm{O}_{3} \\
(\%)\end{array}$ \\
\hline $44.0-1-3,145$ & $<4.60$ & & $<0.70$ & & 50.70 & $<0.66$ & & $<0.030$ & $<0.15$ & & $<1.90$ \\
\hline $44.0-1-1,145$ & $<4.60$ & & $<0.70$ & & 56.20 & $<0.66$ & & $<0.030$ & $<0.15$ & & $<1.90$ \\
\hline $44.0-1-2,145$ & $<4.60$ & & $<0.70$ & & 56.80 & $<0.66$ & & $<0.030$ & $<0.15$ & & $<1.90$ \\
\hline $44.0-1-3,145$ & $<4.60$ & & $<0.70$ & & 48.40 & $<0.66$ & & $<0.030$ & $<0.15$ & & $<1.90$ \\
\hline 44.0-1-3, All slops & $<4.60$ & & $<0.70$ & & 51.90 & $<0.66$ & & $<0.030$ & $<0.15$ & & $<1.90$ \\
\hline $44.0-1-4,145$ & $<4.60$ & & $<0.70$ & & 50.70 & $<0.66$ & & $<0.030$ & $<0.15$ & & $<1.90$ \\
\hline $44.0-2-\mathrm{CC}$ & $<4.60$ & & $<0.70$ & & 38.90 & $<0.66$ & & $<0.030$ & $<0.15$ & & $<1.90$ \\
\hline $44.0-2-1,145$ & $<4.60$ & & $<0.70$ & & 47.80 & $<0.66$ & & $<0.030$ & $<0.15$ & & $<1.90$ \\
\hline $44.0-2-2,145$ & $<4.60$ & & $<0.70$ & & 46.30 & $<0.66$ & & 0.030 & $<0.15$ & & $<1.90$ \\
\hline $44.0-2-3,145$ & $<4.60$ & & $<0.70$ & & 50.20 & $<0.66$ & & $<0.030$ & $<0.15$ & & $<1.90$ \\
\hline $44.0-2-4,145$ & $<4.60$ & & $<0.70$ & & 47.30 & $<0.66$ & & $<0.030$ & $<0.15$ & & $<1.90$ \\
\hline $44.0-2-5,145$ & $<4.60$ & & $<0.70$ & & 46.30 & $<0.66$ & & $<0.030$ & $<0.15$ & & $<1.90$ \\
\hline $44.0-2-6,145$ & $<4.60$ & & $<0.70$ & & 47.90 & $<0.66$ & & $<0.030$ & $<0.15$ & & $<1.90$ \\
\hline $44.0-3-\mathrm{CC}$ & 12.70 & & $<0.70$ & & 43.60 & $<0.66$ & & 0.090 & $<0.15$ & & $<0.190$ \\
\hline $44.0-3-2,145$ & $<4.60$ & & $<0.70$ & & 48.40 & $<0.66$ & & 0.031 & $<0.15$ & & $<1.90$ \\
\hline $44.0-3-3,145$ & $<4.60$ & & $<0.70$ & & 51.80 & $<0.66$ & & 0.031 & $<0.15$ & & $<1.90$ \\
\hline $44.0-3-4,145$ & $<4.60$ & & $<0.70$ & & 48.40 & $<0.66$ & & 0.037 & $<0.15$ & & $<1.90$ \\
\hline $44.0-3-5,145$ & 10.20 & & $<0.70$ & & 45.20 & $<0.66$ & & 0.040 & $<0.15$ & & $<1.90$ \\
\hline 44.0-4-0, Tор & $>93.50$ & & 2.82 & & 0.88 & $<0.66$ & & $<0.030$ & $<0.15$ & & $<1.90$ \\
\hline $44.0-4-2,145$ & $<4.60$ & & $<0.70$ & & 51.90 & $<0.66$ & & 0.038 & $<0.15$ & & $<1.90$ \\
\hline $44.0-4-3,145$ & $<4.60$ & & $<0.70$ & & 56.20 & $<0.66$ & & 0.035 & $<0.15$ & & $<1.90$ \\
\hline $44.0-4-4,145$ & $<4.60$ & & $<0.70$ & & 55.00 & $<0.66$ & & 0.033 & $<0.15$ & & $<1.90$ \\
\hline $44.0-4-5,145$ & $<4.60$ & & $<0.70$ & & 47.30 & $<0.66$ & & 0.049 & $<0.15$ & & $<1.90$ \\
\hline $44.0-4-6,145$ & $<4.60$ & & $<0.70$ & & 59.60 & $<0.66$ & & 0.044 & $<0.15$ & & $<1.90$ \\
\hline $44.0-4-\mathrm{CC}$ & $<4.60$ & & $<0.70$ & & 58.90 & $<0.66$ & & 0.038 & $<0.15$ & & $<1.90$ \\
\hline 44.0-5-0, Total & 11.20 & & $<0.70$ & & 50.70 & $<0.66$ & & 0.040 & $<0.15$ & & 3.59 \\
\hline $44.1-1-\mathrm{CC}$ & 38.80 & 3.13 & 12.40 & 12.16 & 3.80 & 5.69 & 0.67 & $>0.210$ & 1.02 & 8.33 & 8.50 \\
\hline $45.1-1-1,7$ & 42.60 & 3.16 & 13.50 & 10.47 & 2.82 & 2.95 & 0.96 & $>0.210$ & 1.29 & 7.16 & 9.23 \\
\hline $45.1-1-1,70$ & 48.40 & 3.81 & 12.70 & 7.05 & 1.78 & 1.20 & 1.48 & $>0.210$ & 1.80 & 5.83 & 10.50 \\
\hline $45.1-1-1,145$ & 38.80 & 3.23 & 12.00 & 5.7 & 3.27 & 3.20 & 1.02 & $>0.210$ & 2.10 & & $>10.00$ \\
\hline $45.1-1-2,100$ & 41.70 & 3.02 & 13.80 & 7.93 & 2.75 & 3.47 & 0.79 & $>0.210$ & 1.74 & & $>10.00$ \\
\hline $45.1-1-2,124$ & 44.60 & 3.81 & 11.70 & & 6.31 & 3.77 & 1.67 & 0.210 & $>2.25$ & & $>10.00$ \\
\hline $45.1-1-3,23$ & 43.60 & 3.73 & 11.70 & & 5.90 & 5.25 & 1.12 & 0.150 & $>2.25$ & & $>10.00$ \\
\hline $45.1-1-4,22$ & 40.80 & 4.08 & 10.00 & & 6.92 & 5.25 & 1.32 & 0.170 & $>2.25$ & & $>10.00$ \\
\hline $45.1-1-4,136$ & 34.30 & 3.69 & 9.30 & 4.45 & 14.80 & 5.25 & 2.82 & 0.140 & 2.09 & 4.41 & 9.20 \\
\hline $45.1-1-5,4$ & 42.20 & 2.37 & 17.80 & 8.9 & 11.00 & 6.60 & 1.67 & $>0.210$ & 2.00 & 5.00 & 10.00 \\
\hline $45.1-3-\mathrm{CC}$ & 70.80 & 14.45 & 4.90 & 14. & 0.91 & 1.51 & 0.60 & 0.210 & 0.35 & 6.34 & 2.22 \\
\hline $45.1-3-\mathrm{CC}$ & 91.00 & 12.18 & 4.47 & 17.19 & $<0.88$ & 1.10 & & 0.120 & 0.26 & 8.23 & 2.14 \\
\hline $45.1-3-\mathrm{CC}$ & 72.50 & 29.47 & 2.46 & & 6.03 & 0.72 & 8.38 & $<0.030$ & $<0.15$ & & $<1.90$ \\
\hline 46.0 & 91.20 & & $<0.70$ & & $<0.88$ & $<0.66$ & & $<0.030$ & $<0.15$ & & $<1.90$ \\
\hline $46.0-1-6$ & 79.40 & 33.79 & 2.35 & & $<0.88$ & $<0.66$ & & $<0.030$ & $<0.15$ & & $<1.90$ \\
\hline $46.0-1-6$ & 89.10 & & $<0.70$ & & $<0.88$ & $<0.66$ & & $<0.030$ & $<0.15$ & & $<1.90$ \\
\hline $46.0-1-1,145$ & 50.70 & 3.43 & 14.80 & & 2.10 & 3.98 & 0.60 & $>0.210$ & 0.62 & 23.87 & 6.46 \\
\hline $46.0-1-2,145$ & 49.60 & 3.35 & 14.80 & & $<0.88$ & 3.35 & & $>0.210$ & 0.77 & 19.22 & 6.84 \\
\hline $46.0-1-3,145$ & 45.70 & 3.69 & 12.40 & & 0.88 & 3.63 & 0.24 & $>0.210$ & 0.65 & 19.80 & 5.43 \\
\hline $46.0-1-4,145$ & 43.60 & 3.55 & 12.30 & & 4.63 & 2.92 & 1.59 & $>0.210$ & 0.76 & 16.18 & 6.61 \\
\hline
\end{tabular}


TABLE 7 - Continued

\begin{tabular}{|c|c|c|c|c|c|c|c|c|c|c|c|}
\hline Sample Designation & $\begin{array}{l}\mathrm{SiO}_{2} \\
(\%)\end{array}$ & $\begin{array}{c}\mathrm{SiO}_{2} / \\
\mathrm{Al}_{2} \mathrm{O}_{3}\end{array}$ & $\begin{array}{c}\mathrm{Al}_{2} \mathrm{O}_{3} \\
(\%)\end{array}$ & $\begin{array}{c}\mathrm{Al}_{2} \mathrm{O}_{3} / \\
\mathrm{TiO}_{2}\end{array}$ & $\begin{array}{l}\mathrm{CaO} \\
(\%)\end{array}$ & $\begin{array}{l}\mathrm{MgO} \\
(\%)\end{array}$ & $\mathrm{CaO} / \mathrm{MgO}$ & $\begin{array}{l}\mathrm{MnO} \\
(\%)\end{array}$ & $\begin{array}{c}\mathrm{TiO}_{2} \\
(\%)\end{array}$ & $\begin{array}{c}\mathrm{Fe}_{2} \mathrm{O}_{3} / \\
\mathrm{TiO}_{2}\end{array}$ & $\begin{array}{c}\mathrm{Fe}_{2} \mathrm{O}_{3} \\
(\%)\end{array}$ \\
\hline $46.0-1-5,145$ & 54.30 & 6.02 & 9.02 & & $<0.88$ & 2.57 & & $>0.210$ & 0.60 & 15.30 & 5.19 \\
\hline $46.0-1-6,145$ & 47.30 & 3.97 & 11.90 & 22.88 & 1.62 & 3.17 & 0.50 & $>0.210$ & 0.52 & 11.35 & 5.90 \\
\hline $46.1-2-\mathrm{CC}$ & 66.10 & 14.79 & 4.47 & 12.08 & 4.47 & 1.30 & 3.28 & 0.240 & 0.37 & 6.00 & 2.22 \\
\hline $47.0-1-1,17$ & 21.40 & 3.47 & 6.16 & 16.65 & 32.30 & 1.05 & 30.76 & 0.040 & 0.37 & 7.43 & 2.75 \\
\hline $47.0-1-2,5$ & 25.70 & 3.62 & 7.09 & 3.55 & 24.80 & 1.82 & 13.63 & 0.060 & 2.00 & 1.84 & 3.68 \\
\hline $47.0-1-2,16$ & 19.00 & 2.44 & 7.78 & 21.60 & 25.20 & 1.93 & 13.06 & 0.047 & 0.36 & 7.22 & 2.80 \\
\hline $47.0-1-3,16$ & 19.00 & & 7.78 & & 25.20 & 1.93 & & 0.047 & 0.36 & & 2.60 \\
\hline $47.0-1-4,90$ & 20.90 & 3.63 & 5.76 & 16.94 & 31.60 & 0.93 & 33.98 & 0.035 & 0.34 & 7.74 & 2.63 \\
\hline $47.0-1-4,95$ & 23.20 & 3.51 & 6.61 & 28.76 & 32.30 & 1.45 & 22.28 & 0.040 & 0.23 & 8.48 & 1.95 \\
\hline $47.0-1-5,3.5$ & 11.60 & 3.02 & 3.84 & 1.84 & 29.50 & 1.10 & 26.81 & 0.046 & 2.09 & 0.90 & $<1.90$ \\
\hline $47.0-1-6,14$ & 12.30 & 3.47 & 3.54 & 14.75 & 45.70 & $<0.66$ & & 0.042 & 0.24 & 8.13 & 1.95 \\
\hline 47.1-1, Top & $<7.60$ & & $<0.70$ & & 51.90 & $<0.66$ & & 0.080 & $<0.15$ & & $<1.90$ \\
\hline $47.1-1-, 145$ & $<7.60$ & & $<0.70$ & & 35.50 & 0.08 & & $<0.660$ & $<0.15$ & & $<1.90$ \\
\hline $47.1-1-2,16$ & $<4.60$ & & $<0.70$ & & 44.60 & $<0.66$ & & 0.058 & $<0.15$ & & $<1.90$ \\
\hline $47.1-3-1,17$ & $<4.60$ & & $<7.00$ & & 44.70 & 0.89 & & 0.090 & 0.15 & & $<1.90$ \\
\hline $47.2-1-2,23$ & 14.80 & 3.47 & 4.26 & 14.69 & 39.80 & 0.66 & & 0.051 & 0.29 & 7.90 & 2.29 \\
\hline $47.2-1-2,181$ & 12.20 & 2.74 & 4.26 & 1.35 & 43.10 & 1.11 & 38.83 & 0.050 & 0.33 & 0.69 & 2.29 \\
\hline $47.2-1-3,11$ & 13.50 & 1.76 & 7.68 & 27.43 & 33.90 & 0.94 & 36.06 & 0.058 & 0.28 & 7.82 & 2.19 \\
\hline $47.2-1-3,90$ & 8.51 & 2.34 & 3.63 & 16.50 & 45.20 & $<0.66$ & & 0.047 & 0.23 & & $<1.90$ \\
\hline $47.2-1-4,7$ & 10.00 & 3.72 & 2.69 & & 46.80 & 0.85 & 55.06 & 0.049 & $<0.15$ & & $<1.90$ \\
\hline $47.2-2-2,8$ & 12.60 & 3.20 & 3.94 & 15.76 & 42.60 & 0.90 & 47.33 & 0.043 & 0.25 & 8.36 & 2.09 \\
\hline $47.2-2-2,47$ & 16.60 & 4.46 & 3.72 & 12.00 & 42.20 & 1.51 & 27.95 & 0.056 & 0.31 & 7.23 & $2.24 ?$ \\
\hline $47.2-2-3,7$ & $<4.60$ & & 0.98 & & 52.60 & 0.60 & 87.67 & 0.100 & $<0.15$ & & $<1.90$ \\
\hline $47.2-2-4,7$ & 10.00 & 4.46 & 2.24 & 9.74 & 38.40 & $<0.66$ & & 0.038 & 0.23 & & $<1.90$ \\
\hline $47.2-2-5,5$ & $<4.60$ & & 0.83 & & 47.80 & 0.66 & 72.42 & 0.069 & $<0.15$ & & $<1.90$ \\
\hline $47.2-2-6,6$ & 4.57 & 3.81 & 1.20 & & 46.20 & $<0.66$ & & 0.076 & $<1.90$ & & $<0.15$ \\
\hline $47.2-3-2,6.5$ & 7.15 & 4.02 & 1.78 & & 35.90 & 0.87 & 41.26 & 0.036 & $<0.15$ & & 1.90 \\
\hline $47.2-3-3,6$ & 5.25 & 4.02 & 0.70 & & 50.00 & $<0.66$ & & 0.035 & $<0.15$ & & $<1.90$ \\
\hline $47.2-3-4,16$ & $<4.60$ & & $<0.70$ & & 40.40 & 0.63 & 64.12 & 0.090 & $<0.15$ & & $<1.90$ \\
\hline $47.2-3-\mathrm{CC}$ & $<4.60$ & & $<0.70$ & & 50.50 & $<0.66$ & & 0.062 & $<0.15$ & & $<1.90$ \\
\hline 47.2-4-CC & $<4.60$ & & $<0.70$ & & 41.70 & 0.89 & & 0.091 & $<0.07$ & & $<1.90$ \\
\hline $47.2-4-1,16$ & $<4.60$ & & $<0.70$ & & 49.00 & $<0.66$ & & 0.061 & $<0.15$ & & $<1.90$ \\
\hline $47.2-4-2,17$ & $<4.60$ & & $<0.70$ & & 51.40 & $<0.66$ & & 0.061 & $<0.15$ & & $<1.90$ \\
\hline $47.2-4-3,16$ & $<4.60$ & & $<0.70$ & & 47.30 & $<0.66$ & & 0.078 & $<0.15$ & & $<1.90$ \\
\hline $47.2-4-4,16.5$ & $<4.60$ & & $<0.70$ & & 37.20 & 0.57 & 65.26 & 0.046 & $<0.15$ & & $<1.90$ \\
\hline $47.2-4-5,16.5$ & $<4.60$ & & $<0.70$ & & 53.60 & $<0.66$ & & 0.055 & $<0.15$ & & $<1.90$ \\
\hline $47.2-4-6,17$ & $<4.60$ & & $<0.70$ & & 50.60 & $<0.66$ & & 0.097 & $<0.15$ & & $<1.90$ \\
\hline $47.2-5-1,17$ & $<4.60$ & & $<0.70$ & & 50.00 & $<0.66$ & & 0.076 & $<0.15$ & & $<1.90$ \\
\hline $47.2-5-2,6$ & $<4.60$ & & $<0.70$ & & 49.50 & $<0.66$ & & 0.082 & $<0.15$ & & $<1.90$ \\
\hline $47.2-5-3,6$ & $<4.60$ & & $<0.70$ & & 48.40 & $<0.66$ & & 0.074 & $<0.15$ & & $<1.90$ \\
\hline $47.2-5-4,6$ & $<4.60$ & & 1.00 & & 38.90 & 0.60 & 64.83 & 0.068 & $<0.15$ & & $<1.90$ \\
\hline $47.2-5-5,6$ & $<4.60$ & & $<0.70$ & & 43.20 & $<0.66$ & & 0.071 & $<0.15$ & & $<1.90$ \\
\hline $47.2-5-6,6.5$ & $<4.60$ & & $<0.70$ & & 41.60 & 0.56 & 74.29 & 0.078 & $<0.15$ & & $<1.90$ \\
\hline $47.2-6-2,6$ & $<4.60$ & & $<0.70$ & & 51.30 & $<0.66$ & & 0.091 & $<0.15$ & & $<1.90$ \\
\hline $47.2-6-3,17$ & $<4.60$ & & $<0.70$ & & 49.50 & $<0.66$ & & 0.090 & $<0.15$ & & $<1.90$ \\
\hline $47.2-7-1,17$ & $<4.60$ & & $<0.70$ & & 49.00 & $<0.66$ & & 0.148 & $<0.15$ & & $<1.90$ \\
\hline $47.2-7-2,17$ & $<4.60$ & & $<0.70$ & & 45.20 & $<0.66$ & & 0.054 & $<0.15$ & & $<1.90$ \\
\hline $47.2-7-3,13$ & $<4.60$ & & $<0.70$ & & 52.50 & $<0.66$ & & 0.085 & $<0.15$ & & $<1.90$ \\
\hline
\end{tabular}


TABLE 7 - Continued

\begin{tabular}{|c|c|c|c|c|c|c|c|c|c|c|c|}
\hline Sample Designation & $\begin{array}{c}\mathrm{SiO}_{2} \\
(\%)\end{array}$ & $\begin{array}{l}\mathrm{SiO}_{2} / \\
\mathrm{Al}_{2} \mathrm{O}_{3}\end{array}$ & $\begin{array}{c}\mathrm{Al}_{2} \mathrm{O}_{3} \\
(\%)\end{array}$ & $\begin{array}{c}\mathrm{Al}_{2} \mathrm{O}_{3} / \\
\mathrm{TiO}_{2}\end{array}$ & $\begin{array}{l}\mathrm{CaO} \\
(\%)\end{array}$ & $\begin{array}{l}\mathrm{MgO} \\
(\%)\end{array}$ & $\mathrm{CaO} / \mathrm{MgO}$ & $\begin{array}{c}\mathrm{MnO} \\
(\%)\end{array}$ & $\begin{array}{c}\mathrm{TiO}_{2} \\
(\%)\end{array}$ & $\begin{array}{c}\mathrm{Fe}_{2} \mathrm{O}_{3} / \\
\mathrm{TiO}_{2}\end{array}$ & $\begin{array}{c}\mathrm{Fe}_{2} \mathrm{O}_{3} \\
(\%)\end{array}$ \\
\hline $47.2-7-4,17$ & $<4.60$ & & $<0.70$ & & 55.00 & $<0.66$ & \multirow{49}{*}{0.10} & 0.057 & $<0.15$ & & $<1.90$ \\
\hline $47.2-7-5,27.5$ & $<4.60$ & & $<0.70$ & & 58.80 & $<0.66$ & & 0.042 & 0.15 & & $<1.90$ \\
\hline $47.2-7-6,126$ & $<4.60$ & & $<0.70$ & & 49.00 & $<0.66$ & & 0.066 & $<0.15$ & & $<1.90$ \\
\hline $47.2-8-1,27$ & $<4.60$ & & $<0.70$ & & 56.20 & $<0.66$ & & 0.037 & $<0.15$ & & $<1.90$ \\
\hline $47.2-8-2,17$ & $<8.60$ & & $<0.70$ & & 48.40 & $<0.66$ & & 0.047 & $<0.15$ & & $<1.90$ \\
\hline $47.2-8-3,18$ & $<4.60$ & & $<0.70$ & & 56.20 & $<0.66$ & & 0.037 & $<0.15$ & & $<1.90$ \\
\hline $47.1-8-4,17$ & $<4.60$ & & $<0.70$ & & 46.30 & $<0.66$ & & 0.032 & $<0.15$ & & $<1.90$ \\
\hline $47.2-8-5,17$ & $<4.60$ & & $<0.70$ & & 47.80 & $<0.66$ & & $<0.030$ & $<0.15$ & & $<1.90$ \\
\hline $47.2-8-6,16$ & $<4.60$ & & $<0.70$ & & 52.50 & $<0.66$ & & 0.029 & $<0.15$ & & $<1.90$ \\
\hline $47.2-8-6-\mathrm{CC}$ & $<4.60$ & & $<0.70$ & & 59.00 & $<0.66$ & & 0.046 & $<0.15$ & & $<1.90$ \\
\hline $47.2-9-1,16$ & $<4.60$ & & $<0.70$ & & 55.60 & $<0.66$ & & 0.036 & $<0.15$ & & $<1.90$ \\
\hline $47.2-9-1,150$ & $<4.60$ & & $<0.70$ & & 45.60 & $<0.66$ & & 0.070 & $<0.15$ & & $<1.90$ \\
\hline $47.2-9-2,17$ & $<4.60$ & & $<0.70$ & & 47.30 & $<0.66$ & & 0.050 & $<0.15$ & & $<1.90$ \\
\hline $47.2-9-3,17$ & $<4.60$ & & $<0.70$ & & 56.20 & $<0.66$ & & 0.056 & $<0.15$ & & $<1.90$ \\
\hline $47.2-9-4,0$ & $<4.60$ & & $<0.70$ & & 43.60 & $<0.66$ & & 0.040 & $<0.15$ & & $<1.90$ \\
\hline $47.2-9-5,17$ & $<4.60$ & & $<0.70$ & & $>60.00$ & $<0.66$ & & 0.047 & $<0.15$ & & $<1.90$ \\
\hline $47.2-9-5,150$ & $<4.60$ & & $<0.70$ & & 42.60 & $<0.66$ & & 0.050 & $<0.15$ & & $<1.90$ \\
\hline $47.2-9-6,0$ & $<4.60$ & & $<0.70$ & & 50.20 & $<0.66$ & & 0.051 & $<0.15$ & & $<1.90$ \\
\hline 47.2-9-CC & $<4.60$ & & $<0.70$ & & 40.80 & $<0.66$ & & 0.120 & $<0.15$ & & $<1.90$ \\
\hline $47.2-10-1,17$ & $<4.60$ & & $<0.70$ & & 49.00 & $<0.66$ & & 0.032 & $<0.15$ & & $<1.90$ \\
\hline $47.2-10-2,17$ & $<4.60$ & & $<0.70$ & & 49.00 & $<0.66$ & & 0.030 & $<0.15$ & & $<1.90$ \\
\hline $47.2-10-3,18$ & $<4.60$ & & $<0.70$ & & 50.20 & $<0.66$ & & 0.036 & $<0.15$ & & $<1.90$ \\
\hline $47.2-10-4,17$ & $<4.60$ & & $<0.70$ & & 55.60 & $<0.66$ & & 0.100 & $<0.15$ & & $<1.90$ \\
\hline $47.2-10-5,17$ & $<4.60$ & & $<0.70$ & & $>60.00$ & $<0.66$ & & 0.100 & $<0.15$ & & $<1.90$ \\
\hline $47.2-10-6,16$ & $<4.60$ & & $<0.70$ & & 52.50 & $<0.66$ & & 0.045 & $<0.15$ & & $<1.90$ \\
\hline $47.2-10-\mathrm{CC}$ & $<4.60$ & & $<0.70$ & & 37.10 & $<0.66$ & & 0.060 & $<0.15$ & & $<1.90$ \\
\hline 47.2-10, Top & $<4.60$ & & $<0.70$ & & 51.30 & $<0.66$ & & 0.004 & $<0.15$ & & $<1.90$ \\
\hline $47.2-11-1,145$ & $<4.60$ & & $<0.70$ & & 46.80 & $<0.66$ & & 0.023 & $<0.15$ & & $<1.90$ \\
\hline $47.2-11-2,145$ & $<4.60$ & & $<0.70$ & & 57.60 & $<0.66$ & & $<0.030$ & $<0.15$ & & $<1.90$ \\
\hline $47.2-11-3,145$ & $<4.60$ & & $<0.70$ & & 50.00 & $<0.66$ & & $<0.030$ & $<0.15$ & & $<1.90$ \\
\hline $47.2-11-4,145$ & $<4.60$ & & $<0.70$ & & 47.30 & $<0.66$ & & $<0.030$ & $<0.15$ & & $<1.90$ \\
\hline $47.2-11-5,145$ & $<4.60$ & & $<0.70$ & & 57.60 & $<0.66$ & & 0.028 & $<0.15$ & & $<1.90$ \\
\hline $47.2-11-6,145$ & $<4.60$ & & $<0.70$ & & 38.00 & $<0.66$ & & $<0.030$ & $<0.15$ & & $<1.90$ \\
\hline $47.2-11-\mathrm{CC}$ & $<4.60$ & & $<0.70$ & & 57.60 & $<0.66$ & & $<0.030$ & $<0.15$ & & $<1.90$ \\
\hline $47.2-12-1,145$ & $<4.60$ & & $<0.70$ & & 53.70 & $<0.66$ & & $<0.030$ & $<0.15$ & & $<1.90$ \\
\hline $47.2-12-2,17$ & $<4.60$ & & $<0.70$ & & $<51.30$ & $<0.66$ & & $<0.030$ & $<0.15$ & & $<1.90$ \\
\hline $47.2-12-3,145$ & $<4.60$ & & $<0.70$ & & 46.90 & $<0.66$ & & $<0.030$ & $<0.15$ & & $<1.90$ \\
\hline $47.2-12-4,145$ & $<4.60$ & & $<0.70$ & & 59.00 & $<0.66$ & & $<0.030$ & $<0.15$ & & $<1.90$ \\
\hline $47.2-12-\mathrm{CC}$ & $<4.60$ & & $<0.70$ & & 56.30 & $<0.66$ & & $<0.030$ & $<0.15$ & & $<1.90$ \\
\hline $47.2-13-1,145$ & $<4.60$ & & $<0.70$ & & 57.60 & $<0.66$ & & $<0.030$ & $<0.15$ & & $<1.90$ \\
\hline $47.2-13-2,145$ & $<4.60$ & & $<0.70$ & & 52.50 & $<0.66$ & & $<0.030$ & $<0.15$ & & $<1.90$ \\
\hline $47.2-13-3,16$ & $<4.60$ & & $<0.70$ & & 45.20 & $<0.66$ & & $<0.030$ & $<0.15$ & & $<1.90$ \\
\hline $47.2-13-4,145$ & $<4.60$ & & $<0.70$ & & 57.60 & $<0.66$ & & 0.027 & $<0.15$ & & $<1.90$ \\
\hline $47.2-13-5,145$ & $<4.60$ & & $<0.70$ & & 49.60 & $<0.66$ & & $<0.030$ & $<0.15$ & & $<1.90$ \\
\hline $47,2-13-6,145$ & $<4.60$ & & $<0.70$ & & 54.30 & $<0.66$ & & $<0.030$ & $<0.15$ & & $<1.90$ \\
\hline $47.2-13-\mathrm{CC}$ & $<4.60$ & & $<0.70$ & & 46.80 & $<0.66$ & & $<0.040$ & $<0.15$ & & $<1.90$ \\
\hline $47.2-14-1,145$ & $<4.60$ & & $<0.70$ & & 55.00 & $<0.66$ & & $<0.039$ & $<0.15$ & & $<1.90$ \\
\hline $47.2-14-2,145$ & $<4.60$ & & $<0.70$ & & 49.00 & $<0.66$ & & $<0.030$ & $<0.15$ & & $<1.90$ \\
\hline $47.2-14-3,145$ & $<4.60$ & & $<0.70$ & & 52.50 & $<0.66$ & & $<0.030$ & $<0.15$ & & $<1.90$ \\
\hline
\end{tabular}


TABLE 7 - Continued

\begin{tabular}{|c|c|c|c|c|c|c|c|c|c|c|c|}
\hline Sample Designation & $\begin{array}{c}\mathrm{SiO}_{2} \\
(\%)\end{array}$ & $\begin{array}{l}\mathrm{SiO}_{2} / \\
\mathrm{Al}_{2} \mathrm{O}_{3}\end{array}$ & $\begin{array}{c}\mathrm{Al}_{2} \mathrm{O}_{3} \\
(\%)\end{array}$ & $\begin{array}{c}\mathrm{Al}_{2} \mathrm{O}_{3} / \\
\mathrm{TiO}_{2}\end{array}$ & $\begin{array}{l}\mathrm{CaO} \\
(\%)\end{array}$ & $\begin{array}{r}\mathrm{MgO} \\
(\%)\end{array}$ & $\mathrm{CaO} / \mathrm{MgO}$ & $\begin{array}{r}\mathrm{MnO} \\
(\%)\end{array}$ & $\begin{array}{c}\mathrm{TiO}_{2} \\
(\%)\end{array}$ & $\begin{array}{c}\mathrm{Fe}_{2} \mathrm{O}_{3} / \\
\mathrm{TiO}_{2}\end{array}$ & $\begin{array}{c}\mathrm{Fe}_{2} \mathrm{O}_{3} \\
(\%)\end{array}$ \\
\hline $47.2-14-4,145$ & $<4.60$ & & $<0.70$ & & $>60.00$ & $<0.66$ & & $<0.030$ & $<0.15$ & & $<1.90$ \\
\hline $47.2-14-5,145$ & $<4.60$ & & $<0.70$ & & 50.60 & $<0.66$ & & $<0.030$ & $<0.15$ & & $<1.90$ \\
\hline $47.2-14-6,145$ & $<4.60$ & & $<0.70$ & & 57.60 & $<0.66$ & & $<0.030$ & $<0.15$ & & $<1.90$ \\
\hline $47.2-14-\mathrm{CC}$ & $<4.60$ & & $<0.70$ & & 56.30 & $<0.66$ & & $<0.030$ & $<0.15$ & & $<1.90$ \\
\hline $48.1-1-1,26$ & 10.10 & 0.78 & 3.23 & 19.00 & 50.50 & 0.98 & 51.53 & 0.170 & 0.17 & 10.47 & 1.78 \\
\hline $48.2-1-\mathrm{CC}$ & $<4.60$ & & $<0.70$ & & 55.00 & $<0.66$ & & 0.035 & $<0.15$ & & $<1.90$ \\
\hline $48.2-1-1,28$ & $<4.60$ & & $<0.70$ & & 45.60 & $<0.66$ & & 0.090 & $<0.15$ & & $<1.90$ \\
\hline $48.2-1-2,33$ & $<4.60$ & & $<0.70$ & & 49.50 & $<0.66$ & & 0.076 & $<0.15$ & & $<1.90$ \\
\hline $48.2-1-3,16$ & $<4.60$ & & $<0.70$ & & 42.60 & 0.65 & 65.54 & 0.076 & $<0.15$ & & $<1.90$ \\
\hline $48.2-1-4,16$ & $<4.60$ & & $<0.70$ & & 44.70 & $<0.66$ & & 0.058 & $<0.15$ & & $<1.90$ \\
\hline $48.2-1-5,14.5$ & $<4.60$ & & $<0.70$ & & 45.20 & $<0.60$ & 75.33 & 0.083 & $<0.15$ & & $<1.90$ \\
\hline $48.2-1-6,11$ & $<4.60$ & & $<0.70$ & & 53.60 & $<0.66$ & & $<0.030$ & $<0.15$ & & $<1.90$ \\
\hline $48.2-2-1,145$ & $<4.60$ & & $<0.70$ & & 44.20 & $<0.66$ & & $<0.030$ & $<0.15$ & & $<1.90$ \\
\hline $48.2-2-2,17$ & $<4.60$ & & $<0.70$ & & $>60.00$ & $<0.66$ & & $<0.030$ & $<0.15$ & & $<1.90$ \\
\hline $48.2-2-3,145$ & $<4.60$ & & $<0.70$ & & 57.60 & $<0.66$ & & $<0.030$ & $<0.15$ & & $<1.90$ \\
\hline $48.2-2-4,18$ & $<4.60$ & & $<0.70$ & & 57.60 & $<0.66$ & & $<0.030$ & $<0.15$ & & $<1.90$ \\
\hline $48.2-2-5,16$ & $<4.60$ & & $<0.70$ & & 55.00 & $<0.66$ & & $<0.030$ & $<0.15$ & & $<1.90$ \\
\hline $48.2-2-6,16$ & $<4.60$ & & $<0.70$ & & $>60.00$ & $<0.66$ & & $<0.030$ & $<0.15$ & & $<1.90$ \\
\hline $48.2-2-\mathrm{CC}$ & $<4.60$ & & $<0.70$ & & 57.60 & $<0.66$ & & $<0.030$ & $<0.15$ & & $<1.90$ \\
\hline $48.2-3-1,117$ & $<4.60$ & & $<0.70$ & & 51.30 & $<0.66$ & & $<0.030$ & $<0.15$ & & $<1.90$ \\
\hline $48.2-3-2,18$ & $<4.60$ & & $<0.70$ & & 34.70 & $<0.66$ & & $<0.030$ & $<0.15$ & & $<1.90$ \\
\hline $48.2-3-3,17$ & $<4.60$ & & $<0.70$ & & 47.80 & $<0.66$ & & $<0.030$ & $<0.15$ & & $<1.90$ \\
\hline $48.2-3-4,17$ & $<4.60$ & & $<0.70$ & & $>60.00$ & $<0.66$ & & $<0.030$ & $<0.15$ & & $<1.90$ \\
\hline $48.2-3-5,16$ & $<4.60$ & & $<0.70$ & & 56.20 & $<0.66$ & & $<0.030$ & $<0.15$ & & $<1.90$ \\
\hline $49.0-1-1,11-13$ & 42.30 & & 13.95 & & 2.79 & 3.09 & & $>0.210$ & 0.59 & & 5.75 \\
\hline $49.0-1-1,76$ & 29.50 & 3.31 & 8.90 & 19.78 & 21.40 & 2.16 & 9.91 & 0.050 & 0.45 & 8.84 & 3.98 \\
\hline $49.0-1-2,16-18$ & 48.50 & & 12.89 & & 6.24 & 2.29 & & $>0.210$ & 0.38 & & 4.26 \\
\hline $49.0-1-3,16$ & 52.50 & 3.32 & 15.80 & 24.31 & 1.97 & 3.80 & 0.52 & $>0.210$ & 0.65 & 8.95 & 5.82 \\
\hline $49.0-1-4,6-8$ & 49.50 & & 13.95 & & 2.02 & 3.20 & & $>0.210$ & 0.57 & & 6.16 \\
\hline $49.0-1-5,7$ & 55.60 & 3.35 & 16.60 & 25.94 & 1.95 & 3.39 & 0.58 & $>0.210$ & 0.64 & 9.17 & 5.87 \\
\hline $49.0-1-5,70-72$ & 46.30 & & 13.80 & & 1.57 & 3.20 & & $>0.210$ & 0.72 & & 6.10 \\
\hline $49.0-1$ & 83.20 & 50.12 & 1.66 & & $<0.88$ & $<0.66$ & & $<0.030$ & $<0.15$ & & $<1.90$ \\
\hline $49.0-1$ & 52.50 & 3.28 & 16.00 & 19.51 & 2.14 & 3.35 & 0.64 & $>0.210$ & 0.82 & 7.44 & 6.10 \\
\hline $49.0-2-1,70-71$ & $<4.60$ & & $<0.70$ & & 38.20 & $<0.66$ & & $<0.030$ & $<0.15$ & & $<1.90$ \\
\hline $49.1-1-1,77-79$ & 46.80 & & 14.62 & & 7.50 & 3.06 & & $>0.210$ & 0.54 & & 4.67 \\
\hline $49.1-1-2,16$ & 45.70 & 3.31 & 13.80 & 20.91 & 1.95 & 3.17 & 0.62 & $>0.210$ & 0.66 & 8.42 & 5.56 \\
\hline $49.1-1-2,124-126$ & 41.70 & & 12.60 & & 5.36 & 2.24 & & $>0.210$ & 0.40 & & 4.77 \\
\hline $49.1-1-3,22$ & 44.20 & 3.51 & 12.60 & 19.09 & 6.92 & 2.54 & 2.54 & $>0.210$ & 0.66 & 8.73 & 5.76 \\
\hline $49.1-1-4,17-19$ & 44.10 & & 13.80 & & 9.45 & 3.80 & & $>0.210$ & 0.89 & & 6.76 \\
\hline $49.1-1-4,117$ & 16.60 & 0.54 & 30.50 & 145.24 & 31.60 & 1.59 & 19.87 & $>0.210$ & 0.21 & 12.10 & 2.54 \\
\hline $49.1-1-5,38-40$ & $<4.60$ & & $<0.70$ & & $>60.00$ & $<0.66$ & & 0.040 & $<0.15$ & & $<1.90$ \\
\hline $49.1-1-5,60$ & 13.60 & 2.85 & 15.30 & 21.55 & 3.99 & 3.46 & 1.15 & $>0.210$ & 0.71 & 8.51 & 6.04 \\
\hline $49.1-2-\mathrm{CC}$ & 77.60 & 34.64 & 2.24 & & $<0.88$ & $<0.66$ & & $<0.030$ & $<0.15$ & & $<1.90$ \\
\hline $49.1-2-1,145$ & $<4.60$ & & $<0.70$ & & 48.00 & $<0.66$ & & 0.180 & $<0.15$ & & $<1.90$ \\
\hline $49.1-2-2,145-1$ & $<4.60$ & & $<0.70$ & & 69.50 & $<0.66$ & & 0.033 & $<0.15$ & & $<1.80$ \\
\hline $49.1-2-3,145$ & $<4.60$ & & $<0.70$ & & 41.30 & 0.92 & & 0.032 & 0.21 & & 1.97 \\
\hline $50.0-2-\mathrm{CC}$ & 93.40 & 35.50 & 2.63 & & $<0.88$ & $<0.66$ & & $<0.030$ & $<0.15$ & & $<1.90$ \\
\hline $50.0-2-1,145$ & 9.33 & & $<0.07$ & & 45.20 & 0.60 & 75.33 & 0.120 & $<0.15$ & & $<1.90$ \\
\hline
\end{tabular}


TABLE 7 - Continued

\begin{tabular}{|c|c|c|c|c|c|c|c|c|c|c|c|}
\hline Sample Designation & $\begin{array}{c}\mathrm{SiO}_{2} \\
(\%)\end{array}$ & $\mid \begin{array}{l}\mathrm{SiO}_{2} / \\
\mathrm{Al}_{2} \mathrm{O}_{3}\end{array}$ & $\begin{array}{c}\mathrm{A}_{2} \mathrm{O}_{3} \\
(\%)\end{array}$ & $\begin{array}{l}\mathrm{Al}_{2} \mathrm{O}_{3} / \\
\mathrm{TiO}_{2}\end{array}$ & $\begin{array}{l}\mathrm{CaO} \\
(\%)\end{array}$ & $\begin{array}{c}\mathrm{MgO} \\
(\%)\end{array}$ & $\mathrm{CaO} / \mathrm{MgO}$ & $\begin{array}{l}\mathrm{MnO} \\
(\%)\end{array}$ & $\begin{array}{l}\mathrm{TiO}_{2} \\
(\%)\end{array}$ & $\begin{array}{c}\mathrm{Fe}_{2} \mathrm{O}_{3} / \\
\mathrm{TiO}_{2}\end{array}$ & $\begin{array}{c}\mathrm{Fe}_{2} \mathrm{O}_{3} \\
(\%)\end{array}$ \\
\hline $50.0-2-2,150$ & 9.55 & & 0.70 & & 37.20 & 0.14 & & $<0.660$ & $<0.15$ & & $<1.90$ \\
\hline $50.0-2-3,150$ & 54.30 & 2.89 & 18.80 & 23.80 & 5.68 & 2.57 & 2.28 & $>0.210$ & 0.79 & 10.29 & 8.12 \\
\hline $50.0-2-4,150$ & 23.20 & & $<0.70$ & & 41.70 & $<0.66$ & & 0.150 & $<0.15$ & & $<1.90$ \\
\hline $50.0-2-6,150$ & 45.40 & & $<0.70$ & & 26.90 & $<0.14$ & & $<0.660$ & $<0.15$ & & $<1.90$ \\
\hline $50.1-1-1,62-64$ & 51.30 & & 13.95 & & 7.59 & 2.69 & & $>0.210$ & 0.54 & & 5.75 \\
\hline $50.1-1-2,15-17$ & 57.50 & & 15.50 & & 1.80 & 2.39 & & $>0.210$ & 0.60 & & 5.96 \\
\hline $50.1-1-3,7$ & 46.80 & 4.84 & 9.66 & 23.00 & 13.50 & 2.40 & 5.63 & 0.270 & 0.42 & 10.62 & 4.46 \\
\hline $50.1-1-4,17-19$ & 49.50 & & 14.45 & & 10.80 & 3.02 & & 0.195 & 0.65 & & 5.62 \\
\hline $50.1-1-5,15$ & 36.30 & 3.85 & 9.44 & 23.60 & 18.20 & 2.32 & 7.84 & 0.190 & 0.40 & 9.95 & 3.98 \\
\hline $50.1-1-6,16$ & 42.60 & 3.09 & 13.80 & 21.23 & 9.10 & 1.86 & 4.89 & $>0.210$ & 0.65 & 8.08 & 5.25 \\
\hline $50.1-2-1,130$ & 40.30 & 3.91 & 10.30 & 21.46 & 12.30 & 2.57 & 4.79 & 0.270 & 0.48 & 9.21 & 4.42 \\
\hline $50.1-2-3,4-6$ & 56.30 & & 16.00 & & 2.16 & 3.09 & & $>0.210$ & 0.71 & & 7.00 \\
\hline $50.1-2-3,35$ & 58.80 & 3.84 & 15.30 & 30.60 & 2.40 & 3.24 & 0.74 & $>0.210$ & 0.50 & 11.06 & 5.53 \\
\hline $50.1-2-4,12-14$ & 51.30 & & 15.66 & & 1.35 & 3.54 & & $>0.210$ & 0.56 & & 6.84 \\
\hline $50.1-2-4,50$ & 47.90 & 3.17 & 15.10 & 20.41 & 1.41 & 3.47 & 0.41 & $>0.210$ & 0.74 & 8.93 & 6.61 \\
\hline $50.1-2-5,8$ & 58.20 & 3.68 & 15.80 & 21.94 & 1.20 & 3.63 & 0.33 & $>0.210$ & 0.72 & 8.88 & 6.39 \\
\hline $50.1-3-1,145$ & 44.10 & 2.26 & 19.50 & 24.68 & 1.15 & 3.39 & 0.34 & $>0.210$ & 0.79 & 8.96 & 6.92 \\
\hline $50.1-3-3,15$ & 53.00 & 3.63 & 14.60 & 25.17 & 1.20 & 3.09 & 0.39 & $>0.210$ & 0.58 & 9.14 & 5.30 \\
\hline $50.1-3-4,16$ & 49.50 & & 15.10 & & 1.32 & 3.63 & & $>0.210$ & 0.73 & & 5.55 \\
\hline $50.1-3-5,28$ & 47.30 & 3.19 & 14.80 & 20.29 & 1.64 & 3.24 & 0.51 & $>0.210$ & 0.73 & 8.55 & 6.24 \\
\hline $50.1-3-6,16$ & 48.40 & & 15.30 & & 1.48 & 3.72 & & $>0.210$ & 0.53 & & 5.37 \\
\hline $50.1-4-1,75$ & 50.00 & & 16.20 & & 2.19 & 3.17 & & $>0.210$ & 0.74 & & 3.89 \\
\hline $51.0-1-\mathrm{CC}$ & 51.30 & 3.02 & 17.00 & 33.33 & 1.10 & 3.72 & 0.30 & $>0.210$ & 0.51 & 13.90 & 7.09 \\
\hline $51.0-1-1,11$ & 49.00 & 3.06 & 16.00 & 21.05 & 1.86 & 2.95 & 0.63 & $>0.210$ & 0.76 & 10.21 & 7.76 \\
\hline $51.0-1-2,8-10$ & 52.40 & 17.00 & 17.00 & & 1.10 & 3.35 & & $>0.210$ & 0.72 & & 7.40 \\
\hline $51.0-1-3,6$ & 51.30 & 3.02 & 17.00 & 27.42 & 1.12 & 3.13 & 0.36 & $>0.210$ & 0.62 & 10.06 & 6.27 \\
\hline $51.0-1-4,6-8$ & 50.70 & & 19.00 & & 1.29 & 3.42 & & $>0.210$ & 0.62 & & 7.58 \\
\hline $51.0-1-5,15$ & 45.60 & 3.02 & 15.10 & 21.88 & 1.76 & 2.62 & 0.69 & $>0.210$ & 0.69 & 10.28 & 7.09 \\
\hline $51.0-1-6,16-18$ & 48.40 & & 17.20 & & 1.55 & 3.23 & & $>0.210$ & 0.84 & & 7.67 \\
\hline $51.0-2-\mathrm{CC}$ & 46.70 & 2.94 & 15.90 & 20.13 & 1.23 & 3.47 & 0.35 & $>0.210$ & 0.79 & 10.04 & 7.93 \\
\hline $51.1-1-1,15$ & 40.70 & 3.45 & 11.80 & 15.13 & 1.48 & 2.66 & 0.56 & $>0.210$ & 0.78 & 8.90 & 7.00 \\
\hline $51.1-1-1,145$ & 61.00 & 3.63 & 16.80 & 32.94 & 1.48 & 3.31 & 0.48 & $>0.210$ & 0.51 & 9.32 & 4.78 \\
\hline $51.1-1-2,16$ & 56.30 & & 15.10 & & 2.48 & 3.05 & & 0.150 & 0.60 & & 5.76 \\
\hline $51.1-1-3,30$ & 65.30 & 4.27 & 15.30 & 27.32 & 2.04 & 2.79 & 0.73 & 0.180 & 0.56 & 10.75 & 6.02 \\
\hline $51.1-1-4,16$ & 57.50 & & 15.80 & & 1.40 & 3.31 & & $>0.210$ & 0.68 & & 5.63 \\
\hline $51.1-1 \cdot 4,30$ & 53.60 & 3.12 & 17.20 & 28.69 & 1.41 & 3.02 & 0.49 & 0.190 & 0.60 & 10.28 & 6.17 \\
\hline $51.1-1-5,16$ & 59.60 & 3.90 & 15.30 & 31.88 & 2.40 & 1.97 & 1.22 & 0.110 & 0.48 & 9.88 & 4.74 \\
\hline $51.1-1-6,16$ & 58.20 & & 15.50 & & 1.12 & 3.24 & & 0.210 & 0.56 & & 5.37 \\
\hline $51.1-2-1,127$ & 42.60 & 3.09 & 13.80 & 19.40 & 1.45 & 2.75 & 0.53 & $>0.210$ & 0.81 & 8.54 & 6.92 \\
\hline $51.1-2-2,8$ & 50.00 & & 17.00 & & 1.10 & 3.17 & & $>0.210$ & 0.82 & & 6.17 \\
\hline $52.0-1-1,27$ & 51.30 & 3.31 & 15.50 & 22.80 & 1.82 & 3.39 & 0.54 & $>0.210$ & 0.68 & 9.41 & 6.60 \\
\hline $52.0-1-2,8$ & 57.50 & & 16.20 & & 1.74 & 3.20 & & $>0.210$ & 0.66 & & 5.89 \\
\hline $52.0-1-2,30$ & 50.60 & 3.26 & 15.50 & 24.60 & 1.51 & 3.35 & 0.45 & $>0.210$ & 0.63 & 10.34 & 6.53 \\
\hline $52.0-1-3,8$ & 55.60 & 3.81 & 14.60 & 26.55 & 2.22 & 3.02 & 0.74 & $>0.210$ & 0.55 & 10.00 & 5.55 \\
\hline $52.0-1-4,8$ & 55.00 & & 15.80 & & 1.41 & 3.43 & & $>0.210$ & 0.78 & & 6.31 \\
\hline $52.0-1-5,10$ & 43.70 & 3.01 & 14.50 & 18.13 & 2.13 & 3.31 & 0.65 & $>0.210$ & 0.80 & 8.65 & 6.92 \\
\hline $52.0-1-6,9$ & 49.50 & & 1.62 & & 1.40 & 3.59 & & $>0.210$ & 0.74 & & 6.40 \\
\hline $52.0-2-\mathrm{CC}$ & 49.50 & 24.90 & 19.90 & 26.18 & 1.62 & 3.27 & 0.50 & $>0.210$ & 0.76 & 10.22 & 7.77 \\
\hline
\end{tabular}


TABLE 7 - Continued

\begin{tabular}{|c|c|c|c|c|c|c|c|c|c|c|c|}
\hline Sample Designation & $\begin{array}{c}\mathrm{SiO}_{2} \\
(\%)\end{array}$ & $\begin{array}{l}\mathrm{SiO}_{2} / \\
\mathrm{Al}_{2} \mathrm{O}_{3}\end{array}$ & $\begin{array}{c}\mathrm{Al}_{2} \mathrm{O}_{3} \\
(\%)\end{array}$ & $\begin{array}{c}\mathrm{Al}_{2} \mathrm{O}_{3} / \\
\mathrm{TiO}_{2}\end{array}$ & $\begin{array}{r}\mathrm{CaO} \\
(\%)\end{array}$ & $\begin{array}{l}\text { Mgo } \\
(\%)\end{array}$ & $\mathrm{CaO} / \mathrm{MgO}$ & $\begin{array}{c}\mathrm{MnO} \\
(\%)\end{array}$ & $\begin{array}{r}\mathrm{TiO}_{2} \\
(\%)\end{array}$ & $\begin{array}{c}\mathrm{Fe}_{2} \mathrm{O}_{3} / \\
\mathrm{TiO}_{2}\end{array}$ & $\begin{array}{c}\mathrm{Fe}_{2} \mathrm{O}_{3} \\
(\%)\end{array}$ \\
\hline $52.0-2-2,7$ & 57.60 & 3.79 & 15.20 & 27.14 & 2.14 & 3.13 & 0.68 & $>0.210$ & 0.56 & 10.64 & 5.96 \\
\hline $52.0-2-3,7$ & 56.30 & & 17.00 & & 1.70 & 3.79 & & $>0.210$ & 1.02 & & 7.67 \\
\hline $52.0-2-4,8$ & 46.70 & 3.22 & 14.50 & 22.31 & 2.51 & 3.35 & 0.75 & $>0.210$ & 0.65 & 10.89 & 7.09 \\
\hline $52.0-2-5,18$ & 49.00 & & 16.20 & & 1.70 & 3.55 & & $>0.210$ & 0.79 & & 6.31 \\
\hline $52.0-2-6,145$ & 51.80 & 2.43 & 19.00 & 25.00 & 1.45 & 3.47 & 0.42 & $>0.210$ & 0.76 & 9.53 & 7.24 \\
\hline $52.0-3-1,16$ & 56.30 & & 19.10 & & 1.41 & 4.13 & & $>0.210$ & 0.85 & & 7.25 \\
\hline $52.0-3-2,19$ & 45.60 & 3.21 & 14.20 & 20.88 & 2.09 & 3.02 & 0.69 & $>0.210$ & 0.68 & 9.29 & 6.32 \\
\hline $52.0-3-3,16$ & 56.30 & & 15.90 & & 1.00 & 4.67 & & $>0.210$ & 0.55 & & 5.37 \\
\hline $52.0-3-4,16$ & 56.30 & 3.06 & 18.40 & 36.08 & 1.35 & 4.13 & 0.33 & $>0.210$ & 0.51 & 11.50 & 5.88 \\
\hline $52.0-3-5,7$ & 51.30 & & 16.60 & & 1.62 & 3.55 & & $>0.210$ & 0.80 & & 6.60 \\
\hline $52.0-3-6,6$ & 53.60 & 2.82 & 19.00 & 25.00 & 1.88 & 3.84 & 0.49 & $>0.210$ & 0.76 & 8.40 & 6.61 \\
\hline $52.0-4-2,7$ & 56.30 & & 18.60 & & 1.48 & 4.26 & & $>0.210$ & 0.76 & & 7.07 \\
\hline $52.0-4-3,5$ & 55.00 & 3.59 & 15.30 & 30.60 & 2.29 & 3.20 & 0.72 & $>0.210$ & 0.50 & 11.50 & 5.75 \\
\hline $52.0-4-4,6$ & 51.30 & & 16.40 & & 1.82 & 4.17 & & $>0.210$ & 0.62 & & 6.17 \\
\hline $52.0-4-5,6$ & 53.20 & 3.28 & 16.20 & 23.48 & 1.48 & 3.67 & 0.40 & 0.157 & 0.69 & 12.06 & 8.32 \\
\hline $52.0-4-6,18$ & 52.40 & & 16.60 & & 1.78 & 3.80 & & $>0.210$ & 0.54 & & 6.13 \\
\hline $52.0-4-6,128$ & 53.80 & & 17.00 & & 1.40 & 3.84 & & $>0.210$ & 0.76 & & 8.40 \\
\hline $52.0-5-1,127$ & 47.80 & & 18.60 & & 1.12 & 3.63 & & $>0.210$ & 0.82 & & 6.76 \\
\hline $52.0-5-2,10$ & 52.50 & 3.32 & 15.80 & 35.11 & 2.48 & 3.47 & 0.71 & $>0.210$ & 0.45 & 14.67 & 6.60 \\
\hline $52.0-5-2,17$ & 50.60 & & 17.60 & & 1.32 & 3.55 & & $>0.210$ & 1.00 & & 7.67 \\
\hline $52.0-5-3,15$ & 49.00 & 2.63 & 18.60 & 22.41 & 1.86 & 3.55 & 0.52 & 0.210 & 0.83 & 10.02 & 8.32 \\
\hline $52.0-5-3,18$ & 51.30 & & 17.80 & & 1.51 & 3.89 & & $>0.210$ & 0.70 & & 6.76 \\
\hline $52.0-5-4,18$ & 41.70 & 2.51 & 16.60 & 20.00 & 1.52 & 3.72 & 0.41 & $>0.210$ & 0.83 & 9.57 & 7.94 \\
\hline $52.0-5-5,18$ & 46.70 & & 17.80 & & 1.12 & 3.63 & & $>0.210$ & 0.93 & & 7.25 \\
\hline $52.0-6-\mathrm{CC}$ & 51.90 & 2.92 & 17.80 & 28.25 & 1.02 & 3.63 & 0.28 & $>0.210$ & 0.63 & 12.62 & 7.95 \\
\hline $52.0-6-1,24$ & 55.60 & 2.85 & 19.50 & 22.41 & 1.35 & 3.55 & 0.42 & $>0.210$ & 0.87 & 9.11 & 7.93 \\
\hline $52.0-7-2,37-39$ & 45.70 & & 16.00 & & 2.16 & 2.95 & & $>0.210$ & 0.48 & & 6.52 \\
\hline $52.0-7-\mathrm{CC}$ & 79.40 & 35.13 & 2.26 & & 1.26 & 1.00 & 1.26 & $>0.210$ & $<0.15$ & & $<1.90$ \\
\hline $52.0-8-1,145$ & 63.00 & 6.53 & 9.65 & 30.16 & 2.14 & 2.92 & 0.73 & $>0.210$ & 0.32 & 12.75 & 4.08 \\
\hline $52.0-8-2,145-150$ & 54.30 & & 10.12 & & 3.22 & 2.75 & & $>0.210$ & 0.52 & & 5.19 \\
\hline $52.0-8-3,21$ & 53.70 & 5.26 & 10.20 & 21.25 & 2.57 & 2.82 & 0.31 & $>0.210$ & 0.48 & 10.46 & 5.02 \\
\hline $52.0-8-3,40$ & 67.60 & 9.14 & 7.40 & 29.60 & 1.58 & 2.40 & 0.13 & $>0.210$ & 0.25 & 13.56 & 3.39 \\
\hline $52.0-8-5,145-150$ & 65.10 & & 5.70 & & 1.27 & 1.70 & & $>0.210$ & 0.32 & & 3.55 \\
\hline $52.0-8-6,145$ & 44.60 & 3.16 & 14.10 & 21.69 & 3.31 & 3.47 & 0.95 & $>0.210$ & 0.65 & 8.85 & 5.70 \\
\hline $52.0-9-\mathrm{CC}$ & 53.10 & 3.28 & 16.20 & 32.40 & 1.82 & 3.20 & 0.57 & $>0.210$ & 0.50 & 11.36 & 5.68 \\
\hline $52.0-10-\mathrm{CC}$ & 45.70 & 2.69 & 17.00 & 31.48 & 1.86 & 3.55 & 0.52 & $>0.210$ & 0.54 & 12.22 & 6.60 \\
\hline $53.0-1-1,10-12$ & 51.30 & & 13.80 & & 5.50 & 3.98 & & 0.158 & 0.74 & & 8.23 \\
\hline $53.0-1-2,144$ & 52.50 & 4.04 & 13.00 & 22.03 & 4.46 & 2.46 & 1.81 & 0.130 & 0.59 & 10.08 & 5.95 \\
\hline $53.0-1-3,17-19$ & 55.00 & & 13.95 & & 4.37 & 2.48 & & 0.128 & 0.49 & & 6.16 \\
\hline $53.0-2-\mathrm{CC}$ & 50.60 & & 13.80 & & 4.85 & 3.88 & & 0.170 & 0.87 & & 8.70 \\
\hline $53.0-2-\mathrm{CC}$ & 63.10 & & 13.80 & & 4.46 & 3.35 & & 0.143 & 0.49 & & 5.19 \\
\hline $53.0-3-1,17$ & 46.80 & 33.91 & 1.38 & 1.92 & 4.16 & 0.14 & 0.98 & 4.260 & 0.72 & 10.31 & 7.42 \\
\hline $53.0-4-2,0$ & 51.30 & 4.89 & 10.50 & & 1.50 & 4.78 & 0.31 & 0.120 & 0.61 & & 9.00 \\
\hline $53.0-6-2,65$ & 5.90 & 2.95 & 2.00 & & 45.60 & 1.12 & 40.71 & $>0.210$ & $<0.15$ & & 2.34 \\
\hline $53.0-6-2,66-67$ & 38.90 & & $<0.70$ & & 46.70 & 1.20 & & 0.176 & $<0.15$ & & 2.63 \\
\hline $53.0-6-\mathrm{CC}$ & 15.30 & 5.43 & 2.82 & 16.59 & 35.10 & 4.46 & 7.87 & 0.210 & 0.17 & 12.29 & 2.09 \\
\hline $53.0-7-1,150$ & 38.40 & & 15.12 & & 18.00 & 3.94 & & 0.100 & 0.94 & & 2.48 \\
\hline $53.0-8-\mathrm{CC}$ & 51.30 & 3.09 & 16.60 & 150.91 & 5.00 & 6.68 & 0.75 & 0.070 & 0.11 & 82.73 & 9.10 \\
\hline
\end{tabular}


TABLE 7 - Continued

\begin{tabular}{|c|c|c|c|c|c|c|c|c|c|c|c|}
\hline Sample Designation & $\begin{array}{c}\mathrm{SiO}_{2} \\
(\%)\end{array}$ & $\begin{array}{l}\mathrm{SiO}_{2} / \\
\mathrm{Al}_{2} \mathrm{O}_{3}\end{array}$ & $\begin{array}{c}\mathrm{Al}_{2} \mathrm{O}_{3} \\
(\%)\end{array}$ & $\begin{array}{c}\mathrm{Al}_{2} \mathrm{O}_{3} / \\
\mathrm{TiO}_{2}\end{array}$ & $\begin{array}{l}\mathrm{CaO} \\
(\%)\end{array}$ & $\begin{array}{l}\mathrm{MgO} \\
(\%)\end{array}$ & $\mathrm{CaO} / \mathrm{MgO}$ & $\begin{array}{c}\mathrm{MnO} \\
(\%)\end{array}$ & $\begin{array}{l}\mathrm{TiO}_{2} \\
(\%)\end{array}$ & $\begin{array}{c}\mathrm{Fe}_{2} \mathrm{O}_{3} / \\
\mathrm{SiO}_{2}\end{array}$ & $\begin{array}{c}\mathrm{Fe}_{2} \mathrm{O}_{3} \\
(\%)\end{array}$ \\
\hline $53.1-1-1,7-9$ & 44.30 & & 14.30 & & 3.85 & 3.02 & & $>0.210$ & 0.90 & & $>10.00$ \\
\hline $53.1-1-2,7$ & 42.60 & 2.51 & 17.00 & 19.54 & 2.70 & 3.24 & 0.83 & $>0.210$ & 0.87 & & $>10.00$ \\
\hline $53.1-1-2,70-72$ & 39.50 & & 13.80 & & 3.67 & 2.98 & & $>0.210$ & 0.91 & & $>10.00$ \\
\hline $53.1-1-3,18$ & 43.60 & 2.99 & 14.60 & 16.40 & 3.90 & 2.95 & 1.32 & $>0.210$ & 0.89 & 6.18 & 5.50 \\
\hline $53.1-1-4,17-19$ & 41.20 & & 13.65 & & 4.84 & 2.69 & & $>0.210$ & 0.92 & & $>10.00$ \\
\hline $53.1-1-5,18$ & 44.60 & 2.88 & 15.50 & 24.22 & 3.55 & 3.02 & 1.18 & $>0.210$ & 0.64 & 13.91 & 8.90 \\
\hline $53.1-1-6,28-30$ & 46.80 & & 15.15 & & 2.57 & 3.23 & & $>0.210$ & 0.89 & & $>10.00$ \\
\hline $53.1-2-1,145$ & 57.50 & 3.30 & 17.40 & 31.07 & 5.26 & 2.12 & 2.48 & 0.230 & 0.56 & 10.89 & 6.10 \\
\hline $53.1-2-2,145$ & 54.40 & 3.51 & 15.50 & 39.74 & 6.30 & 2.19 & 2.88 & 0.210 & 0.39 & 12.17 & 5.02 \\
\hline $53.1-2-3,16-18$ & 61.00 & & 14.62 & & 5.50 & 2.09 & & $>0.210$ & 0.52 & & 5.62 \\
\hline $53.1-2-3,90$ & 50.20 & 3.98 & 12.60 & 16.15 & 5.76 & 2.60 & 2.22 & 0.200 & 0.78 & 9.62 & 7.50 \\
\hline $53.1-2-4,10-12$ & 50.70 & & 15.15 & & 6.46 & 2.66 & & $>0.210$ & 0.72 & & 7.24 \\
\hline $53.1-2-5,7$ & 55.60 & 3.52 & 15.80 & 17.36 & 6.03 & 2.40 & 2.51 & $>0.210$ & 0.91 & & $>10.00$ \\
\hline $53.1-2-6,7-9$ & 44.90 & & 14.48 & & 5.37 & 2.57 & & 0.186 & 0.76 & & 7.07 \\
\hline $53.1-3-1,150$ & 47.90 & 2.81 & 17.00 & 21.25 & 8.22 & 3.20 & 2.56 & $>0.210$ & 0.80 & 12.50 & 10.00 \\
\hline $53.1-3-2,16$ & 43.60 & 3.49 & 12.50 & 14.04 & 5.90 & 2.42 & 2.44 & $>0.210$ & 0.89 & 6.63 & 5.90 \\
\hline $53.1-3-2,40$ & 53.00 & 2.95 & 18.00 & 27.69 & 5.50 & 2.95 & 1.86 & $>0.210$ & 0.65 & 12.49 & 8.12 \\
\hline $53.1-3-3,23-25$ & 50.00 & & 12.60 & & 7.76 & 2.43 & & 0.166 & 0.63 & & 7.50 \\
\hline $53.1-3-4,26$ & 56.30 & 3.73 & 15.10 & 16.24 & 6.39 & 2.82 & 2.27 & 0.170 & 0.93 & 10.39 & 9.66 \\
\hline $53.1-3-5,23-25$ & 48.40 & & 12.45 & & 8.32 & 2.82 & & 0.182 & 0.88 & & 8.14 \\
\hline $53.2-1-1,21-22$ & 45.70 & & 15.32 & & 3.43 & 3.72 & & $>0.210$ & 0.94 & & $>10.00$ \\
\hline $53.2-1-2,16$ & 49.00 & 3.71 & 13.20 & 19.70 & 7.78 & 2.14 & 3.64 & $>0.210$ & 0.67 & 14.25 & 9.55 \\
\hline $53.2-1-3,22-24$ & 45.70 & & 14.80 & & 9.45 & 2.95 & & $>0.210$ & 0.85 & & 9.10 \\
\hline $53.2-1-4,7$ & 42.70 & 3.09 & 13.80 & 17.04 & 8.91 & 2.45 & 3.64 & 0.186 & 0.81 & 11.23 & 9.10 \\
\hline $53.2-1-5,7-9$ & 53.20 & & 15.00 & & 8.33 & 2.37 & & $>0.210$ & 0.73 & & 8.11 \\
\hline $53.2-1-6,7$ & 50.20 & & 17.40 & & 6.17 & 2.26 & & 0.250 & 0.69 & & 7.86 \\
\hline $53.2-4-4, \mathrm{CC}$ & 50.00 & & 12.89 & & 3.71 & 1.52 & & $>0.210$ & 7.16 & & $>_{10.00}$ \\
\hline $53.2-5-5, \mathrm{CC}$ & 57.50 & & 11.32 & & 2.16 & 4.07 & & $>0.210$ & 1.00 & & 7.00 \\
\hline $54.0-1-1,5$ & 9.10 & & $<0.70$ & & 41.20 & $<0.66$ & & 0.130 & $<0.15$ & & $<1.90$ \\
\hline $54.0-1-1,74-76$ & 55.00 & & 14.95 & & 6.46 & 2.11 & & 0.151 & 0.52 & & 6.60 \\
\hline $54.01-2,7$ & 53.80 & 3.96 & 13.60 & 28.86 & 7.95 & 2.46 & 3.23 & 0.162 & 0.35 & 16.42 & 5.75 \\
\hline $54.0-1-2,20$ & 53.00 & 3.84 & 13.80 & 21.56 & 7.50 & 2.69 & 2.79 & 0.180 & 0.64 & 9.97 & 6.38 \\
\hline $54.0-1-2,20$ & 47.80 & 4.02 & 11.90 & 19.83 & 56.30 & 2.26 & 24.91 & 0.170 & 0.60 & 9.60 & 5.76 \\
\hline $54.0-1-3,35-37$ & 61.00 & & 14.62 & & 7.25 & 2.88 & & 0.148 & 0.55 & & 6.85 \\
\hline $54.0-2-1,17$ & 55.60 & 4.03 & 13.80 & 26.54 & 7.10 & 1.82 & 3.90 & 0.150 & 0.52 & 10.81 & 5.62 \\
\hline $54.0-2-2,23-25$ & 59.50 & & 12.30 & & 10.20 & 2.51 & & 0.141 & 0.46 & & 6.24 \\
\hline $54.0-2-4,16$ & 49.00 & 3.89 & 12.60 & 24.23 & 8.92 & 1.74 & 5.13 & 0.133 & 0.52 & 10.58 & 5.50 \\
\hline $54.0-3-1,140$ & 48.40 & 3.67 & 13.20 & 24.44 & 11.40 & 1.45 & 7.86 & 0.140 & 0.54 & 7.37 & 3.98 \\
\hline $54.0-4-1,108-110$ & 52.40 & & 11.88 & & 8.20 & 1.00 & & 0.130 & 0.50 & & 4.73 \\
\hline $54.0-4-2,14$ & 55.60 & 4.41 & 12.62 & 27.39 & 9.76 & 1.55 & 6.30 & 0.170 & 0.46 & 10.65 & 4.90 \\
\hline $54.0-6-1,145$ & 51.20 & 3.56 & 14.40 & 22.86 & 4.95 & 1.26 & 3.93 & 0.150 & 0.63 & 7.78 & 4.90 \\
\hline $54.0-6-2,16-18$ & 52.40 & & 13.50 & & 4.85 & 1.33 & & 0.133 & 0.55 & & 5.50 \\
\hline $54.0-6-3,23$ & 49.00 & 4.30 & 11.40 & 25.33 & 10.00 & 1.95 & 5.13 & 0.13 & 0.45 & 11.27 & 5.07 \\
\hline $54.0-6-1,16-18$ & 62.40 & & 15.12 & & 5.50 & 2.75 & & 0.151 & 0.46 & & 5.88 \\
\hline $54.0-6-4,78$ & 52.50 & 4.57 & 11.50 & 24.47 & 8.72 & 1.70 & 5.13 & 0.132 & 0.47 & 10.69 & 5.00 \\
\hline $54.0-6-5,17-19$ & 50.70 & & 12.60 & & 5.95 & 7.86 & & 0.138 & 0.47 & & 5.24 \\
\hline $54.0-7-2,34-36$ & 58.80 & & 13.18 & & 7.37 & 1.51 & & 0.210 & 0.51 & & 5.62 \\
\hline $54.0-7-1,138$ & 55.60 & 9.31 & 12.90 & 26.88 & 5.25 & 1.70 & 3.09 & $>0.210$ & 0.48 & 9.10 & 4.37 \\
\hline
\end{tabular}


TABLE 7 - Continued

\begin{tabular}{|c|c|c|c|c|c|c|c|c|c|c|c|}
\hline Sample Designation & $\begin{array}{c}\mathrm{SiO}_{2} \\
(\%)\end{array}$ & $\begin{array}{l}\mathrm{SiO}_{2} / \\
\mathrm{Al}_{2} \mathrm{O}_{3}\end{array}$ & $\begin{array}{c}\mathrm{Al}_{2} \mathrm{O}_{3} \\
(\%)\end{array}$ & $\begin{array}{c}\mathrm{Al}_{2} \mathrm{O}_{3} / \\
\mathrm{TiO}_{2}\end{array}$ & $\begin{array}{r}\mathrm{CaO} \\
(\%)\end{array}$ & $\begin{array}{l}\mathrm{MgO} \\
(\%)\end{array}$ & $\mathrm{CaO} / \mathrm{MgO}$ & $\begin{array}{l}\mathrm{MnO} \\
(\%)\end{array}$ & $\begin{array}{c}\mathrm{TiO}_{2} \\
(\%)\end{array}$ & $\begin{array}{c}\mathrm{Fe}_{2} \mathrm{O}_{3} / \\
\mathrm{TiO}_{2}\end{array}$ & $\begin{array}{c}\mathrm{Fe}_{2} \mathrm{O}_{3} \\
(\%)\end{array}$ \\
\hline $54.0-7-3,16$ & 56.20 & 4.68 & 12.00 & 21.82 & 3.98 & 1.20 & 3.32 & 0.190 & 0.55 & 9.55 & 5.25 \\
\hline $54.0-9-1$ & 18.60 & 2.37 & 7.83 & 16.70 & 30.90 & 3.55 & 8.70 & 0.102 & 0.47 & 10.09 & 4.74 \\
\hline $55.0-1-1,7-9$ & $<4.60$ & & $<0.70$ & & 56.20 & $<0.66$ & & 0.063 & $<0.15$ & & $<1.80$ \\
\hline $55.0-1-2,7$ & $<4.60$ & & $<0.70$ & & 43.70 & $<0.66$ & & 0.107 & $<0.15$ & & $<1.90$ \\
\hline $55.0-1-2,30$ & $<4.60$ & & $<0.70$ & & 44.60 & $<0.66$ & & 0.110 & $<0.15$ & & $<1.90$ \\
\hline $55.0-1-3,7-9$ & $<4.60$ & & $<0.70$ & & 49.00 & $<0.66$ & & 0.098 & $<0.15$ & & $<1.90$ \\
\hline $55.0-1-4,7$ & $<4.60$ & & $<0.70$ & & 46.80 & $<0.66$ & & 0.091 & $<0.15$ & & $<1.90$ \\
\hline $55.0-1-5.7-9$ & $<4.60$ & & $<0.70$ & & 50.70 & $<0.66$ & & 0.055 & $<0.15$ & & $<1.90$ \\
\hline $55.0-1-6,7$ & $<4.60$ & & $<0.70$ & & 49.00 & $<0.66$ & & 0.065 & $<0.15$ & & $<1.90$ \\
\hline $55.0-2-1,7-9$ & $<4.60$ & & $<0.70$ & & 45.80 & $<0.66$ & & 0.072 & $<0.15$ & & $<1.90$ \\
\hline $55.0-2-2,7$ & $<4.60$ & & $<0.70$ & & 46.80 & $<0.66$ & & $<0.030$ & $<0.15$ & & $<1.90$ \\
\hline $55.0-2-4,15$ & $<4.60$ & & $<0.70$ & & 50.00 & $<0.66$ & & 0.035 & $<0.15$ & & $<1.90$ \\
\hline $55.0-2-5,7-9$ & $<4.60$ & & $<0.70$ & & 52.40 & $<0.66$ & & 0.032 & $<0.15$ & & $<1.90$ \\
\hline $55.0-2-5,70$, & $<4.60$ & & $<0.70$ & & 46.80 & $<0.66$ & & $<0.030$ & $<0.15$ & & $<1.90$ \\
\hline $55.0-2-6,32-37$ & $<4.60$ & & $<0.70$ & & 45.80 & $<0.66$ & & $<0.030$ & $<0.15$ & & $<1.90$ \\
\hline $55.0-3-1,16$ & $<4.60$ & & $<0.70$ & & 46.80 & $<0.66$ & & $<0.030$ & $<0.15$ & & $<1.90$ \\
\hline $55.0-3-3,14-16$ & $<4.60$ & & $<0.70$ & & 47.90 & $<0.66$ & & 0.030 & $<0.15$ & & $<1.90$ \\
\hline $55.0-3-4,145$ & $<4.60$ & & $<0.70$ & & 54.40 & $<0.66$ & & $<0.030$ & $<0.15$ & & $<1.90$ \\
\hline $55.0-3-5,15-17$ & $<4.60$ & & $<0.70$ & & 44.60 & $<0.66$ & & $<0.030$ & $<0.15$ & & $<1.90$ \\
\hline $55.0-4-1,13$ & $<4.60$ & & $<0.70$ & & 46.80 & $<0.66$ & & $<0.030$ & $<0.15$ & & $<1.90$ \\
\hline $55.0-4-2,145-150$ & $<4.60$ & & $<0.70$ & & 47.90 & $<0.66$ & & $<0.030$ & $<0.15$ & & $<1.90$ \\
\hline $55.0-4-3,7$ & $<4.60$ & & $<0.70$ & & 50.00 & $<0.66$ & & $<0.030$ & $<0.15$ & & $<1.90$ \\
\hline $55.0-4-4,145-150$ & $<4.60$ & & $<0.70$ & & 56.20 & $<0.66$ & & $<0.030$ & $<0.15$ & & $<1.90$ \\
\hline $55.0-4-5,7$ & $<4.60$ & & $<0.70$ & & 40.70 & $<0.66$ & & $<0.030$ & $<0.15$ & & $<1.90$ \\
\hline $55.0-4-6,145-150$ & $<4.60$ & & $<0.70$ & & 53.10 & $<0.66$ & & $<0.030$ & $<0.15$ & & $<1.90$ \\
\hline $55.0-5-1,7$ & $<4.60$ & & $<0.70$ & & 47.80 & $<0.66$ & & $<0.030$ & $<0.15$ & & $<1.90$ \\
\hline $55.0-5-3,10$ & $<4.60$ & & $<0.70$ & & 53.70 & $<0.66$ & & $<0.030$ & $<0.15$ & & $<1.90$ \\
\hline $55.0-5-5,7$ & $<4.60$ & & $<0.70$ & & 51.30 & $<0.66$ & & $<0.030$ & $<0.15$ & & $<1.90$ \\
\hline $55.0-6-1,7-9$ & $<4.60$ & & $<0.70$ & & 52.40 & 0.82 & & 0.053 & $<0.15$ & & $<1.90$ \\
\hline $55.0-6-3,17$ & $<4.60$ & & $<0.70$ & & 45.70 & $<0.66$ & & 0.031 & $<0.15$ & & $<1.90$ \\
\hline $55.0-6-5,16-18$ & $<4.60$ & & $<0.70$ & & 56.20 & 0.76 & & 0.017 & $<0.15$ & & $<1.90$ \\
\hline $55.0-7-1,14$ & $<4.60$ & & $<0.70$ & & 45.70 & $<0.66$ & & $<0.030$ & $<0.15$ & & $<1.90$ \\
\hline $55.0-7-3,18-20$ & $<4.60$ & & $<0.70$ & & 52.40 & 0.68 & & 0.040 & $<0.15$ & & $<1.90$ \\
\hline $55.0-7-5,19$ & $<4.60$ & & $<0.70$ & & 50.00 & $<0.66$ & & $<0.030$ & $<0.15$ & & $<1.90$ \\
\hline $55.0-8-3,16$ & $<4.60$ & & $<0.70$ & & 36.30 & $<0.66$ & & $<0.030$ & $<0.15$ & & $<1.90$ \\
\hline $55.0-8-5,18-20$ & $<4.60$ & & $<0.70$ & & 52.40 & 0.68 & & $<0.036$ & $<0.15$ & & $<1.90$ \\
\hline $55.0-9$ & $<4.60$ & & $<0.70$ & & 49.00 & $<0.66$ & & 0.038 & $<0.15$ & & $<1.90$ \\
\hline $55.0-10-2,10$ & 9.11 & & $<0.70$ & & 44.20 & $<0.66$ & & 0.049 & $<0.15$ & & $<1.90$ \\
\hline $55.0-10-3,37-39$ & $<4.60$ & & $<0.70$ & & 52.10 & 0.74 & & 0.050 & $<0.15$ & & $<1.90$ \\
\hline $55.0-10-5,16$ & $<4.60$ & & $<0.70$ & & 45.70 & $<0.66$ & & $<0.031$ & $<0.15$ & & $<1.90$ \\
\hline $55.0-11-1,46-48$ & $<4.60$ & & $<0.70$ & & 52.40 & 0.80 & & 0.039 & $<0.15$ & & $<1.90$ \\
\hline $55.0-11-3,17$ & $<4.60$ & & $<0.70$ & & 33.90 & $<0.66$ & & 0.038 & $<0.15$ & & $<1.90$ \\
\hline $55.0-11-5,7-9$ & $<4.60$ & & $<0.70$ & & 46.70 & 0.70 & & 0.034 & $<0.15$ & & $<1.90$ \\
\hline $55.0-12-1,13$ & $<4.60$ & & $<0.70$ & & 50.00 & $<0.66$ & & 0.056 & $<0.15$ & & $<1.90$ \\
\hline $55.0-12-3,8$ & $<4.60$ & & $<0.70$ & & 45.70 & $<0.66$ & & 0.072 & $<0.15$ & & $<1.90$ \\
\hline $55.0-13-1,7$ & $<4.60$ & & $<0.70$ & & 47.80 & $<0.66$ & & 0.072 & $<0.15$ & & $<1.90$ \\
\hline $55.0-13-3,40$ & $<4.60$ & & $<0.70$ & & 44.70 & $<0.66$ & & $<0.030$ & $<0.15$ & & $<1.90$ \\
\hline $55.0-13-5,7$ & $<4.60$ & & $<0.70$ & & 45.70 & $<0.66$ & & $<0.030$ & $<0.15$ & & $<1.90$ \\
\hline
\end{tabular}


TABLE 7 - Continued

\begin{tabular}{|c|c|c|c|c|c|c|c|c|c|c|c|}
\hline Sample Designation & $\begin{array}{c}\mathrm{SiO}_{2} \\
(\%)\end{array}$ & $\begin{array}{l}\mathrm{SiO}_{2} / \\
\mathrm{Al}_{2} \mathrm{O}_{3}\end{array}$ & $\begin{array}{c}\mathrm{Al}_{2} \mathrm{O}_{3} \\
(\%)\end{array}$ & $\begin{array}{c}\mathrm{Al}_{2} \mathrm{O}_{3} / \\
\mathrm{TiO}_{2}\end{array}$ & $\begin{array}{r}\mathrm{CaO} \\
(\%)\end{array}$ & $\begin{array}{l}\mathrm{MgO} \\
(\%)\end{array}$ & $\mathrm{CaO} / \mathrm{MgO}$ & $\begin{array}{c}\mathrm{MnO} \\
(\%)\end{array}$ & $\begin{array}{c}\mathrm{TiO}_{2} \\
(\%)\end{array}$ & $\begin{array}{c}\mathrm{Fe}_{2} \mathrm{O}_{3} / \\
\mathrm{TiO}_{2}\end{array}$ & $\begin{array}{c}\mathrm{Fe}_{2} \mathrm{O}_{3} \\
(\%)\end{array}$ \\
\hline $55.0-14-2,145$ & $<4.60$ & & $<0.70$ & & 50.00 & $<0.66$ & & 0.062 & $<0.15$ & & $<1.90$ \\
\hline $55.0-14-4,145$ & $<4.60$ & & $<0.70$ & & 36.80 & $<0.66$ & & $<0.030$ & $<0.15$ & & $<1.90$ \\
\hline $55.0-14-6,145$ & $<4.60$ & & $<0.70$ & & 47.80 & $<0.66$ & & $<0.030$ & $<0.15$ & & $<1.90$ \\
\hline $56.2-3-\mathrm{CC}$ & 16.70 & & 13.95 & & 1.13 & 5.00 & & $>0.210$ & 1.48 & & $>10.00$ \\
\hline 56.2-3, Top of Core & 53.60 & & 11.49 & & 3.58 & 4.90 & & $>0.210$ & 1.82 & & $>10.00$ \\
\hline $56.2-1-2,11-13$ & 13.50 & & 1.09 & & 40.70 & 2.95 & & 0.051 & 0.37 & & 2.54 \\
\hline $56.2-1-1,23-29$ & $<4.60$ & & $<0.70$ & & 51.80 & $<0.66$ & & $<0.030$ & $<0.15$ & & $<1.90$ \\
\hline $56.2-1-6,7$ & $<4.60$ & & $<0.70$ & & 51.40 & $<0.66$ & & $<0.030$ & $<0.15$ & & $<1.90$ \\
\hline $56.2-2-4,36$ & $<4.60$ & & $<0.70$ & & 49.00 & $<0.66$ & & $<0.030$ & $<0.15$ & & $<1.90$ \\
\hline $56.2-2-6,16-18$ & $<4.60$ & & $<0.70$ & & 47.30 & $<0.66$ & & $<0.030$ & $<0.15$ & & $<1.90$ \\
\hline $56.2-2-9$, Тор 90 & $<4.60$ & & $<0.70$ & & 53.60 & $<0.66$ & & $<0.030$ & $<0.15$ & & $<1.90$ \\
\hline $56.2-3-2,145$ & $<4.60$ & & $<0.70$ & & 45.60 & $<0.66$ & & $<0.030$ & $<0.15$ & & $<1.90$ \\
\hline $56.2-4-2,10-11$ & $<4.60$ & & $<0.70$ & & 42.60 & $<0.66$ & & $<0.030$ & $<0.15$ & & $<1.90$ \\
\hline $56.2-4-4,14$ & $<4.60$ & & $<0.70$ & & 44.60 & $<0.66$ & & $<0.030$ & $<0.15$ & & $<1.90$ \\
\hline $56.2-4-5,13-15$ & $<4.60$ & & $<0.70$ & & 49.00 & $<0.66$ & & $<0.030$ & $<0.15$ & & $<1.90$ \\
\hline $56.2-5-2,13$ & $<4.60$ & & $<0.70$ & & 52.50 & $<0.66$ & & $<0.030$ & $<0.15$ & & $<1.90$ \\
\hline $56.2-5-4,21-23$ & $<4.60$ & & $<0.70$ & & $>60.00$ & $<0.66$ & & $<0.030$ & $<0.15$ & & $<1.90$ \\
\hline $56.2-5-6,7$ & $<4.60$ & & $<0.70$ & & 46.30 & $<0.66$ & & 0.071 & $<0.15$ & & $<1.90$ \\
\hline $56.2-6-2,7$ & $<4.60$ & & $<0.70$ & & 44.60 & $<0.66$ & & $<0.030$ & $<0.15$ & & $<1.90$ \\
\hline $56.2-6-4,7-9$ & $<4.60$ & & $<0.70$ & & 25.40 & $<0.66$ & & 0.030 & $<0.15$ & & $<1.90$ \\
\hline $56.2-6-6,16-18$ & $<4.60$ & & $<0.70$ & & 51.30 & $<0.66$ & & 0.031 & $<0.15$ & & $<1.90$ \\
\hline $56.2-7-2,43-45$ & $<4.60$ & & $<0.70$ & & 49.50 & $<0.66$ & & 0.035 & $<0.15$ & & $<1.90$ \\
\hline $56.2-7-4,39-41$ & $<4.60$ & & $<0.70$ & & 55.00 & $<0.66$ & & $<0.030$ & $<0.15$ & & $<1.90$ \\
\hline $56.2-7-6,7$ & $<4.60$ & & $<0.70$ & & 40.00 & $<0.66$ & & $<0.030$ & $<0.15$ & & $<1.90$ \\
\hline $56.2-8-2,7-9$ & $<4.60$ & & $<0.70$ & & 55.00 & $<0.66$ & & $<0.030$ & $<0.15$ & & $<1.90$ \\
\hline $56.2-8-4,7$ & $<4.60$ & & $<0.70$ & & 47.30 & $<0.66$ & & 0.068 & $<0.15$ & & $<1.90$ \\
\hline $56.2-9-2,7-9$ & $<4.60$ & & $<0.70$ & & 47.80 & $<0.66$ & & 0.041 & 0.20 & & $<1.90$ \\
\hline $56.2-9-4,19$ & $<4.60$ & & $<0.70$ & & 49.00 & $<0.66$ & & 0.076 & 0.33 & & $<1.90$ \\
\hline $56.2-9-6,8-10$ & $<4.60$ & & $<0.70$ & & $>60.00$ & 0.71 & & 0.057 & 0.29 & & $<1.90$ \\
\hline $56.2-10-2,10$ & $<4.60$ & & $<0.70$ & & 46.80 & $<0.66$ & & 0.034 & 0.27 & & $<1.90$ \\
\hline $56.2-10-4,19-21$ & $<5.62$ & & $<0.70$ & & 44.60 & 0.79 & & 0.052 & 0.35 & & $<1.90$ \\
\hline $56.2-10-6,13$ & 16.80 & 5.83 & 2.88 & 7.38 & 39.80 & 1.48 & 26.89 & 0.068 & 0.39 & 8.10 & 3.16 \\
\hline 57.0 Basalt & 49.60 & & 15.70 & & 10.90 & 5.69 & & 0.180 & 2.63 & & 12.50 \\
\hline $57.0-1-1$, Top 5 & 12.60 & & $<0.70$ & & 39.80 & 1.68 & & 0.049 & 0.32 & & 2.09 \\
\hline $57.1-1-2,0-4$ & $<4.60$ & & $<0.70$ & & $>60.00$ & 0.68 & & 0.069 & $<0.15$ & & $<1.90$ \\
\hline $57.1-1-2,13$ & $<4.60$ & & $<0.70$ & & 51.40 & $<0.66$ & & $<0.030$ & $<0.15$ & & $<1.90$ \\
\hline $57.1-1-3,7-9$ & $<4.60$ & & $<0.70$ & & $>60.00$ & $<0.66$ & & $<0.030$ & $<0.15$ & & $<1.90$ \\
\hline $57.1-1-4,7$ & $<4.60$ & & $<0.70$ & & 46.80 & $<0.66$ & & $<0.030$ & $<0.15$ & & $<1.90$ \\
\hline $57.1-1-5,7-9$ & $<4.60$ & & $<0.70$ & & $>60.00$ & $<0.66$ & & $<0.030$ & $<0.15$ & & $<1.90$ \\
\hline $57.1-1-6,11$ & $<4.60$ & & $<0.70$ & & 51.40 & $<0.66$ & & $<0.030$ & $<0.15$ & & $<1.90$ \\
\hline $57.1-2-1,44-46$ & 11.50 & & $<0.70$ & & 46.70 & 1.23 & & 0.058 & 0.36 & & 1.93 \\
\hline $57.1-3-1,22$ & $<4.60$ & & $<0.70$ & & 47.80 & 2.69 & 69.28 & 0.085 & 0.23 & 7.39 & $<1.90$ \\
\hline $57.1-3-2,109-110$ & 11.75 & & 2.00 & & 47.40 & 1.52 & & 0.080 & 0.51 & & 2.34 \\
\hline $57.1-4-1,86$ & 12.30 & 10.79 & 1.14 & 2.78 & 34.00 & 2.46 & 13.82 & 0.095 & 0.41 & 8.07 & 3.31 \\
\hline $57: 1-4-2,20-22$ & 6.31 & & $<0.70$ & & 44.60 & 1.41 & & 0.073 & 0.31 & & 2.29 \\
\hline $57.1-4-3,14$ & 6.62 & & $<0.70$ & & 45.60 & 0.85 & 53.65 & 0.120 & 0.29 & 7.03 & 2.04 \\
\hline $57.1-4-4,145$ & 6.76 & & 0.70 & & 51.30 & 1.86 & 27.58 & 0.130 & 0.27 & 7.48 & 2.02 \\
\hline $57.1-4-5,145$ & 25.70 & & 4.27 & & 36.30 & 4.26 & & 0.071 & 0.88 & & 6.80 \\
\hline
\end{tabular}


TABLE 7 - Continued

\begin{tabular}{|c|c|c|c|c|c|c|c|c|c|c|c|}
\hline Sample Designation & $\begin{array}{c}\mathrm{SiO}_{2} \\
(\%)\end{array}$ & $\begin{array}{r}\mathrm{SiO}_{2} / \\
\mathrm{Al}_{2} \mathrm{O}_{3}\end{array}$ & $\begin{array}{c}\mathrm{Al}_{2} \mathrm{O}_{3} \\
(\%)\end{array}$ & $\begin{array}{c}\mathrm{Al}_{2} \mathrm{O}_{3} / \\
\mathrm{TiO}_{2}\end{array}$ & $\begin{array}{r}\mathrm{CaO} \\
(\%)\end{array}$ & $\begin{array}{l}\mathrm{MgO} \\
(\%)\end{array}$ & $\mathrm{CaO} / \mathrm{MgO}$ & $\begin{array}{c}\mathrm{MnO} \\
(\%)\end{array}$ & $\begin{array}{c}\mathrm{TiO}_{2} \\
(\%)\end{array}$ & $\begin{array}{c}\mathrm{Fe}_{2} \mathrm{O}_{3} / \\
\mathrm{TiO}_{2}\end{array}$ & $\begin{array}{c}\mathrm{Fe}_{2} \mathrm{O}_{3} \\
(\%)\end{array}$ \\
\hline $57.2-1-1,22-24$ & $<4.60$ & & $<0.70$ & & $>60.00$ & $<0.66$ & & 0.030 & $<0.15$ & & $<1.90$ \\
\hline $58.1-1-1,139$ & 22.70 & 4.69 & 4.84 & 13.83 & 33.50 & 2.04 & 16.42 & $<0.030$ & 0.35 & 9.89 & 3.46 \\
\hline $58.1-1-2,91$ & $<4.60$ & & $<0.70$ & & 42.60 & 0.75 & 5.68 & 0.140 & $<0.15$ & & $<1.90$ \\
\hline $58.1-1-3,145-150$ & $<4.60$ & & $<0.70$ & & $>60.00$ & 3.20 & & $<0.030$ & $<0.15$ & & $<1.90$ \\
\hline $58.2-1-2,16$ & 14.20 & 5.53 & 2.57 & 4.21 & 38.00 & 1.51 & 25.17 & 0.130 & 0.61 & 5.12 & 3.16 \\
\hline $58.2-1-4,31$ & 28.80 & 4.26 & 6.76 & 6.76 & 25.70 & 3.32 & 7.74 & 0.100 & 1.00 & 6.10 & 6.10 \\
\hline $58.2-1-5,0$ & 44.20 & & 16.00 & & 1.29 & 4.73 & & 0.140 & 1.66 & & $>10.00$ \\
\hline $58.2-1-5,1$ & 45.70 & 4.27 & 10.70 & 6.29 & 12.00 & 6.92 & 1.73 & 0.150 & 1.70 & & $<10.00$ \\
\hline $58.2-1-5,26-28$ & 22.90 & & 3.89 & & 38.90 & 1.62 & & 0.229 & 0.46 & & 2.95 \\
\hline $58.2-1-6,95$ & 50.60 & 3.97 & 12.75 & 9.81 & 6.10 & 2.40 & 2.54 & 0.180 & 1.66 & & 10.00 \\
\hline $58.2-1-8,16-18$ & $<4.60$ & & $<0.70$ & & $>60.00$ & $<0.66$ & & 0.098 & $<0.15$ & & $<1.90$ \\
\hline $58.9-1-5,0-3$ & 50.00 & & 13.50 & & 13.50 & 5.57 & & 0.132 & 1.84 & & $>10.00$ \\
\hline $59.1-3-\mathrm{CC}$ & 46.80 & 2.93 & 16.00 & 10.32 & 1.78 & 3.63 & 0.49 & $>0.210$ & 1.55 & 6.58 & 10.20 \\
\hline $59.1-3-1,130$ & 44.30 & 2.95 & 15.00 & 12.50 & 2.57 & 3.98 & 0.65 & $>0.210$ & 1.20 & 7.08 & 8.50 \\
\hline $59.1-3-3,7$ & 42.60 & 2.34 & 18.20 & 12.64 & 1.82 & 3.43 & 0.53 & $>0.210$ & 1.44 & & 10.00 \\
\hline $59.1-3-3,146-148$ & 66.90 & & 5.25 & & 0.88 & 1.60 & & 0.036 & 0.36 & & 2.72 \\
\hline $59.1-3-2,11-13$ & 49.50 & & 15.30 & & 2.95 & 3.68 & & $>0.210$ & 1.12 & & 8.32 \\
\hline $59.2-1-1,47$ & 45.70 & 3.91 & 11.70 & 5.63 & 3.93 & 5.38 & 0.73 & $>0.210$ & 2.08 & & 10.00 \\
\hline $59.2-1-2,4-6$ & 45.70 & & 10.70 & & 4.07 & 4.57 & & $>0.210$ & $>2.25$ & & $>10.00$ \\
\hline $59.2-1-3,4$ & 47.80 & 4.27 & 11.20 & 6.02 & 5.50 & 5.00 & 1.10 & $>0.210$ & 1.86 & 4.89 & 9.10 \\
\hline $59.2-1-\mathrm{CC}$ & 49.50 & 4.42 & 11.20 & 5.33 & 3.51 & 3.98 & 0.88 & $>0.210$ & 2.10 & 4.34 & 9.12 \\
\hline $59.2-2-2,145$ & 49.50 & 4.95 & 10.00 & 5.75 & 4.27 & 5.75 & 0.74 & $>0.210$ & 1.74 & 4.78 & 8.31 \\
\hline $59.2-2-3,7-9$ & 43.60 & & 10.48 & & 5.76 & 4.17 & & $>0.210$ & $>2.25$ & & $>10.00$ \\
\hline $59.2-2-4,4$ & 46.70 & 5.07 & 9.22 & 3.84 & 4.57 & 4.36 & 1.05 & $>0.210$ & 2.40 & 3.54 & 8.50 \\
\hline $59.2-2-5,35-37$ & 46.30 & & 10.00 & & 3.89 & 3.80 & & $>0.210$ & $>2.25$ & & $>10.00$ \\
\hline $59.2-2-6,16$ & 49.50 & 6.11 & 8.10 & 4.35 & 4.36 & 4.90 & 0.85 & $>0.210$ & 1.86 & 4.27 & 7.95 \\
\hline $59.2-2-\mathrm{CC}$ & 50.60 & 4.96 & 10.20 & 63.75 & 4.48 & 5.00 & 0.90 & $>0.210$ & 0.16 & 58.38 & 9.34 \\
\hline 59.2-3, Top & 50.00 & 4.07 & 12.30 & 5.62 & 3.51 & 4.26 & 0.82 & $>0.210$ & 2.19 & & 5.30 \\
\hline $59.2-3-1,145$ & 40.40 & 4.34 & 9.30 & 5.05 & 5.01 & 3.23 & 1.55 & $>0.210$ & 1.84 & 5.31 & 9.77 \\
\hline $59.2-3-2,145-150$ & 44.30 & & 12.30 & & 4.68 & 3.98 & & $>0.210$ & $>2.25$ & & $>10.00$ \\
\hline $59.2-3-\mathrm{CC}$ & 50.00 & 3.62 & 13.80 & 10.22 & 2.98 & 4.13 & 0.72 & $>0.210$ & 1.35 & 2.24 & 9.77 \\
\hline $59.2-4-1,66$ & 42.60 & 3.20 & 13.30 & 7.73 & 4.62 & 4.32 & 1.07 & $>0.210$ & 1.72 & 5.06 & 8.71 \\
\hline $59.2-4-\mathrm{CC}$ & 45.70 & 2.86 & 16.00 & 8.42 & 3.85 & 3.85 & 1.00 & $>0.210$ & 1.90 & & $>10.50$ \\
\hline $59.2-5-1,89$ & 46.30 & 4.79 & 9.66 & 20.13 & 4.07 & 3.24 & 1.26 & $>0.210$ & 0.48 & 9.31 & 4.47 \\
\hline $59.2-5-\mathrm{CC}$ & 51.90 & 5.37 & 9.67 & 7.67 & 1.87 & 3.80 & 0.49 & $>0.210$ & 1.26 & 4.73 & 5.96 \\
\hline 59.2-6, Тop & 52.50 & 3.80 & 13.80 & 28.37 & 2.45 & 3.39 & 0.72 & 0.210 & 0.49 & 9.78 & 4.79 \\
\hline 59.2-6, Top & 42.60 & 3.77 & 11.30 & 9.58 & 9.34 & 4.95 & 1.89 & 0.140 & 1.18 & 8.29 & 9.78 \\
\hline 59.2-6, Тop & 38.04 & 4.68 & 8.12 & 16.57 & 3.02 & 3.71 & 0.81 & $>0.210$ & 0.49 & 7.41 & 3.63 \\
\hline $59.2,6$ & 46.80 & & 8.92 & & 1.88 & 3.51 & & $>0.210$ & 0.71 & & $>10.00$ \\
\hline $59.2-6-2,0$ & 42.20 & 4.67 & 9.04 & 6.55 & 5.07 & 3.63 & 1.40 & $>0.210$ & 1.38 & 5.62 & 7.76 \\
\hline $60.0-1-1,7-9$ & 30.20 & & 8.92 & & 22.62 & 2.66 & & 0.059 & 0.46 & & 5.62 \\
\hline $60.0-1-2,9$ & 14.80 & 2.82 & 5.24 & 16.90 & 42.20 & 1.27 & 33.23 & 0.110 & 0.31 & 9.74 & 3.02 \\
\hline $60.0-1-3,10$ & 17.00 & 3.24 & 5.24 & 18.07 & 36.70 & 0.83 & 44.22 & 0.130 & 0.29 & 9.28 & 2.69 \\
\hline $60.0-1-\mathrm{CC}$ & 38.00 & 4.27 & 8.90 & 19.35 & 17.40 & 3.16 & 5.50 & 0.068 & 0.46 & 11.41 & 5.25 \\
\hline $60.0-2-1,87-89$ & 28.50 & & 8.82 & & 26.15 & 1.50 & & 0.133 & 0.35 & & 3.24 \\
\hline $60.0-2-2,12$ & 38.90 & 3.89 & 10.00 & 23.81 & 21.60 & 2.09 & 10.33 & 0.100 & 0.42 & 10.88 & 4.57 \\
\hline $60.0-2-\mathrm{CC}$ & 48.90 & 3.76 & 13.00 & 20.63 & 7.59 & 1.74 & 4.36 & 0.150 & 0.63 & 8.73 & 5.50 \\
\hline $60.0-3-1,117$ & 53.70 & 4.40 & 12.20 & 35.88 & 4.57 & 1.15 & 3.97 & 0.130 & 0.34 & 9.41 & 3.20 \\
\hline
\end{tabular}


TABLE 7 - Continued

\begin{tabular}{|c|c|c|c|c|c|c|c|c|c|c|c|}
\hline Sample Designation & $\begin{array}{c}\mathrm{SiO}_{2} \\
(\%)\end{array}$ & $\begin{array}{c}\mathrm{SiO}_{2} \\
\mathrm{Al}_{2} \mathrm{O}_{3}\end{array}$ & $\begin{array}{c}\mathrm{Al}_{2} \mathrm{O}_{3} \\
(\%)\end{array}$ & $\begin{array}{c}\mathrm{Al}_{2} \mathrm{O}_{3} / \\
\mathrm{TiO}_{2}\end{array}$ & $\begin{array}{r}\mathrm{CaO} \\
(\%)\end{array}$ & $\begin{array}{c}\mathrm{MgO} \\
(\%)\end{array}$ & $\mathrm{CaO} / \mathrm{MgO}$ & $\begin{array}{c}\mathrm{MnO} \\
(\%)\end{array}$ & $\begin{array}{c}\mathrm{TiO}_{2} \\
(\%)\end{array}$ & $\begin{array}{c}\mathrm{Fe}_{2} \mathrm{O}_{3} / \\
\mathrm{TiO}_{2}\end{array}$ & $\begin{array}{c}\mathrm{Fe}_{2} \mathrm{O}_{3} \\
(\%)\end{array}$ \\
\hline $60.0-3-2,8-16$ & 47.80 & & 12.60 & & 6.93 & 3.05 & & 0.170 & 0.91 & & 8.62 \\
\hline $60.0-3-\mathrm{CC}$ & 49.00 & 3.71 & 13.20 & 17.60 & 6.82 & 3.72 & 1.83 & 0.150 & 0.75 & 11.33 & 8.50 \\
\hline $60.0-4-\mathrm{CC}$ & 57.10 & 3.81 & 15.00 & 28.85 & 6.38 & 2.57 & 2.48 & 0.200 & 0.52 & 13.63 & 7.09 \\
\hline $60.0-4-1,145$ & 49.60 & 4.13 & 12.00 & 20.69 & 8.62 & 2.40 & 3.59 & 0.160 & 0.58 & 11.52 & 6.68 \\
\hline $60.0-4-1,145$ & 52.50 & 3.55 & 14.80 & 28.46 & 10.50 & 1.78 & 5.90 & 0.170 & 0.52 & 11.60 & 6.03 \\
\hline $60.0-4-2,145$ & 49.50 & 3.67 & 13.50 & 18.75 & 4.22 & 2.21 & 1.91 & 0.180 & 0.72 & 8.56 & 6.16 \\
\hline $60.0-5-1,51-53$ & 51.80 & & 10.70 & & 5.00 & 0.93 & & 0.106 & 0.33 & & 3.02 \\
\hline $60.0-5-2,26$ & 57.70 & 5.43 & 9.34 & 23.35 & 6.54 & 0.65 & 10.06 & 0.090 & 0.40 & 8.38 & 3.35 \\
\hline $60.0-5-3,40-42$ & 51.20 & & 11.50 & & 5.24 & 1.23 & & 0.116 & 0.48 & & 4.07 \\
\hline $60.0-5-\mathrm{CC}$ & 60.30 & 4.45 & 13.55 & 23.77 & 5.05 & 1.80 & 2.81 & 0.210 & 0.57 & 9.86 & 5.62 \\
\hline $60.0-6-1,86$ & 51.30 & 3.98 & 12.90 & 19.85 & 9.11 & 2.48 & 3.67 & 0.190 & 0.65 & 12.51 & 8.13 \\
\hline $60.0-6-2,16-18$ & 51.80 & & 13.18 & & 9.43 & 2.45 & & 0.188 & 0.81 & & 8.43 \\
\hline $60.0-6-3,14$ & 4.31 & 3.06 & 14.10 & 16.59 & 11.70 & 1.80 & 6.50 & 0.170 & 0.85 & 9.02 & 7.67 \\
\hline $60.0-6-4,25-27$ & 50.60 & & 14.10 & & 9.78 & 2.57 & & 0.138 & 0.65 & & 7.95 \\
\hline $60.0-6-5,37$ & 53.70 & 4.33 & 12.40 & 22.96 & 5.44 & 1.05 & 5.18 & 0.140 & 0.54 & 8.09 & 4.37 \\
\hline $60.0-6-6,26$ & 55.00 & & 12.89 & & 9.12 & 1.72 & & 0.138 & 0.65 & & 6.38 \\
\hline $60.0-6-7,40$ & 63.10 & 4.89 & 12.90 & 26.88 & 5.36 & 1.27 & 4.22 & 0.160 & 0.48 & 8.69 & 4.77 \\
\hline $60.0-7-1,81$ & 60.30 & & 11.10 & & 5.68 & 1.25 & & 0.132 & 0.54 & & 5.25 \\
\hline $60.0-7-2,7$ & 52.50 & 4.41 & 11.90 & 24.28 & 5.62 & 0.80 & 6.99 & 0.200 & 0.49 & 8.61 & 4.22 \\
\hline $60.0-8-1,145$ & 50.60 & & 12.75 & & 6.03 & 2.45 & & 0.168 & 0.89 & & 7.95 \\
\hline $60.0-8-2,72$ & 49.00 & 3.80 & 12.90 & 17.92 & 5.82 & 2.82 & 2.06 & $>0.210$ & 0.72 & 9.50 & 6.34 \\
\hline $60.0-8-3,145$ & 53.70 & & 12.16 & & 5.55 & 2.34 & & 0.166 & 0.69 & & 6.04 \\
\hline $60.0-9-2,145$ & 49.60 & 3.52 & 21.04 & 21.04 & 5.25 & 2.11 & 2.49 & 0.190 & 0.67 & 9.64 & 6.46 \\
\hline $60.0-9-5,16-18$ & 51.80 & & 13.32 & & 7.33 & 2.51 & & 0.143 & 0.76 & & 7.00 \\
\hline
\end{tabular}


TABLE 8

Results of Determination of Amorphous Silica

\begin{tabular}{|c|c|c|c|c|c|}
\hline Sample Designation & $\begin{array}{c}\mathrm{SiO}_{2} \\
(\%)\end{array}$ & Sample Designation & $\begin{array}{c}\mathrm{SiO}_{2} \\
(\%)\end{array}$ & Sample Designation & $\begin{array}{c}\mathrm{SiO}_{2} \\
(\%)\end{array}$ \\
\hline $44.0-1-1,145$ & 0.05 & $47.1-1-1,145$ & 0.46 & $47.2-13-4,145$ & 0.21 \\
\hline $44.0-1-2,145$ & 0.00 & $47.2-1-3,11$ & 1.48 & $47.2-13-4,145$ & 0.17 \\
\hline $44.0-1-3,145$ & 0.05 & $47.2-1-4,7$ & 1.37 & $47.2-13-5,145$ & 0.18 \\
\hline $44.0-1-3$ & 0.33 & $47.2-2-2,47$ & 1.72 & $47.2-14-\mathrm{CC}$ & Tr. \\
\hline 44.0-1-3, Mixed & 0.00 & $47.2-2-3,7$ & 1.12 & $47.2-14-1,145$ & 0.00 \\
\hline $44.0-1-4,145$ & 0.09 & $47.2-2-5,5$ & 1.52 & $47.2-14-2,145$ & 0 \\
\hline $44.0-2-\mathrm{CC}$ & Tr. & $47.2-3-\mathrm{CC}$ & 0.86 & $47.2-14-3,145$ & Tr. \\
\hline $44.0-2-1,145$ & 0.07 & $47.2-3-2,6.5$ & 1.10 & $47.2-14-5,145$ & Tr. \\
\hline $44.0-2-2,145$ & 0.10 & $47.2-3-4,16$ & 1.38 & $48.1-1-1,26$ & 1.14 \\
\hline $44.0-2-3,145$ & 0.04 & $47.2-4-\mathrm{CC}$ & 0.91 & $48.2-1-\mathrm{CC}$ & 0.12 \\
\hline $44.0-2-4,145$ & 0.05 & $47.2-4-2,17$ & 0.82 & $48.2-1-1,28$ & 0.64 \\
\hline $44.0-2-5,145$ & 0.12 & $47.2-4-4,16.5$ & 0.94 & $48.2-1-3,16$ & 0.73 \\
\hline $44.0-2-6,145$ & 0.00 & $47.2-4-6,17$ & 0.84 & $48.2-1-5,14.5$ & 0.83 \\
\hline $44.0-3-\mathrm{CC}$ & 2.42 & $47.2-5-2,6$ & 0.74 & $48.2-2-\mathrm{CC}$ & 0.07 \\
\hline $44.0-3-2,145$ & 0.00 & $47.2-5-4,6$ & 1.22 & $48.2-2-1,145$ & 0.06 \\
\hline $44.0-3-3,145$ & 0.00 & $47.2-5-6,6.5$ & 0.75 & $48.2-2-2,17$ & 0.08 \\
\hline $44.0-3-4,145$ & 0.00 & $47.2-6-3,17$ & 0.54 & $48.2-2-4,18$ & 0.06 \\
\hline $44.0-3-5,145$ & 3.53 & $47.2-7-2,17$ & 0.14 & $48.2-2-6,16$ & $\operatorname{Tr}$ \\
\hline $44.0-3-5,145$ & 3.56 & $47.2-7-4,17$ & 0.16 & $48.2-3-2 \quad 28$ & 1.71 \\
\hline $44.0-4-\mathrm{CC}$ & 0.05 & $47.2-7-6,126$ & 0.22 & $48.2-3-4,17$ & 2.11 \\
\hline 44.0-4-0, Top & 0.00 & $47.2-8-2,17$ & 0.04 & $49.0-1-1,76$ & 1.16 \\
\hline $44.0-4-2,145$ & Tr. & $47.2-8-4,17$ & 0.15 & $49.0-1-6,7$ & 3.33 \\
\hline $44.0-4-3,145$ & 0.00 & $47.2-8-6-\mathrm{CC}$ & 0.15 & $49.1-1-2,16$ & 1.15 \\
\hline $44.0-4-4,145$ & 0.00 & $47.2-8-6,16$ & 0.00 & $49.1-1-3,22$ & 1.27 \\
\hline $44.0-5-0$ & 1.35 & $47.2-9-\mathrm{CC}$ & 0.36 & $49.1-1-4,117$ & 1.10 \\
\hline $44.0-4-5,145$ & 0.00 & $47.2-9-1,150$ & 0.18 & $49.1-1-5,60$ & 2.52 \\
\hline $44.0-4-6,145$ & 0.00 & $47.2-9-2,17$ & 0.21 & $49.1-1-5,60$ & 0.21 \\
\hline $44.1-1-\mathrm{CC}$ & 0.95 & $47.2-9-4,0$ & 0.19 & $49.1-2-1,145$ & 0.61 \\
\hline $45.1-1-1,70$ & 1.10 & $47.2-9-5,17$ & 0.11 & $49.1-2-3,145$ & 3.52 \\
\hline $45.1-1-1,7$ & 1.73 & $47.2-9-5,150$ & 0.16 & $50.0-2-\mathrm{CC}$ & 0.97 \\
\hline $45.1-1-1,145$ & 1.51 & $47.2-9-6,0$ & 0.25 & $50.0-2-1,145$ & 0.77 \\
\hline $45.1-1-2,100$ & 2.48 & $47.2-10-\mathrm{CC}$ & 0.23 & $50.0-2-3,150$ & 2.09 \\
\hline $45.1-1-2,124$ & 1.27 & $47.2-10$ & 0.05 & $50.0-2-4,150$ & 0.82 \\
\hline $45.1-1-3,23$ & 1.34 & $47.2-10-1,17$ & 0.08 & $50.0-2-6,150$ & 1.65 \\
\hline $45.1-1-4,22$ & 1.88 & $47.2-10-3,18$ & 0.03 & $50.1-1-3,7$ & 1.80 \\
\hline $45.1-1-4,136$ & 1.34 & $47.2-10-5,17$ & 0.12 & $50.1-1-3,7$ & 1.62 \\
\hline $45.1-1-5,4$ & 1.19 & $47.2-11-\mathrm{CC}$ & 0.03 & $50.1-1-5,17$ & 2.95 \\
\hline $45.1-2-\mathrm{CC}$ & 31.10 & $47.2-11-1,145$ & Tr. & $50.1-2-1,130$ & 1.50 \\
\hline $45.1-2-\mathrm{CC}$ & 27.64 & $47.2-11-3,145$ & 0.19 & $50.1-2-3,35$ & 1.90 \\
\hline 46.0 & 19.44 & $47.2-11-4,145$ & 0.27 & $50.1-2-5,8$ & 2.22 \\
\hline $46.0-1-2,145$ & 1.51 & $47.2-11-5,145$ & Tr. & $50.1-2-6,150$ & 1.50 \\
\hline $46.0-1-4,145$ & 2.44 & $47.2-11-6,145$ & 0.21 & $50.1-3-3,15$ & 1.14 \\
\hline $46.0-1-6$ & 31.96 & $47.2-12-\mathrm{CC}$ & 0.05 & $50.1-3-5,28$ & 1.36 \\
\hline $47.0-1-2,5$ & 2.67 & $47.2-12-1,145$ & 0.16 & $51.0-1-\mathrm{CC}$ & 1.01 \\
\hline $47.0-1-3,16$ & 11.88 & $47.2-12-3,145$ & 0.00 & $51.0-1-1,11$ & 1.52 \\
\hline $47.0-1-4,95$ & 2.15 & $47.2-13-\mathrm{CC}$ & Tr. & $51.1-1-3,30$ & 2.82 \\
\hline $47.0-1-5,3.5$ & 1.76 & $47.2-13-1,145$ & 0.00 & $51.1-1-4,30$ & 4.26 \\
\hline 47.1-1, & 0.24 & $47.2-13-3,16$ & 0.03 & $51.1-1-5,16$ & 3.77 \\
\hline
\end{tabular}


TABLE 8 - Continued

\begin{tabular}{|c|c|c|c|c|c|}
\hline Sample Designation & $\begin{array}{c}\mathrm{SiO}_{2} \\
(\%)\end{array}$ & Sample Designation & $\begin{array}{c}\mathrm{SiO}_{2} \\
(\%)\end{array}$ & Sample Designation & $\begin{array}{c}\mathrm{SiO}_{2} \\
(\%)\end{array}$ \\
\hline $51.0-1-3,6$ & 1.35 & $54.0-2-1,17$ & 2.37 & $55.0-13-3,40$ & 1.43 \\
\hline $51.0-1-5,15$ & 1.38 & $54.0-2-4,16$ & 2.89 & $55.0-13-5,7$ & 1.13 \\
\hline $51.1-1-1,15$ & 2.55 & $54.0-3-1,140$ & 3.24 & $55.0-14-2,145$ & 0.65 \\
\hline $51.1-1-3,30$ & 2.82 & $54.0-4-2,14$ & 2.90 & $55.0-14-4,145$ & 1.10 \\
\hline $51.1-1-4,30$ & 4.26 & $54.0-6-3-23,25$ & 2.96 & $55.0-14-6,145$ & 1.20 \\
\hline $51.1-1-5,16$ & 3.77 & $54.0-6-4,78$ & 3.74 & $56.2-1-6,7$ & 1.15 \\
\hline $51.1-2-1,127$ & 1.24 & $54.0-7-17,138$ & 3.18 & $56.2-1-2,145$ & 0.26 \\
\hline $51.1-10-1,145$ & 1.21 & $54.0-7-8,16$ & 2.03 & $56.2-2-4,36$ & 0.15 \\
\hline $51.0-1-1,27$ & 1.60 & 54.0-9-1, Basalt & 3.19 & $56.2-3-2,145$ & 0.26 \\
\hline $52.0-1-3,8$ & 1.85 & $55.0-1-2,7$ & 0.71 & $56.2-3-6,145$ & 0.28 \\
\hline $52.0-2-\mathrm{CC}$ & 1.70 & $55.0-1-2,30$ & 0.84 & $56.2-3-6,145$ & 0.26 \\
\hline $52.0-2-2,7$ & 2.08 & $55.0-1-4,7$ & 0.64 & $56.2-4-4,14$ & 0.27 \\
\hline $52.0-2-6,145$ & 1.25 & $55.0-1-6,7$ & 0.51 & $56.2-5-6,7$ & 0.66 \\
\hline $52.0-4-3,5$ & 2.30 & $55.0-2-2,7$ & 0.50 & $56.2-6-2,7$ & 0.94 \\
\hline $52.0-5-2,0$ & 2.11 & $55.0-2-4,15$ & 0.32 & $56.2-7-6,7$ & 1.25 \\
\hline $52.0-5-4,18$ & 1.23 & $55.0-2-4,15$ & 0.54 & $56.2-8-4 \quad 7$ & 1.17 \\
\hline $52.0-6-\mathrm{CC}$ & 1.44 & $55.0-2-5,70$ & 0.48 & $56.2-9-4,19$ & 1.00 \\
\hline $52.0-7-\mathrm{CC}$ & 0.75 & $55.0-3-1,16$ & 0.36 & $56.2-10-2,10$ & 0.96 \\
\hline $52.0-8-1,145$ & 21.12 & $55.0-3-4,145$ & 0.45 & $56.2-10-6,13$ & 1.39 \\
\hline $52.0-8-4,145$ & 9.55 & $55.0-4-1,13$ & 0.33 & $57.1-3-1,17$ & 1.10 \\
\hline $52.0-8-5,145$ & 14.89 & $55-0-4-3,7$ & 0.28 & $57.1-4-4,145$ & 1.56 \\
\hline $52.0-9-\mathrm{CC}$ & 20.54 & $55.0-4-5,7$ & 0.19 & $58.2-1-6,95$ & 4.06 \\
\hline $52.0-10-\mathrm{CC}$ & 3.14 & $55.0-4-1,7$ & 0.13 & $59.1-3-\mathrm{CC}$ & 1.71 \\
\hline $53.0-2-\mathrm{CC}$ & 1.38 & $55.0-5-5,7$ & 0.36 & $59.2-3-\mathrm{CC}$ & 2.22 \\
\hline $53.0-4-2$ & 2.76 & $55.0-6-3,17$ & 0.52 & $59.2-5-\mathrm{CC}$ & 11.26 \\
\hline 53.0-6-CC & 1.18 & $55.0-7-1,14$ & 0.60 & $59.2-6-$ & 3.49 \\
\hline $53.0-8-\mathrm{CC}$ & 0.72 & $55-0-7-5,19$ & 1.03 & $59.2-6$ & 2.20 \\
\hline $53.1-2-2,145$ & 1.22 & $55-0-8-3,16$ & 1.28 & $60.0-1-\mathrm{CC}$ & 1.40 \\
\hline $53.1-3-1,150$ & 2.22 & $55.0-9$ & 1.06 & 60.0-3-СC, Тор & 2.25 \\
\hline $53.1-3-2,40$ & 2.44 & $55.0-10-2,10$ & 2.06 & $60.0-4-\mathrm{CC}$ & 1.68 \\
\hline $54.0-1-1,5$ & 2.39 & $55.0-10-5,16$ & 3.34 & $60.0-4-1,145$ & 1.91 \\
\hline $54.0-1-2,7$ & 0.66 & $55.0-12-3,8$ & 2.60 & $60.0-4-2,145$ & 2.82 \\
\hline $54.0-1-2,20$ & 2.53 & $55.0-13-1,7$ & 2.06 & $60.0-5-\mathrm{CC}$ & 2.77 \\
\hline
\end{tabular}


TABLE 9

Results of Analysis of $\mathrm{CO}_{2}$ and Organic Carbon

\begin{tabular}{|c|c|c|c|c|c|c|c|}
\hline Sample Designation & $\begin{array}{c}\mathrm{CO}_{2} \\
(\%)\end{array}$ & $\begin{array}{c}\text { Org. C } \\
(\%)\end{array}$ & $\begin{array}{c}\mathrm{CaCO}_{3} \\
(\%)\end{array}$ & Sample Designation & $\begin{array}{l}\mathrm{CO}_{2} \\
(\%)\end{array}$ & $\underset{(\%)}{\text { Org. C }}$ & $\begin{array}{c}\mathrm{CaCO}_{3} \\
(\%)\end{array}$ \\
\hline $44.0-1-1,145$ & 41.93 & 0.20 & 95.35 & $47.2-2-2,47$ & 27.64 & 0.18 & 62.92 \\
\hline $44.0-1-2,145$ & 42.26 & 0.16 & 96.1 & $47.2-2-3,7$ & 36.47 & 0.11 & 82.93 \\
\hline $44.0-1-3,145$ & 42.38 & 0.19 & 96.37 & $47.2-2-5,5$ & 36.12 & 0.15 & 82.14 \\
\hline $44.0-1-3,50$ & 42.22 & 0.06 & 96.01 & $47.2-2-5,5$ & 36.04 & 0.17 & 81.95 \\
\hline $44.0-1-3$, total & 42.34 & 0.05 & 96.28 & $47.2-3-\mathrm{CC}$ & 38.42 & 0.11 & 87.37 \\
\hline $44.0-1-4,145$ & 42.20 & 0.16 & 95.96 & $47.2-3-2,6.5$ & 33.40 & 0.15 & 75.95 \\
\hline $44.0-2-\mathrm{CC}$ & 42.69 & 0.06 & 97.08 & $47.2-3-4,16$ & 37.10 & 0.05 & 84.37 \\
\hline $44.0-2-1,145$ & 41.48 & 0.27 & 94.33 & $47.2-4-2,17$ & 39.40 & 0.05 & 89.6 \\
\hline $44.0-2-2,145$ & 41.71 & 0.04 & 94.85 & $47.2-4-4,16.5$ & 36.95 & 0.10 & 84.02 \\
\hline $44.0-2-3,145$ & 42.00 & 0.05 & 92.51 & $47.2-4-6,17$ & 38.90 & 0.04 & 88.96 \\
\hline $44.0-2-4,145$ & 42.09 & 0.08 & 95.71 & $47.2-4-\mathrm{CC}$ & 37.32 & 0.18 & 84.87 \\
\hline $44.0-2-5,145$ & 41.53 & 0.07 & 94.44 & $47.2-5-2,6$ & 40.18 & 0.05 & 91.37 \\
\hline $44.0-2-6,145$ & 42.21 & 0.12 & 95.99 & $47.2-5-4,6$ & 34.85 & 0.05 & 79.25 \\
\hline $44.0-3-\mathrm{CC}$ & 32.78 & 0.35 & 74.54 & $47.2-5-6,6.5$ & 37.94 & 0.05 & 86.28 \\
\hline $44.0-3-2,145$ & 41.28 & 0.15 & 93.87 & $47.2-6-3,17$ & 37.55 & 0.05 & 85.39 \\
\hline $44.0-3-3,145$ & 42.25 & 0.08 & 96.08 & $47.2-7-2,17$ & 41.88 & 0.16 & 95.24 \\
\hline $44.0-3-4,145$ & 41.69 & 0.14 & 94.8 & $47.2-7-4,17$ & 41.35 & 0.16 & 94.03 \\
\hline $44.0-3-5,145$ & 35.71 & 0.10 & 81.2 & $47.2-7-6,126$ & 41.97 & 0.14 & 95.44 \\
\hline $44.0-4-\mathrm{CC}$ & 41.51 & 0.12 & 94.39 & $47.2-8-0,17$ & 42.06 & 0.06 & 95.64 \\
\hline 44.0-4-0, Тор & 41.54 & 0.11 & 94.46 & $47.2-8-0,17$ & 42.28 & 0.05 & 96.14 \\
\hline $44.0-4-2,145$ & 42.13 & 0.09 & 95.8 & $47.2-8-4,17$ & 42.36 & 0.04 & 96.33 \\
\hline $44.0-4-3,145$ & 42.10 & & 95.74 & $47.2-8-6-\mathrm{CC}$ & 42.31 & 0.11 & 96.21 \\
\hline $44.0-4-4,145$ & 41.93 & 0.12 & 95.35 & $47.2-8-6,16$ & 42.50 & 0.05 & 96.65 \\
\hline $44.0-4-5,145$ & 42.04 & 0.05 & 95.6 & $47.2-9-\mathrm{CC}$ & 39.73 & 0.16 & 90.35 \\
\hline $44.0-4-6,145$ & 41.61 & 0.19 & 94.62 & $47.2-9-1,150$ & 42.00 & 0.08 & 95.51 \\
\hline $44.0-5-0$ & 36.38 & 0.22 & 82.73 & $47.2-9-2,17$ & 42.08 & 0.03 & 95.69 \\
\hline \multirow[t]{2}{*}{45.1} & 0.04 & 0.03 & 0.09 & $47.2-9-4,0-5$ & 41.76 & 0.11 & 94.96 \\
\hline & 0.07 & 0.03 & 0.16 & $47.2-9-5,17$ & 41.91 & 0.04 & 95.30 \\
\hline $45.1-1-2,124$ & 0.12 & 0.23 & 0.27 & $47.2-9-5,150$ & 41.04 & 0.17 & 93.32 \\
\hline $45.1-1-3,23$ & 0.12 & 0.04 & 0.27 & $47.2-9-6,0-5$ & 41.16 & 0.09 & 93.6 \\
\hline $45.1-1-4,22$ & 0.16 & 0.04 & 0.36 & $47.2-10-\mathrm{CC}$ & 41.37 & 0.09 & 94.08 \\
\hline $45.1-1-4,136$ & 13.51 & 0.14 & 30.79 & $47.2-10$, Top & 41.94 & 0.09 & 95.37 \\
\hline $45.1-1-5,4$ & 4.66 & 0.08 & 10.6 & $47.2-10-1,17$ & 42.94 & 0.17 & 97.65 \\
\hline 46.0 & 0.16 & NIL & 0.36 & $47.2-10-3,18$ & 42.21 & 0.09 & 95.99 \\
\hline $46.0-1-2,145$ & 0.16 & 0.07 & 0.36 & $47.2-10-5,17$ & 41.35 & 0.03 & 94.03 \\
\hline $46.0-1-4,145$ & 0.21 & 0.05 & 0.48 & $47.2-11-\mathrm{CC}$ & 42.42 & 0.03 & 96.46 \\
\hline $46.0-1-6,145$ & 0.15 & 0.03 & 0.34 & $47.2-11-1,145$ & 41.89 & 0.10 & 95.26 \\
\hline $47.0-1-4,25$ & 24.70 & 0.24 & 56.17 & $47.2-11-3,145$ & 41.80 & 0.14 & 95.05 \\
\hline $47.0-1-2,5$ & 22.22 & 0.16 & 50.52 & $47.2-11-4,145$ & 42.52 & 0.03 & 96.69 \\
\hline $47.0-1-3,16$ & & 0.40 & 53.95 & $47.2-11-5,145$ & 42.49 & 0.11 & 96.69 \\
\hline $47.0-1-5,3.5$ & & 0.16 & 74.14 & $47.2-11-6,145$ & 42.58 & 0.07 & 96.83 \\
\hline 47.1-1, Top & 40.70 & 0.10 & 92.55 & $47.2-12-\mathrm{CC}$ & 42.48 & 0.08 & 96.6 \\
\hline $47.1-1-1,145$ & 37.33 & 0.14 & 84.89 & $47.2-12-1,145-150$ & 42.26 & 0.08 & 96.1 \\
\hline $47.2-1-3,11$ & 30.92 & 0.12 & 70.31 & $47.2-12-3,145$ & 42.63 & 0.05 & 96.94 \\
\hline $47.2-1-4,7$ & 32.84 & 0.12 & 74.68 & $47.2-13-\mathrm{CC}$ & 42.87 & 0.05 & 97.49 \\
\hline
\end{tabular}


TABLE 9 - Continued

\begin{tabular}{|c|c|c|c|c|c|c|c|}
\hline Sample Designation & $\begin{array}{c}\mathrm{CO}_{2} \\
(\%)\end{array}$ & $\begin{array}{c}\text { Org. C } \\
(\%)\end{array}$ & $\begin{array}{c}\mathrm{CaCO}_{3} \\
(\%)\end{array}$ & Sample Designation & $\begin{array}{c}\mathrm{CO}_{2} \\
(\%)\end{array}$ & $\begin{array}{c}\text { Org. C } \\
(\%)\end{array}$ & $\begin{array}{c}\mathrm{CaCO}_{3} \\
(\%)\end{array}$ \\
\hline $47.2-13-1,145$ & & 0.03 & 97.4 & $52.0-1-1,27$ & & 0.10 & Nil \\
\hline $47.2-13-3,16$ & & 0.03 & 96.87 & $52.0-1-3,8$ & 0.04 & 0.03 & 0.09 \\
\hline $47.2-13-4,145$ & & 0.05 & 95.99 & $52.0-1-5,10$ & & 0.08 & Nil \\
\hline $47.2-13-5,145$ & 42.70 & 0.03 & 97.1 & $52.0-1-3,8$ & 0.05 & 0.03 & 0.11 \\
\hline $47.2-14-\mathrm{CC}$ & 42.62 & 0.07 & 96.92 & $52.0-2-4,8$ & & 0.07 & 0.16 \\
\hline $47.2-14-1,145$ & 42.27 & 0.08 & 96.12 & $52.0-2-6,145$ & & 0.10 & 0.52 \\
\hline $47.2-14-2,145$ & 42.50 & 0.04 & 96.65 & $52.0-3-2,19$ & & 0.05 & 0.23 \\
\hline $47.2-14-3,145$ & 42.38 & 0.10 & 96.37 & $51.0-3-4,16$ & & 0.08 & 0.07 \\
\hline $47.2-14-5,145$ & 42.59 & 0.05 & 96.85 & $52.0-3-6,6$ & & 0.08 & 0.23 \\
\hline $48.0-2-6,16$ & & 0.40 & 96.47 & $52.0-4-3,5$ & 0.05 & 0.04 & 0.11 \\
\hline $48.0-3-2,28$ & 0.0 & 0.07 & 96.34 & $52.0-4-5,6$ & & 0.21 & 0.23 \\
\hline $48.1-1-1,26$ & 33.15 & 0.03 & 75.38 & $52.0-4-6,128$ & & 0.12 & 0.11 \\
\hline 48.2-1-CC & 40.58 & 0.15 & 92.28 & $52.0-5-2,17$ & 0.05 & 0.02 & 0.11 \\
\hline $48.2-1-1,28$ & 28.20 & 0.12 & 86.87 & $52.0-5-3,15$ & & 0.05 & 0.30 \\
\hline $48.2-1-3,16$ & 36.17 & 0.05 & 82.25 & $52.0-5-4,18$ & 0.05 & 0.02 & 0.11 \\
\hline $48.2-1-5,14.5$ & 37.41 & 0.06 & 85.07 & $52.0-6-1,24$ & & 0.34 & 0.23 \\
\hline $48.2-2-\mathrm{CC}$ & 42.50 & 0.11 & 96.65 & $52.0-6-\mathrm{CC}$ & 0.11 & 0.05 & 0.25 \\
\hline $48.2-2-1,145$ & 42.23 & 0.06 & 96.03 & $52.0-7-\mathrm{CC}$ & 0.05 & 0.05 & 0.11 \\
\hline $48.2-2-2,17$ & 42.60 & 0.05 & 96.87 & $52.0-8-1,145$ & & 0.05 & 0.36 \\
\hline $48.2-2-4,18$ & 42.85 & 0.05 & 97.44 & $52.0-8-2,145$ & & 0.11 & 0.39 \\
\hline $48.2-3-4,17$ & 42.42 & 0.05 & 96.46 & $52.0-8-3,21$ & & 0.10 & 0.57 \\
\hline $49.0-1-1,76$ & 17.90 & 0.04 & 40.70 & $52.0-8-4,145$ & & 0.07 & 0.16 \\
\hline $49.1-1-4,117$ & 25.90 & 0.05 & 58.44 & $52.0-8-5,145$ & 0.14 & Nil & 0.32 \\
\hline $49.1-1-5,60$ & 3.40 & 0.09 & 7.73 & $53.0-1-2,144$ & & 0.05 & 1.36 \\
\hline $49.1-2-1,145$ & 39.72 & 0.09 & 90.32 & $53.0-2-\mathrm{CC}$ & & 0.02 & 2.27 \\
\hline $49.1-2-3,145$ & 35.60 & 0.09 & 80.95 & $53.0-3-1,17$ & & 0.05 & 0.27 \\
\hline $50.0-1-3,340$ & 40.84 & 0.11 & 92.87 & $53.0-4-2$ & 0.07 & 0.09 & 0.16 \\
\hline $50.0-2-1,145$ & 34.71 & 0.08 & 78.93 & $53.0-6-2,65$ & & 0.05 & 82.14 \\
\hline $50.0-2-2,150$ & 34.21 & 0.16 & 77.79 & $53.0-6-\mathrm{CC}$ & 28.42 & 0.28 & 64.62 \\
\hline $50.0-2-3,150$ & 0.62 & 0.03 & 1.41 & $53.0-8-\mathrm{CC}$ & 1.13 & 0.04 & 2.57 \\
\hline $50.0-2-4.150$ & 28.93 & 0.04 & 65.79 & $53.1-1-3,18$ & & 0.10 & 0.64 \\
\hline $50.0-2-6,150$ & 22.40 & 0.05 & 50.94 & $53.1-1-2,7$ & & 0.11 & 0.91 \\
\hline $50.0-2-\mathrm{CC}$ & & 0.09 & Nil & $53.1-1-5,18$ & & 0.09 & 0.08 \\
\hline $50.1-1-3,7$ & 40.13 & 0.05 & 23.04 & $53.1-2-1,145$ & & 0.08 & 2.16 \\
\hline $50.1-1-5,17$ & 15.45 & 0.09 & 35.13 & $53.1-2-2,145$ & 0.96 & 0.05 & 2.16 \\
\hline $50.1-2-1,130$ & 9.39 & 0.04 & 21.35 & $53.1-2-3,90$ & & 0.03 & Nil \\
\hline $50.1-2-5,8$ & 0.04 & 0.02 & 0.09 & $53.1-2-5,7$ & & 0.05 & 0.23 \\
\hline $50.1-3-1,145$ & 0.12 & 0.04 & 0.27 & $53.1-3-1,150$ & 0.46 & 0.08 & 1.05 \\
\hline $50.1-3-5,28$ & & 0.05 & $\mathrm{Nil}$ & $53.1-3-2,40$ & 0.17 & 0.03 & 0.39 \\
\hline $51.0-1-3,6$ & 0.02 & 0.02 & 0.05 & $53.1-3-4,26$ & & 0.18 & Nil \\
\hline $51.0-1-5,15$ & & 0.11 & 0.23 & $53.2-1-2,16$ & & 0.04 & 7.00 \\
\hline $51.0-10-1,175$ & & 0.17 & 1.81 & $53.2-1-6,7$ & & 0.02 & 0.61 \\
\hline $51.1-1-3,30$ & & 0.14 & & $53.2-1-4,7$ & & 0.06 & 1.52 \\
\hline $51.1-1-5,16$ & 0.04 & 0.09 & 0.09 & $54.0-1-1,5$ & 34.90 & 0.08 & 79.36 \\
\hline $51.1-2-1,127$ & & 0.06 & 0.11 & $54.0-1-2,7$ & & 0.02 & 6.41 \\
\hline
\end{tabular}


TABLE 9 - Continued

\begin{tabular}{|c|c|c|c|c|c|c|c|}
\hline Sample Designation & $\begin{array}{l}\mathrm{CO}_{2} \\
(\%)\end{array}$ & $\begin{array}{c}\text { Org. C } \\
(\%)\end{array}$ & $\begin{array}{c}\mathrm{CaCO}_{3} \\
(\%)\end{array}$ & Sample Designation & $\begin{array}{l}\mathrm{CO}_{2} \\
(\%)\end{array}$ & $\begin{array}{c}\text { Org. C } \\
(\%)\end{array}$ & $\begin{array}{c}\mathrm{CaCO}_{3} \\
(\%)\end{array}$ \\
\hline $54.0-1-2,20$ & & Nil & 5.93 & $56.2-1-2,145$ & & 0.09 & 95.68 \\
\hline $54.0-2-1,17$ & & Nil & 4.11 & $56.2-2-4,36$ & & 0.14 & 95.09 \\
\hline $54,0-2-4,16$ & & 0.03 & 8.66 & $56.2-3-2,145$ & & 0.07 & 96.02 \\
\hline $54.0-3-1,140$ & 5.06 & 0.10 & 11.51 & $56.2-3-6,145$ & & 0.06 & 94.16 \\
\hline $54.0-4-2,14$ & & 0.05 & 11.30 & $56.2-4-4,14$ & & 0.17 & 93.18 \\
\hline $54.0-6-1,145$ & 0.60 & 0.04 & 1.36 & $56.2-5-2,13$ & & 0.08 & 91.93 \\
\hline $54.0-6-3,23$ & & 0.08 & 10.30 & $56.2-5-6,7$ & & 0.10 & 92.56 \\
\hline $54.0-6-4,78$ & & 0.06 & 8.25 & $56.2-6-2,7$ & & 0.07 & 90.56 \\
\hline $54.0-7-1,138$ & & 0.05 & 2.41 & $56.2-7-6,7$ & & 0.05 & 93.13 \\
\hline $54.0-7-3,16$ & & Nil & 1.84 & $56.2-8-4,7$ & & 0.03 & 92.79 \\
\hline $55.0-1-2,73$ & & 0.22 & 87.74 & $56.2-9-4,19$ & & 0.03 & 87.10 \\
\hline $55.0-1-2,30$ & 38.53 & 0.25 & 87.62 & $56.2-10-1,10$ & & 0.04 & 85.74 \\
\hline $55.0-1-4,7$ & & 0.16 & 88.81 & $57.1-1-1,10$ & & & 93.04 \\
\hline $55.0-1-6,7$ & & 0.12 & 87.90 & $57.1-1-2,13$ & & 0.03 & 95.57 \\
\hline $55.0-2-2,7$ & & 0.24 & 91.70 & $57.1-1-4,7$ & & 0.13 & 93.29 \\
\hline $55.0-2-4,15$ & & 0.22 & 91.81 & $57.1-1-6,11$ & & 0.06 & 93.01 \\
\hline $55.0-2-5,70$ & & 0.10 & 93.20 & $57.1-3-1,17$ & 37.01 & 0.12 & 84.16 \\
\hline $55.0-3-1,16$ & & 0.12 & 92.68 & $57.1-3-4,22$ & & 0.03 & 85.17 \\
\hline $55.0-3-4,145$ & & 0.06 & 94.63 & $57.1-4-1,26$ & & 0.05 & 68.68 \\
\hline $55.0-4-1,13$ & & 0.06 & 94.47 & $57.1-43,14$ & & 0.03 & 78.76 \\
\hline $55.0-4-3,7$ & & 0.05 & 95.00 & $57.1-4-4,145$ & 33.51 & 0.15 & 76.20 \\
\hline $55.0-4-5,7$ & & 0.10 & 94.02 & $58.1-1-1,139$ & & 0.17 & 48.83 \\
\hline $55.0-5-7-1,7$ & & 0.07 & 93.40 & $58.1-1-2,91$ & & 0.17 & 90.97 \\
\hline $55.0-5-5,7$ & & 0.05 & 93.04 & $58.2-1-2,16$ & & 0.08 & 64.16 \\
\hline $55.0-6-3,17$ & & 0.05 & 92.02 & $58.2-1-4,31$ & & 0.07 & 36.23 \\
\hline $55.0-7-1,14$ & & 0.06 & 90.97 & $59.1-3-\mathrm{CC}$ & 0.05 & 0.16 & 0.11 \\
\hline $55.0-7-5,19$ & & 0.07 & 91.38 & $59.2-3 \mathrm{CC}$ & 0.05 & 0.05 & 0.11 \\
\hline $55.0-8-3,16$ & & 0.05 & 91.79 & $59.2-5-\mathrm{CC}$ & 0.20 & 0.05 & 0.45 \\
\hline $55.0-9$, Top & 38.30 & 0.14 & 87.05 & 59.2-6, Тop & 0.20 & 0.10 & 0.45 \\
\hline $55.0-10-2,10$ & & 0.06 & 87.05 & $60.0-1-\mathrm{CC}$ & 13.88 & 0.15 & 31.56 \\
\hline $55.0-10-2,10$ & & 0.06 & 79.82 & $60.0-3-\mathrm{CC}$ & 2.94 & 0.10 & 6.69 \\
\hline $55.0-10-5,16$ & & 0.03 & 85.42 & $60.0-4-\mathrm{CC}$ & 1.12 & 0.05 & 2.55 \\
\hline $55.0-14-2,145$ & & 0.05 & 93.50 & $60.0-4-1,145$ & 3.38 & Nil & 7.69 \\
\hline $55.0-14-4,145$ & & 0.05 & 93.54 & $60.0-4-1,145$ & 3.41 & Nil & 7.75 \\
\hline $55.0-14-6,145$ & & 0.11 & 93.95 & $60.0-4-2,145$ & 0.90 & 0.03 & 2.05 \\
\hline $56.2-1-6,7$ & & 0.07 & 96.07 & $60.0-5-\mathrm{CC}$ & 2.00 & 0.04 & 4.55 \\
\hline
\end{tabular}


TABLE 10

Results of Determination of Phosphorous

\begin{tabular}{|c|c|c|c|}
\hline Sample Designation & $\mathrm{P}_{2} \mathrm{O}_{5} \%$ & Sample Designation & $\mathrm{P}_{2} \mathrm{O}_{5} \%$ \\
\hline $44.0-1, \mathrm{CC}$ & 0.39 & $47.2-13-\mathrm{CC}$ & 0.02 \\
\hline $44.0-1-1,145$ & 0.07 & $47.2-13-5,145$ & 0.06 \\
\hline $44.0-1-2,145$ & 0.07 & $47.2-14-\mathrm{CC}$ & 0.02 \\
\hline $44.0-1-3,145$ & 0.08 & $47.2-14-1,145$ & 0.06 \\
\hline $44.0-2-\mathrm{CC}$ & 0.10 & $47.2-14-3,145$ & 0.03 \\
\hline $44.0-2-5,145$ & 0.06 & $48.2-1-1,28$ & 0.07 \\
\hline $44.0-2-6,145$ & 0.07 & $48.2-2-\mathrm{CC}$ & 0.02 \\
\hline $44.0-3-\mathrm{CC}$ & 0.08 & $48.2-2-1,145$ & 0.08 \\
\hline $44.0-3-\mathrm{CC}$ & 0.08 & $48.2-11-\mathrm{CC}$ & 0.08 \\
\hline $44.0-3-2,145$ & 0.09 & $49.1-1-5,60$ & 0.17 \\
\hline $44.0-3-3,145$ & 0.08 & $50.0-2-1,145$ & 0.14 \\
\hline $44.0-3-4,145$ & 0.10 & $50.0-2-2,150$ & 0.15 \\
\hline $44.0-3-5,145$ & 0.08 & $50.0-2-3,150$ & 0.16 \\
\hline $44.0-4-C C$ & 0.20 & $50.0-2-4,150$ & 0.13 \\
\hline 44.0-4-0, Top & 0.07 & $50.0-2-6,150$ & 0.11 \\
\hline $44.0-4-2,145$ & 0.08 & $51.0-1-\mathrm{CC}$ & 0.55 \\
\hline $44.0-4-3,145$ & 0.08 & $52.0-2-\mathrm{CC}$ & 0.36 \\
\hline $44.0-4-4,145$ & 0.11 & $52.0-6-\mathrm{CC}$ & 0.40 \\
\hline $44.0-4-4,145$ & 0.09 & $52.0-9-\mathrm{CC}$ & 1.27 \\
\hline $44.0-4-5,145$ & 0.11 & $52.0-10-\mathrm{CC}$ & 0.57 \\
\hline $44.0-4-6,145$ & 0.09 & $53.0-2-\mathrm{CC}$ & 0.17 \\
\hline $44.0-5-$ & 0.12 & $53.0-4-2$ & 0.10 \\
\hline $47.0-1-4,95$ & 0.15 & $53.0-6-\mathrm{CC}$ & 0.07 \\
\hline 47.1-1, Top & 0.18 & $54.0-1-1,5$ & 0.16 \\
\hline $47.2-3-\mathrm{CC}$ & 0.04 & $53.1-2-2,145$ & 0.12 \\
\hline $47.2-4-1-\mathrm{CC}$ & 0.09 & $54.0-1-2,20$ & 0.17 \\
\hline $47.2-8-6-\mathrm{CC}$ & 0.14 & $54.0-3-1,140$ & 0.13 \\
\hline $47.2-9-\mathrm{CC}$ & 0.46 & $54.0-6-1,145$ & 0.14 \\
\hline $47.2-9-1,150$ & 0.17 & $55.0-3-4,145$ & 0.09 \\
\hline $47.2-9-4,0$ & 0.10 & $55.0-9$, Тор & 0.08 \\
\hline $47.2-9-5,150$ & 0.13 & $55.0-13-3,40$ & 0.04 \\
\hline $47.2-9-6,0$ & 0.19 & $57.1-4,145$ & 0.08 \\
\hline $47.2-10-\mathrm{CC}$ & 0.24 & $59.2-5-\mathrm{CC}$ & 1.05 \\
\hline 47.2-10, Top & 0.14 & 59.2-6, Top & 1.69 \\
\hline $47.2-11-\mathrm{CC}$ & 0.06 & $60.0-3-\mathrm{CC}$ & 0.21 \\
\hline $47.2-11-3,145$ & 0.13 & $60.0-4-\mathrm{CC}$ & 0.22 \\
\hline $47.2-11-4,145$ & 0.07 & $60.0-4-1,145$ & 0.16 \\
\hline $47.2-11-6,145$ & 0.05 & $60.0-4-2,145$ & 0.22 \\
\hline $47.2-12-\mathrm{CC}$ & 0.03 & $60.0-5-\mathrm{CC}$ & 0.17 \\
\hline $47.2-12-1,145$ & 0.03 & & \\
\hline
\end{tabular}


TABLE 11

Spectrochemical Analysis of Trace Elements

\begin{tabular}{|c|c|c|c|c|c|c|c|c|c|c|}
\hline Sample Designation & $\mathrm{Mn}$ & $\mathrm{Ti}$ & $\mathrm{Cr}_{\mathrm{r}}$ & V & $\mathrm{Ni}$ & Co & $\mathrm{Zr}$ & $\mathrm{Cu}$ & $\mathrm{Pb}$ & Mo \\
\hline $6-44.0-1 \cdot 1,145$ & $<500$ & $<300$ & $<10$ & $<5$ & $<5$ & $<5$ & 5.2 & 21 & 20 & $<5$ \\
\hline $6-44 \cdot 0 \cdot 1 \cdot 2,145$ & 320 & $<300$ & $<10$ & $<5$ & $<5$ & $<5$ & $<2$ & 10 & 17 & $<5$ \\
\hline $6-44.0-1-3,50$ & 310 & $<300$ & $<10$ & $<5$ & $<5$ & $<5$ & 3.6 & 420 & $<5$ & $<5$ \\
\hline $6-44.0-1-3,145$ & $<300$ & $<300$ & $<10$ & $<5$ & $<5$ & $<5$ & 3.6 & 20 & 16 & $<5$ \\
\hline 6-44.0-1-3, mixed & 360 & $<300$ & $<10$ & $<5$ & $<5$ & $<5$ & $<2$ & 20 & 10 & $<5$ \\
\hline $6-44.0-1-4,145$ & 360 & $<300$ & $<10$ & $<5$ & $<5$ & $<5$ & 3.6 & 19 & 26 & $<5$ \\
\hline 6-44.0-1, CC & 24,000 & 7,000 & 290 & 150 & 530 & 175 & 190 & $<500$ & 27 & 76 \\
\hline $6-44.0-2-1,145$ & 430 & $<300$ & $<10$ & $<5$ & $<5$ & $<5$ & 5.6 & 15 & $<5$ & $<5$ \\
\hline $6-44 \cdot 0-2-2,145$ & 350 & $<300$ & $<10$ & $<5$ & $<5$ & $<5$ & $<2$ & 10 & $<5$ & $<5$ \\
\hline $6-44.0-2-3,145$ & 300 & $<300$ & $<10$ & $<5$ & $<5$ & $<5$ & 2.4 & 17 & $<5$ & $<5$ \\
\hline $6-44.0-2-4,145$ & 1,350 & 330 & $<10$ & 7 & 13 & - & 6.6 & 84 & 10 & $<5$ \\
\hline $6-44.0-2-5,145$ & 530 & $<300$ & $<10$ & $<5$ & 7 & - & 5 & 12 & 11.5 & $<5$ \\
\hline $6-44 \cdot 0-2-6,145$ & 470 & $<300$ & $<10$ & $<5$ & $<5$ & - & 4.6 & 17.5 & 17 & $<5$ \\
\hline $6-44.0-2, \mathrm{CC}$ & 530 & $<100$ & $<10$ & $<5$ & $<5$ & $<5$ & 2 & 10 & 6 & $<5$ \\
\hline $6-44.0-3-2,145$ & 820 & $<300$ & $<10$ & $<5$ & 12 & - & 4 & 16.5 & 13 & $<5$ \\
\hline $6-44.0-3-3,145$ & 380 & $<300$ & $<10$ & $<5$ & 11 & - & 4.6 & $<10$ & $<5$ & $<5$ \\
\hline $6-44.0-3-4,145$ & 520 & $<300$ & $<10$ & $<5$ & 12 & - & 4 & 39 & $<5$ & $<5$ \\
\hline $6-44.0-3-5,145$ & 820 & $<300$ & $<10$ & $<5$ & 11 & - & 5.8 & 500 & $<5$ & $<5$ \\
\hline $6-44.0-3, \mathrm{CC}$ & 890 & $<300$ & $<10$ & $<5$ & 11 & $<5$ & - & - & 20 & $<5$ \\
\hline $6-44.0-4$, top & 660 & $<300$ & $<10$ & $<5$ & 16 & - & $<2$ & 13.5 & $<5$ & 18 \\
\hline $6-44.0-4-0$, top & $<500$ & $<300$ & $<10$ & $<5$ & $<5$ & $<5$ & 7 & - & $<5$ & $<5$ \\
\hline $6-44.0-4-2,145$ & 660 & $<300$ & $<10$ & $<5$ & 14 & - & 4.6 & 145 & $<5$ & 10 \\
\hline $6-44.0-4-3,145$ & 820 & $<300$ & $<10$ & $<5$ & 13 & - & 4.3 & $<10$ & $<5$ & $<5$ \\
\hline $6-44.0-4-4,145$ & 880 & $<300$ & $<10$ & $<5$ & 16 & - & 3.5 & $<10$ & 14 & $<5$ \\
\hline $6-44.0-4-5,145$ & 550 & $<300$ & $<10$ & $<5$ & 11 & 5 & $<2$ & 20 & 6 & $<5$ \\
\hline $6-44.0-4-6,145$ & 720 & $<300$ & $<10$ & $<5$ & 10 & $<5$ & 2.4 & 18 & $<5$ & $<5$ \\
\hline $6-44.0-4, \mathrm{CC}$ & $<510$ & $<100$ & $<10$ & $<5$ & 10 & $<5$ & 3.2 & $<10$ & $<5$ & $<5$ \\
\hline $6-44.0-5$, top & 750 & $<300$ & 15 & $<5$ & 10 & $<5$ & $<2$ & $<500$ & 54 & 66 \\
\hline $6-45 \cdot 1-1-1,7$ & 9,000 & 10,000 & 64 & 140 & 390 & 230 & 420 & 210 & $6 ?$ & 62 \\
\hline $6-45.1-1-1,70$ & 20,000 & 8,000 & 113 & 160 & 600 & 195 & 150 & 190 & 98 & 26 \\
\hline $6-45.1-1-1,145$ & 8,000 & 12,600 & 115 & 260 & 460 & 180 & 330 & 190 & 7 & 80 \\
\hline $6-45.1-1-2,100$ & 10,000 & 13,000 & 118 & 290 & 550 & 200 & 390 & 280 & 35 & 88 \\
\hline $6-45.1-1-2,124$ & 2,400 & 15,500 & 460 & 220 & 300 & 66 & 145 & 160 & $<5$ & $<5$ \\
\hline $6-45.1-1-3,23$ & 1,500 & 18,500 & 440 & 220 & 310 & 62 & 170 & 110 & $<5$ & $<5$ \\
\hline $6-45.1-1-4,22$ & 2,400 & 15,000 & 490 & 220 & 300 & 64 & 135 & 152 & $<5$ & $?$ \\
\hline $6-45.1-1-4,136$ & 1,950 & 8,900 & 270 & 108 & 370 & 41 & 89 & 105 & $<5$ & $<5$ \\
\hline $6-45.1-1-5,4$ & 3,000 & 12,000 & 490 & 180 & 530 & 66 & 105 & 124 & $<5$ & $<5$ \\
\hline $6-45.1-1, \mathrm{CC}$ & 7,000 & 2,100 & 14 & 40 & 200 & 30 & 90 & 210 & $<5$ & 5 \\
\hline $6-45.1 \cdot 3, \mathrm{CC}$ & $<500$ & 700 & 12 & 14.5 & 20 & $<5$ & 22 & - & 6 & $<5$ \\
\hline
\end{tabular}


TABLE 11 - Continued

\begin{tabular}{|c|c|c|c|c|c|c|c|c|c|c|}
\hline Sample Designation & $\mathrm{Mn}$ & $\mathrm{Ti}$ & $\mathrm{Cr}$ & V & $\mathrm{Ni}$ & Co & $\mathrm{Zr}$ & $\mathrm{Cu}$ & $\mathrm{Pb}$ & Mo \\
\hline $6-45.1 \cdot 3, \mathrm{CC}$ & 1,180 & 1,220 & 14 & 25 & 41 & 9 & 50 & - & 8 & $<25$ \\
\hline $6-46.0$ & $<500$ & 860 & $<10$ & 22 & 8 & $<5$ & 26 & 117 & $<5$ & $<5$ \\
\hline $6-46.0-1-1,145$ & 7,500 & 3,800 & 77 & 110 & 200 & 89 & 260 & 220 & 32 & 50 \\
\hline $6-46.0-1-2,145$ & 8,600 & 4,400 & 77 & 200 & 240 & 89 & 390 & 310 & 72 & 36 \\
\hline $6-46.0-1-3,145$ & 7,500 & 3,300 & 66 & 90 & 165 & 76 & 165 & 240 & 60 & 25 \\
\hline $6-46.0-1-4,145$ & 23,000 & 3,400 & 50 & 105 & 290 & 110 & 210 & 280 & 100 & 50 \\
\hline $6-46.0-1-5,145$ & 3,700 & 2,600 & 38 & 60 & 110 & 48 & 80 & 240 & 55 & 25 \\
\hline $6-46.0-1-6$ & $<500$ & 690 & $<10$ & 9.5 & 7 & $<5$ & 24 & 130 & $<5$ & $<5$ \\
\hline $6-46.0-1-6$ & 300 & 1,470 & 14 & 29 & 21 & $<5$ & 40 & 170 & 6 & $<5$ \\
\hline $6-46.0-1-6,145$ & 6,100 & 6,700 & 58 & 180 & 195 & 105 & 280 & 120 & 15 & 58 \\
\hline $6-46.1-2, \mathrm{CC}$ & 5,700 & 2,200 & 16 & 39 & 250 & 26 & 110 & 280 & 23 & 5 \\
\hline $6-47.0-1-1,17$ & $<500$ & 3,400 & 32 & 68 & 42 & 12 & 105 & - & $<10$ & $<5$ \\
\hline $6-47.0-1-2,5$ & 1,300 & 3,500 & 30 & 165 & 38 & 15.5 & 310 & 80 & 20 & $<5$ \\
\hline $6-47.0-1-3,16$ & 660 & 2,050 & 20 & 94 & 46 & 12 & 105 & - & 16 & $<5$ \\
\hline $6-47.0-1-4,90$ & $<500$ & 2,600 & 32 & 62 & 33 & 11 & 50 & - & 210 & $<5$ \\
\hline $6-47.0-1-5,3.5$ & 560 & 1,130 & 12 & 33 & 10 & $<5$ & 48 & - & 14 & $<5$ \\
\hline $6-47.0-1 \cdot 6,14$ & 2,500 & 1,530 & 15 & 34 & 18 & 8 & 31 & - & $<10$ & $<5$ \\
\hline $6-47.1-1-1,145$ & 880 & 490 & $<10$ & 15 & 18.5 & 10 & 16.5 & $<10$ & 18.5 & $<10$ \\
\hline $6-47.1-1-2,16$ & 850 & $<300$ & $<10$ & 5 & 7 & $<5$ & $<5$ & - & $<10$ & $<5$ \\
\hline $6-47.2-1-2,23$ & $<500$ & 1,920 & 18 & 47 & 21 & 6 & 28 & - & $<10$ & $<5$ \\
\hline $6-47 \cdot 2-1-3,11$ & 1,540 & 1,820 & 19.5 & 32 & 23 & 10 & 57 & 31 & 14 & $<5$ \\
\hline $6-47.2-1-3,90$ & $<500$ & 1,440 & $<10$ & 45 & 15 & $<10$ & 32 & $<10$ & $<10$ & $<10$ \\
\hline $6-47.2-1-4,7$ & 780 & 1,500 & $<10$ & 36 & 18 & 5.7 & 35 & 23 & 10 & $<5$ \\
\hline $6-47.2-2-2,47$ & 830 & 1,700 & 27 & 57 & 33 & 9.4 & 72 & 43 & 24 & $<5$ \\
\hline $6-47 \cdot 2-2-3,7$ & 1.400 & 630 & $<10$ & 13 & 6 & $<5$ & 18 & 15 & $<5$ & $<5$ \\
\hline $6-47.2-2-4,7$ & - & $<500$ & 1,500 & 10 & 32 & 14 & $<5$ & 32 & $<10$ & $<5$ \\
\hline $6-47.2-2-6,6$ & - & $<500$ & 680 & 10 & 1.7 & 14 & $<5$ & 14 & $<10$ & $<5$ \\
\hline $6-47.2-3-2,65$ & 700 & 1,650 & 12.5 & 52 & 40 & 17 & 34 & 30 & 6 & $<5$ \\
\hline $6-47 \cdot 2-3-3,6$ & $<500$ & 800 & 10 & 15 & 14 & $<5$ & 25 & - & $<10$ & $<5$ \\
\hline $6-47.2-3-4,16$ & 890 & 1,200 & $<10$ & 37 & 5 & $<5$ & 19.5 & $<10$ & $<5$ & $<5$ \\
\hline $6-47.2-3, \mathrm{CC}$ & $<500$ & 420 & $<10$ & 14.5 & 8 & 5 & 13 & $<10$ & $<5$ & $<5$ \\
\hline $6-47.2-4-1,16$ & 550 & 430 & $<10$ & 8 & 5 & $<5$ & 8 & - & $<10$ & $<5$ \\
\hline $6-47.2-4-2,17$ & 1,150 & 340 & $<10$ & 15.5 & 7 & $<5$ & 9.0 & 36 & $<5$ & $<5$ \\
\hline $6-47.2-4-3,16$ & $<500$ & 370 & $<10$ & 10 & 7 & $<5$ & 7 & - & $<10$ & $<5$ \\
\hline $6-47.2-4-4,16$ & 830 & 1,150 & $<10$ & 37 & 8 & $<5$ & 19.5 & $<10$ & $<5$ & $<5$ \\
\hline $6-47.2-4-5,16$ & 350 & 510 & $<10$ & 12 & 11 & $<5$ & 13 & - & $<10$ & $<5$ \\
\hline $6-47.2-4-6,17$ & 1,350 & 560 & $<10$ & 17 & 6 & $<5$ & 14.7 & $<10$ & $<5$ & $<5$ \\
\hline $6-47.2-4, \mathrm{CC}$ & 800 & 700 & $<10$ & 15 & 11 & $<5$ & 12.5 & 41 & 26 & $<5$ \\
\hline $6-47.2-5-1,17$ & 520 & 460 & $<10$ & 12 & 11 & 50 & 11 & - & $<10$ & $<5$ \\
\hline $6-47.2-5-2,6$ & 1,250 & 590 & $<10$ & 14 & 7 & 7 & 9.6 & 34 & 15 & $<5$ \\
\hline
\end{tabular}


TABLE 11 - Continued

\begin{tabular}{|c|c|c|c|c|c|c|c|c|c|c|}
\hline Sample Designation & $\mathrm{Mn}$ & $\mathrm{Ti}$ & $\mathrm{Cr}$ & V & $\mathrm{Ni}$ & Co & $\mathrm{Zr}$ & $\mathrm{Cu}$ & $\mathrm{Pb}$ & Mo \\
\hline $6-47 \cdot 2-5 \cdot 3,6$ & 640 & 480 & $<10$ & 10 & 11 & $<5$ & 12 & - & $<10$ & $<5$ \\
\hline $6-47 \cdot 2-5-4,6$ & 1,350 & 1,400 & $<10$ & 42 & 19.5 & 17.5 & $<10$ & 21 & $<5$ & $<5$ \\
\hline $6-47.2-5-5,6$ & 820 & 580 & $<10$ & 13 & 7 & $<5$ & 20 & - & $<10$ & $<5$ \\
\hline $6-47.2-5-6,65$ & 1,400 & 580 & $<10$ & 9 & 12.5 & 4.5 & 9 & 18 & 12 & $<5$ \\
\hline $6-47.2-6-2,6$ & 1,480 & 870 & $<10$ & 16 & 14 & $<5$ & 24 & - & $<10$ & $<5$ \\
\hline $6-47.2-6-3,17$ & 1,950 & 760 & $<10$ & 20 & 17.5 & 9 & 14.7 & 48 & 7 & $<5$ \\
\hline $6-47 \cdot 2-7-1,17$ & 1,330 & 880 & $<10$ & 17 & 39 & 21 & 17 & - & $<10$ & $<5$ \\
\hline $6-47 \cdot 2-7-2,17$ & 1,020 & $<300$ & $<10$ & $<5$ & $<5$ & $<5$ & $<5$ & $100 ?$ & $<5$ & $<5$ \\
\hline $6-47.2-7-3,13$ & 720 & $<300$ & $<10$ & $<5$ & $<5$ & $<5$ & $<5$ & - & $<10$ & $<5$ \\
\hline $6-47.2-7-4,17$ & 1,350 & $<300$ & $<10$ & 8 & $<5$ & $<5$ & 6 & 70 & $<5$ & $<5$ \\
\hline $6-47.2-7-5,27$ & 900 & $<300$ & $<10$ & 6 & $<5$ & $<5$ & $<5$ & - & $<10$ & $<5$ \\
\hline $6-47.2-7-6,126$ & 850 & $<300$ & $<10$ & $<5$ & $<5$ & $<5$ & $<5$ & $<10$ & $<5$ & $<5$ \\
\hline $6-47.2-8-1,27$ & $<500$ & $<300$ & $<10$ & 6 & $<5$ & $<5$ & $<5$ & - & $<10$ & $<5$ \\
\hline $6-47.2-8-2,17$ & 550 & $<300$ & $<10$ & $<5$ & $<5$ & $<5$ & $<5$ & 11.5 & $<5$ & $<5$ \\
\hline $6-47.2-8-3,18$ & $<500$ & $<300$ & $<10$ & $<5$ & $<5$ & $<5$ & $<5$ & - & $<10$ & $<5$ \\
\hline $6-47.2-8-5,17$ & $<500$ & $<300$ & $<10$ & $<10$ & $<10$ & $<10$ & $<5$ & 10 & $<10$ & $<10$ \\
\hline $6-47 \cdot 2-8-5,5$ & 1,100 & 1,100 & $<10$ & 22 & 9 & $<5$ & 13.5 & 33 & 6 & $<5$ \\
\hline $6-47.2-8-6,16$ & 810 & $<300$ & $<10$ & $<5$ & $<5$ & 7.4 & $<5$ & 13.5 & $<5$ & $<5$ \\
\hline $6-47.2-8-6, \mathrm{CC}$ & 540 & $<300$ & $<10$ & 5 & 0 & $<5$ & 5.8 & $<10$ & 18 & 32 \\
\hline $6-47.2-9-1,16$ & $<500$ & $<300$ & $<10$ & $<10$ & $<10$ & $<10$ & $<5$ & $<10$ & $<10$ & $<10$ \\
\hline $6-47.2-9-1,150$ & 670 & $<300$ & $<10$ & $<5$ & $<5$ & $<5$ & $<2$ & 47 & $<5$ & $<5$ \\
\hline $6-47.2-9-2,17$ & 760 & $<300$ & $<10$ & 5 & 9 & 8.6 & 5 & $<10$ & $<5$ & $<5$ \\
\hline $6-47.2-9-3,17$ & $<500$ & $<300$ & $<10$ & 5 & 7 & 5 & $<5$ & - & $<10$ & $<5$ \\
\hline $6-47.2-9-4,0$ & 640 & 150 & $<10$ & 6 & 5 & $<5$ & 3 & 17.5 & 7 & $<5$ \\
\hline $6-47.2-9-5,17$ & 1,230 & $<300$ & $<10$ & 7 & 10 & 6.8 & 16 & 27 & $<5$ & $<5$ \\
\hline $6-47.2-9-5,150$ & 1,050 & $<300$ & $<10$ & $<5$ & $<5$ & $<5$ & $<2$ & 35 & $<5$ & $<5$ \\
\hline $6-47.2-9-6,0$ & 700 & 760 & $<10$ & 14 & 15 & - & 23 & 500 & 13 & $<5$ \\
\hline $6-47.2-9, \mathrm{CC}$ & 1,200 & 310 & $<10$ & 7.6 & 12 & 7 & 7.2 & 19 & 6 & $<5$ \\
\hline $6-47.2-10$, top & $<500$ & $<300$ & $<10$ & $<5$ & $<5$ & $<5$ & $<2$ & 20 & $<5$ & $<5$ \\
\hline $6-47.2 \cdot 10-1,17$ & $<500$ & $<300$ & $<10$ & $<5$ & $<5$ & $<5$ & $<5$ & $<10$ & $<5$ & $<5$ \\
\hline $6-47 \cdot 2-10-2,17$ & $<500$ & $<300$ & $<10$ & $<10$ & $<10$ & $<10$ & $<5$ & 10 & $<10$ & $<10$ \\
\hline $6-47.2-10-3,18$ & 740 & $<300$ & 40 & $<5$ & $<5$ & $<5$ & $<5$ & 16 & $<5$ & $<5$ \\
\hline $6-47.2-10-4,17$ & $<500$ & $<300$ & $<10$ & $<10$ & 20 & $<10$ & 11 & $<10$ & $<10$ & $<10$ \\
\hline $6-47.2-10-5,17$ & 3,300 & $<300$ & $<10$ & 7 & 15 & 13 & 16 & 23 & $<5$ & $<5$ \\
\hline $6-47.2-10-6,16$ & $<500$ & $<300$ & $<10$ & $<10$ & $<10$ & $<10$ & $<5$ & $<10$ & $<10$ & $<10$ \\
\hline $6-47.2-10, \mathrm{CC}$ & 650 & 170 & $<10$ & 6 & $<5$ & $<5$ & 2 & $<10$ & 5 & $<5$ \\
\hline $6-47.2-11-1,145$ & 500 & $<300$ & $<10$ & $<5$ & $<5$ & $<5$ & $<5$ & 18.5 & $<5$ & $<5$ \\
\hline $6-47.2-11-2,145$ & $<500$ & $<300$ & $<10$ & $<5$ & 5 & $<5$ & $<5$ & - & $<10$ & $<5$ \\
\hline $6-47.2-11-3,145$ & $<500$ & $<300$ & $<10$ & $<5$ & $<5$ & $<5$ & $<2$ & 24 & $<5$ & $<5$ \\
\hline $6-47.2-11-4,145$ & 400 & $<300$ & $<10$ & $<5$ & $<5$ & $<5$ & 7 & 15 & $<5$ & $<5$ \\
\hline
\end{tabular}


TABLE 11 - Continued

\begin{tabular}{|c|c|c|c|c|c|c|c|c|c|c|}
\hline Sample Designation & $\mathrm{Mn}$ & $\mathrm{Ti}$ & $\mathrm{Cr}$ & V & $\mathrm{Ni}$ & Co & $\mathrm{Zr}$ & $\mathrm{Cu}$ & $\mathrm{Pb}$ & Mo \\
\hline $6-47.2-11-5,145$ & 650 & $<300$ & $<10$ & $<5$ & $<5$ & $<5$ & $<5$ & $<10$ & $<5$ & $<5$ \\
\hline $6-47.2-11-6,145$ & 390 & $<300$ & $<10$ & $<5$ & $<5$ & $<5$ & 7.2 & 76 & 5.5 & $<5$ \\
\hline $6-47.2-11, \mathrm{CC}$ & $<500$ & $<100$ & $<10$ & $<5$ & $<5$ & $<5$ & 2.5 & 10 & 5 & $<5$ \\
\hline $6-47.2-12-1,145$ & $<300$ & $<300$ & $<10$ & $<5$ & $<5$ & $<5$ & 5.2 & 17 & $<5$ & $<5$ \\
\hline $6-47 \cdot 2-12-2,17$ & $<500$ & $<300$ & $<10$ & $<5$ & $<5$ & $<5$ & $<5$ & - & $<10$ & $<5$ \\
\hline $6-47.2-12-3,145$ & $<500$ & $<300$ & $<10$ & $<5$ & $<5$ & $<5$ & $<5$ & 19.5 & $<5$ & $<5$ \\
\hline $6-47.2-12-4,145$ & $<500$ & $<300$ & $<10$ & $<10$ & $<10$ & $<10$ & $<5$ & $<10$ & $<10$ & $<10$ \\
\hline $6-47.2-12, \mathrm{CC}$ & $<500$ & $<100$ & $<10$ & $<5$ & $<5$ & $<5$ & 3.7 & 28 & $<5$ & $<5$ \\
\hline $6-47 \cdot 2-13-1,145$ & $<500$ & $<300$ & $<10$ & $<5$ & $<5$ & $<5$ & $<5$ & 17 & $<5$ & $<5$ \\
\hline $6-47.2-13-2,145$ & $<500$ & $<300$ & $<10$ & $<5$ & $<5$ & $<5$ & $<5$ & - & $<10$ & $<5$ \\
\hline $6-47 \cdot 2-13-3,16$ & 430 & $<300$ & $<10$ & $<5$ & $<5$ & $<5$ & $<5$ & 13.5 & $<5$ & $<5$ \\
\hline $6-47.2-13-4,145$ & 720 & $<300$ & $<10$ & $<5$ & $<5$ & $<5$ & $<5$ & 46 & $<5$ & $<5$ \\
\hline $6-47.2-13-5,145$ & $<500$ & $<300$ & $<10$ & $<5$ & $<5$ & $<5$ & $<2$ & $<10$ & $<5$ & $<5$ \\
\hline $6-47.2-13-6,145$ & $<500$ & $<300$ & $<10$ & $<5$ & $<5$ & $<5$ & $<5$ & - & $<10$ & $<5$ \\
\hline $6-47.2-13, \mathrm{CC}$ & $<500$ & $<300$ & $<10$ & $<5$ & $<5$ & $<5$ & $<2$ & $<10$ & 6 & $<5$ \\
\hline $6-47.2-14-1,145$ & $<500$ & $<300$ & $<10$ & $<5$ & $<5$ & $<5$ & $<5$ & $<10$ & $<5$ & $<5$ \\
\hline $6-47.2-14-2,145$ & $<500$ & $<300$ & $<10$ & $<5$ & $<5$ & $<5$ & $<5$ & 12 & $<5$ & $<5$ \\
\hline $6-47.2-14-3,145$ & $<500$ & $<300$ & $<10$ & $<5$ & $<5$ & $<5$ & $<2$ & 10 & $<5$ & $<5$ \\
\hline $6-47.2-14-4,145$ & $<500$ & $<300$ & $<10$ & $<5$ & $<5$ & $<5$ & $<5$ & - & $<10$ & $<5$ \\
\hline $6-47.2-14-5,145$ & $<500$ & $<300$ & $<10$ & $<5$ & $<5$ & $<5$ & $<5$ & 18.5 & $<5$ & $<5$ \\
\hline $6-47.2-14-6,140$ & $<500$ & $<300$ & $<10$ & $<5$ & $<5$ & $<5$ & $<5$ & - & $<10$ & $<5$ \\
\hline $6-47.2-14, \mathrm{CC}$ & $<500$ & $<100$ & $<10$ & $<5$ & $<5$ & $<5$ & 5.2 & 27 & 6.5 & $<5$ \\
\hline $6-48.1-1-1,26$ & 3,800 & 1,250 & 19.5 & 27 & 54 & 30 & 62 & 85 & 12 & $<5$ \\
\hline $6-48.2-1-1,28$ & 920 & 660 & $<10$ & 14 & 6 & $<5$ & 12 & 13 & 6 & $<5$ \\
\hline $6-48.2-1-2,33$ & $<500$ & 500 & $<10$ & 11 & 18 & 10 & 13 & - & $<10$ & $<5$ \\
\hline $6-48.2-1-3,16$ & 700 & 430 & $<10$ & 13.3 & 19 & 8 & 24 & - & 11 & $<5$ \\
\hline $6-48.2-1-4,16$ & 590 & 480 & $<10$ & 9 & 9 & $<5$ & 11 & - & $<10$ & $<5$ \\
\hline $6-48.2-1-5,14.5$ & 1,270 & 460 & $<10$ & 13.3 & 17 & 8 & 12.5 & - & 11 & $<5$ \\
\hline $6-48.2-1-6,11$ & $<500$ & $<300$ & $<10$ & $<5$ & $<5$ & $<5$ & $<5$ & - & $<10$ & $<5$ \\
\hline $6-48.2-1, \mathrm{CC}$ & 470 & 320 & $<10$ & $<5$ & 76 & $<5$ & 9 & 21 & 5.5 & $<5$ \\
\hline $6-48 \cdot 2-2-1,145$ & $<500$ & $<300$ & $<10$ & $<5$ & $<5$ & $<5$ & $<2$ & 100 & $<5$ & $<5$ \\
\hline $6-48.2-2-2,17$ & $<500$ & $<300$ & $<10$ & $<5$ & $<5$ & $<5$ & $<3$ & - & $<5$ & $<5$ \\
\hline $6-48 \cdot 2-2-3,145$ & $<500$ & 300 & $<10$ & $<5$ & $<5$ & $<5$ & $<5$ & - & $<10$ & $<5$ \\
\hline $6-48.2-2-4,18$ & $<500$ & $<300$ & $<10$ & $<5$ & $<5$ & $<5$ & 8 & - & $<5$ & $<5$ \\
\hline $6-48.2-2-5,16$ & $<500$ & 300 & $<10$ & $<5$ & $<5$ & $<5$ & $<5$ & - & $<10$ & $<5$ \\
\hline $6-48.2-2-6,16$ & $<500$ & $<300$ & $<10$ & $<5$ & $<5$ & $<5$ & $<5$ & - & $<5$ & $<5$ \\
\hline $6-48.2-2, \mathrm{CC}$ & $<500$ & $<100$ & $<10$ & $<5$ & $<5$ & $<5$ & $<3$ & $<10$ & 5 & $<5$ \\
\hline $6-48.2-3-1,117$ & $<500$ & $<300$ & $<10$ & $<5$ & $<5$ & $<5$ & $<5$ & - & $<10$ & $<5$ \\
\hline $6-48 \cdot 2-3-2,28$ & $<500$ & $<300$ & $<10$ & $<5$ & $<5$ & $<5$ & $<5$ & - & $<5$ & $<5$ \\
\hline $6-48.2-3-3,17$ & $<500$ & $<300$ & $<10$ & $<5$ & $<5$ & $<5$ & $<5$ & - & $<10$ & $<5$ \\
\hline
\end{tabular}


TABLE 11 - Continued

\begin{tabular}{|c|c|c|c|c|c|c|c|c|c|c|}
\hline Sample Designation & $\mathrm{Mn}$ & $\mathrm{Ti}$ & $\mathrm{Cr}$ & V & $\mathrm{Ni}$ & Co & $\mathrm{Zr}$ & $\mathrm{Cu}$ & $\mathrm{Pb}$ & Mo \\
\hline $6-48-2-3-4,17$ & $<500$ & $<300$ & $<10$ & $<5$ & $<5$ & $<5$ & $<3$ & - & $<5$ & $<5$ \\
\hline $6-48-2-3-5,16$ & $<500$ & $<300$ & $<10$ & $<5$ & $<5$ & $<5$ & $<5$ & - & $<10$ & $<5$ \\
\hline $6-49.0-1-1,11$ & $>4,000$ & 3,600 & 64 & 96 & 245 & 110 & 105 & - & - & 15.5 \\
\hline $6-49.0-1-1,76$ & 4,900 & 2,600 & 47 & 81 & 81 & 22 & 96 & - & - & 6 \\
\hline $6-49.0-1-2,16$ & $<500$ & 2,900 & 58 & 60 & 70 & 40 & 78 & - & 48 & $<10$ \\
\hline $6-49.0-1-3,16$ & 10,800 & 4,600 & 64 & 150 & 340 & 113 & 270 & - & 22 & 7 \\
\hline $6-49.0-1-4,6$ & 3,800 & 3,300 & 84 & 88 & 14.5 & 54 & 100 & - & - & $<10$ \\
\hline $6-49.0-1-5,7$ & 19,000 & 3,750 & 56 & 136 & 340 & 133 & 270 & - & 110 & 26 \\
\hline $6-49.0-1-5,70$ & 8,000 & 2,000 & 44 & 66 & 195 & 70 & 92 & - & 40 & 20 \\
\hline $6-49.0-1-5,130$ & $<500$ & $<300$ & $<10$ & $<5$ & 5 & $<5$ & 9 & 23 & 48 & $<5$ \\
\hline $6-49.0-1-6,7$ & 26,000 & 4,100 & 54 & 125 & 460 & 230 & 220 & - & 88 & 28 \\
\hline $6-49.0-2-1,70$ & $<500$ & 310 & $<10$ & $<10$ & $<10$ & $<10$ & $<5$ & - & $<10$ & $<10$ \\
\hline $6-49.1-1-1,77$ & 5,400 & 1,950 & 31 & 54 & 96 & 39 & 77 & - & 41 & $<10$ \\
\hline $6-49.1-1-2,16$ & 16,000 & 5,000 & 68 & 163 & 380 & 164 & 330 & - & 76 & 39 \\
\hline $6-49.1-1-2,124$ & 25,000 & 3,200 & 34 & 85 & 640 & 105 & 200 & - & 83 & 53 \\
\hline $6-49.1-1-3,22$ & 23,000 & 3,850 & 50 & 105 & $>500$ & 164 & 180 & - & 68 & 65 \\
\hline $6-49.1-1-4,17$ & 12,500 & 3,400 & 34 & 78 & 430 & 80 & 160 & - & 56 & 44 \\
\hline $6-49.1-1-4,117$ & 5,900 & 1,750 & 16.5 & 46 & 158 & 35 & 54 & - & 14 & 10 \\
\hline $6-49.1-1-5,38$ & 520 & 300 & $<10$ & $<10$ & $<10$ & $<10$ & 7 & - & $<10$ & $<10$ \\
\hline $6-49.1-1-5,60$ & 1,600 & 3,800 & 14 & 88 & 7 & 14 & 54 & 63 & 24 & 8.2 \\
\hline $6-49.1-1-5,60$ & 16,000 & 4,000 & 56 & 120 & 560 & 150 & 225 & 11.5 & 98 & 41 \\
\hline $6-49.1-2-1,145$ & 1,470 & 300 & $<10$ & 13.3 & $<5$ & $<5$ & 6 & - & $<5$ & $<5$ \\
\hline $6-49.1-2-2,145$ & 500 & 420 & $<10$ & 12 & $<10$ & $<10$ & 6 & - & $<10$ & $<10$ \\
\hline $6-49.1-2-3,145$ & 500 & 700 & 14 & 32 & 8.5 & $<5$ & 24 & - & 26 & $<5$ \\
\hline $6-49.1-2, \mathrm{CC}$ & $<500$ & $<300$ & $<10$ & $<5$ & $<5$ & $<5$ & 8 & - & $<5$ & $<5$ \\
\hline $6-50.0-2-1,145$ & 2,200 & 520 & 10 & 19 & 16 & 18 & 9.5 & 210 & 15 & 19 \\
\hline $6-50.0-2-2,150$ & 770 & 390 & 18 & 21 & 14 & 28 & 6 & 270 & 12 & $<5$ \\
\hline $6-50.0-2-3,150$ & 2,100 & 2,100 & $<10$ & 76 & 5 & 7.6 & 48 & 135 & 6 & $<5$ \\
\hline $6-50.0-2-4,150$ & 1,450 & 670 & $<10$ & 23 & 16 & 34 & 14 & 200 & 5 & $<5$ \\
\hline $6-50.0-2-6,150$ & 1,000 & 310 & $<10$ & 25 & 12 & 13 & 13 & 74 & 14 & $<5$ \\
\hline $6-50.0-2, \mathrm{CC}$ & $<500$ & $<300$ & $<10$ & $<5$ & $<5$ & $<5$ & $<5$ & - & $<5$ & $<5$ \\
\hline $6-50.1-1-1,62$ & 4,800 & 3,300 & 60 & 105 & 98 & 31 & 145 & - & 50 & $<10$ \\
\hline $6-50.1-1-2,15$ & 3,000 & 2,600 & 37 & 78 & 70 & 37 & 105 & - & 54 & $<10$ \\
\hline $6-50.1-1-3,7$ & 3,900 & 3,400 & 42 & 112 & 81 & 32 & 146 & - & 28 & $<5$ \\
\hline $6-50.1-1-4,17$ & 2,700 & 2,700 & 63 & 76 & 70 & 35 & 77 & - & 45 & $<10$ \\
\hline $6-50.1-1-5,15$ & 1,800 & 3,170 & 56 & 100 & 75 & 22 & 118 & - & 18 & $<5$ \\
\hline $6-50.1-1-6,16$ & $<500$ & 3,700 & 50 & $110 ?$ & 81 & 27 & 430 & - & 25 & $<5$ \\
\hline $6-50.1-2-1,130$ & 3,850 & 2,900 & 39 & 81 & 85 & 23 & 121 & - & 34 & 8 \\
\hline $6-50.1-2-3,4$ & 5,600 & 2,500 & 40 & 74 & 105 & 43 & 100 & - & 58 & $<10$ \\
\hline $6-50.1-2-3,35$ & 3,300 & 3,300 & 45 & 82 & 105 & 28 & 116 & - & $41 ?$ & $<5$ \\
\hline
\end{tabular}


TABLE 11 - Continued

\begin{tabular}{|c|c|c|c|c|c|c|c|c|c|c|}
\hline Sample Designation & $\mathrm{Mn}$ & $\mathrm{Ti}$ & $\mathrm{Cr}$ & V & $\mathrm{Ni}$ & Co & $\mathrm{Zr}$ & $\mathrm{Cu}$ & $\mathrm{Pb}$ & Mo \\
\hline $6-50.1-2-4,12$ & 8,600 & 3,400 & 60 & 100 & 300 & 71 & 160 & - & 76 & 12 \\
\hline $6-50.1-2-4,50$ & 8,300 & 4,800 & 70 & 105 & 440 & 86 & 190 & 330 & 77 & 32 \\
\hline $6-50.1-2-5,8$ & 6,900 & 4,800 & 55 & 140 & 240 & 69 & 198 & - & 32 & 31 \\
\hline $6-50.1-3-1,145$ & 7,400 & 2,500 & 43 & 113 & 180 & 77 & 190 & 7,500 & 57 & 16 \\
\hline $6-50.1-3-2,76$ & 8,000 & 3,700 & 56 & 105 & 195 & 59 & 120 & 370 & 28 & 32 \\
\hline $6-50.1-3-3,15$ & 9,000 & 3,700 & 53 & 110 & 260 & 67 & 146 & - & 71 & 30 \\
\hline $6-50.1-3-4,16$ & 9,000 & 3,100 & 62 & 80 & 200 & 96 & 295 & - & 31 & 19 \\
\hline $6-50.1-3-5,28$ & 12,800 & 3,700 & 50 & 137 & 330 & 188 & 200 & - & 88 & 24 \\
\hline $6-50.1-3-6,16$ & 9,700 & 3,300 & 43 & 86 & 270 & 109 & 310 & - & 65 & 18 \\
\hline $6-50.1-4-1,75$ & 14,000 & 2,900 & 46 & 84 & 400 & 127 & 310 & - & 64 & 17 \\
\hline $6-51.0-1-1,11$ & 11,100 & 3,670 & 44 & 96 & 135 & 84 & 90 & 380 & 58 & 31 \\
\hline $6-51.0-1-2,8$ & $<23,000$ & 3,800 & 46 & 120 & 170 & 124 & 162 & $>500$ & 23 & 30 \\
\hline $6-51.0-1-3,6$ & 16,000 & 4,300 & 55 & 128 & 270 & 157 & 185 & - & 67 & 33 \\
\hline $6-51.0-1-4,6$ & 15,000 & 3,000 & 46 & 120 & 185 & 113 & 155 & $>500$ & 56 & 32 \\
\hline $6-51.0-1-5,15$ & 20,500 & 4,050 & 40 & 110 & 400 & 360 & 118 & 840 & 90 & 43 \\
\hline $6-51.0-1-6,16$ & $>23,000$ & 3,800 & $<10$ & 123 & 610 & $>500$ & 290 & $>500$ & 80 & 81 \\
\hline $6-51.0-1, \mathrm{CC}$ & 20,000 & 3,800 & 58 & 120 & $>500$ & 190 & 180 & 420 & 150 & 50 \\
\hline $6-51.0-2, \mathrm{CC}$ & 5,200 & 3,700 & 48 & 86 & 200 & 76 & 170 & 79 & 21 & 45 \\
\hline $6-51.1-1-1,15$ & 11,500 & 3,800 & 57 & 131 & 84 & 37 & 160 & - & 35 & $<5$ \\
\hline $6-51.1-1-2,16$ & 1,100 & 2,400 & 37 & 72 & 50 & 14 & 130 & - & $?$ & $<5$ \\
\hline $6-51.1-1-3,30$ & 1,600 & 3,500 & 54 & 128 & 80 & 29 & 110 & - & 27 & $<5$ \\
\hline $6-51.1-1-4,16$ & 8,600 & 2,700 & 62 & 107 & 107 & 46 & 180 & - & 48 & $<5$ \\
\hline $6-51.1-1-4,30$ & 1,700 & 5,100 & 54 & 195 & 77 & 28 & 240 & 120 & 40 & $<5$ \\
\hline $6-51.1-1-5,16$ & 1,320 & 2,900 & 38 & 56 & 43 & 21 & 91 & - & 41 & $<5$ \\
\hline $6-51.1-1-6,16$ & 1,050 & 2,700 & 50 & 78 & 60 & 20 & 340 & - & 33 & $<5$ \\
\hline $6-51.1-2-1,127$ & 18,700 & 3,950 & 37 & 100 & 330 & 250 & 120 & 750 & 140 & 42 \\
\hline $6-51.1-2-2,8$ & 16,800 & 3,100 & 50 & 80 & 370 & 125 & 215 & - & 90 & 26 \\
\hline $6-51 \cdot 1-1 \cdot 1,145$ & 17,500 & 3,750 & 40 & 98 & 340 & 172 & 96 & 630 & 260 & 42 \\
\hline $6-52.0-1-1,27$ & 6,400 & 2,850 & 54 & 115 & 88 & 32 & 130 & - & 49 & 5 \\
\hline $6-52.0-1-2,30$ & 4,600 & 6,200 & 45 & 115 & 57 & 28 & 155 & 165 & 63 & 13.5 \\
\hline $6-52.0-1-2,8$ & 3,650 & 3,000 & 40 & 86 & 58 & 22 & 225 & - & 175 & $<5$ \\
\hline $6-52.0-1-2,30$ & 4,300 & 4,500 & 53 & 145 & 76 & 31 & 195 & 92 & 43 & 13 \\
\hline $6-52.0-1 \cdot 3,8$ & 5,200 & 4,300 & 54 & 120 & 80 & 33 & 160 & - & 39 & 6 \\
\hline $6-52.0-1-4,8$ & 4,400 & 3,300 & 55 & 104 & 77 & 32 & 280 & - & 25 & 14 \\
\hline $6-52.0-1-5,10$ & 5,800 & 4,450 & 60 & 140 & 122 & 92 & 130 & 430 & 28 & 15 \\
\hline $6-52.0-1-6,9$ & 3,500 & 3,500 & 60 & 110 & 75 & 35 & 225 & - & 28 & 5 \\
\hline $6-52.0-2-2,7$ & 5,800 & 3,700 & 58 & 118 & 82 & 45 & 130 & - & 41 & $<5$ \\
\hline $6-52.0-2-3,7$ & 3,000 & 4,000 & 62 & 128 & 71 & 30 & 195 & - & 26 & $<5$ \\
\hline $6-52.0-2-4,8$ & 4,700 & 4,800 & 30 & 144 & 90 & 32 & 105 & 240 & 28 & $<10$ \\
\hline
\end{tabular}


TABLE 11 - Continued

\begin{tabular}{|c|c|c|c|c|c|c|c|c|c|c|}
\hline Sample Designation & $\mathrm{Mn}$ & $\mathrm{Ti}$ & $\mathrm{Cr}$ & V & $\mathrm{Ni}$ & Co & $\mathrm{Zr}$ & $\mathrm{Cu}$ & $\mathrm{Pb}$ & Mo \\
\hline $6-52.0-2-5,18$ & 3,400 & 3,400 & 46 & 86 & 62 & 38 & 260 & - & 23 & $<5$ \\
\hline $6-52 \cdot 0-2-6,145$ & 6,800 & 3,400 & 50 & 128 & 80 & 52 & 158 & - & 43 & $<5$ \\
\hline $6-52.0-2-6,145$ & 4,200 & 5,300 & 38 & 180 & 58 & 32 & 450 & 115 & 6 & $<5$ \\
\hline $6-52.0-2, \mathrm{CC}$ & 4,900 & 4,300 & 35 & 140 & 79 & 53 & 210 & 215 & 73 & $<5$ \\
\hline $6-52.0-3-1,16$ & 4,600 & 2,900 & 37 & 96 & 62 & 38 & 180 & - & 28 & $<5$ \\
\hline $6-52.0-3-2,19$ & 7,200 & 3,670 & 40 & 100 & 70 & 62 & 86 & 320 & 10 & $<10$ \\
\hline $6-52.0-3-3,16$ & 1,850 & 2,700 & 23 & 54 & 66 & 13 & 190 & - & 25 & $<5$ \\
\hline $6-52.0-3-4,16$ & 4,900 & 2,500 & 32 & 88 & 70 & 18.7 & 200 & - & 26 & $<5$ \\
\hline $6-52.0-3-5,7$ & 2,500 & 3,500 & 31 & 104 & 42 & 30 & 205 & - & 28 & $<5$ \\
\hline $6-52.0-3-6,6$ & 3,100 & 3,800 & 37 & 100 & 76 & 31 & 210 & - & 28 & $<5$ \\
\hline $6-52.0-4-2,7$ & 3,700 & 3,500 & 27 & 98 & 50 & 34 & 215 & - & 35 & $<5$ \\
\hline $6-52.0-4-3,5$ & 3,900 & 4,100 & 34 & 145 & 62 & 51 & 175 & - & 31 & $<5$ \\
\hline $6-52.0-4-4,6$ & 3,500 & 3,200 & 25 & 80 & 46 & 29 & 190 & - & 28 & $<5$ \\
\hline $6-52.0-4-5,6$ & 1,370 & 3,900 & 46 & 148 & 60 & 11 & 168 & - & 39 & $<5$ \\
\hline $6-52.0-4-6,18$ & 4,550 & 3,300 & 24 & 95 & 48 & 30 & 185 & - & 33 & $<5$ \\
\hline $6-52.0-4-6,128$ & 1,500 & 2,850 & 29 & 104 & 54 & 6 & 145 & - & 31 & $<5$ \\
\hline $6-52.0-5-1,127$ & 9,000 & 4,650 & 52 & 120 & 125 & 80 & 310 & - & 98 & 20 \\
\hline $6-52.0-5-2,10$ & 6,300 & 4,000 & 36 & 149 & 90 & 53 & 142 & - & 36 & 8 \\
\hline $6-52.0-5-2,17$ & 4,000 & 3,300 & 70 & 98 & 47 & 50 & 69 & - & - & $<10$ \\
\hline $6-52.0-5-3,15$ & 8,200 & 3,000 & 40 & 108 & 106 & 64 & 132 & - & 31 & 18 \\
\hline $6-52.0-5-3,18$ & 4,000 & 2,900 & 88 & 85 & 58 & 57 & 62 & - & - & 10.5 \\
\hline $6-52.0-5-4,18$ & 11,500 & 3,900 & 40 & 152 & 160 & 107 & 204 & - & 43 & 43 \\
\hline $6-52.0-5-5,18$ & 4,000 & 3,400 & 110 & 85 & 78 & 80 & 78 & - & - & 15.5 \\
\hline $6-52.0-6-1,24$ & 9,800 & 3,900 & 40 & 108 & 150 & 100 & 118 & 600 & 50 & 31 \\
\hline $6-52.0-6, \mathrm{CC}$ & 5,600 & 4,500 & 43 & 190 & 270 & 145 & 270 & $>500$ & 100 & 40 \\
\hline $6-52.0-7-2,37$ & 74,000 & 3,100 & 105 & 96 & 280 & 115 & 92 & - & - & 30 \\
\hline $6-52.0-7, \mathrm{CC}$ & 2,600 & 1,150 & $<10$ & 25 & 62 & 16 & 52 & 120 & 5 & 12 \\
\hline $6-52.0-8-1,145$ & 12,600 & 2,500 & 34 & 80 & 370 & 81 & 142 & - & 52 & 27 \\
\hline $6-52.0-8-1,145$ & 9,600 & 2,300 & 20 & 61 & 310 & 66 & 130 & 250 & 56 & 28 \\
\hline $6-52.0-8-2,145$ & $>4,000$ & 2,500 & 120 & 70 & 210 & 80 & 90 & - & - & 13.5 \\
\hline $6-52.0-8-2,145$ & 20,000 & 2,900 & 23 & 110 & 560 & 120 & 170 & 540 & 30 & 37 \\
\hline $6-52.0-8-3,21$ & 12,000 & 2,900 & 25 & 66 & 290 & 86 & 96 & 800 & 10 & 24 \\
\hline $6-52.0-8-3,40$ & 9,200 & 2,400 & 20 & 74 & 340 & 70 & 155 & 230 & 24 & 35 \\
\hline $6-52.0-8-4,145$ & 8,400 & 1,650 & 17 & 43 & 177 & 49 & 70 & - & 27 & 20 \\
\hline $6-52.0-8-5,145$ & 8,800 & 1,700 & 13 & 73 & 300 & 52 & 105 & 860 & 16 & 15.5 \\
\hline $6-52.0-8-5,145$ & 4,300 & 1,400 & 110 & 40 & 118 & 52 & 54 & - & - & $<10$ \\
\hline $6-52.0-8-6,145$ & 11,000 & 2,900 & 37 & 106 & 400 & 117 & 155 & - & 49 & 24 \\
\hline $6-52.0-9, \mathrm{CC}$ & 9,800 & 2,300 & 29 & 78 & 150 & 61 & 120 & 330 & 37 & 14.5 \\
\hline $6-52.0-10, \mathrm{CC}$ & 18,000 & 4,300 & 60 & 150 & 480 & 140 & 210 & 290 & 29 & 43 \\
\hline $6-53-0-1-1,10$ & 1,300 & 3,200 & $<40$ & 125 & 10.5 & 20 & 51 & - & - & $<10$ \\
\hline
\end{tabular}


TABLE 11 - Continued

\begin{tabular}{|c|c|c|c|c|c|c|c|c|c|c|}
\hline Sample Designation & $\mathrm{Mn}$ & $\mathrm{Ti}$ & $\mathrm{Cr}$ & V & $\mathrm{Ni}$ & Co & $\mathrm{Zr}$ & $\mathrm{Cu}$ & $\mathrm{Pb}$ & Mo \\
\hline $6-53-0-1-3,17$ & 1,200 & 2,400 & $<40$ & 63 & $<10$ & 12 & 51 & - & - & $<10$ \\
\hline $6-53.0-2, \mathrm{CC}$ & 1,550 & 2,600 & $<10$ & 58 & $<10$ & 15 & 74 & 64 & 19 & $<10$ \\
\hline $6-53.0-2, \mathrm{CC}$ & 2,000 & 4,250 & $<10$ & 168 & 15 & 27 & 61 & 128 & 11 & $<10$ \\
\hline $6-53.0-2, \mathrm{CC}$ & 1,500 & 6,500 & $<10$ & 280 & 12 & 26 & 100 & 100 & 10 & 11 \\
\hline $6-53.0-3-1,17$ & 1,180 & 3,700 & $<10$ & 147 & 15 & 20 & 55 & 96 & 6.6 & $<10$ \\
\hline $6-53.0-4-2$ & 1,450 & $>6,000$ & $<10$ & 145 & 38 & 18 & 66 & $>1,000$ & $<5$ & $<5$ \\
\hline $6-53.0-6-2,65$ & 1,190 & 510 & 11 & 12 & 38 & $<10$ & 9 & 15.5 & $<10$ & $<5$ \\
\hline $6-53.0-6, \mathrm{CC}$ & 6,400 & 1,250 & $<10$ & $<5$ & 560 & 32 & 32 & 100 & 6.6 & $<5$ \\
\hline $6-53.0-6-2,66$ & 1,800 & 700 & $<10$ & 14 & 38 & $<10$ & 9 & 22 & $<10$ & $<10$ \\
\hline $6-53.0-7-1,150$ & 1,350 & 6,300 & 145 & 250 & 180 & 35 & 70 & 58 & 11 & $<10$ \\
\hline $6-53.0-8, \mathrm{CC}$ & 1,000 & 5,100 & 20 & 230 & 170 & 49 & 72 & 210 & 34 & $<5$ \\
\hline $6-53.1-1-2,7$ & 12,000 & 4,950 & 30 & 173 & 96 & 54 & 105 & 700 & 68 & 20 \\
\hline $6-53.1-1-3,18$ & 8,000 & 4,250 & 38 & 147 & 76 & 40 & 86 & 630 & 58 & 12 \\
\hline $6-53.1-1-5,18$ & 8,000 & 3,600 & 28 & 130 & 76 & 40 & 65 & 360 & 23 & 11 \\
\hline $6-53.1-2-1,145$ & 6,400 & 3,700 & $<10$ & 130 & 9 & 17 & 60 & 500 & 12 & $<10$ \\
\hline $6-53.1-2-2,145$ & 2,400 & 2,200 & $<10$ & 100 & 7 & 17 & 110 & 150 & 23 & $<5$ \\
\hline $6-53.1-2-3,16$ & $>23,000$ & 3,500 & $<10$ & 140 & 35 & 25 & 107 & 96 & $<10$ & 27 \\
\hline $6-53 \cdot 1-2-3,90$ & 5,400 & 5,000 & $<10$ & 180 & 12.5 & 21 & 73 & 560 & 12 & $<10$ \\
\hline $6-53.1-2-5,7$ & 3,500 & 3,750 & $<10$ & 172 & 23 & 29 & 53 & 170 & 19 & $<10$ \\
\hline $6-53.1-3-1,150$ & 22,000 & 76,000 & 32 & 320 & 44 & 35 & 100 & 450 & 12 & 18 \\
\hline $6-53 \cdot 1-3-2,16$ & 17,000 & 4,150 & $<10$ & 186 & 27 & 31 & 68 & 560 & 13 & 26 \\
\hline $6-53.1-3-2,40$ & 12,000 & 5,400 & 76 & 200 & 31 & 26 & 91 & 310 & 20 & 11 \\
\hline $6-53.1-3-4,26$ & 4,200 & 4,100 & $<10$ & 210 & 8 & 24 & 56 & 370 & 12 & $<10$ \\
\hline $6-53.2-1-2,16$ & 2,500 & 8,200 & $<10$ & 127 & 220 & 28 & 70 & 255 & $<10$ & $<5$ \\
\hline $6-53.2-1-4,7$ & 5,900 & 5,200 & $<10$ & 350 & 23 & 34 & 84 & 740 & 8 & $<10$ \\
\hline $6-53.2-1-6,7$ & 5,800 & 4,400 & $<10$ & 220 & 23 & 30 & 86 & 740 & 26 & $<10$ \\
\hline $6-53.2-2, \mathrm{CC}$ & 1,600 & 8,400 & 260 & 93 & 135 & 26 & 130 & 76 & 5 & $<5$ \\
\hline $6-53.2-4, \mathrm{CC}$ & 3,400 & 6,400 & 115 & 100 & 110 & 38 & 100 & 250 & 12 & $<10$ \\
\hline $6-53.2-5, \mathrm{CC}$ & 3,700 & 5,700 & 56 & 62 & 163 & 39 & 130 & 270 & 28 & $<10$ \\
\hline $6-54.0-1-1,5$ & 660 & 410 & $<10$ & 17 & 12 & 9.4 & 8 & 45 & $<5$ & $<5$ \\
\hline $6-54.0-1-1,74$ & 1,900 & 3,300 & $<10$ & 82 & $<10$ & 18 & 66 & 65 & 12 & $<10$ \\
\hline $6-54.0-1-2,7$ & 3,400 & 3,800 & $<10$ & 130 & 7 & 12 & 86 & 125 & 8.6 & $<10$ \\
\hline $6-54.0-1-2,20$ & 2,100 & 4,500 & $<10$ & 140 & 8 & 18 & 77 & 85 & 14 & 9.6 \\
\hline $6-54.0-1-2,20$ & 1,850 & 2,800 & $<10$ & 80 & 34 & 16 & 64 & 90 & 11 & $<10$ \\
\hline $6-54.0-1-3,35$ & 1,400 & 3,800 & $<10$ & 66 & $<10$ & 16 & 68 & 56 & 11 & $<10$ \\
\hline $6-54.0-2-1,17$ & 1,530 & 2,700 & $<10$ & 90 & $<5$ & $<10$ & 80 & 300 & $<10$ & $<5$ \\
\hline $6-54.0-2-2,23$ & 1,800 & 2,500 & $<10$ & 82 & 17 & 19 & 75 & 240 & $<10$ & $<10$ \\
\hline $6-54.0-2-4,16$ & 1,460 & 2,520 & $<10$ & 80 & 5 & 11 & 86 & 92 & $<10$ & $<5$ \\
\hline $6-54.0-3-1,140$ & 1,300 & 1,700 & $<10$ & 40 & 5 & $<5$ & 100 & 84 & $<5$ & $<5$ \\
\hline
\end{tabular}


TABLE 11 - Continued

\begin{tabular}{|c|c|c|c|c|c|c|c|c|c|c|}
\hline Sample Designation & $\mathrm{Mn}$ & $\mathrm{Ti}$ & $\mathrm{Cr}$ & V & $\mathrm{Ni}$ & Co & $\mathrm{Zr}$ & $\mathrm{Cu}$ & $\mathrm{Pb}$ & Mo \\
\hline $6-54.0-4-1,108$ & 1750 & 2,800 & $<10$ & 53 & 14 & 9 & 81 & 52 & 19 & $<10$ \\
\hline $6-54.0-4-2,14$ & 2,300 & 2,900 & $<10$ & 58 & 11 & 16 & 86 & 39 & 11 & $<10$ \\
\hline $6-54.0-6-1,145$ & 1,150 & 2,100 & $<10$ & 42 & 1.5 & 7.6 & 115 & 62 & 5 & $<5$ \\
\hline $6-54.0-6-2,16$ & 1,800 & 2,700 & $<10$ & 50 & $<10$ & 14 & 100 & 50 & $<10$ & $<10$ \\
\hline $6-54.0-6-3,23$ & 3,200 & 3,000 & $<10$ & 73 & 8 & 11 & 92 & 125 & 12 & $<10$ \\
\hline $6-54.0-6-4,16$ & 1,600 & 3,300 & $<10$ & 72 & 18 & 15 & 87 & 52 & $<10$ & $<10$ \\
\hline $6-54.0-6-4,78$ & 1,300 & 2,170 & $<10$ & 43 & $<5$ & $<10$ & 78 & 27 & $<10$ & $<5$ \\
\hline $6-54.0-6-5,17$ & 1,650 & 3,000 & $<10$ & 58 & 12 & 12 & 93 & 33 & $<10$ & $<10$ \\
\hline $6-54.0-7-1,138$ & 4,600 & 3,500 & $<10$ & 100 & 13 & 20 & 140 & 152 & 6 & 13 \\
\hline $6-54.0-7-2,34$ & 2,500 & 2,800 & $<10$ & 42 & 35 & 11 & 96 & 27 & 38 & $<10$ \\
\hline $6-54.0-7-3,16$ & 2,700 & 3,400 & $<10$ & 46 & $<5$ & $<10$ & 13 & 52 & $<10$ & $<5$ \\
\hline $6-54.0-9-1$ & 450 & 2,300 & 45 & 78 & 125 & 20 & 40 & 25 & $<10$ & $<5$ \\
\hline $6-55.0-1-2,7$ & 660 & 240 & $<10$ & 6 & 7 & $<10$ & $<5$ & 14 & $<10$ & $<5$ \\
\hline $6-55.0-1-2,30$ & 870 & 280 & $<10$ & 13 & $<5$ & $<5$ & 5.4 & 18.5 & 5 & $<5$ \\
\hline $6-55.0-1-4,7$ & 860 & 350 & $<10$ & 11 & 16 & $<10$ & 7 & 37 & $<10$ & $<5$ \\
\hline $6-55.0-1-6,7$ & 460 & 420 & $<10$ & 11 & 16 & $<10$ & 12 & 20 & $<10$ & $<5$ \\
\hline $6-55.0-2-2,7$ & $<500$ & $<300$ & $<10$ & 6 & 11 & $<10$ & $<5$ & 17.5 & $<10$ & $<5$ \\
\hline $6-55.0-2-4,15$ & $<500$ & 240 & $<10$ & 6 & 8 & $<10$ & $<5$ & $<10$ & $<10$ & $<5$ \\
\hline $6-55.0-2-5,70$ & $<500$ & $<300$ & $<10$ & 6 & 5 & $<10$ & $<5$ & 10 & $<10$ & $<5$ \\
\hline $6-55.0-3-1,16$ & $<500$ & $<300$ & $<10$ & 6 & $<5$ & $<10$ & $<5$ & 19 & $<10$ & $<5$ \\
\hline $6-55.0-3-4,145$ & $<500$ & $<300$ & $<10$ & 5 & 5 & $<10$ & $<5$ & 10 & $<10$ & $<5$ \\
\hline $6-55.0-3-4,145$ & 430 & 340 & $<10$ & 5 & 6 & $<5$ & 5.2 & 23 & $<5$ & $<5$ \\
\hline $6-55.0-4-1,13$ & $<500$ & $<300$ & $<10$ & $<5$ & 5 & $<10$ & $<5$ & 16.5 & $<10$ & $<5$ \\
\hline $6-55.0-4-3,7$ & $<500$ & $<300$ & $<10$ & 5 & $<5$ & $<10$ & $<5$ & $<10$ & $<10$ & $<5$ \\
\hline $6-55.0-4-5,7$ & $<500$ & $<300$ & $<10$ & $<5$ & $<5$ & $<5$ & $<5$ & $<10$ & $<5$ & $<10$ \\
\hline $6-55.0-5-1,7$ & $<500$ & 310 & $<10$ & $<10$ & $<10$ & 40 & $<5$ & 8 & $<10$ & $<10$ \\
\hline $6-55.0-5-5,7$ & $<500$ & 420 & $<10$ & $<10$ & $<10$ & $<10$ & $<5$ & 24 & 16 & $<10$ \\
\hline $6-55.0-6-3,17$ & 680 & 520 & $<10$ & 7 & 9 & $<5$ & 7 & 19 & $<5$ & $<10$ \\
\hline $6-55.0-7-1,14$ & $<500$ & 700 & 11 & 10 & 11 & $<10$ & 9 & 27 & $<10$ & $<10$ \\
\hline $6-55.0-7-5,19$ & $<500$ & 480 & 12 & 10 & $<10$ & $<10$ & $<5$ & 23 & $<10$ & $<10$ \\
\hline $6-55.0-8-3,16$ & $<500$ & 390 & $<10$ & $<5$ & $<5$ & $<5$ & $<5$ & 16.5 & $<5$ & $<10$ \\
\hline 6-55.0-9, Тор & 460 & 760 & $<10$ & 11 & 8 & $<5$ & 28 & 20 & 5 & $<5$ \\
\hline $6-55.0-10-2,10$ & $<500$ & 660 & 18 & 11 & 10 & $<10$ & 9 & $<10$ & $<10$ & $<10$ \\
\hline $6-55.0-10-5,16$ & $<500$ & 730 & 14 & 10 & $<10$ & $<10$ & 12 & 19.5 & $<10$ & $<10$ \\
\hline $6-55.0-11-3,17$ & $<500$ & 270 & $<10$ & $<5$ & $<5$ & $<5$ & $<5$ & 14.5 & $<5$ & $<10$ \\
\hline $6-55.0-12-1,13$ & 700 & 750 & $<10$ & 9 & 9 & $<5$ & 5 & 39 & $<5$ & $<10$ \\
\hline $6-55.0-12-3,8$ & $<500$ & 310 & $<10$ & $<10$ & $<10$ & $<10$ & $<5$ & 22 & $<10$ & $<10$ \\
\hline $6-55.0-13-1,7$ & $<500$ & 450 & $<10$ & $<10$ & $<10$ & $<10$ & 52 & $<10$ & $<10$ & $<10$ \\
\hline $6-55.0-13-3,40$ & 340 & 3,900 & $<10$ & 6 & $<5$ & $<5$ & 4.2 & 18 & $<5$ & $<5$ \\
\hline $6-55.0-13-5,7$ & $<500$ & $<300$ & $<10$ & $<10$ & $<10$ & $<10$ & $<5$ & 11 & $<10$ & $<10$ \\
\hline
\end{tabular}


TABLE 11 - Continued

\begin{tabular}{|c|c|c|c|c|c|c|c|c|c|c|}
\hline Sample Designation & $\mathrm{Mn}$ & $\mathrm{Ti}$ & $\mathrm{Cr}$ & V & $\mathrm{Ni}$ & Co & $\mathrm{Zr}$ & $\mathrm{Cu}$ & $\mathrm{Pb}$ & Mo \\
\hline $6-55.0-14-2,145$ & $<500$ & $<300$ & $<10$ & $<10$ & $<10$ & $<10$ & $<5$ & 22 & $<10$ & $<10$ \\
\hline $6-55.0-14-5,145$ & $<500$ & 270 & $<10$ & $<5$ & $<5$ & $<5$ & 6 & 37 & $<5$ & $<10$ \\
\hline $6-55.0-14-6,145$ & $<500$ & 310 & $<10$ & $<10$ & $<10$ & $<10$ & $<5$ & 18.5 & $<10$ & $<10$ \\
\hline $6-56.2-1-2,145$ & $<500$ & $<300$ & $<10$ & $<5$ & $<5$ & $<5$ & $<5$ & 44 & $<5$ & $<10$ \\
\hline $6-56.2-1-6,7$ & $<500$ & $<300$ & $<10$ & $<10$ & $<10$ & $<10$ & $<5$ & 65 & $<10$ & $<10$ \\
\hline $6-56.2-2-4,36$ & $<500$ & $<300$ & $<10$ & $<5$ & $<5$ & $<5$ & $<5$ & 10 & $<5$ & $<10$ \\
\hline $6-56.2-3-2,145$ & $<500$ & $<300$ & $<10$ & $<5$ & $<5$ & $<5$ & $<5$ & 26 & $<5$ & $<10$ \\
\hline $6-56.2-3 \cdot 6,145$ & $<500$ & 380 & $<10$ & $<5$ & $<5$ & $<5$ & $<5$ & 52 & $<5$ & $<10$ \\
\hline $6-56.2-4-4,14$ & $<500$ & 330 & $<10$ & $<5$ & 8 & $<5$ & $<5$ & 16.5 & $<5$ & $<10$ \\
\hline $6-56.2-5-2,13$ & $<500$ & 550 & $<10$ & $<5$ & 8 & $<5$ & $<5$ & 19 & $<5$ & $<10$ \\
\hline $6-56.2-5-6,7$ & $<500$ & 430 & $<10$ & $<10$ & $<10$ & $<10$ & $<5$ & 10 & $<10$ & $<10$ \\
\hline $6-56-2-6-2,7$ & $<500$ & 1,110 & $<10$ & 9 & 6.5 & $<5$ & 7 & 32 & $<5$ & $<10$ \\
\hline $6-56.2-7-6,7$ & $<500$ & 510 & $<10$ & $<5$ & $<5$ & $<5$ & $<5$ & 25 & $<5$ & $<10$ \\
\hline $6-56.2-8-4,7$ & $<500$ & 660 & $<10$ & $<10$ & $<10$ & $<10$ & 6 & 9 & $<10$ & $<10$ \\
\hline $6-56.2-9-4,19$ & 480 & 2,800 & $<10$ & 15 & $<10$ & $<10$ & 37 & 10 & $<10$ & $<10$ \\
\hline $6-56.2-10-2,10$ & $<500$ & 750 & $<10$ & $<10$ & $<10$ & $<10$ & 36 & 12.5 & $<10$ & $<10$ \\
\hline $6-56.2-10-6,13$ & 500 & 3,700 & $<10$ & 54 & $<10$ & $<10$ & 155 & $<10$ & $<10$ & $<10$ \\
\hline $6-57.1-1-1$ & $<500$ & $<300$ & $<10$ & $<10$ & $<10$ & $<10$ & $<5$ & $<10$ & $<10$ & $<10$ \\
\hline $6-57.1-1-2,13$ & $<500$ & $<300$ & $<10$ & $<10$ & $<10$ & $<10$ & $<5$ & 11 & $<10$ & $<10$ \\
\hline $6-57.1-1-4,7$ & $<500$ & $<300$ & $<10$ & $<10$ & $<10$ & $<10$ & $<5$ & 9 & $<10$ & $<10$ \\
\hline $6-57.1-1-6,11$ & $<500$ & $<300$ & $<10$ & $<10$ & $<10$ & $<10$ & $<5$ & 23 & $<10$ & $<10$ \\
\hline $6-57.1-3-1,22$ & 520 & 1,360 & 10 & 27 & 11 & $<10$ & 13 & 18 & $<10$ & $<10$ \\
\hline $6-57.1-3-1,17$ & 1,200 & 1,050 & $<10$ & 26 & 9 & $<5$ & 16 & 94 & 16 & $<5$ \\
\hline $6-57.1-4-1,86$ & 720 & 2,250 & 23 & 76 & 19 & 10 & 15 & 31 & $<5$ & $<10$ \\
\hline $6-57.1-4-3,14$ & 800 & 1,770 & 10 & 40 & 13 & $<10$ & 14 & 36 & $<10$ & $<10$ \\
\hline $6-57.1-4-4,145$ & 2,800 & 2,000 & 17.5 & 53 & 12 & 7.8 & 24 & $>500$ & 6 & $<5$ \\
\hline $6-58.1-1-1,139$ & 2,800 & 3,700 & 27 & 89 & 68 & 38 & 64 & 130 & 23 & $<10$ \\
\hline $6-58.1-1-2,91$ & 1,140 & 630 & 14 & 12 & 18 & $<10$ & 14 & 27 & $<10$ & $<10$ \\
\hline $6-58.1-2-5,1$ & 1,340 & 10,500 & 450 & 270 & 188 & 43 & 77 & 120 & $<10$ & 13 \\
\hline $6-58.2-1-2,16$ & $<500$ & 5,300 & 27 & 76 & 40 & 20 & 70 & 82 & $<10$ & $<10$ \\
\hline $6-58.2-1-4,31$ & $<500$ & 8,100 & 120 & 100 & 125 & 33 & 64 & 120 & $<10$ & $<10$ \\
\hline $6-58.2-1-5,0$ & 2,000 & 11,500 & 600 & 450 & 220 & 53 & 190 & 145 & $<5$ & $<5$ \\
\hline $6-58.2-1-6,95$ & 1,300 & 3,800 & 16 & 32 & 13 & 6 & 380 & 50 & 7 & $<5$ \\
\hline $6-59.1-3-1,130$ & 8,000 & 7,700 & 74 & 165 & 165 & 79 & 170 & 260 & 39 & $<10$ \\
\hline $6-59.1-3-3,7$ & 6,800 & 6,200 & 39 & 120 & 135 & 63 & 110 & 200 & 28 & 27 \\
\hline $6-59.1-3, \mathrm{CC}$ & 8,500 & 6,400 & 60 & 155 & 195 & 68 & 220 & 400 & 59 & 17.5 \\
\hline $6-59.2-1-1,47$ & 2,000 & 10,800 & 135 & 150 & 125 & 35 & 106 & 138 & $<10$ & $<10$ \\
\hline $6-59.2-1-3,4$ & 2,200 & 9,700 & 83 & 145 & 90 & 41 & 146 & 190 & $<10$ & $<10$ \\
\hline $6-59.2-1, \mathrm{CC}$ & 3,500 & 12,500 & 200 & 160 & 138 & 35 & 300 & 200 & 5 & $<5$ \\
\hline $6-59.2-2-2,145$ & 1,250 & 6,600 & 120 & 95 & 80 & 28 & 67 & 172 & $<10$ & $<10$ \\
\hline
\end{tabular}


TABLE 11 - Continued

\begin{tabular}{|c|c|c|c|c|c|c|c|c|c|c|}
\hline Sample Designation & $\mathrm{Mn}$ & $\mathrm{Ti}$ & $\mathrm{Cr}$ & V & $\mathrm{Ni}$ & Co & $\mathrm{Zr}$ & $\mathrm{Cu}$ & $\mathrm{Pb}$ & Mo \\
\hline $6-59.2-2-4,4$ & 1,730 & 9,500 & 138 & 137 & 135 & 39 & 106 & 200 & 25 & $<10$ \\
\hline $6-59.2-2-6,16$ & 1,200 & 5,800 & 117 & 117 & 112 & 30 & 110 & 120 & $<10$ & $<10$ \\
\hline 6-59.2-3, Тор & 2,100 & 7,100 & 100 & 98 & 58 & 24 & 100 & 170 & 13 & $<5$ \\
\hline $6-59.2-3-1,145$ & 2,300 & 9,000 & 125 & 145 & 100 & 39 & 115 & 180 & 25 & $<10$ \\
\hline $6-59.2-3, \mathrm{CC}$ & 2,600 & 11,500 & 130 & 160 & 240 & 48 & 134 & 230 & 14 & 9 \\
\hline $6-59.2-4-1,66$ & 2,200 & 5,900 & 105 & 105 & 92 & 34 & 73 & 120 & $<10$ & $<10$ \\
\hline $6-59.2-4, \mathrm{CC}$ & 3,400 & 12,000 & 185 & 170 & 240 & 54 & 140 & 200 & 25 & 9 \\
\hline $6-59.2-5-1,89$ & 4,900 & 2,650 & 25 & 62 & 195 & 53 & 60 & 250 & 35 & $<10$ \\
\hline $6-59.2-5, \mathrm{CC}$ & 6,900 & 8,600 & 58 & 93 & 390 & 55 & 190 & 200 & 29 & 13 \\
\hline 6-59.2-6, Тop? & 1,430 & 6,000 & 185 & 108 & 69 & 29 & 78 & - & $<5$ & $<5$ \\
\hline 6-59.2-6, Тор & 23,000 & 5,400 & 50 & 100 & 380 & 87 & 190 & $>500$ & 48 & 15 \\
\hline 6-59.2-6, Тор & 4,600 & 3,100 & 16 & 77 & 199 & 62 & 73 & 165 & 40 & $<10$ \\
\hline 6-59.2-6, Тор & 6,200 & 2,700 & 15 & 54 & 250 & 58 & 140 & 160 & 61 & 13 \\
\hline $6-59.2-6-2,0$ & 4,350 & 5,200 & 52 & 105 & 123 & 38 & 110 & 114 & 33 & $<10$ \\
\hline $6-60.0-1-2,7$ & $<500$ & 1,550 & 20 & 92 & 25 & $<10$ & 28 & 16 & $<10$ & $<10$ \\
\hline $6-60.0-1-3,10$ & $<500$ & 1,230 & $<10$ & 50 & 18 & $<10$ & 25 & 10 & $<10$ & $<10$ \\
\hline $6-60.0-1, \mathrm{CC}$ & 340 & 3,300 & 98 & 90 & 60 & 17 & 53 & 74 & $<5$ & $<5$ \\
\hline $6-60.0-2-2,12$ & $<500$ & 2,300 & $<10$ & 105 & 11 & $<10$ & 38 & 37 & $<10$ & $<10$ \\
\hline $6-60.0-3-1,117$ & $<500$ & 1,480 & $<10$ & 54 & $<10$ & $<10$ & 54 & 70 & $<10$ & $<10$ \\
\hline $6-60.0-3, \mathrm{CC}$ & 2,900 & 4,900 & $<10$ & 200 & 14 & 24 & 58 & $>500$ & 32 & 14 \\
\hline $6-60.0-4-1$ & 500 & 2,160 & $<10$ & 96 & $<10$ & $<10$ & 47 & 34 & $<10$ & $<10$ \\
\hline $6-60.0-4-1,145$ & 1,650 & 3,300 & $63 ?$ & 125 & 10 & 15 & 81 & 160 & 30 & 7 \\
\hline $6-60.0-4-2,145$ & 1,800 & 4,200 & $<10$ & 83 & $<5$ & 11 & 84 & 74 & 14 & $<5$ \\
\hline $6-60.0-4, \mathrm{CC}$ & 1,500 & 3,500 & $<10$ & 80 & 6 & 9 & 115 & 92 & 25 & $<5$ \\
\hline $6-60.0-5-2,26$ & $<500$ & 1,270 & $<10$ & 30 & $<10$ & $<10$ & 58 & 16.5 & $<10$ & $<10$ \\
\hline $6-60.0-5, \mathrm{CC}$ & 1,650 & 3,900 & $<10$ & 61 & 6 & 11 & 84 & 56 & 10 & $<5$ \\
\hline $6-60.0-6-1,86$ & $<500$ & 2,500 & $<10$ & 112 & $<10$ & 11 & 35 & 34 & 23 & $<10$ \\
\hline $6-60.0-6-3,14$ & 510 & 3,350 & $<10$ & 120 & $<10$ & 13 & 47 & 37 & $<10$ & $<10$ \\
\hline $6-60.0-6-5,37$ & $<500$ & 2,300 & $<10$ & 68 & $<10$ & $<10$ & 65 & 52 & $<10$ & $<10$ \\
\hline $6-60.0-6-7,90$ & $<500$ & 2,200 & $<10$ & 34 & $<10$ & $<10$ & 72 & 28 & $<10$ & $<10$ \\
\hline $6-60 \cdot 0-7-2,7$ & 530 & 2,000 & $<10$ & 23 & $<10$ & 10 & 78 & 16 & $<10$ & $<10$ \\
\hline $6-60.0-8-2,72$ & 660 & 3,500 & $<10$ & 133 & $<10$ & 11 & 60 & 33 & $<10$ & $<10$ \\
\hline $6-60.0-9-2,145$ & 660 & 4,200 & $<10$ & 148 & 11 & 12 & 60 & 37 & $<10$ & $<10$ \\
\hline
\end{tabular}


TABLE 12

Results of the Analysis of $\mathrm{Fe}_{2} \mathrm{O}_{3}, \mathrm{TiO}_{2}$, and $\mathrm{MnO}(\%)$

\begin{tabular}{|c|c|c|c|c|c|c|c|}
\hline Sample Designation & $\mathrm{Fe}_{2} \mathrm{O}_{3}$ & $\mathrm{TiO}_{2}$ & $\mathrm{MnO}$ & Sample Designation & $\mathrm{Fe}_{2} \mathrm{O}_{3}$ & $\mathrm{TiO}_{2}$ & $\mathrm{MnO}$ \\
\hline $44.0-1-1,145$ & 0.26 & \multirow{13}{*}{0.00} & 0.02 & $47.2-2-3,7$ & 1.12 & Tr. & 0.06 \\
\hline $44.0-1-2,145$ & 0.26 & & 0.02 & $47.2-2-5,5$ & 1.28 & Tr. & 0.06 \\
\hline $44.0-1-3,145$ & 0.22 & & 0.02 & $47.2-3-\mathrm{CC}$ & 0.96 & 0.06 & 0.06 \\
\hline 44.0-1-3, Mixed & 0.19 & & 0.02 & $47.2-3-2,6.5$ & 1.79 & Tr. & 0.05 \\
\hline $44.0-1-3,145$ & 0.19 & & 0.02 & $47.2-3-4,16$ & 1.28 & Tr. & 0.07 \\
\hline $44.0-1-4,145$ & 0.38 & & 0.02 & $47.2-4-\mathrm{CC}$ & 1.02 & & 0.07 \\
\hline $44.0-2-\mathrm{CC}$ & 0.25 & & 0.03 & $47.2-4-2,17$ & 0.70 & Tr. & 0.07 \\
\hline $44.0-2-1,145$ & 0.45 & & 0.02 & $47.2-4-4,16.5$ & 1.28 & Tr. & 0.06 \\
\hline $44.0-2-2,145$ & 0.19 & & 0.02 & $47.2-4-6,17$ & 0.80 & 0.08 & 0.09 \\
\hline $44.0-2-3,145$ & 0.32 & & 0.02 & $47.2-5-2,6$ & 0.83 & Tr. & 0.08 \\
\hline $44.0-2-4,145$ & 0.26 & & 0.03 & $47.2-5-4,6$ & 1.75 & 0.13 & 0.10 \\
\hline $44.0-2-5,145$ & 0.26 & & 0.02 & $47.2-5-6,6.5$ & 1.15 & 0.08 & 0.09 \\
\hline $44.0-2-6,145$ & 0.26 & & 0.02 & $47.2-6-3,17$ & 0.96 & 0.10 & 0.10 \\
\hline $44.0-3-\mathrm{CC}$ & 0.77 & 0.00 & 0.09 & $47.2-7-2,17$ & 0.32 & Tr. & 0.06 \\
\hline $44.0-3-\mathrm{CC}$ & 0.76 & 0.00 & 0.10 & $47.2-7-4,17$ & 0.38 & Tr. & 0.06 \\
\hline $44.0-3-2,145$ & 0.26 & \multirow{13}{*}{0.00} & 0.03 & $47.2-7-6,126$ & 0.45 & Tr. & 0.06 \\
\hline $44.0-3-3,145$ & 0.26 & & 0.03 & $47.2-8-1,150$ & 0.31 & 0.00 & 0.05 \\
\hline $44.0-3-4,145$ & 0.26 & & 0.03 & $47.2-8-2,17$ & 0.32 & & 0.05 \\
\hline $44.0-3-5,145$ & 0.26 & & 0.03 & $47.2-8-4,17$ & 0.39 & Tr. & 0.03 \\
\hline $44.0-4-\mathrm{CC}$ & 0.25 & & 0.03 & $47.2-8-6-\mathrm{CC}$ & 0.70 & 0.00 & 0.05 \\
\hline 44.0-4-0, Тор & 0.60 & & 0.03 & $47.2-8-6,16$ & 0.29 & Tr. & 0.03 \\
\hline 44.0-4-0, Тор & 0.66 & & 0.04 & $47.2-9-\mathrm{CC}$ & 0.77 & 0.00 & 0.11 \\
\hline $44.0-4-2,145$ & 0.45 & & 0.03 & $47.2-9-2,17$ & 0.38 & & 0.05 \\
\hline $44.0-4-3,145$ & 0.26 & & 0.02 & $47.2-9-4,0$ & 0.26 & 0.0 & 0.05 \\
\hline $44.0-4-4,145$ & 0.19 & & 0.03 & $47.2-9-5,17$ & 0.38 & Tr. & 0.05 \\
\hline $44.0-4-5,145$ & 0.32 & & 0.05 & $47.2-9-5,150$ & 0.32 & & 0.04 \\
\hline $44.0-4-6,145$ & 0.26 & & 0.03 & $47.2-9-6,0$ & 0.32 & 0.00 & 0.06 \\
\hline $44.0-5-0$ & 4.12 & & 0.09 & $47.2-10$, Top & 0.22 & 0.00 & 0.04 \\
\hline $45.1-1-2,100$ & 11.55 & 1.57 & 1.31 & $47.2-10-\mathrm{CC}$ & 0.57 & 0.00 & 0.07 \\
\hline $45.1-1-2,124$ & 12.44 & 2.36 & 0.18 & $47.2-10-1,17$ & 0.32 & & 0.03 \\
\hline $45.1-1-3,23$ & 12.19 & 2.32 & 0.15 & $47.2-10-3,18$ & 0.26 & & 0.03 \\
\hline $45.1-1-4,22$ & 12.57 & 2.29 & 0.16 & $47.2-10-5,17$ & 0.57 & Tr. & 0.11 \\
\hline $45.1-1-4,136$ & 7.85 & 1.41 & 0.12 & $47.2-11-\mathrm{CC}$ & 0.31 & 0.00 & 0.02 \\
\hline $45.1-1-5,4$ & 10.65 & 2.00 & 0.22 & $47.2-11,145$ & 1.27 & 0.06 & 0.10 \\
\hline $45.1-2-\mathrm{CC}$ & 3.25 & 0.38 & 0.45 & $47.2-11-1,145$ & 0.26 & & 0.02 \\
\hline 45.1-2-CC, Total & 3.13 & 0.34 & 0.31 & $47.2-11-3,145$ & 0.31 & 0.00 & 0.03 \\
\hline 46.0 & 1.34 & Tr. & 0.02 & $47.2-11-4,145$ & 0.26 & 0.00 & 0.03 \\
\hline $46.0-1-2,145$ & 7.50 & 0.77 & 0.62 & $47.2-11-5,145$ & 0.26 & & 0.03 \\
\hline $46.0-1-6$ & 1.08 & 0.13 & Tr. & $47.2-11-6,145$ & 0.32 & 0.00 & 0.02 \\
\hline $46.0-1-5,145$ & 5.68 & 0.48 & 0.31 & $47.2-12-\mathrm{CC}$ & 0.22 & 0.00 & 0.02 \\
\hline $47.0-1-2,5$ & 3.99 & 0.34 & 0.03 & $47.2-12-1,145$ & 0.26 & & 0.02 \\
\hline $47.0-1-4,95$ & 2.55 & 0.27 & 0.05 & $47.2-12-3,145$ & 0.26 & & 0.02 \\
\hline 47.1, Top & 0.96 & 0.00 & 0.09 & $47.2-13-\mathrm{CC}$ & 0.31 & 0.00 & 0.02 \\
\hline $47.2-1-3,11$ & 2.23 & 0.21 & 0.06 & $47.2-13-1,145$ & 0.26 & & 0.02 \\
\hline $47.2-1-4,7$ & 2.04 & 0.16 & 0.05 & $47.2-13-3,16$ & 0.26 & & 0.02 \\
\hline $47.2-2-1,145$ & 0.22 & 0.00 & 0.02 & $47.2-13-4,145$ & 0.26 & Tr. & 0.03 \\
\hline
\end{tabular}


TABLE 12 - Continued

\begin{tabular}{|c|c|c|c|c|c|c|c|}
\hline Sample Designation & $\mathrm{Fe}_{2} \mathrm{O}_{3}$ & $\mathrm{TiO}_{2}$ & $\mathrm{MnO}$ & Sample Designation & $\mathrm{Fe}_{2} \mathrm{O}_{3}$ & $\mathrm{TiO}_{2}$ & $\mathrm{MnO}$ \\
\hline $47.2-13-5,145$ & 0.32 & 0.00 & 0.03 & $51.1-1-1,15$ & 6.54 & 0.59 & 1.01 \\
\hline $47.2-14-\mathrm{CC}$ & 0.25 & 0.00 & 0.02 & $52.0-6-\mathrm{CC}$ & 7.72 & 0.64 & 1.12 \\
\hline $47.2-14-1,145$ & 0.26 & 0.00 & 0.02 & $52.0-7-\mathrm{CC}$ & 1.91 & 0.11 & 0.37 \\
\hline $47.2-14-2,145$ & 0.26 & & Tr. & $53.0-2-\mathrm{CC}$ & 8.48 & 0.76 & 0.14 \\
\hline $47.2-14-3,145$ & 0.26 & 0.00 & 0.02 & $53.0-4-2$ & 10.21 & 0.61 & 0.10 \\
\hline $47.2-14-5,145$ & 0.26 & & 0.02 & $53.0-6-\mathrm{CC}$ & 2.42 & 0.16 & 0.48 \\
\hline $48.1-1,28$ & 0.80 & Tr. & 0.07 & $53.0-8-\mathrm{CC}$ & 8.49 & 1.16 & 0.07 \\
\hline $48.1-1-1,26$ & 1.85 & 0.16 & 0.15 & $53.1-2-2,145$ & 6.19 & 0.50 & 0.18 \\
\hline $48.2-1-\mathrm{CC}$ & 0.31 & 0.00 & 0.03 & $53.1-3-1,150$ & 9.57 & 0.70 & 1.62 \\
\hline $48.2-1-3,16$ & 1.08 & 0.06 & 0.07 & $54.0-1-1,5$ & 1.47 & Tr. & 0.11 \\
\hline $48.2-1-5,14.5$ & 0.99 & 0.06 & 0.07 & $54.0-3-1,140$ & 4.15 & 0.45 & 0.14 \\
\hline $48.2-2-\mathrm{CC}$ & 0.25 & 0.00 & 0.01 & $54.0-6-1,145$ & 5.10 & 0.48 & 0.14 \\
\hline $48.2-2-4,18$ & 0.26 & & 0.02 & $54.0-12,20$ & 6.70 & 0.53 & 0.13 \\
\hline $48.2-3-4,17$ & 0.26 & Tr. & 0.02 & $55.0-1-2,30$ & 0.89 & 0.00 & 0.10 \\
\hline $49.0-1-1,76$ & 4.47 & 0.42 & 0.20 & $55.0-3-4,145$ & 0.45 & Tr. & 0.02 \\
\hline $49.0-1-6,7$ & 7.18 & 0.66 & 1.15 & $55.0-9$, Тор & 0.89 & Tr. & 0.03 \\
\hline $49.1-1-5,60$ & 5.49 & 0.56 & 0.14 & $55.0-13-3,40$ & 0.45 & 0.00 & 0.03 \\
\hline $49.1-2-1,145$ & 1.12 & & 0.16 & $57.1-3-1,148$ & 1.60 & 0.16 & 0.10 \\
\hline $50.0-2-1,145$ & 1.72 & Tr. & 0.11 & $57.1-4-4,145$ & 2.49 & 0.24 & 0.12 \\
\hline $50.0-2-2,150$ & 1.60 & 0.08 & 0.14 & $58.2-1-6,95$ & 8.04 & 0.99 & 0.15 \\
\hline $50.0-2-3,150$ & 7.50 & 0.59 & 0.23 & $59.2-3-\mathrm{CC}$ & 10.85 & 1.57 & 0.35 \\
\hline $50.0-2-4,150$ & 1.02 & Tr. & 0.10 & $59.2-5-\mathrm{CC}$ & 6.83 & 1.00 & 0.43 \\
\hline $50.0-2-6,150$ & 1.44 & Tr. & 0.13 & 59.2-6, Тор & 5.30 & 0.51 & 0.83 \\
\hline $50.1-1-3,7$ & 5.23 & 0.48 & 0.31 & $60.0-3-\mathrm{CC}$ & 9.00 & 0.75 & 0.15 \\
\hline $50.1-1-5,17$ & 4.72 & 0.45 & 0.16 & $60.0-4-\mathrm{CC}$ & 7.46 & 0.64 & 0.16 \\
\hline $50.1-2-1,130$ & 6.38 & 0.48 & 0.37 & $60.0-4-\mathrm{CC}$ & 7.43 & 0.64 & 0.17 \\
\hline $50.1-2-3,35$ & 6.48 & 0.56 & 0.45 & $60.0-4-1,145$ & 6.54 & 0.51 & 0.18 \\
\hline $50.1-3-3,15$ & 6.76 & 0.66 & 0.87 & $60.0-4-2,145$ & 6.57 & 0.64 & 0.15 \\
\hline $51.0-1-\mathrm{CC}$ & 7.15 & 0.58 & 1.70 & $60.0-5-\mathrm{CC}$ & 5.61 & 0.50 & 0.20 \\
\hline
\end{tabular}


TABLE 13

Results of Analysis of Calcium Oxide and Magnesium Oxide

\begin{tabular}{|c|c|c|c|c|c|c|c|}
\hline Sample Designation & $\begin{array}{r}\mathrm{CaO} \\
(\%)\end{array}$ & $\begin{array}{l}\mathrm{MgO} \\
(\%)\end{array}$ & $\mathrm{CaO} / \mathrm{MgO}$ & Sample Designation & $\begin{array}{c}\mathrm{CaO} \\
(\%)\end{array}$ & $\begin{array}{l}\mathrm{MgO} \\
(\%)\end{array}$ & $\mathrm{CaO} / \mathrm{MgC}$ \\
\hline $44.0-0-3,50$ & 54.88 & 0.08 & 686.00 & $47.2-5-4,6$ & 45.76 & 0.19 & 240.84 \\
\hline $44.0-1-1,145$ & 54.96 & NIL & & $47.2-5-6,6.5$ & 48.64 & 0.12 & 405.33 \\
\hline $44.0-1-2,145$ & 55.12 & NIL & & $47.2-6-3,17$ & 49.40 & 0.16 & 308.75 \\
\hline $44.0-1-3,145$ & 54.80 & 0.10 & 548.00 & $47.2-7-2,17$ & 55.04 & 0.13 & 423.38 \\
\hline 44.0-1-3, Mixed & 54.72 & NIL & & $47.2-7-4,17$ & 53.92 & 0.13 & 414.77 \\
\hline $44.0-1-4,145$ & 54.64 & 0.10 & 546.40 & $47.2-7-6,126$ & 53.88 & 0.14 & 384.86 \\
\hline $44.0-2-\mathrm{CC}$ & 55.28 & 0.06 & 921.33 & $47.2-8-2,17$ & 54.88 & 0.25 & 219.52 \\
\hline $44.0-2-1,145$ & 55.28 & NIL & & $47.2-8-4,17$ & 55.20 & 0.24 & 230.00 \\
\hline $44.0-2-2,145$ & 54.44 & NIL & & $47.2-8-6,16$ & 54.56 & 0.07 & 779.43 \\
\hline $44.0-2-3,145$ & 54.96 & 0.10 & 549.60 & $47.2-8-6$ & 54.36 & 0.10 & 543.60 \\
\hline $44.0-2-4,145$ & 55.00 & 0.07 & 785.71 & 47.2-9-CC & 52.20 & 0.10 & 522.00 \\
\hline $44.0-2-5,145$ & 54.88 & NIL & & $47.2-9-1$ & 54.44 & NIL & \\
\hline $44.0-2-6,145$ & 54.88 & NIL & & $47.2-9-2,17$ & 55.28 & 0.10 & 552.80 \\
\hline $44.0-3-\mathrm{CC}$ & 43.12 & NIL & & $47.2-9-4,0$ & 54.68 & NIL & \\
\hline $44.0-3-2,145$ & 55.16 & NIL & & $47.2-9-5,17$ & 54.36 & 0.16 & 339.75 \\
\hline $44.0-3-3,145$ & 55.40 & NIL & & $47.2-9-5,150$ & 53.80 & NIL & \\
\hline $44.0-3-4,145$ & 55.20 & NIL & & $47.2-9-6,0$ & 53.88 & NIL & \\
\hline $44.0-3-5,145$ & 47.68 & NIL & & $47.2-10$, Top & 54.88 & NIL & \\
\hline 44.0-4-0, Тор & 54.88 & 0.09 & 609.78 & $47.2-10-\mathrm{CC}$ & 53.72 & 0.12 & 447.67 \\
\hline $44.0-4-\mathrm{CC}$ & 54.36 & 0.12 & 453.00 & $47.2-10-1,17$ & 54.68 & 0.16 & 341.75 \\
\hline $44.0-4-\mathrm{CC}$ & 54.32 & 0.14 & 388.00 & $47.2-10-3,18$ & 54.24 & 0.12 & 452.00 \\
\hline $44.0-4-2,145$ & 55.00 & NIL & & $47.2-10-5,17$ & 54.04 & 0.13 & 415.69 \\
\hline $44.0-4-3,145$ & 55.08 & NIL & & $47.2-11-\mathrm{CC}$ & 54.96 & 0.06 & 916.00 \\
\hline $44.0-4-4,145$ & 54.60 & NIL & & $47.2-11-1,145$ & 54.64 & 0.25 & 218.56 \\
\hline $44.0-4-5,145$ & 55.28 & 0.09 & 614.22 & $47.2-11-3,145$ & 54.20 & 0.10 & 542.00 \\
\hline $44.0-4-6,145$ & 54.24 & NIL & & $47.2-11-4,145$ & 54.80 & 0.06 & 913.33 \\
\hline $44.0-5-0$ & 46.56 & 0.09 & 517.33 & $47.2-11-5,145$ & 55.68 & 0.16 & 348.00 \\
\hline $45.1-1-3,23$ & 0.64 & 2.63 & 0.24 & $47.2-11-6,145$ & 55.20 & 0.06 & 920.00 \\
\hline $45.1-1-4,22$ & 0.60 & 2.66 & 0.23 & $47.2-12-\mathrm{CC}$ & 55.20 & 0.07 & 788.57 \\
\hline $45.1-1-5,4$ & 6.06 & 3.06 & 1.98 & $47.2-12-1,145$ & 54.56 & 0.16 & 341.00 \\
\hline $47.0-1-5,3.5$ & 40.48 & 0.98 & & $47.2-12-3,145$ & 55.20 & 0.12 & 460.00 \\
\hline 47.1 , Top & 52.76 & 0.09 & 586.22 & $47.2-13-\mathrm{CC}$ & 55.56 & 0.08 & 694.50 \\
\hline $47.1-1-1,145$ & 48.24 & 0.20 & 241.20 & $47.2-13-\mathrm{CC}$ & 55.60 & 0.07 & 794.29 \\
\hline $47.2-1-3,11$ & 40.40 & 0.16 & 252.50 & $47.2-13-1,145$ & 54.44 & 0.16 & 340.25 \\
\hline $47.2-1-4,7$ & 41.96 & 0.30 & 139.87 & $47.2-13-3,16$ & 54.72 & 0.19 & 288.00 \\
\hline $47.2-2-3$ & 47.76 & 0.43 & 111.07 & $47.2-13-4,145$ & 54.44 & 0.16 & 340.25 \\
\hline $47.2-2-5,5$ & 47.68 & 0.17 & 280.47 & $47.2-13-5,145$ & 54.92 & 0.09 & 610.22 \\
\hline $47.2-3-2,6.5$ & 43.48 & 0.43 & 88.73 & $47.2-14-\mathrm{CC}$ & 55.20 & 0.13 & 424.62 \\
\hline $47.2-3-4,16$ & 48.24 & 0.31 & 155.61 & $47.2-14-1,145$ & 55.12 & NIL & \\
\hline $47.2-4-\mathrm{CC}$ & 48.60 & 0.19 & 255.79 & $47.2-14-2,145$ & 54.44 & 0.17 & 320.24 \\
\hline $47.2-4-2,17$ & 52.08 & 0.06 & 868.00 & $47.2-14-3,145$ & 55.08 & 0.07 & 786.86 \\
\hline $47.2-4-4,76.5$ & 48.00 & 0.23 & 208.70 & $47.2-14-5,145$ & 55.08 & 0.13 & 423.69 \\
\hline $47.2-4-6,17$ & 51.24 & NIL & & $48.2-1-\mathrm{CC}$ & 53.20 & 0.13 & 409.23 \\
\hline $47.2-5-2,6$ & 51.04 & 0.07 & 729.14 & $48.2-1-5,14.5$ & 47.88 & 0.22 & 217.64 \\
\hline
\end{tabular}


TABLE 13 - Continued

\begin{tabular}{|c|c|c|c|c|c|c|c|}
\hline Sample Designation & $\begin{array}{l}\mathrm{CaO} \\
(\%)\end{array}$ & $\begin{array}{l}\mathrm{MgO} \\
(\%)\end{array}$ & $\mathrm{CaO} / \mathrm{MgO}$ & Sample Designation & $\begin{array}{l}\mathrm{CaO} \\
(\%)\end{array}$ & $\begin{array}{l}\mathrm{MgO} \\
(\%)\end{array}$ & $\mathrm{CaO} / \mathrm{MgO}$ \\
\hline $48.2-2-\mathrm{CC}$ & 55.48 & 0.12 & 452.33 & $55.0-13-5,7$ & 53.76 & NIL & \multirow{9}{*}{161.87} \\
\hline $48.2-2-1,145$ & 54.56 & 0.12 & 454.67 & $57.1-1-1,10$ & 52.16 & 0.27 & \\
\hline $48.2-2-4,18$ & 54.48 & 0.14 & \multirow[t]{3}{*}{389.14} & $57.1-1-2,13$ & 53.76 & NIL & \\
\hline $48.2-2-6,16$ & 54.72 & 0.24 & & $57.1-1-4,7$ & 52.72 & 0.25 & \\
\hline $48.2-3-2,28$ & 54.48 & NIL & & $57.1-1-6,11$ & 53.52 & 0.82 & \\
\hline $49.1-1-5,60$ & 4.59 & 0.26 & 17.65 & $57.1-3-1,17$ & 48.56 & 0.30 & \\
\hline $50.0-2-1,145$ & 44.96 & 0.28 & 160.57 & $57.1-3-4,22$ & 48.08 & 0.78 & \\
\hline $50.0-2-2,150$ & 44.32 & 0.19 & 233.26 & $57.1-41,86$ & 40.32 & 1.55 & \\
\hline $50.0-2-3,150$ & 1.96 & 0.51 & 3.84 & $57.1-4-3,14$ & 45.12 & 0.83 & \\
\hline $50.0-2-4,150$ & 34.32 & 1.65 & 20.80 & $57.1-4-4,145$ & 44.40 & 0.79 & \multirow[t]{13}{*}{562.03} \\
\hline $50.0-2-6,150$ & 28.80 & 0.13 & 221.54 & $58.1-1-2,91$ & 53.28 & 1.13 & \\
\hline $52.0-1-5,10$ & 1.08 & 2.38 & \multirow{8}{*}{2.00} & $58.2-1-2,16$ & 36.32 & 1.29 & \\
\hline $52.0-2-6,145$ & 0.64 & 2.54 & & $58.2-1-4,31$ & 20.84 & 2.77 & \\
\hline $52.0-3-2,19$ & 0.64 & 2.03 & & $58.2-1-5,0$ & 7.06 & 3.80 & \\
\hline $52.0-7-\mathrm{CC}$ & 0.54 & 0.27 & & $58.2-1-5,1$ & 6.40 & 4.06 & \\
\hline $53.0-1-2,144$ & 1.96 & 1.54 & & $59.2-1-3,4$ & 0.72 & 2.09 & \\
\hline $53.0-2-\mathrm{CC}$ & 2.76 & 1.59 & & $59.2-2-2,145$ & 0.88 & 2.38 & \\
\hline $53.0-3-1,17$ & 2.28 & 1.94 & & $59.2-4-1,66$ & 1.44 & NIL & \\
\hline $53.0-6-2,65$ & 45.68 & 1.01 & & $59.2-5-1,89$ & 3.16 & 2.19 & \\
\hline $53.0-6-\mathrm{CC}$ & 36.64 & 2.64 & \multirow[t]{4}{*}{13.88} & 59.2-6-Тор & 2.68 & 2.86 & \\
\hline $53.1-2-1,145$ & 2.64 & 0.70 & & $60.0-1-2,7$ & 36.00 & 1.01 & \\
\hline $53.1-2-2,145$ & 2.48 & & & $60.0-1-3,10$ & 34.80 & 0.25 & \\
\hline $53.1-2-5,7$ & 1.84 & 0.93 & & $60.0-1-\mathrm{CC}$ & 18.88 & 1.72 & \multirow[t]{3}{*}{10.98} \\
\hline $53.1-3-1,150$ & 1.96 & 0.67 & \multirow[t]{5}{*}{2.93} & $60.0-2-2,12$ & 15.52 & 1.43 & \\
\hline $53.1-3-2,16$ & 1.88 & 1.38 & & $60.0-3-1,117$ & 1.74 & 0.71 & \\
\hline $53.1-3-4,26$ & 1.24 & 0.90 & & $60.0-3-\mathrm{CC}$ & 4.82 & 1.00 & \multirow[t]{3}{*}{4.82} \\
\hline $53.2-1-4,7$ & 3.16 & 0.77 & & $60.0-4-1,145$ & 6.28 & 1.10 & \\
\hline $53.2-1-6,7$ & 2.18 & 0.88 & & $60.0-4-\mathrm{CC}$ & 2.28 & & \\
\hline $54.0-1-1,5$ & 44.80 & 0.20 & 224.00 & $60.0-4-2,145$ & 2.18 & 0.32 & 6.81 \\
\hline $54.0-3-1,140$ & 7.48 & 0.32 & 23.38 & $60.0-5-2,26$ & 3.50 & 0.46 & \\
\hline $54.0-6-1,145$ & 1.48 & 0.18 & 8.22 & $60.0-5-\mathrm{CC}$ & 3.46 & 0.34 & \\
\hline $55.0-3-4,145$ & 54.20 & 0.23 & \multirow[t]{2}{*}{235.65} & $60.0-6-1,86$ & 4.12 & 1.35 & \\
\hline $55.0-4-5,7$ & 53.68 & 0.36 & & $60.0-6-3,14$ & 6.28 & 0.84 & \\
\hline $55.0-9$, Top & 49.28 & 0.26 & \multirow[t]{4}{*}{189.54} & $60.0-6-5,37$ & 2.32 & 0.35 & \\
\hline $55.0-11-3,17$ & 51.92 & 0.27 & & $60.0-6-7,40$ & 2.16 & 0.58 & \\
\hline $55.0-12-3,8$ & 48.90 & 0.54 & & $60.0-7-2,7$ & 3.24 & 0.33 & \\
\hline $55.0-13-1,7$ & 51.20 & 0.27 & & $60.0-8-2,72-73$ & 2.34 & 1.30 & \\
\hline $55.0-13-3,40$ & 52.96 & 0.14 & 378.29 & $60.0-9-2,45$ & 2.12 & 1.22 & \\
\hline
\end{tabular}


TABLE 14

Alkaline Elements as Determined by Flame Photometry

\begin{tabular}{|c|c|c|c|c|c|}
\hline Sample Designation & $\mathrm{Na} \%$ & $\mathrm{~K} \%$ & $\begin{array}{c}\mathrm{Li} \\
\text { n. } 10^{-3} \%\end{array}$ & $\begin{array}{c}\mathrm{Rb} \\
\mathrm{n} \cdot 10^{-3} \%\end{array}$ & $\begin{array}{c}\mathrm{Cs}-\mathrm{HO} \\
\text { n. } 10^{-3} \%\end{array}$ \\
\hline $44.0-1-2,145$ & 0.340 & 0.7000 & & & \\
\hline $44.0-1-1,145$ & 0.650 & 0.0100 & & & \\
\hline $44.0-1-3,50$ & 0.250 & 0.0500 & & 0.00050 & \\
\hline $44.0-1-3,145$ & 0.250 & 0.0600 & & & \\
\hline 44.0-1-3, Mixed & 0.350 & 0.1100 & & & \\
\hline $44.0-1-4,145$ & 0.330 & 0.1500 & & & \\
\hline $44.0-2-\mathrm{CC}$ & 0.200 & & & 0.00080 & \\
\hline $44.0-2-1,145$ & 0.200 & $<0.0500$ & & & \\
\hline $44.0-2-3,145$ & 0.320 & $<0.0500$ & & & \\
\hline $44.0-2-4,145$ & 0.380 & $<0.0500$ & & & \\
\hline $44.0-2-5,145$ & 0.350 & 0.5000 & 0.00010 & $<0.00025 ?$ & \\
\hline $44.0-2-6,145$ & 0.370 & 0.0500 & 0.00010 & 0.00070 & \\
\hline $44.0-3-2,145$ & 0.300 & 0.1900 & & $<0.00025 ?$ & \\
\hline $44.0-3-3,145$ & 0.300 & 0.1000 & & & \\
\hline $44.0-3-4,145$ & 0.270 & 0.0500 & & & \\
\hline $44.0-3-5,145$ & 0.270 & 0.2000 & & & \\
\hline 44.0-4-0, Top & $<0.050$ & 0.7500 & 0.00025 & 0.00300 & \\
\hline $49.0-4-2,145$ & 0.250 & 0.2000 & & & \\
\hline $44.0-4-3,145$ & 0.300 & $<0.0500$ & & & \\
\hline $44.0-4-4,145$ & 0.350 & 0.1100 & & & \\
\hline $44.0-4-5,145$ & 0.370 & 0.0500 & & & \\
\hline $44.0-4-6,145$ & 0.300 & 0.1500 & & & \\
\hline $44-0-4-\mathrm{CC}$ & 0.120 & 0.6200 & & $<0.00050$ & \\
\hline $44.0-5-0$, Total & 0.310 & 0.1000 & & & \\
\hline $44.1-1-\mathrm{CC}$ & 1.500 & 0.9100 & 0.00500 & 0.00500 & \\
\hline $45.1-1-1,7$ & 2.500 & 1.4000 & 0.00490 & 0.00960 & 0.00050 \\
\hline $45.1-1-1,70$ & 1.820 & 0.9000 & 0.00500 & 0.00400 & \\
\hline $45.1-1-1,145$ & 1.600 & 1.2500 & 0.00430 & 0.00590 & \\
\hline $45.1-1-2,100$ & 1.810 & 1.1500 & 0.00480 & 0.00760 & \\
\hline $45.1-1-2,124$ & 1.500 & 0.7000 & 0.00330 & 0.00330 & \\
\hline $45.1-1-3,23$ & 1.500 & 0.9500 & 0.00340 & 0.00450 & \\
\hline $45.1-1-4,22$ & 1.200 & 0.8000 & 0.00290 & 0.00370 & \\
\hline $45.1-1-4,136$ & 0.950 & 0.4800 & 0.00320 & 0.00190 & \\
\hline $45.1-3-\mathrm{CC}$ & 0.050 & 0.2000 & 0.00300 & 0.00470 & \\
\hline $45.1-1-5,4$ & 0.930 & 0.5000 & 0.00290 & 0.00210 & \\
\hline $46.0-1-6$ & 0.140 & 0.3400 & 0.00200 & 0.00360 & \\
\hline $46.0-1-1,145$ & 1.800 & 1.1500 & 0.00530 & 0.01520 & 0.00130 \\
\hline $46.0-1-2,145$ & 1.210 & 1.3500 & 0.00590 & 0.01550 & 0.00120 \\
\hline $46.0-1-3,145$ & 1.920 & 1.1100 & 0.00450 & 0.00900 & \\
\hline $46.0-1-4,145$ & 1.530 & 1.2100 & 0.00500 & 0.01500 & 0.00100 \\
\hline $46.0-1-5,145$ & 1.000 & 1.1000 & 0.00240 & 0.01180 & 0.00080 \\
\hline $46.0-1-6,145$ & 1.980 & 1.2500 & 0.00500 & 0.01180 & 0.00110 \\
\hline $46.1-2-\mathrm{CC}$ & 1.290 & 2.1400 & 0.00200 & 0.00360 & \\
\hline $47.0-1-1,17$ & 0.760 & 0.4900 & & & \\
\hline $47.0-1-2,5$ & 0.600 & 0.3700 & 0.00230 & 0.00640 & 0.00050 \\
\hline $47.0-1-3,16$ & 0.570 & 0.4000 & 0.00200 & 0.00760 & $<0.00050$ \\
\hline $47.0-1-4,90$ & 0.660 & 0.1900 & & & \\
\hline $47.0-1-4,95$ & 1.500 & 1.1300 & 0.00160 & 0.00440 & \\
\hline $47.0-1-5,3.5$ & 0.370 & 0.2000 & 0.00300 & 0.00410 & \\
\hline
\end{tabular}


TABLE 14 - Continued

\begin{tabular}{|c|c|c|c|c|c|}
\hline Sample Designation & $\mathrm{Na} \%$ & $\mathrm{~K} \%$ & $\begin{array}{c}\mathrm{Li} \\
\text { n. } 10^{-3} \%\end{array}$ & $\begin{array}{c}\mathrm{Rb} \\
\text { n. } 10^{-3} \%\end{array}$ & $\begin{array}{l}\mathrm{Cs}-\mathrm{HO} \\
\mathrm{n} .10^{-3} \%\end{array}$ \\
\hline $47.0-1-6,14$ & 0.530 & 0.2200 & & & \\
\hline $47.1-1$, Top of & & & & & \\
\hline Section 1 & 0.370 & 0.2300 & $<0.00050$ & 0.00140 & \\
\hline $47.1-1-1,145$ & 0.500 & 0.1000 & 0.00050 & 0.00220 & \\
\hline $47.1-1-2,16$ & 0.2900 & 0.0600 & 0.00050 & & \\
\hline $47.2-1-2,23$ & 0.7900 & 0.4500 & & & \\
\hline $47.2-1-2,181$ & 0.8000 & 0.3600 & & & \\
\hline $47.2-1-3,11$ & 0.5000 & 0.2700 & 0.00125 & 0.00470 & \\
\hline $47.2-1-3,90$ & 0.5000 & 0.3300 & & & \\
\hline $47.2-1-4,7$ & 0.5000 & 0.2500 & 0.00110 & 0.00720 & \\
\hline $47.2-2-2 \quad 8$ & 0.6000 & 0.3000 & & & \\
\hline $47.2-2-2,47$ & 1.3500 & 0.9400 & 0.00130 & 0.00330 & \\
\hline $47.2-2-3,7$ & 0.4700 & 0.2100 & 0.00070 & 0.00400 & \\
\hline $47,2-2-4,7$ & 0.7000 & 0.2690 & & & \\
\hline $47.2-2-5,5$ & 0.4700 & 0.1900 & 0.00065 & 0.00320 & \\
\hline $47.2-2-6,6$ & 0.5000 & 0.1200 & & & \\
\hline $47.2-3-2,6.5$ & 0.5000 & 0.2500 & 0.00160 & 0.00700 & \\
\hline $47.2-3-3,6$ & 0.7000 & 0.3000 & & & \\
\hline $47.2-3-4,16$ & 0.4000 & 0.1200 & 0.00500 & 0.00170 & \\
\hline $47.2-3-\mathrm{CC}$ & 0.8500 & 0.3300 & 0.00040 & $<0.00050$ & \\
\hline $47.2-4-\mathrm{CC}$ & 1.7000 & 0.2400 & 0.00050 & 0.00100 & \\
\hline $47.2-4-1,16$ & 0.6500 & 0.3200 & & & \\
\hline $47.2-4-2,17$ & 0.4000 & 0.1000 & 0.00025 & 0.00220 & \\
\hline $47.2-4-3,16$ & 0.3000 & 0.0700 & $<0.00050$ & & \\
\hline $47.2-4-4,16.5$ & 0.4000 & 0.1700 & 0.00080 & 0.00250 & \\
\hline $47.2-4-5,16.5$ & 0.3300 & 0.1300 & 0.00050 & & \\
\hline $47.2-4-6,17$ & 0.3300 & 0.2500 & 0.00026 & 0.00240 & \\
\hline $47.2-5-1,17$ & 0.3800 & 0.0900 & $<0.00050$ & & \\
\hline $47.2-5-2,6$ & 0.4000 & $<0.0500$ & 0.00050 & & \\
\hline $47.2-5-3,6$ & 0.3000 & 0.1200 & 0.00010 & 0.00050 & \\
\hline $47.2-5-4,6$ & 0.4000 & 0.1000 & 0.00060 & 0.00100 & \\
\hline $47.2-5-5,6$ & 0.4900 & 0.1400 & & & \\
\hline $47.2-5-6,6.5$ & 0.4000 & 0.1700 & 0.00070 & 0.00200 & \\
\hline $47.2-6-2,6$ & 0.6000 & 0.1900 & & & \\
\hline $47.2-6-3,17$ & 0.4000 & & 0.00065 & 0.00200 & \\
\hline $47.2-7-1,17$ & 0.5000 & 0.3600 & & & \\
\hline $47.2-7-2,17$ & 0.2000 & 0.0500 & & $<0.00050$ & \\
\hline $47.2-7-3,13$ & 0.3500 & 0.1600 & & 0.00083 & \\
\hline $47.2-7-4,17$ & 0.2500 & $<0.1000$ & & 0.00070 & \\
\hline $47.2-7-5,27.5$ & 0.2700 & 0.1000 & & 0.00180 & \\
\hline $47.2-7-6,126$ & 0.3000 & 0.0700 & & 0.00500 & \\
\hline $47.2-8-1,27$ & 0.2300 & $<0.0500$ & & & \\
\hline $47.2-8-2,17$ & 0.2000 & $<0.0500$ & & & \\
\hline $47.2-8-3,18$ & 0.2100 & $<0.0500$ & & & \\
\hline $47.2-8-4,17$ & 0.2500 & 0.0600 & $<0.00050$ & & \\
\hline $47.2-8-5,17$ & 0.2200 & 0.0500 & & & \\
\hline $47.2-8-6,16$ & 0.2200 & 0.0600 & & $<0.0005$ & \\
\hline $47.2-8-6-\mathrm{CC}$ & 0.4500 & 0.0750 & & & \\
\hline $47.2-9-1,16$ & 0.2300 & $<0.0500$ & & & \\
\hline $47.2-9-2,17$ & 0.2200 & 0.0500 & & $<0.0005$ & \\
\hline
\end{tabular}


TABLE 14 - Continued

\begin{tabular}{|c|c|c|c|c|c|}
\hline Sample Designation & $\mathrm{Na} \%$ & $\mathbf{K} \%$ & $\begin{array}{c}\mathrm{Li} \\
\text { n. } 10^{-3} \%\end{array}$ & $\begin{array}{c}\mathrm{Rb} \\
\text { n. } 10^{-3} \%\end{array}$ & $\begin{array}{l}\text { Cs-HO } \\
\text { n. } 10^{-3} \%\end{array}$ \\
\hline $47.2-9-3,17$ & 0.280 & 0.0900 & & 0.00180 & \\
\hline $47.2-9-4,0$ & 0.200 & 0.1000 & & 0.00070 & \\
\hline $47.2-9-5,17$ & 0.210 & 0.0700 & & & \\
\hline $47.2-9-5,150$ & 0.200 & 3.8000 & & 0.00080 & \\
\hline $47.2-9-6,0$ & 0.250 & 0.3000 & & 0.00050 & \\
\hline $47.2-9-\mathrm{CC}$ & 0.200 & 0.1000 & 0.00010 & 0.00090 & \\
\hline $47.2-10-1,17$ & 0.220 & 0.0500 & & & \\
\hline $47.2-10-2,17$ & 0.250 & $<0.0900$ & & 0.00083 & \\
\hline $47.2-10-3,18$ & 0.250 & 0.0500 & & & \\
\hline $47.2-10-4,17$ & 0.270 & 0.0900 & & $<0.00050$ & \\
\hline $47.2-10-5,17$ & 0.300 & 0.0500 & 0.00250 & 0.00060 & \\
\hline $47.2-10-6,16$ & 0.290 & $<0.0500$ & & & \\
\hline $47.2-10-\mathrm{CC}$ & 0.200 & $<0.1000$ & & 0.00060 & \\
\hline 47.2-10-Top & 0.220 & $<0.1000$ & & 0.00050 & \\
\hline $47.2-11-1,145$ & 0.340 & 0.0500 & & & \\
\hline $47.2-11-2,145$ & 0.320 & $<0.0500$ & & & \\
\hline $47.2-11-3,145$ & 0.260 & $<0.1000$ & & $<0.00050$ & \\
\hline $47.2-11-4,145$ & 0.100 & $<0.1000$ & & 0.00060 & \\
\hline $47.2-11-5,145$ & 0.270 & 0.2000 & & & \\
\hline $47.2-11-6,145$ & 0.100 & $<0.1000$ & & 0.00070 & \\
\hline $47.2-11-\mathrm{CC}$ & 0.380 & 0.1300 & & 0.00050 & \\
\hline $47.2-12-1,145$ & 0.580 & 2.3700 & & $<0.00050$ & \\
\hline $47.2-12-2,17$ & 0.210 & 0.1100 & & 0.00075 & \\
\hline $47.2-12-4,145$ & 0.280 & $<0.0500$ & & & \\
\hline $47.2-12-\mathrm{CC}$ & 0.600 & 0.2000 & & $<0.00050$ & \\
\hline $47.2-13-1,145$ & 0.470 & 0.1600 & & 0.00050 & \\
\hline $47.2-13-2,145$ & 0.260 & 0.0300 & & & \\
\hline $47.2-13-3,16$ & 0.250 & $<0.0500$ & & & \\
\hline $47.2-13-4,145$ & 0.250 & 0.1000 & $<0.0005$ & & \\
\hline $47.2-13-5,145$ & 0.250 & $<0.1000$ & & $<0.00040$ & \\
\hline $47.2-13-6,145$ & 0.230 & $<0.0500$ & & & \\
\hline $47.2-13-\mathrm{CC}$ & 0.550 & 0.1300 & & & \\
\hline $47.2-14-1,145$ & 0.300 & $<0.1000$ & & 0.00050 & \\
\hline $47.2-14-2,145$ & 0.220 & $<0.0500$ & & $<0.00050$ & \\
\hline $47.2-14-3,145$ & 0.300 & $<0.1000$ & & & \\
\hline $47.2-14-4,145$ & 0.240 & $<0.0500$ & & & \\
\hline $47.2-14-5,145$ & 0.270 & 0.0500 & & $<0.00050$ & \\
\hline $47.2-14-6,145$ & 0.240 & 0.0900 & & 0.00180 & \\
\hline $47.2-14-\mathrm{CC}$ & 0.550 & 0.4500 & & $<0.00050$ & \\
\hline $48.1-1-1.26$ & 0.450 & 0.2700 & 0.00080 & 0.00400 & \\
\hline $48.2-1-\mathrm{CC}$ & 1.020 & 0.1300 & 0.00030 & 0.00050 & \\
\hline $48.2-1-1,28$ & 0.400 & $<0.1000$ & 0.00050 & 0.00016 & \\
\hline $48.2-1-2,33$ & 0.550 & 0.0900 & & & \\
\hline $48.2-1-3,16$ & 0.500 & 0.2500 & 0.00075 & 0.00250 & \\
\hline $48.2-1-4,16$ & 0.600 & 0.1900 & & & \\
\hline $48.2-1-5,14.5$ & 0.510 & 0.1700 & 0.00090 & 0.00250 & \\
\hline $48.2-1-6,11$ & 0.270 & 0.0200 & & & \\
\hline $48.2-2-1,145$ & 0.300 & $<0.1000$ & & 0.00140 & \\
\hline $48.2-2-2,17$ & 0.250 & 0.1000 & & & \\
\hline
\end{tabular}


TABLE 14 - Continued

\begin{tabular}{|c|c|c|c|c|c|}
\hline Sample Designation & $\mathrm{Na} \%$ & $\mathrm{~K} \%$ & $\begin{array}{c}\mathrm{Li} \\
\text { n. } 10^{-3} \%\end{array}$ & $\begin{array}{c}\mathrm{Rb} \\
\text { n. } 10^{-3} \%\end{array}$ & $\begin{array}{l}\mathrm{Cs}-\mathrm{HO} \\
\text { n. } 10^{-3} \%\end{array}$ \\
\hline $48.2-2-3,145$ & 0.270 & 0.0200 & & & \\
\hline $48.2-2-4,18$ & 0.300 & $<0.0500$ & & & \\
\hline $48.2-2-5,16$ & 0.280 & 0.0300 & & & \\
\hline $48.2-2-6,16$ & 0.400 & 0.3000 & & $<0.00050$ & \\
\hline $48.2-2-\mathrm{CC}$ & 0.540 & 0.0075 & & 0.00030 & \\
\hline $48.2-3-1,117$ & 0.290 & 0.0200 & & & \\
\hline $48.2-3-2,28$ & 0.250 & $<0.0500$ & & & \\
\hline $48.2-3-3,17$ & 0.230 & 0.0300 & & & \\
\hline $48.2-3-4,17$ & 0.260 & 0.0700 & & & \\
\hline $48.2-3-5,16$ & 0.250 & 0.0200 & & & \\
\hline $49.0-1-1,76$ & 0.770 & 0.5700 & 0.00320 & 0.01720 & 0.00140 \\
\hline $49.0-1-3,16$ & 1.600 & 1.2000 & 0.00670 & 0.02200 & 0.00220 \\
\hline $49.0-1-5,7$ & 1.000 & 0.8300 & 0.00600 & 0.02100 & 0.00200 \\
\hline $49.0-1-5,130$ & $<0.050$ & 0.0500 & 0.00070 & 0.00350 & \\
\hline $49.0-1-6,7$ & 1.700 & 0.8200 & 0.00700 & 0.01830 & 0.00190 \\
\hline $49.1-1-2,16$ & 1.200 & 0.2700 & 0.00700 & 0.01100 & 0.00180 \\
\hline $49.1-1-3,22$ & 1.100 & 1.2500 & $<0.00400$ & 0.01300 & 0.00110 \\
\hline $49.1-1-4,117$ & 0.470 & 0.3700 & 0.00260 & 0.00550 & $<0.00050$ \\
\hline $49.1-1-5,60$ & 1.500 & 0.9600 & 0.00080 & 0.00280 & \\
\hline $49.1-2-\mathrm{CC}$ & $<0.050$ & 0.1000 & 0.00110 & 0.00390 & \\
\hline $49.1-2-1,145$ & 0.300 & 0.1600 & $<0.00050$ & 0.00130 & \\
\hline $49.1-2-3,145$ & 0.370 & 0.2200 & 0.00090 & 0.00370 & \\
\hline $50.0-2-\mathrm{CC}$ & $<0.050$ & $<0.0500$ & 0.00080 & 0.00310 & \\
\hline $50.0-2-1,145$ & 0.420 & 0.1500 & 0.00110 & 0.00100 & \\
\hline $50.0-2-2,150$ & 0.430 & 0.1700 & 0.00060 & 0.00350 & \\
\hline $50.2-3,150$ & 1.650 & 0.9600 & 0.00120 & 0.00280 & \\
\hline $50.0-2-4,150$ & 0.850 & $<0.1000$ & 0.00080 & 0.00220 & \\
\hline $50.0-2-6,150$ & 0.340 & 0.1700 & 0.00060 & 0.00300 & \\
\hline $50.1-1-3,7$ & 0.870 & 0.9200 & 0.00390 & 0.01550 & 0.00170 \\
\hline $50.1-1-5,15$ & 0.720 & 0.5700 & 0.00320 & 0.00900 & 0.00090 \\
\hline $50.1-2-1,130$ & 0.850 & 0.5700 & 0.00420 & 0.01120 & 0.00050 \\
\hline $50.1-2-3,35$ & 1.440 & 1.0300 & 0.00500 & 0.01300 & 0.00120 \\
\hline $50.1-2-4,50$ & 2.340 & 1.1900 & 0.01090 & 0.01390 & 0.00053 \\
\hline $50.1-2-5,8$ & 1.270 & 1.0200 & 0.00750 & 0.01580 & 0.00050 \\
\hline $50.1-3-3,15$ & 1.100 & 0.9000 & 0.00560 & 0.01450 & 0.00130 \\
\hline $50.1-3-5,28$ & 0.970 & 1.2500 & 0.00700 & 0.01730 & 0.00130 \\
\hline $51.0-1-1,11$ & 1.300 & 1.0500 & 0.00700 & 0.01750 & 0.00150 \\
\hline $51.0-1-3,6$ & 1.400 & 0.3000 & 0.00570 & 0.01430 & 0.00160 \\
\hline $51.0-1-5,15$ & 0.800 & 1.2000 & 0.00700 & 0.01270 & 0.00100 \\
\hline $51.0-2-\mathrm{CC}$ & 3.860 & 1.4000 & 0.00460 & 0.01210 & 0.00080 \\
\hline $51.1-1,145$ & 0.750 & 1.2500 & 0.00530 & 0.01080 & 0.00080 \\
\hline $51.1-1-1,15$ & 1.250 & 1.1700 & 0.00610 & 0.01000 & \\
\hline $51.1-1-3,30$ & 0.920 & 0.9200 & 0.00520 & 0.00710 & 0.00050 \\
\hline $51.1-1-4,30$ & 2.720 & 1.7200 & 5.05000 & 12.10000 & 0.00010 \\
\hline $51.1-1-5,16$ & 0.910 & 0.9700 & 0.00400 & 0.01030 & 0.00100 \\
\hline $51.1-2-1,127$ & 0.810 & 1.1700 & 0.00600 & 0.01100 & 0.00100 \\
\hline $52.0-1-1,27$ & 1.110 & 1.0200 & 0.00550 & 0.01470 & 0.00110 \\
\hline $52.0-1-3,8$ & 0.950 & 0.9700 & 0.00600 & 0.01430 & 0.00125 \\
\hline $52.0-1-5,10$ & 1.000 & 0.7200 & 0.00530 & 0.00690 & $<0.00050$ \\
\hline
\end{tabular}


TABLE 14 - Continued

\begin{tabular}{|c|c|c|c|c|c|}
\hline Sample Designation & $\mathrm{Na} \%$ & $\mathrm{~K} \%$ & $\begin{array}{c}\mathrm{Li} \\
\text { n. } 10^{-3} \%\end{array}$ & $\begin{array}{c}\mathrm{Rb} \\
\mathrm{n} .10^{-3} \%\end{array}$ & $\begin{array}{l}\text { Cs-HO } \\
\text { n. } 10^{-3} \%\end{array}$ \\
\hline $52.0-2-\mathrm{CC}$ & 1.620 & 1.2400 & 0.00520 & 0.00100 & \\
\hline $52.0-2-2,7$ & 1.020 & 1.0200 & 0.00560 & 0.01620 & 0.00230 \\
\hline $52.0-2-4,8$ & 1.350 & 1.3500 & 0.00480 & 0.00690 & 0.00050 \\
\hline $52.0-3-2,19$ & 1.000 & 0.8700 & 0.00620 & 0.01000 & 0.00070 \\
\hline $52.0-3-4,16$ & 1.000 & 0.7500 & 0.00950 & 0.00600 & $<0.00050$ \\
\hline $52.0-3-6,6$ & 0.600 & 0.6500 & 0.00650 & 0.00360 & \\
\hline $52.0-4-3,5$ & 1.080 & 0.8000 & 0.00650 & 0.01480 & 0.00130 \\
\hline $52.0-4-5,6$ & 1.200 & 0.9000 & 0.00550 & 0.01040 & 0.00070 \\
\hline $52.0-4-6,128$ & 0.920 & 0.7000 & 0.00580 & 0.01150 & 0.00100 \\
\hline $52.0-5-2,14$ & 1.470 & 0.6500 & 0.00580 & 0.01200 & 0.00180 \\
\hline $52.0-5-3,15$ & 1.400 & 0.7800 & 0.00640 & 0.00840 & 0.00070 \\
\hline $52.0-5-4,18$ & 1.100 & 0.9200 & 0.00560 & 0.00670 & 0.00050 \\
\hline $52.0-6-\mathrm{CC}$ & 1.000 & 1.2500 & 0.00490 & 0.00360 & \\
\hline $52.0-6-1,24$ & 1.100 & 1.0000 & 0.00670 & 0.01210 & 0.00080 \\
\hline $52.0-7-\mathrm{CC}$ & 0.500 & 1.3300 & 0.00100 & 0.00200 & \\
\hline $52.0-8-1,145$ & 0.920 & 0.7200 & 0.00420 & 0.00900 & 0.00100 \\
\hline $52.0-8-2,145$ & 1.300 & 0.9000 & 0.00320 & 0.00950 & 0.00050 \\
\hline $52.0-8-3,21$ & 1.400 & 0.9000 & 0.00430 & 0.01090 & 0.00070 \\
\hline $52.0-8-3,40$ & 1.000 & 0.8100 & 0.00250 & 0.00680 & 0.00050 \\
\hline $52.0-8-4,145$ & 0.750 & 0.5200 & 0.00260 & 0.00980 & 0.00100 \\
\hline $52.0-8-5,145$ & 0.960 & 0.7100 & 0.00230 & 0.00660 & 0.00050 \\
\hline $52.0-8-6,145$ & 1.200 & 1.0000 & 0.00380 & 0.01050 & 0.00090 \\
\hline $52.0-10-\mathrm{CC}$ & 1.000 & 1.5100 & 0.00580 & 0.01700 & 0.00140 \\
\hline $53.0-1-2,144$ & 1.150 & 0.8500 & 0.00230 & 0.00360 & \\
\hline $53.0-2-\mathrm{CC}$ & 1.050 & 0.5000 & 0.00300 & 0.00230 & \\
\hline $53.0-3-1,17$ & 0.900 & 0.3100 & 0.00260 & 0.00200 & \\
\hline $53.0-4-2,0$ & 1.130 & 1.5500 & 0.00340 & 0.00460 & \\
\hline $53.0-6-2,65$ & 0.150 & 0.3700 & $<0.00050$ & 0.00080 & \\
\hline $53.0-6-\mathrm{CC}$ & 0.500 & 0.7300 & 0.00160 & 0.00075 & \\
\hline $53.1-1-2,7$ & 1.400 & 0.7200 & 0.00540 & 0.00590 & \\
\hline $53.1-1-3,18$ & 1.250 & 0.6500 & 0.00480 & 0.00690 & 0.00050 \\
\hline $53.1-1-5,18$ & 1.200 & 0.2500 & 0.00230 & 0.00200 & \\
\hline $53.1-2-1,145$ & 1.350 & 0.7200 & 0.00110 & 0.00570 & \\
\hline $53.1-2-2,145$ & 1.100 & 2.6000 & 0.00080 & 0.00360 & \\
\hline $53.1-2-3,90$ & 1.120 & 0.6500 & 0.00130 & 0.00400 & \\
\hline $53.1-2-5,7$ & 1.400 & 0.7200 & 0.00150 & 0.00450 & \\
\hline $53.1-3-1,150$ & 1.300 & 0.6500 & 0.00200 & 0.00190 & \\
\hline $53.1-3-2,16$ & 1.100 & 0.5500 & 0.00130 & 0.00340 & \\
\hline $53.1-3-4,26$ & 1.120 & 0.6500 & 0.00120 & 0.00370 & \\
\hline $53.2-1-2,16$ & 1.100 & 0.5200 & 0.00175 & 0.00280 & \\
\hline $53.2-1-4,7$ & 0.800 & 0.5000 & 0.00120 & 0.00170 & \\
\hline $53.2-1-6,7$ & 1.100 & 0.6000 & 0.00130 & 0.00340 & \\
\hline $53.2-4-\mathrm{CC}$ & 1.040 & 0.4600 & 0.00010 & 0.00020 & \\
\hline $53.2-5-\mathrm{CC}$ & 1.140 & 0.8500 & 0.00090 & 0.00100 & \\
\hline $54.0-1-1,5$ & 0.300 & 0.1800 & 0.00070 & 0.00230 & \\
\hline $54.0-1-2,7$ & 1.150 & 0.7200 & 0.00180 & 0.00390 & \\
\hline $54.0-1-2,20$ & 1.050 & 0.6500 & 0.00180 & 0.00440 & \\
\hline $54.0-2-1,17$ & 0.970 & 0.4200 & 0.00110 & 0.00230 & \\
\hline $54.0-2-4,16$ & 0.850 & 0.5000 & 0.00110 & 0.00370 & $<0.00050$ \\
\hline
\end{tabular}


TABLE 14 - Continued

\begin{tabular}{|c|c|c|c|c|c|}
\hline Sample Designation & $\mathrm{Na} \%$ & $\mathrm{~K} \%$ & $\begin{array}{c}\mathrm{Li} \\
\text { n. } 10^{-3} \%\end{array}$ & $\begin{array}{c}\mathrm{Rb} \\
\mathrm{n} \cdot 10^{-3} \%\end{array}$ & $\begin{array}{l}\text { Cs-HO } \\
\text { n. } 10^{-3} \%\end{array}$ \\
\hline $54.0-4-2,14$ & 1.100 & 0.6100 & 0.00150 & 0.00300 & \\
\hline $54.0-6-3,23$ & 1.090 & 0.6200 & 0.00170 & 0.00260 & \\
\hline $54.0-6-4,78$ & 1.000 & 0.5500 & 0.00240 & 0.00300 & 0.00050 \\
\hline $54.0-7-1,138$ & 1.100 & 0.6800 & 0.00120 & 0.00270 & \\
\hline $54.0-7-3,16$ & 1.100 & 0.6700 & 0.00120 & 0.00280 & $<0.00050$ \\
\hline $54.0-9-1$ & 0.350 & 0.1000 & 0.00100 & 0.00050 & \\
\hline $55.0-1-2,7$ & 0.700 & $<0.1000$ & 0.00025 & 0.00080 & \\
\hline $55.0-1-4,7$ & 0.400 & $>2.0000$ & 0.00060 & 0.00060 & \\
\hline $55.0-1-6,7$ & 0.380 & 0.0500 & 0.00050 & 0.00090 & \\
\hline $55.0-2-1,7$ & 0.032 & 0.0030 & & & \\
\hline $55.0-2-2,7$ & 0.340 & 0.1100 & $<0.00050$ & $<0.00050$ & \\
\hline $55.0-2-3,16$ & 0.037 & 0.0080 & & & \\
\hline $55.0-2-3-\mathrm{CC}$ & 1.540 & 0.7200 & 0.00050 & 0.00040 & \\
\hline $55.0-2-4,15$ & 0.340 & 0.0600 & $<0.00050$ & & \\
\hline $55,0-2-5,70$ & 0.420 & 0.2200 & $<0.00050$ & 0.00050 & \\
\hline $55.0-3-1,16$ & 0.350 & 0.2000 & $<0.00050$ & $<0.00050$ & \\
\hline $55.0-3-3,14$ & 0.036 & 0.0060 & & & \\
\hline $55.0-3-4,145$ & 0.570 & 0.0750 & & 0.00040 & \\
\hline $55.0-3-5,15$ & 0.027 & 0.0050 & & & \\
\hline $55.0-4-1,13$ & 0.290 & 0.0700 & $<0.00050$ & & \\
\hline $55.0-4-2,145$ & 0.026 & $<0.0040$ & & & \\
\hline $55.0-4-3,7$ & 0.320 & 0.2700 & $<0.00050$ & & \\
\hline $55.0-4-4,145$ & 0.002 & & & & \\
\hline $55.0-4-5,7$ & 0.270 & $<0.0500$ & & $<0.00025$ & \\
\hline $55.0-4-6,145$ & 0.020 & 0.0030 & & & \\
\hline $55.0-5-1,7$ & 0.500 & 0.2700 & $<0.00050$ & & \\
\hline $55.0-5-5,7$ & 0.320 & 0.1900 & $<0.00050$ & $<0.00050$ & \\
\hline $55.0-6-3,17$ & 0.500 & 0.6700 & 0.00050 & 0.00050 & \\
\hline $55.0-6-5,16$ & 0.025 & 0.0040 & & & \\
\hline $55.0-7-1,14$ & 0.540 & 0.6100 & $<0.00050$ & & \\
\hline $55.0-7-3,18$ & 0.038 & 0.0090 & & & \\
\hline $55.0-7-5,19$ & 0.320 & 0.0500 & $<0.00050$ & & \\
\hline $55.0-8-3,16$ & 0.500 & 0.7000 & 0.00025 & $<0.00050$ & \\
\hline $55.0-8-5,18$ & 0.032 & 0.0070 & & & \\
\hline $55.0-9$ & 0.600 & 0.1500 & 0.00025 & 0.00040 & \\
\hline $55.0-10-2,10$ & 0.370 & 0.0500 & 0.00050 & 0.00040 & \\
\hline $55.0-10-5,16$ & 0.440 & 0.2700 & $<0.00050$ & $<0.00050$ & \\
\hline $55,0-11-3,17$ & 0.400 & $<0.0500$ & 0.00050 & 0.00080 & \\
\hline $55.0-11-5,7$ & 0.082 & 0.0150 & & & \\
\hline $55.0-12-1,13$ & 0.320 & 0.0500 & $<0.00025$ & & \\
\hline $55.0-12-2,13$ & 0.024 & 0.0055 & & & \\
\hline $55.0-12-3,8$ & 0.270 & 0.0500 & $<0.00050$ & & \\
\hline $55.0-13-1,7$ & 0.290 & 0.1700 & $<0.00050$ & 0.00050 & \\
\hline $55.0-13-3,7$ & 0.023 & 0.0050 & & & \\
\hline $55.0-13-3,40$ & 0.870 & 0.1700 & 0.00025 & & \\
\hline $55.0-13-5,7$ & 0.270 & 0.1500 & $<0.00050$ & 0.00050 & \\
\hline $55.0-14-1,145$ & 0.018 & 0.0023 & & & \\
\hline $55.0-14-2,145$ & 0.260 & 0.1200 & $<0.00050$ & 0.00150 & \\
\hline $55.0-14-3,145$ & 0.019 & 0.0030 & & & \\
\hline
\end{tabular}


TABLE 14 - Continued

\begin{tabular}{|c|c|c|c|c|c|}
\hline Sample Designation & $\mathrm{Na} \%$ & $\mathrm{~K} \%$ & $\begin{array}{c}\mathrm{Li} \\
\mathrm{n} .10^{-3} \%\end{array}$ & $\begin{array}{c}\mathrm{Rb} \\
\mathrm{n} .10^{-3} \%\end{array}$ & $\begin{array}{l}\mathrm{Cs}-\mathrm{HO} \\
\text { n. } 10^{-3} \%\end{array}$ \\
\hline $55.0-14-3,145$ & 0.019 & 0.0030 & & & \\
\hline $55.0-14-4,145$ & 0.270 & $<0.0500$ & $<0.00025$ & & \\
\hline $55.0-14-6,145$ & 0.270 & 0.0900 & $<0.00500$ & $<0.00050$ & \\
\hline $56.2-1-2,145$ & 0.200 & $<0.0500$ & & & \\
\hline $56.2-1-6,7$ & 0.220 & 0.0700 & & & \\
\hline $56.2-2-4,36$ & 0.400 & 0.4700 & & & \\
\hline $56.2-3-2,145$ & 0.290 & $<0.0500$ & & & \\
\hline $56.2-3-6,145$ & 0.270 & 0.0500 & $<0.00025$ & & \\
\hline $56.2-4-4,14$ & 0.290 & $<0.0500$ & $<0.00025$ & & \\
\hline $56.2-5-2,13$ & 0.270 & $<0.0500$ & 0.00030 & $<0.00025$ & \\
\hline $56.2-5-6,7$ & 0.350 & 0.1900 & $<0.00050$ & & \\
\hline $56.2-6-2,7$ & 0.250 & $<0.0500$ & 0.00030 & $<0.00025$ & \\
\hline $56.2-7-6,7$ & 0.200 & $<0.0500$ & $<0.00025$ & & \\
\hline $56.2-8-4,7$ & 0.290 & 0.0500 & $<0.00050$ & & \\
\hline $56.2-10-2,10$ & 0.450 & 0.1400 & $<0.00050$ & 1.10000 & \\
\hline $56.2-10-6,13$ & 0.650 & 0.5000 & 0.00150 & 0.00250 & \\
\hline $57.0-1-1,5$ & 0.550 & 0.1900 & & & \\
\hline 57.0 & $0.003 ?$ & $0.0040 ?$ & & & \\
\hline $57.1-1-1,10$ & 0.450 & 0.1200 & $<0.00050$ & $<0.00050$ & \\
\hline $57.1-1-2,13$ & 1.200 & 0.9000 & $<0.00050$ & & \\
\hline $57.1-1-4,7$ & 0.310 & 0.1000 & $<0.00050$ & & \\
\hline $57.1-1-6,11$ & 0.360 & & $<0.00050$ & $<0.00050$ & \\
\hline $57.1-3-1,22$ & 0.600 & $<0.1000$ & 0.00030 & 0.00080 & \\
\hline $57.1-4-1,86$ & 0.450 & 0.0500 & 0.00100 & & 0.00080 \\
\hline $57.1-4-3,14$ & 0.370 & 0.0800 & 0.00060 & 0.00060 & \\
\hline $57.1-4-4,145$ & 1.100 & 0.3700 & 0.00073 & 0.00070 & \\
\hline $58.1-1-1,139$ & 0.520 & $<0.0500$ & $<0.00050$ & 0.00060 & \\
\hline $58.1-1-2,91$ & 0.370 & 0.2500 & $<0.00050$ & 1.10000 & \\
\hline $58.2-1-2,16$ & 0.630 & 0.2000 & 0.00175 & 0.00190 & \\
\hline $58.2-1-4,31$ & 1.100 & 0.2500 & 0.00340 & 0.00180 & \\
\hline $58.2-1-5,1$ & 0.800 & 0.1700 & 0.00330 & 0.00110 & \\
\hline $59.1-3-1,130$ & 1.350 & 0.6200 & 0.00630 & 0.00950 & 0.00100 \\
\hline $59.1-3-\mathrm{CC}$ & 1.820 & 1.2500 & 0.00240 & 0.00880 & 0.00025 \\
\hline $59.2-1-1,47$ & 1.750 & 0.5700 & 0.00480 & 0.00200 & \\
\hline $59.2-1-3,4$ & 1.520 & 0.4200 & 0.00500 & 0.00190 & \\
\hline $59.2-2-2,145$ & 1.500 & 0.4000 & 0.00470 & 0.00330 & \\
\hline $59.2-2-4,4$ & 1.800 & 0.4500 & 0.00500 & 0.00250 & \\
\hline $59.2-2-6,16$ & $>2.000$ & 0.5000 & 0.00430 & 0.00250 & \\
\hline $59.2-2-\mathrm{CC}$ & 1.910 & 0.5000 & 0.00400 & 0.00160 & \\
\hline $59.2-3$, Top of core & 1.020 & 0.5100 & 0.00500 & 0.00280 & \\
\hline $59.2-3-1,145$ & 1.420 & 0.5700 & 0.00610 & 0.00350 & \\
\hline $59.2-3-\mathrm{CC}$ & 2.850 & 3.2600 & 0.00520 & 0.00360 & \\
\hline $59.2-4-1,66$ & 1.600 & 0.7000 & 0.00450 & 0.00270 & $<0.00050$ \\
\hline $59.2-4-\mathrm{CC}$ & 3.710 & 1.9900 & 0.00390 & 0.00240 & \\
\hline $59.2-5-\mathrm{CC}$ & 1.800 & 1.2000 & 0.00490 & 0.00450 & \\
\hline $59.2-6$ Top of core & 0.700 & 0.4300 & 0.00400 & 0.00130 & \\
\hline $59.2-6-2,0$ & 1.900 & 0.5000 & 0.00600 & 0.00350 & $<0.00050$ \\
\hline $60.0-1-2,9$ & 0.470 & 0.2000 & 0.00125 & & \\
\hline $60.0-1-3,10$ & 0.570 & 0.3100 & 0.00125 & $<0.00050$ & \\
\hline
\end{tabular}


TABLE 14 - Continued

\begin{tabular}{|c|c|c|c|c|c|}
\hline Sample Designation & $\mathrm{Na} \%$ & $\mathrm{~K} \%$ & $\begin{array}{c}\mathrm{Li} \\
\text { n. } 10^{-3} \%\end{array}$ & $\begin{array}{c}\mathrm{Rb} \\
\text { n. } 10^{-3} \%\end{array}$ & $\begin{array}{l}\text { Cs-HO } \\
\text { n. } 10^{-3} \%\end{array}$ \\
\hline $60.0-2-2,12$ & 0.950 & 0.5500 & 0.00210 & 0.00340 & \\
\hline $60.0-3-1,117$ & 0.800 & 0.5200 & 0.00090 & 0.00400 & 0.00050 \\
\hline $60.0-3-\mathrm{CC}$ & 1.800 & 1.2500 & 0.00170 & 0.00170 & \\
\hline $60.0-4-\mathrm{CC}$ & 1.210 & 0.8000 & 0.00130 & 0.00360 & \\
\hline $60.0-4-1,145$ & 2.560 & 1.8500 & 0.00120 & 0.00250 & \\
\hline $60.0-4-2,145$ & 1.570 & 1.1100 & 0.00100 & 0.00480 & \\
\hline $60.0-5-2,26$ & 1.100 & 0.7200 & 0.00125 & 0.00370 & \\
\hline $60.0-5-\mathrm{CC}$ & 1.390 & 0.7500 & 0.00180 & 0.00360 & \\
\hline $60.0-6-1,86$ & 1.220 & 0.4000 & 0.00240 & 0.00450 & $<0.00050$ \\
\hline $60.0-6-3,14$ & 0.970 & 0.4200 & 0.00200 & 0.00360 & \\
\hline $60.0-6-5,37$ & 1.350 & 0.5500 & 0.00125 & 0.00260 & \\
\hline $60.0-6-7,40$ & 1.070 & 0.6000 & 0.00110 & 0.00380 & 0.00050 \\
\hline $60.0-7-2,7$ & 1.050 & 0.5000 & 0.00080 & 0.00430 & \\
\hline $60.0-8-1,145$ & 1.010 & 0.5900 & & & \\
\hline $60.0-8-2,72$ & 0.900 & 0.4700 & 0.00240 & 0.00250 & 0.00050 \\
\hline $60.0-9-2,145$ & 0.750 & 0.4000 & 0.00200 & 0.00270 & $<0.00050$ \\
\hline $60.0-9-5,16$ & 1.040 & 0.3800 & & & \\
\hline
\end{tabular}


TABLE 15

Determination of $\mathrm{Zn}, \mathrm{Cu}, \mathrm{Ni}, \mathrm{Cd}, \mathrm{Sr}$ by Atomic Absorption

\begin{tabular}{|c|c|c|c|c|c|c|c|c|c|c|c|}
\hline Sample Designation & $\mathrm{Zn}$ & $\mathrm{Cu}$ & $\mathrm{Ni}$ & $\mathrm{Cd}$ & $\mathrm{Sr}$ & Sample Designation & $\mathrm{Zn}$ & $\mathrm{Cu}$ & $\mathrm{Ni}$ & $\mathrm{Cd}$ & $\mathrm{Sr}$ \\
\hline $44.0-1-1,145$ & 430 & & & 0.085 & & $46.0-1-1,145$ & 840 & & & 0.044 & \\
\hline $44.0-1-1,145$ & 675 & & & 0.150 & & $46.0-1-2,145$ & 365 & 188 & 153 & & 20 \\
\hline $44.0-1-3,50$ & 43 & & & 0.059 & 1850 & $46.0-1-3,145$ & 440 & 180 & 135 & & 45 \\
\hline $44.0-1-3,145$ & 550 & & & 0.230 & & $46.0-1-4,145$ & 645 & 257 & 230 & & $<10$ \\
\hline $44.0-1-3$, mixed & 400 & & & 0.370 & & $46.0-1-5,145$ & 382 & 138 & 97 & 0.011 & \\
\hline $44.0-1-4,145$ & 965 & & & 0.190 & & $46.0-1-6,145$ & 1030 & 195 & 142 & & 35 \\
\hline $44.0-2-\mathrm{CC}$ & 80 & & & 0.400 & 2950 & $46.1-2-\mathrm{CC}$ & 160 & 165 & & 0.030 & \\
\hline $44.0-2-1,145$ & 30 & & & 0.100 & 1850 & $47.0-1-1,17$ & 55 & & & 0.055 & 1000 \\
\hline $44.0-2-2,145$ & & & & 0.160 & & $47.0-1-2,5$ & & & & 0.034 & \\
\hline $44.0-2-3,145$ & 28 & & & 0.120 & & $47.0-1-3,16$ & 60 & & & 0.080 & \\
\hline $44.0-2-4,145$ & 30 & & & 0.115 & 2250 & $47.0-1-4,90$ & 55 & & & 0.068 & 1200 \\
\hline $44.0-2-5,145$ & 30 & & & 0.050 & & $47.0-1-4,95$ & 50 & 83 & & 0.115 & 1600 \\
\hline $44.0-2-6,145$ & 700 & & & 0.290 & & $47.0-1-5,3.5$ & 37 & & & 0.056 & 2300 \\
\hline $44.0-3-\mathrm{CC}$ & 5840 & 8800 & & 0.540 & & $47.0-1-6,14$ & 38 & & & 0.058 & 1200 \\
\hline $44.0-3-2,145$ & 40 & & & 0.092 & & $47.1-1$, Top of & & & & & \\
\hline $44.0-3-3,145$ & 60 & & & 0.047 & & Section 1 & 365 & & & 0.150 & \\
\hline $44.0-3-4,145$ & 37 & & & 0.110 & & $47.1-1-1,145$ & 1350 & & & & \\
\hline $44.0-3-5,145$ & 465 & & & 0.020 & & $47.1-1-2,16$ & 195 & & & 0.100 & \\
\hline $44.0-4-0$, Top & 90 & & & 0.010 & & $47.1-3-1,17$ & 405 & & & 0.084 & \\
\hline $44.0-4-2,145$ & 102 & & & 0.016 & & $47.2-1-2,23$ & 42 & & & 0.076 & 1050 \\
\hline $44.0-4-3,145$ & 37 & & & 0.021 & & $47.2-1-2,181$ & 79 & & & 0.055 & 1250 \\
\hline $44.0-4-4,145$ & 34 & & & 0.027 & & $47.2-1-3,11$ & 65 & & & 0.120 & 2100 \\
\hline $44.0-4-5,145$ & 39 & & & 0.084 & & $47.2-1-3,90$ & 41 & & & 0.060 & 1350 \\
\hline $44.0-4-6,145$ & 110 & & & 0.110 & & $47.2-1-4,7$ & 88 & & & 0.033 & 2200 \\
\hline $44.0-4-\mathrm{CC}$ & 238 & 15 & & 0.095 & 2000 & $47.2-2-2,8$ & 155 & & & 0.046 & 900 \\
\hline $44.0-5-0$, Total & 2000 & & & 0.240 & & $47.2-2-2,47$ & 73 & 37 & 50 & 0.052 & 1400 \\
\hline 44.1-1-CC & 206 & 224 & & $<0.010$ & 20 & $47.2-2-3,7$ & 60 & & & 0.020 & 2650 \\
\hline $45.1-1-1,7$ & 325 & & & 0.012 & 50 & $47.2-2-4,7$ & 42 & & & 0.045 & 1200 \\
\hline $45.1-1-1,70$ & 160 & 290 & & $<0.010$ & $<20$ & $47.2-2-5,5$ & 50 & & & 0.088 & 2750 \\
\hline $45.1-1-1,145$ & 400 & & & 0.011 & 35 & $47.2-2-6,6$ & 32 & & & 0.070 & 1450 \\
\hline $45.1-1-2,100$ & 480 & & & $<0.010$ & 50 & $47.2-3-2,6.5$ & 60 & & & 0.180 & 2200 \\
\hline $45.1-1-2,124$ & 172 & & & 0.012 & 70 & $47.2-3-3,6$ & 45 & & & 0.068 & 2200 \\
\hline $45.1-1-3,23$ & 154 & & & 0.010 & 175 & $47.2-3-4,16$ & 35 & & & 0.041 & 2750 \\
\hline $45.1-1-4,22$ & 220 & & & 0.010 & 80 & $47.2-3-\mathrm{CC}$ & 167 & 28 & & 0.026 & 3100 \\
\hline $45.1-1-4,136$ & 107 & & & 0.140 & 170 & $47.2-4-\mathrm{CC}$ & 378 & 30 & & 0.145 & 2900 \\
\hline $45.1-2-\mathrm{CC}$ & 130 & 180 & 208 & 0.050 & 70 & $47.2-4-1,16$ & 28 & & & 0.085 & 2150 \\
\hline $45.1-3-\mathrm{CC}$ & 330 & & & 0.077 & 40 & $47.2-4-2,17$ & 32 & & & 0.135 & 2800 \\
\hline $45.1-3-\mathrm{CC}$ & & & & 0.075 & & $47.2-4-3,16$ & 17 & & & 0.060 & 3400 \\
\hline $45.1-1-5,4$ & 103 & & & 0.300 & & $47.2-4-4,16.5$ & 37 & & & 0.020 & 2600 \\
\hline $46.0-$ & 40 & 12 & & $<0.070$ & 70 & $47.2-4-5,16.5$ & 22 & & & 0.080 & 3200 \\
\hline $46.0-1-6$ & 120 & 38 & 27 & 0.021 & 40 & $47.2-4-6,17$ & 45 & & & 0.080 & 2700 \\
\hline $46.0-1-6$ & 40 & 28 & $<10$ & $<0.010$ & $<20$ & $47.2-5-1,17$ & 17 & & & 0.021 & 3200 \\
\hline
\end{tabular}


TABLE 15 - Continued

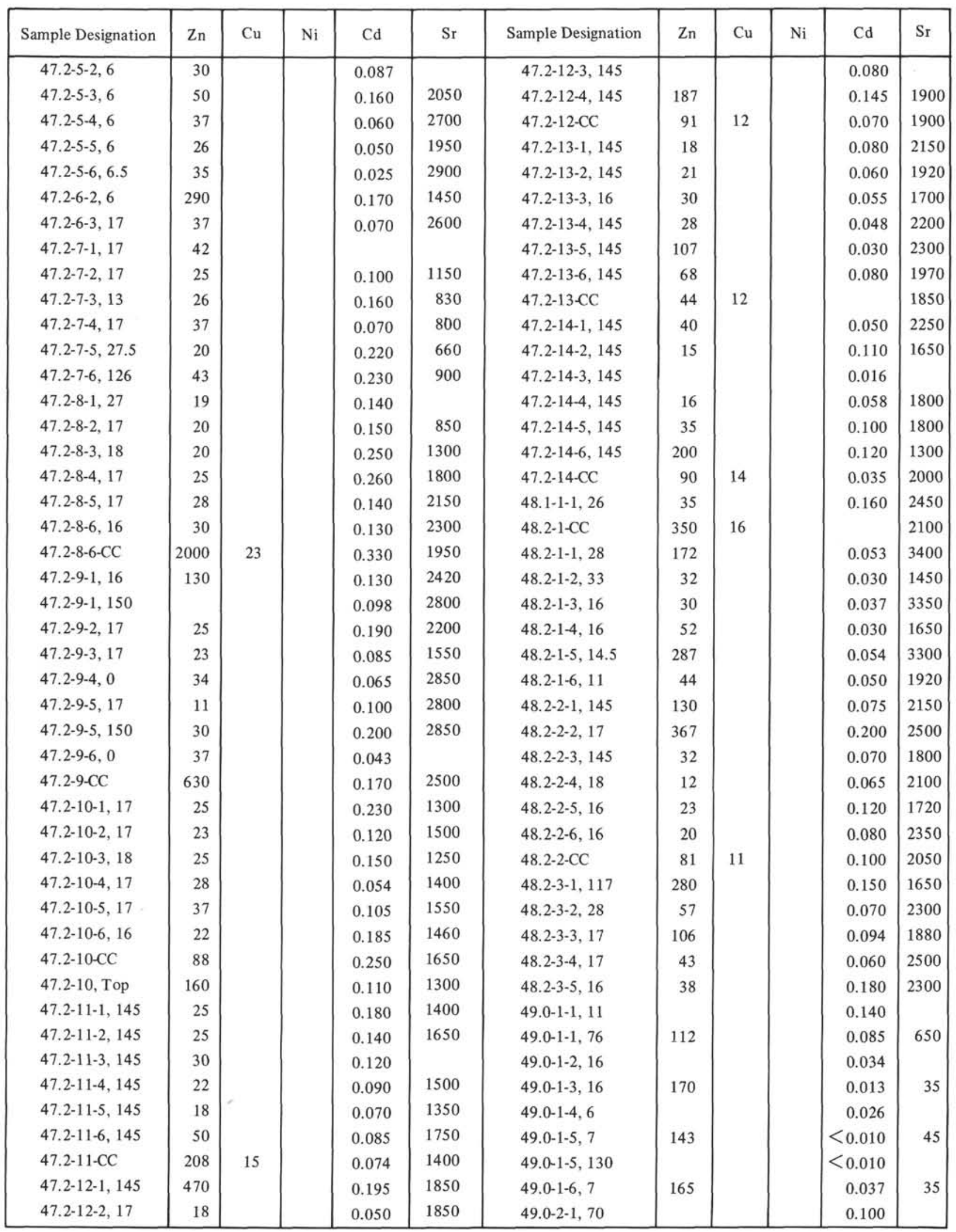


TABLE 15 - Continued

\begin{tabular}{|c|c|c|c|c|c|c|c|c|c|c|c|}
\hline Sample Designation & $\mathrm{Zn}_{\mathrm{n}}$ & $\mathrm{Cu}$ & $\mathrm{Ni}$ & $\mathrm{Cd}$ & $\mathrm{Sr}$ & Sample Designation & $\mathrm{Zn}$ & $\mathrm{Cu}$ & $\mathrm{Ni}$ & $\mathrm{Cd}$ & $\mathrm{Sr}$ \\
\hline $49.1-1-1,77$ & & & & 0.010 & & $51.1-1-6,16$ & & & & 0.012 & \\
\hline $49.1-1-2,16$ & 144 & & & 0.019 & 40 & $51.1-2-1,127$ & 167 & & & & \\
\hline $49.1-1-2,124$ & & & & 0.050 & & $51.1-2-2,8$ & & & & 0.042 & \\
\hline $49.1-1-3,22$ & 198 & & & 0.049 & & $52.0-1-1,27$ & 92 & & & 0.025 & 25 \\
\hline $49.1-1-4,117$ & 84 & & & 0.130 & 350 & $52.0-1-2,8$ & & & & 0.270 & \\
\hline $49.1-1-5,38$ & & & & 0.028 & & $52.0-1-2,30$ & 330 & 130 & & 0.054 & 90 \\
\hline 49.1-1-5, & 280 & 44 & & 0.038 & 120 & $52.0-1-3,8$ & 127 & & & 0.068 & 10 \\
\hline $49.1-2-\mathrm{CC}$ & 30 & & & 0.010 & 35 & $52.0-1-4,8$ & 20 & & & 0.054 & \\
\hline $49.1-2-1,145$ & 94 & & & 0.060 & 1120 & $52.0-1-5,10$ & 107 & & & 0.012 & 75 \\
\hline $49.1-2-2,145$ & & & & 0.043 & & $52.0-1-6,9$ & & & & 0.018 & \\
\hline $49.1-2-2,140$ & & & & & & $52.0-2-\mathrm{CC}$ & 330 & 165 & 80 & $<0.010$ & 50 \\
\hline $49.1-2-3,145$ & 1050 & & & & 1360 & $52.0-2-2,7$ & 98 & & & $<0.010$ & 40 \\
\hline $50.0-2-1,145$ & 1600 & & & 0.105 & 650 & $52.0-2-3,7$ & & & & 0.028 & \\
\hline $50.0-2-2,150$ & 1650 & & & 0.160 & 950 & $52.0-2-4,8$ & 190 & & & 0.012 & 60 \\
\hline $50.0-2-3,150$ & 365 & 165 & & 0.060 & 120 & $52.0-2-5,18$ & & & & 0.011 & \\
\hline $50.0-2-4,150$ & 188 & & & 0.050 & 800 & $52.0-2-6,145$ & 440 & 136 & 80 & 0.051 & 80 \\
\hline $50.0-2-6,150$ & 685 & & & 0.071 & 400 & $52.0-3-1,16$ & & & & 0.010 & \\
\hline $50.1-1-1,62$ & & & & 0.036 & & $52.0-3-2,19$ & 113 & & & 0.013 & 15 \\
\hline $50.1-1-2,15$ & & & & $<0.010$ & & $52.0-3-3,16$ & & & & 0.010 & \\
\hline $50.1-1-3,7$ & 100 & & & 0.030 & 250 & $52.0-3-4,16$ & 182 & & & 0.010 & 15 \\
\hline $50.1-1-4,17$ & & & & 0.021 & & $52.0-3-5,7$ & & & & $<0.010$ & \\
\hline $50.1-1-5,15$ & 90 & & & 0.045 & 650 & $52.0-3-6,6$ & 100 & & & $<0.010$ & 35 \\
\hline $50.1-1-6,16$ & & & & 0.076 & & $52.0-4-2,7$ & & & & $<0.010$ & \\
\hline $50.1-2-1,130$ & 75 & & & 0.029 & 200 & $52.0-4-3,5$ & 103 & & & 0.016 & 650 \\
\hline $50.1-2-3,4$ & & & & 0.045 & 200 & $52.0-4-5,6$ & 107 & & & $<0.010$ & 55 \\
\hline $50.1-2-3,35$ & 156 & & & $\begin{array}{l}0.043 \\
0.031\end{array}$ & & $52.0-4-6,128$ & 134 & & & $<0.010$ & \\
\hline $50.1-2-4,12$ & & & & 0.031 & & $52.0-5-2,14$ & 150 & & & $<0.010$ & 30 \\
\hline & & & & 0.053 & & $52.0-5-3,15$ & 294 & & & $<0.010$ & 300 \\
\hline $50.1-2-4,5$ & 250 & 246 & & 0.048 & $<20$ & $52.0-5-3,18$ & 294 & & & & \\
\hline $50.1-2-5,8$ & 155 & & & 0.011 & 300 & $52.0-5-4,18$ & 142 & & & $<0.010$ & 30 \\
\hline $50.1-3-1,145$ & 206 & 210 & 220 & 0.112 & $<20$ & $52.0-5-5,18$ & & & & 0.018 & \\
\hline $50.1-3-3,15$ & 165 & & & 0.023 & 55 & $52.0-6-\mathrm{CC}$ & 177 & 310 & & 0.040 & 45 \\
\hline $50.1-3-4,16$ & & & & 0.072 & & $52.0-6-1,24$ & 150 & & & & \\
\hline $50.1-3-5,28$ & 143 & & & 0.043 & 35 & $52.0-7-\mathrm{CC}$ & 120 & 66 & 57 & $<0.010$ & 60 \\
\hline $51.0-1-\mathrm{CC}$ & & & & 0.280 & & $52.0-7-2,37$ & & & & 0.025 & \\
\hline $51.0-1-1,11$ & 310 & & & 0.140 & $<10$ & $52.0-8-1,145$ & 160 & 220 & 205 & 0.024 & 65 \\
\hline $51.0-1-3,6$ & 225 & & & 0.080 & $<10$ & $52.0-8-2,145$ & 213 & 200 & & 0.028 & 35 \\
\hline $51.0-1-4,6$ & 204 & & & 0.310 & & $52.0-8-3,21$ & 140 & & & 0.010 & 55 \\
\hline $51.0-1-5,15$ & 204 & & & 0.014 & & $52.0-8-3,40$ & 213 & 200 & 220 & 0.045 & \\
\hline & & & & 0.014 & & $52.0-8-4,145$ & 117 & & & 0.010 & 40 \\
\hline $51.0-1-6,16$ & & & & 0.040 & & $52.0-8-5,145$ & 254 & 328 & & $<0.010$ & 55 \\
\hline $51.0-2-\mathrm{CC}$ & 2600 & 168 & 245 & 0.480 & $<20$ & $52.0-8-6,145$ & 186 & & & 0.022 & \\
\hline $51.1-1-145$ & 320 & & & $<0.01$ & & $52.0-9-\mathrm{CC}$ & 148 & 170 & 340 & 0.026 & 70 \\
\hline $51.1-1-1,15$ & 115 & & & 0.020 & 20 & $52.0-9-\mathrm{CC}$ & 148 & 170 & & 0.026 & \\
\hline $51.1-1-2,16$ & & & & $<0.010$ & & $52.0-10-\mathrm{CC}$ & 605 & 204 & & 0.060 & 15 \\
\hline $51.1-1-3,30$ & 108 & & & 0.015 & 35 & $53.0-1-1,10$ & & & & 0.050 & \\
\hline $51.1-1-4,16$ & & & & 0.040 & & $53.0-1-2,144$ & 86 & & & 0.012 & 60 \\
\hline $51.1-1-4,30$ & 240 & 80 & 85 & 0.026 & $<20$ & $53.0-1-3,17$ & & & & 0.011 & \\
\hline $51.1-1-5,16$ & 82 & & & 0.020 & 25 & $53.0-2-\mathrm{CC}$ & & & & 0.120 & \\
\hline
\end{tabular}


TABLE 15 - Continued

\begin{tabular}{|c|c|c|c|c|c|c|c|c|c|c|c|}
\hline Sample Designation & $\mathrm{Zn}$ & $\mathrm{Cu}$ & $\mathrm{Ni}$ & $\mathrm{Cd}$ & $\mathrm{Sr}$ & Sample Designation & $\mathrm{Zn}$ & $\mathrm{Cu}$ & $\mathrm{Ni}$ & $\mathrm{Cd}$ & $\mathrm{Sr}$ \\
\hline $53.0-2-\mathrm{CC}$ & 370 & & & 0.043 & & $54.0-6-5,17$ & & & & 0.018 & \\
\hline $53.0-3-1,12$ & 92 & & & $<0.010$ & 65 & $54.0-7-1,138$ & 280 & & & 0.050 & 1050 \\
\hline $53.0-4-2,0$ & 200 & 38 & 40 & $<0.010$ & & $54.0-7-2,34$ & & & & 0.015 & \\
\hline $53.0-6-2,65$ & 23 & & & 0.350 & 25 & $54.0-7-3,16$ & 98 & & & 0.200 & 65 \\
\hline $53.0-6-2,66$ & & & & 0.260 & & $54.0-9-1$ & 40 & & & 0.400 & 200 \\
\hline $53.0-6-\mathrm{CC}$ & 152 & 116 & & 0.160 & 15 & $55.0-1-2,7$ & 52 & & & 0.140 & 1200 \\
\hline $53.0-7-1,150$ & & & & 0.120 & & $55.0-1-2,30$ & 150 & & & 0.089 & 2850 \\
\hline $53.0-8-\mathrm{CC}$ & 60 & & 140 & $<0.010$ & & $55.0-1-3,7$ & & & & 0.160 & \\
\hline $53.1-1-2,7$ & 130 & & & $<0.010$ & 50 & $55.0-1-4,7$ & 28 & & & 0.120 & 1850 \\
\hline $53.1-1-3,2$ & 220 & 162 & 40 & 0.020 & & $55.0-1-5,7$ & & & & 0.150 & \\
\hline $53.1-1-3,18$ & 115 & & & 0.014 & & $55.0-1-6,7$ & 23 & & & 0.270 & 2600 \\
\hline $53.1-1-4,17$ & & & & 0.010 & & $55.0-2-1,7$ & 19 & & & 0.076 & 1250 \\
\hline $53.1-1-5,18$ & 88 & & & 0.010 & 150 & $55.0-2-2,7$ & 18 & & & 0.170 & 2850 \\
\hline $53.1-2-1,145$ & 730 & & & 0.085 & 100 & $55.0-2-3,16$ & 500 & & & 0.037 & 200 \\
\hline $53.1-2-2,145$ & 300 & & & 0.031 & 80 & $55.0-2-3-\mathrm{CC}$ & 400 & & & 0.018 & 55 \\
\hline $53.1-2-3,16$ & & & & 0.018 & & $55.0-2-4,15$ & 12 & & & 0.140 & 3100 \\
\hline $53.1-2-3,90$ & 86 & & & $<0.010$ & 148 & $55.0-2-5,7$ & & & & 0.120 & \\
\hline $53.1-2-4,10$ & & & & 0.026 & & $55.0-2-5,70$ & 15 & & & 0.130 & 3200 \\
\hline $53.1-2-5,7$ & 125 & & & $<0.010$ & 100 & $55.0-2-6,32$ & & & & 0.065 & \\
\hline $53.1-2-6,7$ & & & & 0.030 & & $55.0-3$-Top of core & & & & 0.052 & \\
\hline $53.1-3-1,150$ & 200 & 163 & 42 & 0.018 & 95 & $55.0-3-1,16$ & 20 & & & 0.010 & 3250 \\
\hline $53.1-3-2,16$ & 182 & & & $<0.010$ & 90 & $55.0-3-3,14$ & 18 & & & 0.070 & 900 \\
\hline $53.1-3-2,40$ & 220 & 162 & 40 & 0.020 & 130 & $55.0-3-4,145$ & 16 & 16 & & 0.095 & 2800 \\
\hline $53.1-3-3,23.5$ & & & & 0.017 & & $55.0-3-5,15$ & 18 & & & 0.072 & 2000 \\
\hline $53.1-3-4,26$ & 90 & & & $<0.010$ & & $55.0-4-1,13$ & 14 & & & 0.080 & 2300 \\
\hline $53.1-3-5,23$ & & & & 0.014 & & $55.0-4-2,145$ & 24 & & & 0.037 & 2000 \\
\hline $53.2-1-1,21$ & & & & 0.011 & & $55.0-4-3,7$ & 15 & & & 0.066 & 2250 \\
\hline $53.2-1-2,16$ & 90 & & & 0.010 & 100 & $55.0-4-4,145$ & 29 & & & 0.032 & 70 \\
\hline $53.2-1-3,22$ & & & & $<0.010$ & & $55.0-4-5,7$ & 25 & & & 0.023 & 2150 \\
\hline $53.2-1-4,7$ & 90 & & & & 30 & $55.0-4-6,145$ & & & & 0.031 & \\
\hline $53.2-1-5,7$ & & & & 0.010 & & $55.0-5-1,7$ & 14 & & & 0.043 & 2650 \\
\hline $53.2-1-6,7$ & 97 & & & 0.012 & 100 & $55.0-5-3,8$ & & & & 0.028 & \\
\hline $53.2-4-\mathrm{CC}$ & 92 & & & 0.011 & 185 & $55.0-5-5,7$ & 15 & & & 0.044 & 2750 \\
\hline $53.2-5-\mathrm{CC}$ & 156 & & & 0.023 & 130 & $55.0-6-1,7$ & & & & 0.033 & \\
\hline $54.0-1-1,5$ & 124 & & & 0.050 & 800 & $55.0-6-3,17$ & & & & 0.070 & \\
\hline $54.0-1-1,74$ & & & & $<0.010$ & & $55.0-6-5,16$ & 24 & & & 0.010 & 1550 \\
\hline $54.0-1-2,7$ & 278 & & & $<0.010$ & 180 & $55.0-7-1,14$ & 18 & & & 0.070 & 2000 \\
\hline $54.0-1-2,20$ & 100 & & & 0.012 & 140 & $55.0-7-3,18$ & 14 & & & 0.017 & 1400 \\
\hline $54.0-1-3,35$ & & & & $<0.010$ & 140 & $55.0-7-5,19$ & 15 & & & 0.014 & 2200 \\
\hline $54.0-2-1,17$ & 75 & & & & & $55.0-8-1,15$ & & & & 0.038 & \\
\hline $54.0-2-2,23$ & & & & & & $55.0-8-3,16$ & 18 & & & 0.076 & 2150 \\
\hline & & & & 0.017 & & $55.0-8-5,18$ & 30 & & & & 6600 \\
\hline $54.0-2-4,16$ & 72 & & & 0.011 & & $55.0-9$ & 84 & 28 & & 0.084 & \\
\hline $54.0-3-1,140$ & 148 & 40 & & 0.038 & 350 & $55.0-10-2,10$ & 15 & & & 0.074 & 1950 \\
\hline $54.0-4-1,108$ & & & & 0.012 & & $55.0-11-5,16$ & 19 & & & 0.080 & 3400 \\
\hline $54.0-4-2,14$ & 148 & & & 0.026 & 20 & $55.0-11-3,17$ & 30 & & & 0.038 & 2500 \\
\hline $54.0-6-1,145$ & 108 & 42 & & 0.028 & 140 & $55.0-11-5,7$ & 20 & & & 0.010 & 1550 \\
\hline $54.0-6-2,16$ & & & & 0.010 & & $55.0-12-1,13$ & 18 & & & 0.060 & 2000 \\
\hline $54.0-6-3,23$ & 65 & & & 0.020 & 150 & $55,0-12-2,13$ & 25 & & & 0.052 & 1100 \\
\hline $54.0-6-4,16$ & & & & 0.018 & & $55.0-12-3,8$ & 12 & & & 0.090 & 2650 \\
\hline $54.0-6-4,78$ & 77 & & & 0.014 & & $55.0-12-5,7$ & 52 & & & 0.026 & 5600 \\
\hline
\end{tabular}


TABLE 15 - Continued

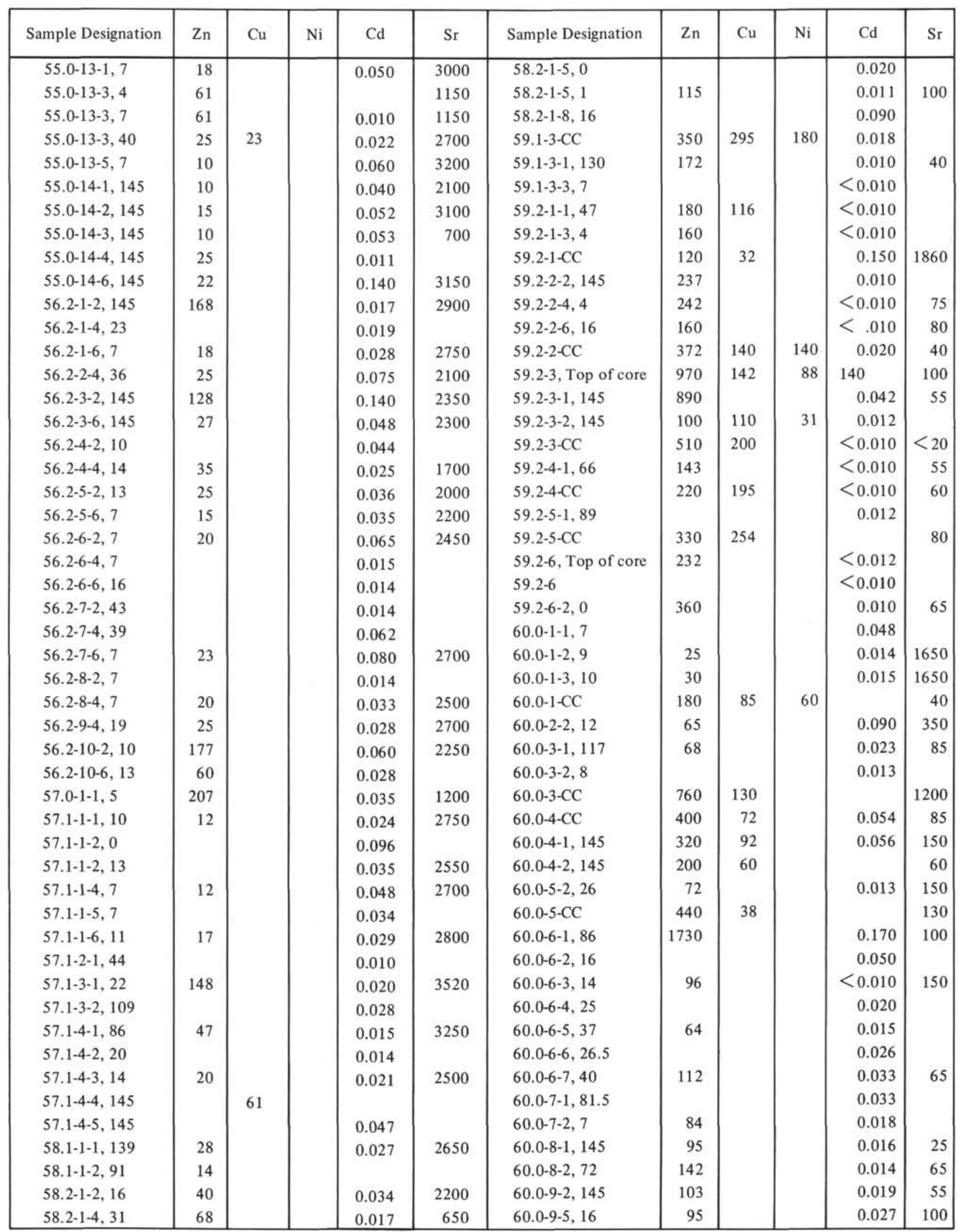


TABLE 16

Neutron Activation Analysis of Cherts

\begin{tabular}{|l|c|c|c|c|}
\hline \multirow{2}{*}{ Sample No. } & \multicolumn{4}{|c|}{ Radioisotope } \\
\cline { 2 - 5 } & $\mathrm{Mn}^{56}$ & $\mathrm{Sc}^{46}$ & $\mathrm{~W}^{187}$ & $\mathrm{Fe}^{59}$ \\
\hline $44.0-3-\mathrm{CC}$ & $(1.9 \pm 0.2) \times 10^{-2}$ & $(7.7 \pm 0.8) \times 10^{-5}$ & $(4.7 \pm 0.5) \times 10^{-2}$ & $(4.7 \pm 0.5) \times 10^{-2}$ \\
$44.0-3-\mathrm{CC}$ & $(1.1 \pm 0.1) \times 10^{-2}$ & $(7.5 \pm 0.7) \times 10^{-5}$ & $(5.4 \pm 0.7) \times 10^{-2}$ & $(5.4 \pm 0.5) \times 10^{-1}$ \\
$46.0-1-6$ & $(1.1 \pm 0.1) \times 10^{-2}$ & $(4.2 \pm 0.4) \times 10^{-4}$ & $(1.5 \pm 0.2) \times 10^{-2}$ & $(5.2 \pm 0.5) \times 10^{-1}$ \\
$46.0-1-6$ & $(5.3 \pm 0.5) \times 10^{-3}$ & $(1.6 \pm 0.2) \times 10^{-4}$ & $(1.1 \pm 0.1) \times 10^{-1}$ & $(2.4 \pm 0.2) \times 10^{-1}$ \\
$46.0-1-6$ & $(1.1 \pm 0.1) \times 10^{-1}$ & $(1.3 \pm 0.1) \times 10^{-3}$ & $(3.5 \pm 0.4) \times 10^{-1}$ & $4.3 \pm 0.4$ \\
$47.2-12-2$ & $(4.5 \pm 0.4) \times 10^{-3}$ & $(1.5 \pm 0.1) \times 10^{-4}$ & $(7.0 \pm 0.7) \times 10^{-2}$ & $(1.7 \pm 0.2) \times 10^{-1}$ \\
$49.1-2-\mathrm{CC}$ & $(6.4 \pm 0.2) \times 10^{-4}$ & $(1.7 \pm 0.2) \times 10^{-4}$ & $(3.6 \pm 0.4) \times 10^{-2}$ & $(2.4 \pm 0.3) \times 10^{-1}$ \\
$50.0-2-\mathrm{CC}$ & $(2.0 \pm 0.6) \times 10^{-3}$ & $(2.0 \pm 0.2) \times 10^{-4}$ & $(8.4 \pm 0.8) \times 10^{-3}$ & $(2.0 \pm 0.2) \times 10^{-1}$ \\
$50.0-2-\mathrm{CC}$ & $(6.2 \pm 0.6) \times 10^{-2}$ & $(6.1 \pm 0.6) \times 10^{-4}$ & - & $2.4 \pm 0.2$ \\
$50.0-2-\mathrm{CC}$ & $(1.9 \pm 0.2) \times 10^{-2}$ & $(1.7 \pm 0.2) \times 10^{-4}$ & $(8.8 \pm 0.8) \times 10^{-2}$ & $(4.4 \pm 0.5) \times 10^{-1}$ \\
$50.0-2-\mathrm{CC}$ & $(2.3 \pm 0.2) \times 10^{-3}$ & $(2.3 \pm 0.2) \times 10^{-4}$ & $(9.8 \pm 0.9) \times 10^{-2}$ & $(6.0 \pm 0.6) \times 10^{-1}$ \\
$59.2-6$ & $(1.4 \pm 0.1) \times 10^{-1}$ & $(1.4 \pm 0.1) \times 10^{-3}$ & - & $2.3 \pm 0.2$ \\
$59.2-6, \mathrm{TOP}$ & $(4.5 \pm 0.4) \times 10^{-2}$ & $(6.2 \pm 0.6) \times 10^{-4}$ & - & $2.6 \pm 0.3$ \\
$59.2-6$ TOP & $(7.9 \pm 0.8) \times 10^{-2}$ & $(3.1 \pm 0.3) \times 10^{-3}$ & $(3.9 \pm 0.4) \times 10^{-1}$ & $10.1 \pm 1.0$ \\
\hline
\end{tabular}


TABLE 17

Chemical Analyses of Recent Sediments

TABLE 17A-Abyssal Red Clay

\begin{tabular}{|c|c|c|c|c|c|c|}
\hline Element & 1 & 2 & 3 & 4 & 5 & Description \\
\hline $\mathrm{SiO}_{2}$ & 54.48 & 54.28 & 55.16 & 50.77 & 52.45 & \multirow{12}{*}{$\begin{array}{l}\text { 1. Average } 51 \text { samples red clays Pacific, } \\
\text { Atlantic and Indian oceans (Clarke, } \\
\text { Steiger) } \\
\text { 2. Average data of Challenger Expedition } \\
\text { 3. Average data of Meteor Expedition } \\
\text { 4. Average } 6 \text { samples red clay S-W part of } \\
\text { North Pacific Ocean (Ishibashi, Huroda) } \\
\text { 5. Average } 16 \text { samples S-W part of North } \\
\text { Pacific Ocean (Hamoguchi) }\end{array}$} \\
\hline $\mathrm{TiO}_{2}$ & 0.98 & - & 0.56 & - & 0.84 & \\
\hline $\mathrm{Al}_{2} \mathrm{O}_{3}$ & 15.94 & 16.41 & 16.79 & 20.75 & 16.05 & \\
\hline $\mathrm{Fe}_{2} \mathrm{O}_{3}$ & 8.66 & 13.58 & 5.65 & 10.54 & 8.26 & \\
\hline $\mathrm{Fe} 0$ & 0.84 & 1.26 & 1.38 & - & - & \\
\hline Mno & 1.21 & 1.62 & 4.13 & 2.01 & 1.12 & \\
\hline $\mathrm{Mg} 0$ & 3.31 & 1.76 & 2.76 & 3.08 & 3.35 & \\
\hline $\mathrm{Ca} 0$ & 1.96 & 0.74 & 0.27 & 1.72 & 2.44 & \\
\hline $\mathrm{Na}_{2} \mathrm{O}$ & 2.05 & 1.37 & 2.41 & 1.39 & 4.98 & \\
\hline $\mathrm{K}_{2} \mathrm{O}$ & 2.85 & 1.61 & 1.09 & 2.78 & 2.57 & \\
\hline $\mathrm{H}_{2} \mathrm{O}$ & 7.04 & 7.02 & 9.08 & 9.75 & 7.34 & \\
\hline $\mathrm{P}_{2} \mathrm{O}_{5}$ & 0.30 & 0.35 & 0.32 & 0.28 & 0.34 & \\
\hline
\end{tabular}

TABLE 17B-Radiolarian Ooze

\begin{tabular}{|l|c|l|}
\hline Element & Composition (\%) & Description \\
\hline $\mathrm{SiO}_{2}$ & 56.86 & From southwest part \\
$\mathrm{Al}_{2} \mathrm{O}_{3}$ & 22.28 & of North Pacific \\
$\mathrm{Fe}_{2} \mathrm{O}_{3}$ & 7.50 & \\
$\mathrm{Ca} 0$ & 1.85 & \\
$\mathrm{Mg} 0$ & 3.34 & \\
$\mathrm{Na}_{2} \mathrm{O}$ & 1.08 & \\
$\mathrm{~K}_{2} \mathrm{O}$ & 0.39 & \\
\hline
\end{tabular}

TABLE 17C-Diatom Ooze

\begin{tabular}{|l|r|r|}
\hline Compound & Composition (\%) & Description \\
\hline $\mathrm{Si}_{2}$ & 69.72 & Murray and \\
$\mathrm{Al}_{2} \mathrm{O}_{3}$ & 0.55 & Renard \\
$\mathrm{Fe}_{2} \mathrm{O}_{3}$ & 0.39 & \\
$\mathrm{CaCO}_{3}$ & 19.29 & \\
$\mathrm{CaSO}_{4}$ & 0.29 & \\
$\mathrm{Ca}_{3} \mathrm{P}_{2} \mathrm{O}_{8}$ & 0.41 & \\
$\mathrm{MgCO}_{3}$ & 1.13 & \\
Insoluble Residue & 4.47 & \\
Loss on ignition & 5.30 & \\
\hline
\end{tabular}


TABLE 17 - Continued

TABLE 17D-Globigerina Ooze (non-carbonate part) and Red Clay

\begin{tabular}{|l|r|r|r|c|}
\hline \multicolumn{3}{|c|}{ Sample No. $^{\mathrm{a}}$} & $\begin{array}{c}\text { Average } \\
\text { Composition } \\
\text { of Red Clay }\end{array}$ \\
\hline Compound & 1602 & 1562 & 1572 & 56.70 \\
$\mathrm{SiO}_{2}$ & 56.76 & 55.84 & 51.10 & 0.91 \\
$\mathrm{TiO}_{2}$ & 0.65 & 1.49 & 1.41 & 17.35 \\
$\mathrm{Al}_{2} 0_{3}$ & 15.52 & 17.89 & 18.73 & 8.93 \\
$\mathrm{Fe}_{2} \mathrm{O}_{3}$ & 9.03 & 11.18 & 11.11 & 1.21 \\
$\mathrm{Mn0}$ & 0.59 & 1.42 & 1.18 & 3.62 \\
$\mathrm{Mg} 0$ & 4.53 & 1.38 & 5.64 & 2.64 \\
$\mathrm{Ca0}$ & 4.76 & 1.20 & 1.69 & 5.38 \\
$\mathrm{Na}_{2} \mathrm{O}$ & 7.50 & 4.62 & 4.63 & 2.78 \\
$\mathrm{~K}_{2} \mathrm{O}$ & 2.13 & 4.22 & 3.33 & 0.48 \\
$\mathrm{P}_{2} \mathrm{O}_{5}$ & 0.54 & 0.75 & 1.18 & \\
$\mathrm{Total}_{\mathrm{CaCO}}+$ & & & & \\
$\mathrm{MgCO}_{3}$ & 56.59 & 68.75 & 80.23 & \\
\hline
\end{tabular}

${ }^{\mathrm{a}}$ Hamoguchi's sample numbers 
TABLE 18

Chemical Composition of Basalts and Volcanic Ash from Leg 6

\begin{tabular}{|c|c|c|c|c|c|c|c|c|}
\hline & \multicolumn{4}{|c|}{ Leg 6 Samples } & \multicolumn{4}{|c|}{ Tholeitic Basalts (Engel, Engel, 1968) } \\
\hline & $\begin{array}{c}\text { Site } 57 \\
\text { Oligocene } \\
\text { Diabasic } \\
\text { Basalt }\end{array}$ & $\begin{array}{c}\text { Site } 53 \\
\text { Oligocene- } \\
\text { Miocene } \\
\text { Basalt }\end{array}$ & Site 54 & $\begin{array}{c}\text { 45.1-3-CC } \\
\text { Lithified } \\
\text { Ash }\end{array}$ & $\begin{array}{l}\text { Atlantic } \\
\text { Ocean }\end{array}$ & $\begin{array}{l}\text { Pacific } \\
\text { Ocean }\end{array}$ & $\begin{array}{l}\text { Indian } \\
\text { Ocean }\end{array}$ & Average \\
\hline $\mathrm{SiO}_{2}$ & 49.60 & 51.30 & 18.600 & 91.00 & 49.80 & 50.25 & 50.29 & 50.11 \\
\hline $\mathrm{TiO}_{2}$ & 2.63 & 0.11 & 0.470 & 0.26 & 1.33 & 1.56 & 1.21 & 1.37 \\
\hline $\mathrm{Al}_{2} \mathrm{O}_{3}$ & 15.70 & 16.60 & 7.850 & 4.47 & 16.87 & 16.09 & 17.16 & 16.71 \\
\hline $\mathrm{F}_{2} \mathrm{O}$ & & & & & 7.28 & 7.20 & 6.51 & 7.00 \\
\hline $\mathrm{Fe}_{2} \mathrm{O}_{3}$ & 12.50 & 9.10 & 4.740 & 2.14 & 2.07 & 2.72 & 2.26 & 2.35 \\
\hline $\mathrm{Mn} 0$ & 0.18 & 0.07 & 0.102 & 0.12 & 0.16 & 0.19 & 0.16 & 0.17 \\
\hline $\mathrm{Mg0}$ & 5.69 & 6.68 & 3.550 & 1.10 & 8.01 & 7.02 & 7.75 & 7.59 \\
\hline $\mathrm{CaO}$ & 10.90 & 5.00 & 30.900 & $<0.88$ & 11.38 & 11.81 & 11.55 & 11.58 \\
\hline $\mathrm{Na}_{2} \mathrm{O}$ & - & - & - & - & 2.78 & 2.81 & 2.83 & 2.81 \\
\hline $\mathrm{KaO}$ & - & - & - & - & 0.18 & 0.20 & 0.19 & 0.19 \\
\hline $\mathrm{P}_{2} \mathrm{O}_{5}$ & - & - & - & - & 0.14 & 0.15 & 0.09 & 0.13 \\
\hline
\end{tabular}


TABLE 19

Results of the Analysis of Fluorine

\begin{tabular}{|c|c|c|c|c|}
\hline Hole & Core & Section & Sampled at & Fluorine\% (dry material) \\
\hline 51.1 & 1 & 3 & $30-32$ & 0.101 \\
51.1 & 1 & 5 & $16-18$ & 0.080 \\
51.1 & 2 & 1 & $127-129$ & 0.122 \\
51.1 & 2 & 2 & 145 & 0.160 \\
55.0 & 1 & 2 & $7-9$ & 0.074 \\
55.0 & 3 & 4 & $145-150$ & 0.050 \\
55.0 & 6 & 3 & $17-19$ & 0.030 \\
55.0 & 7 & 5 & $19-21$ & 0.045 \\
55.0 & 11 & 3 & $17-19$ & 0.025 \\
55.0 & 13 & 1 & $7-9$ & 0.020 \\
55.0 & 14 & 4 & $145-150$ & 0.025 \\
55.0 & 14 & 6 & $145-150$ & 0.020 \\
\hline
\end{tabular}

Analysis made by V.S. Bykova 